Supporting Information for:

\title{
Palladium-Catalyzed Hydrocarbonylative Cyclization of 1,5-Dienes
}

Suchen Zou, ${ }^{\dagger}$ Bao Gao, ${ }^{\dagger}$ Yao Huang, ${ }^{\dagger}$ Tianze Zhang, ${ }^{\dagger}$ and Hanmin Huang* ${ }^{\dagger}, \ddagger$

${ }^{\dagger}$ Hefei National Laboratory for Physical Sciences at the Microscale and Department of Chemistry, Center for Excellence in Molecular Synthesis, University of Science and Technology of China, Chinese Academy of Sciences, Hefei, 230026, P. R. China

${ }^{\star}$ State Key Laboratory of Applied Organic Chemistry, Lanzhou University, Lanzhou, 730000, P. R. China

*E-mail: hanmin@ustc.edu.cn

\section{CONTENTS}

1 General experimental details and materials

2 Preparation and spectral data of 1,5-dienes

3 Optimization of the reaction conditions

4 General procedure for the catalytic reaction and spectral data of products

5 Gram-scale synthesis of $2 a$

6 Mechanistic studies

7 References

8 Copies of ${ }^{1} \mathrm{H}$ NMR, ${ }^{19} \mathrm{~F}$ NMR and ${ }^{13} \mathrm{C}$ NMR of materials and products 


\section{General experiment details and materials}

Experimental: All non-aqueous reactions and manipulations were using standard Schlenk techniques. All solvents before use were dried and degassed by standard methods and stored under nitrogen atmosphere. All reactions were monitored by TLC with silica gel-coated plates. NMR spectra were recorded on BRUKER Avence III 400 $\mathrm{MHz}$ spectrometers. Chemical shifts were reported in parts per million (ppm) down field from TMS with the solvent resonance as the internal standard. Coupling constants $(J)$ were reported in $\mathrm{Hz}$ and refered to apparent peak multiplications. High resolution mass spectra (HRMS) were recorded on Bruker MicroTOF-QII mass (ESI). GC analysis were performed on Agilent 7890B with Hp-5 column. GC-MS analysis were performed with Agilent 7890B/5975B GC-MS system. Dienes were synthesized according to the reported methods. ${ }^{1}$

\section{Preparation and spectral data of 1,5-dienes}

Method A: Synthesis of substrate $1 a^{1 \mathrm{a}}$

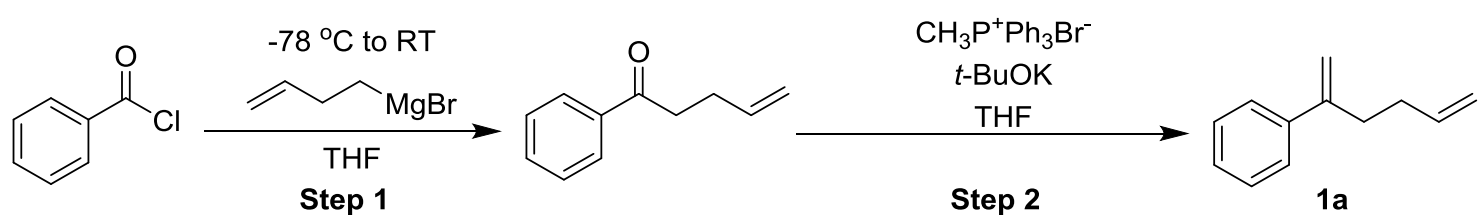

Step 1: To a mixture of the benzoyl chloride (4.6g, $33 \mathrm{mmol}, 1.1$ equiv) and $\mathrm{CuI}(279$ $\mathrm{mg}, \quad 1.5 \mathrm{mmol}, 5 \mathrm{~mol} \%)$ in THF $(45 \mathrm{~mL})$ was added $0.5 \mathrm{M}$ solution of 3-butenylmagnesium bromide in THF (60 mL, $30 \mathrm{mmol}, 1.0$ equiv.) at $-78{ }^{\circ} \mathrm{C}$ dropwise. The reaction mixture was stirred at $-78{ }^{\circ} \mathrm{C}$ for 1 hour and at room temperature overnight, before it was quenched by adding sat. aq. $\mathrm{NH}_{4} \mathrm{Cl}$ solution. Then the aqueous solution was extracted with diethyl ether three times. The combined organic phases were dried over $\mathrm{MgSO}_{4}$, filtered and removed in vacuo. The residue was purified by column chromatography on silica gel and eluted with petroleum ether/ethyl acetate (50/1) to afford 1-phenylpent-4-en-1-one (4.5 g, 94\% yield). 
Step 2: To a solution of methyltriphenylphosphonium bromide (25 mmol, 1.25 equiv.) in THF $(80 \mathrm{~mL})$ was added $t$-BuOK $(24 \mathrm{mmol}, 1.2$ equiv. $)$ at $0{ }^{\circ} \mathrm{C}$ and the resulting mixture was stirred for 30 minutes at $0{ }^{\circ} \mathrm{C}$. Then the solution of 1-phenylpent-4-en-1-one (20 mmol, 1.0 equiv.) in THF (20 mL) was added to the reaction mixture dropwise at $0{ }^{\circ} \mathrm{C}$. After 30 minutes, the ice bath was removed and the mixture was stirred overnight at room temperature, before it was quenched by adding sat. aq. $\mathrm{NH}_{4} \mathrm{Cl}$ solution. Then the aqueous solution was extracted with diethyl ether three times. The combined organic phases were dried over $\mathrm{MgSO}_{4}$, filtered and removed in vacuo. The residue was purified by column chromatography on silica gel and eluted with petroleum ether/ethyl acetate (100/1-50/1) to afford hexa-1,5-dien-2-ylbenzene $1 \mathbf{a}(2.6 \mathrm{~g}, 82 \%$ yield $)$.

Other compounds including $\mathbf{1 b} \sim \mathbf{1 n}$, and $\mathbf{1 p}, \mathbf{1 t}$ were prepared by method $\mathbf{A}$.

Hexa-1,5-dien-2-ylbenzene (1a): Known compound. ${ }^{1 \mathrm{a}}$ The title compound was<smiles>C=CCCC(=C)c1ccccc1</smiles>
prepared according to the general procedure (Method A) and purified by column chromatography on silica gel and eluted with petroleum ether/ethyl acetate (100/1) to give the colorless oil (2.6 g, 82\% yield). ${ }^{1} \mathbf{H}$ NMR (400 MHz, $\left.\mathrm{CDCl}_{3}\right) \delta$ 2.19-2.24 (m, 2H), 2.59 (t, J =7.6 Hz, 2H), 4.95-5,07 (m, 3H), $5.28(\mathrm{~s}, 1 \mathrm{H}), 5.79-5.90(\mathrm{~m}, 1 \mathrm{H}), 7.24-7.41(\mathrm{~m}, 5 \mathrm{H}) ;{ }^{13} \mathbf{C} \mathbf{N M R}$ $\left(100 \mathrm{MHz}, \mathrm{CDCl}_{3}\right) \delta 32.6,34.9,112.6,114.9,126.3,127.5,128.4,138.3,141.4,148.1$.

1-(hexa-1,5-dien-2-yl)-4-methylbenzene (1b): Known compound. $^{2 a}$ The title r compound was prepared according to the general procedure (Method A) and purified by column chromatography on silica gel and eluted with petroleum ether/ethyl acetate (100/1) to give the colorless oil (1.63 g, $41 \%$ yield). ${ }^{1} \mathbf{H}$ NMR $\left(400 \mathrm{MHz}, \mathrm{CDCl}_{3}\right) \delta 2.18-2.23$ (m, 2H), $2.33(\mathrm{~s}, 3 \mathrm{H}), 2.57(\mathrm{t}, J=7.6 \mathrm{~Hz}, 2 \mathrm{H}), 4.94-5.02(\mathrm{~m}, 3 \mathrm{H}), 5.25(\mathrm{~d}, J=1.2 \mathrm{~Hz}, 1 \mathrm{H})$, 5.78-5.88 (m, 1H), 7.11-7.13 (d, $J=8.0 \mathrm{~Hz}, 2 \mathrm{H}), 7.28-7.30(\mathrm{~d}, J=8.0 \mathrm{~Hz}, 2 \mathrm{H}) ;{ }^{13} \mathbf{C}$ NMR $\left(100 \mathrm{MHz}, \mathrm{CDCl}_{3}\right) \delta 21.2,32.6,34.9,111.8,114.8,126.1,129.1,137.2,138.4$, 147.8 . 
1-(hexa-1,5-dien-2-yl)-3-methylbenzene (1c): The title compound was prepared according to the general procedure (Method A) and purified by

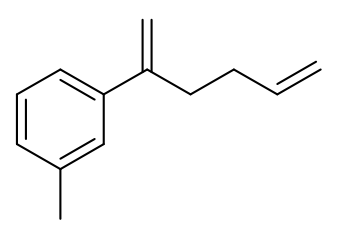
column chromatography on silica gel and eluted with petroleum ether/ethyl acetate (100/1) to give the colorless oil $(1.3 \mathrm{~g}, 36 \%$ yield). ${ }^{1} \mathbf{H}$ NMR $\left(400 \mathrm{MHz}, \mathrm{CDCl}_{3}\right) \delta 2.18(\mathrm{~m}, 2 \mathrm{H}), 2.36(\mathrm{~s}, 3 \mathrm{H})$, $2.58(\mathrm{t}, J=8.0 \mathrm{~Hz}, 2 \mathrm{H}), 4.96-5.05(\mathrm{~m}, 3 \mathrm{H}), 5.26(\mathrm{~s}, 1 \mathrm{H}), 5.80-5.90(\mathrm{~m}, 1 \mathrm{H}), 7.08-7.09$ (m, 1H), 7.19-7.24 (m, 3H); ${ }^{13} \mathbf{C}$ NMR (100 MHz, $\left.\mathrm{CDCl}_{3}\right) \delta 21.7,32.6,34.9,112.4$, 114.8, 123.4, 127.0, 128.2, 128.3, 137.9, 138.4, 141.4, 148.2. HRMS (ESI) calcd. for $\mathrm{C}_{13} \mathrm{H}_{17}[\mathrm{M}+\mathrm{H}]:$ 173.1330, found: 173.1333 .

1-(hexa-1,5-dien-2-yl)-2-methylbenzene (1d): The title compound was prepared<smiles>C=CCCC(=C)c1ccccc1C</smiles>
according to the general procedure (Method A) and purified by column chromatography on silica gel and eluted with petroleum ether/ethyl acetate (100/1) to give the colorless oil $(4.3 \mathrm{~g}, 82 \%$ yield). ${ }^{\mathbf{1}} \mathbf{H}$ NMR $\left(400 \mathrm{MHz}, \mathrm{CDCl}_{3}\right) \delta$ 2.12-2.17 (m, 2H), $2.29(\mathrm{~s}, 3 \mathrm{H}), 2.42$ (t, $J=7.6$ $\mathrm{Hz}, 2 \mathrm{H}), 4.88-5.03(\mathrm{~m}, 3 \mathrm{H}), 5.18(\mathrm{~m}, 1 \mathrm{H}), 5.76-5.86(\mathrm{~m}, 1 \mathrm{H}), 7.05-7.18(\mathrm{~m}, 4 \mathrm{H}) ;{ }^{13} \mathrm{C}$ NMR $\left(100 \mathrm{MHz}, \mathrm{CDCl}_{3}\right) \delta 20.0,32.1,37.2,114.2,114.8,125.5,126.9,128.5,130.2$, 135.0, 138.4, 143.0, 149.5. HRMS (ESI) calcd. for $\mathrm{C}_{13} \mathrm{H}_{17}[\mathrm{M}+\mathrm{H}]$ : 173.1330, found: 173.1329 .

1-ethyl-4-(hexa-1,5-dien-2-yl)benzene (1e): The title compound was prepared r according to the general procedure (Method A) and purified by column chromatography on silica gel and eluted with petroleum ether/ethyl acetate (100/1) to give the colorless oil $\left(1.2 \mathrm{~g}, 33 \%\right.$ yield). ${ }^{1} \mathbf{H}$ NMR $\left(400 \mathrm{MHz}, \mathrm{CDCl}_{3}\right) \delta 1.23(\mathrm{t}, J=7.6 \mathrm{~Hz}, 3 \mathrm{H}), 2.21$ (q, $J=$ $7.6 \mathrm{~Hz}, 2 \mathrm{H}), 2.57$ (t, $J=7.6 \mathrm{~Hz}, 2 \mathrm{H}), 2.64(\mathrm{q}, J=7.6 \mathrm{~Hz}, 2 \mathrm{H}), 4.94-5.02(\mathrm{~m}, 3 \mathrm{H}), 5.26$ $(\mathrm{s}, 1 \mathrm{H}), 5.79-5.89(\mathrm{~m}, 1 \mathrm{H}), 7.15(\mathrm{~d}, J=8.0 \mathrm{~Hz}, 2 \mathrm{H}), 7.32(\mathrm{~d}, J=8.0 \mathrm{~Hz}, 2 \mathrm{H}) ;{ }^{13} \mathrm{C}$ NMR $\left(100 \mathrm{MHz}, \mathrm{CDCl}_{3}\right) \delta 15.7,28.6,32.6,34.8,111.9,114.8,126.2,127.9,138.4$, 138.6, 143.6, 147.8. HRMS (ESI) calcd. for $\mathrm{C}_{14} \mathrm{H}_{19}[\mathrm{M}+\mathrm{H}]$ : 187.1487, found: 187.1474 . 
1-(tert-butyl)-4-(hexa-1,5-dien-2-yl)benzene (1f): The title compound was prepared (-Bu according to the general procedure (Method A) and purified by column chromatography on silica gel and eluted with petroleum ether/ethyl acetate (100/1) to give the colorless oil (2.3 g, 58\% yield). ${ }^{1} \mathbf{H}$ NMR (400 MHz, $\left.\mathrm{CDCl}_{3}\right) \delta 1.32$ (s, 9H), 2.20-2.26 $(\mathrm{m}, 2 \mathrm{H}), 2.56-2.60(\mathrm{~m}, 2 \mathrm{H}), 4.95-5.05(\mathrm{~m}, 3 \mathrm{H}), 5.28(\mathrm{~d}, J=1.6 \mathrm{~Hz}, 1 \mathrm{H}), 5.80-5.90(\mathrm{~m}$, 1H), $7.35(\mathrm{~m}, 4 \mathrm{H}), ;{ }^{13} \mathbf{C} \mathbf{N M R}\left(100 \mathrm{MHz}, \mathrm{CDCl}_{3}\right) \delta 31.5,32.7,34.6,34.7,111.9,114.8$, 125.3, 125.8, 138.3, 138.4, 147.6, 150.5. HRMS (ESI) calcd. for $\mathrm{C}_{16} \mathrm{H}_{23}[\mathrm{M}+\mathrm{H}]$ : 215.1800, found: 215.1801 .

1-(hexa-1,5-dien-2-yl)-4-methoxybenzene (1g): Known compound. $^{2 b}$ The title<smiles>C=CCCC(=C)c1ccc(OC)cc1</smiles>
compound was prepared according to the general procedure (Method A) and purified by column chromatography on silica gel and eluted with petroleum ether/ethyl acetate (50/1) to give the colorless oil (1.7 g, 65\% yield). ${ }^{1} \mathbf{H}$ NMR (400 $\left.\mathrm{MHz}, \mathrm{CDCl}_{3}\right) \delta 2.18-2.23(\mathrm{~m}, 2 \mathrm{H}), 2.56(\mathrm{t}, J=8.0 \mathrm{~Hz}, 2 \mathrm{H}), 3.79(\mathrm{~s}, 3 \mathrm{H}), 4.94-5.03(\mathrm{~m}$, $3 \mathrm{H}), 5.21(\mathrm{~d}, J=1.2 \mathrm{~Hz}, 1 \mathrm{H}), 5.79-5.89(\mathrm{~m}, 1 \mathrm{H}), 6.84-6.87(\mathrm{~m}, 2 \mathrm{H}), 7.32-7.36(\mathrm{~m}$, 2H); ${ }^{13}$ C NMR (100 MHz, $\left.\mathrm{CDCl}_{3}\right) \delta 32.6,34.9,55.3,111.0,113.7,114.8,127.3,133.7$, $138.3,147.3,159.1$.

1-fluoro-4-(hexa-1,5-dien-2-yl)benzene (1h): Known compound. $^{2 b}$ The title<smiles>C=CCCC(=C)c1ccc(F)cc1</smiles>
compound was prepared according to the general procedure (Method A) and purified by column chromatography on silica gel and eluted with petroleum ether/ethyl acetate (100/1) to give the colorless oil $\left(0.9 \mathrm{~g}, 48 \%\right.$ yield). ${ }^{1} \mathbf{H}$ NMR $\left(400 \mathrm{MHz}, \mathrm{CDCl}_{3}\right) \delta 2.16-2.22(\mathrm{~m}$, 2H), $2.56(\mathrm{t}, J=8.4 \mathrm{~Hz}, 2 \mathrm{H}), 4.95-5.02(\mathrm{~m}, 2 \mathrm{H}), 5.05(\mathrm{~d}, J=1.2 \mathrm{~Hz}, 1 \mathrm{H}), 5.22(\mathrm{~s}, 1 \mathrm{H})$, 5.77-5.87 (m, 1H), 6.98-7.02 (m, 2H), 7.33-7.37 (m, 2H); ${ }^{\mathbf{1 3}} \mathbf{C}$ NMR (100 MHz, $\left.\mathrm{CDCl}_{3}\right) \delta 32.5,35.0,112.6,115.0,115.3\left(\mathrm{~d}, J_{C-F}=20.0 \mathrm{~Hz}\right), 127.9\left(\mathrm{~d}, J_{C-F}=10.0 \mathrm{~Hz}\right)$, $137.4\left(\mathrm{~d}, J_{C-F}=10.0 \mathrm{~Hz}\right), 138.1,147.0,161.2,163.6\left(\mathrm{~d}, J_{C-F}=240.0 \mathrm{~Hz}\right) ;{ }^{19} \mathbf{F} \mathbf{~ N M R}$ $\left(376 \mathrm{MHz}, \mathrm{CDCl}_{3}\right) \delta-115.3$. 
1-chloro-4-(hexa-1,5-dien-2-yl)benzene (1i): Known compound. $^{2 b}$ The title<smiles>C=CCCC(=C)c1ccc(Cl)cc1</smiles>
compound was prepared according to the general procedure (Method A) and purified by column chromatography on silica gel and eluted with petroleum ether/ethyl acetate (100/1) to give the colorless oil (1.95 g, $43 \%$ yield). ${ }^{1} \mathbf{H}$ NMR (400 $\left.\mathrm{MHz}, \mathrm{CDCl}_{3}\right) \delta$ 2.16-2.22 (m, 2H), $2.56(\mathrm{t}, J=8.0 \mathrm{~Hz}, 2 \mathrm{H}), 4.95-5.02(\mathrm{~m}, 2 \mathrm{H}), 5.08(\mathrm{~d}, J=1.2 \mathrm{~Hz}, 1 \mathrm{H})$, $5.27(\mathrm{~s}, 1 \mathrm{H}), 5.76-5.87(\mathrm{~m}, 1 \mathrm{H}), 7.27-7.33(\mathrm{~m}, 4 \mathrm{H}) ;{ }^{13} \mathbf{C} \mathbf{N M R}\left(100 \mathrm{MHz}, \mathrm{CDCl}_{3}\right) \delta$ $32.5,34.8,113.1,115.0,127.6,128.5,133.3,138.0,139.8,146.9$.

1-bromo-4-(hexa-1,5-dien-2-yl)benzene (1j): Known compound. $^{2 \mathrm{c}}$ The title<smiles>C=CCCC(=C)c1ccc(Br)cc1</smiles>
compound was prepared according to the general procedure (Method A) and purified by column chromatography on silica gel and eluted with petroleum ether/ethyl acetate (100/1) to give the colorless oil (2.5 g, 58\% yield). ${ }^{1} \mathbf{H} \mathbf{~ N M R}\left(400 \mathrm{MHz}, \mathrm{CDCl}_{3}\right) \delta$ 2.15-2.21 (m, 2H), 2.52-2.56 (m, 2H), 4.94-5.02 (m, 2H), 5.07-5.08 (m, 1H), 5.25-5.26 $(\mathrm{m}, 1 \mathrm{H}), 5.75-5.85(\mathrm{~m}, 1 \mathrm{H}), 7.22-7.26(\mathrm{~m}, 2 \mathrm{H}), 7.41-7.44(\mathrm{~m}, 2 \mathrm{H}) ;{ }^{13} \mathbf{C}$ NMR (100 $\left.\mathrm{MHz}, \mathrm{CDCl}_{3}\right) \delta 32.4,34.7,113.2,115.1,121.4,127.9,131.5,138.0,140.2,146.9$.

4-(hexa-1,5-dien-2-yl)-1,1'-biphenyl (1k): Known compound. ${ }^{2 b}$ The title compound<smiles>C=CCCC(=C)c1ccc(-c2ccccc2)cc1</smiles>
was prepared according to the general procedure (Method A) and purified by column chromatography on silica gel and eluted with petroleum ether/ethyl acetate (100/1) to give the a white solid (2.3 g, 60\% yield). ${ }^{1} \mathbf{H}$ NMR (400 MHz, $\left.\mathrm{CDCl}_{3}\right) \delta 2.23-2.28$ (m, $2 \mathrm{H}), 2.61-2.65(\mathrm{~m}, 2 \mathrm{H}), 4.96-5.05(\mathrm{~m}, 2 \mathrm{H}), 2.61-2.65(\mathrm{~m}, 2 \mathrm{H}), 5.10(\mathrm{~d}, J=1.2 \mathrm{~Hz}, 1 \mathrm{H})$, $5.35(\mathrm{~d}, J=1.2 \mathrm{~Hz}, 1 \mathrm{H}), 5.81-5.91(\mathrm{~m}, 1 \mathrm{H}), 7.32-7.36(\mathrm{~m}, 1 \mathrm{H}), 7.41-7.49(\mathrm{~m}, 4 \mathrm{H})$, 7.55-7.61 (m, 4H); ${ }^{13} \mathrm{C}$ NMR $\left(100 \mathrm{MHz}, \mathrm{CDCl}_{3}\right) \delta 32.6,34.8,112.6,114.9,126.6$, $127.1,127.1,127.4,128.9,138.3,140.2,140.3,140.9,147.5$.

1-(hexa-1,5-dien-2-yl)-4-(trifluoromethyl)benzene (11): Known compound. ${ }^{2 \mathrm{~d}}$ The<smiles>C=CCCC(=C)c1ccc(C(F)(F)F)cc1</smiles>
title compound was prepared according to the general procedure (Method A) and purified by column 
chromatography on silica gel and eluted with petroleum ether/ethyl acetate (50/1) to give the colorless oil (4.0 g, 75\% yield). ${ }^{1} \mathbf{H}$ NMR (400 MHz, $\left.\mathrm{CDCl}_{3}\right) \delta 2.17-2.22$ (m, 2H), $2.60(\mathrm{t}, J=7.6 \mathrm{~Hz}, 2 \mathrm{H}), 4.96-5.02(\mathrm{~m}, 2 \mathrm{H}), 5.16(\mathrm{~d}, J=1.2 \mathrm{~Hz}, 1 \mathrm{H}), 5.33(\mathrm{~s}, 1 \mathrm{H})$ 5.76-5.87 (m, 1H), $7.47(\mathrm{~d}, J=8.0 \mathrm{~Hz}, 2 \mathrm{H}), 7.57(\mathrm{~d}, J=8.2 \mathrm{~Hz}, 2 \mathrm{H}) ;{ }^{13} \mathbf{C} \mathbf{N M R}(100$ $\left.\mathrm{MHz}, \mathrm{CDCl}_{3}\right) \delta 32.4,34.7,114.6,115.2,123.0,125.4\left(\mathrm{q}, J_{C-F}=4.0 \mathrm{~Hz}\right), 126.6,129.5$ $\left(\mathrm{q}, J_{C-F}=32.0 \mathrm{~Hz}\right), 137.8,145.0,147.0 ;{ }^{19} \mathbf{F} \mathbf{~ N M R}\left(376 \mathrm{MHz}, \mathrm{CDCl}_{3}\right) \delta-62.5$.

4-(hexa-1,5-dien-2-yl)benzonitrile (1m): The title compound was prepared according<smiles>C=CCCC(=C)c1ccc(C#N)cc1</smiles>
to the general procedure (Method $\mathbf{A}$ ) and purified by column chromatography on silica gel and eluted with petroleum ether/ethyl acetate (50/1) to give the colorless oil (3.8 g, 97\% yield). ${ }^{1} \mathbf{H}$ NMR (400 MHz, $\left.\mathrm{CDCl}_{3}\right) \delta$ 2.17-2.23 (m, 2H), 2.57-2.61 (m, 2H), 4.96-5.03 (m, 2H), $5.22(\mathrm{~d}, J=1.2 \mathrm{~Hz}, 1 \mathrm{H}), 5.38(\mathrm{~s}, 1 \mathrm{H}), 5.76-5.86(\mathrm{~m}, 1 \mathrm{H}), 7.47-7.50$ (m, 2H), 7.61-7.63 (m, 2H); ${ }^{13} \mathbf{C}$ NMR (100 MHz, $\left.\mathrm{CDCl}_{3}\right) \delta$ 32.3, 34.3, 111.0, 115.3, 115.5, 119.0, 126.9, 132.3, 137.5, 145.9, 146.5. HRMS (ESI) calcd. for $\mathrm{C}_{13} \mathrm{H}_{14} \mathrm{~N}$ [M+H]: 184.1126, found: 184.1122 .

methyl 4-(hexa-1,5-dien-2-yl)benzoate (1n): The title compound was prepared<smiles>C=CCCC(=C)c1ccc(C(=O)OC)cc1</smiles>
according to the general procedure (Method A) and purified by column chromatography on silica gel and eluted with petroleum ether/ethyl acetate (50/1) to give the colorless oil (3.8 g, 84\% yield). ${ }^{1} \mathbf{H}$ NMR $\left(400 \mathrm{MHz}, \mathrm{CDCl}_{3}\right) \delta$ 2.07-2.12 (m, 2H), 2.47-2.51 (m, 2H), $3.80(\mathrm{~s}, 3 \mathrm{H}), 4.85-4.91(\mathrm{~m}, 2 \mathrm{H}), 5.05(\mathrm{~d}, J=1.2 \mathrm{~Hz}, 1 \mathrm{H}), 5.26(\mathrm{~s}, 1 \mathrm{H}), 5.66-5.76$ $(\mathrm{m}, 1 \mathrm{H}), 7.34(\mathrm{~d}, J=8.4 \mathrm{~Hz}, 2 \mathrm{H}), 7.89$ (d, $J=8.4 \mathrm{~Hz}, 2 \mathrm{H}) ;{ }^{13} \mathbf{C}$ NMR $(100 \mathrm{MHz}$, $\left.\mathrm{CDCl}_{3}\right) \delta 32.3,34.5,52.1,114.4,115.0,126.1,129.0,129.7,137.8,145.8,147.1,166.9$. HRMS (ESI) calcd. for $\mathrm{C}_{14} \mathrm{H}_{17} \mathrm{O}_{2}[\mathrm{M}+\mathrm{H}]: 217.1229$, found: 217.1227.

1-(hexa-1,5-dien-2-yl)naphthalene (1p): The title compound was prepared according<smiles>C=CCCC(=C)c1cccc2ccccc12</smiles>
to the general procedure (Method A) and purified by column chromatography on silica gel and eluted with petroleum ether/ethyl acetate (50/1) to give the colorless oil (3.8 g, 84\% 
yield). ${ }^{1} \mathbf{H}$ NMR $\left(400 \mathrm{MHz}, \mathrm{CDCl}_{3}\right) \delta 2.14-2.20(\mathrm{~m}, 2 \mathrm{H}), 2.60(\mathrm{t}, J=8.0 \mathrm{~Hz}, 2 \mathrm{H})$, 4.93-5.01 (m, 2H), 5.09 (s, 1H), 5.40 (t, $J=1.2 \mathrm{~Hz}, 1 \mathrm{H}), 5.76-5.87(\mathrm{~m}, 1 \mathrm{H}), 7.25-7.27$ (m, 1H), 7.38-7.46 (m, 3H), $7.73(\mathrm{~d}, J=8.0 \mathrm{~Hz}, 1 \mathrm{H}), 7.81-7.83(\mathrm{~m}, 3 \mathrm{H}), 8.01-8.04(\mathrm{~m}$, $1 \mathrm{H}) ;{ }^{13} \mathbf{C}$ NMR $\left(100 \mathrm{MHz}, \mathrm{CDCl}_{3}\right) \delta 32.4,38.0,115.0,125.2,125.3,125.8,125.9$, 125.9, 127.3, 128.4, 131.4, 133.8, 138.2, 141.3, 148.3. HRMS (ESI) calcd. for $\mathrm{C}_{16} \mathrm{H}_{17}$ [M+H]: 209.1330, found: 209.1323.

(1S,3s)-1-(hexa-1,5-dien-2-yl)adamantane (1t): The title compound was prepared<smiles>C=CCCC(=C)C12CC3CC(CC(C3)C1)C2</smiles>
according to the general procedure (Method A) and purified by column chromatography on silica gel and eluted with petroleum ether/ethyl acetate $(100 / 1)$ to give the colorless oil $(1.6 \mathrm{~g}, 48 \%$ yield). ${ }^{1} \mathbf{H}$ NMR (400 MHz, $\left.\mathrm{CDCl}_{3}\right) \delta$ 1.64-1.73 (m, 12H), 2.00 (s, 3H), 2.07-2.11 (m, 2H), 2.16-2.22 (m, 2H), $4.71(\mathrm{~d}, J=1.2 \mathrm{~Hz}, 1 \mathrm{H}), 4.81(\mathrm{~s}, 1 \mathrm{H}), 4.92-5.05(\mathrm{~m}, 2 \mathrm{H})$, 5.79-5.89 (m, 1H); ${ }^{13} \mathbf{C}$ NMR $\left(100 \mathrm{MHz}, \mathrm{CDCl}_{3}\right) \delta$ 28.8, 29.6, 33.8, 37.1, 38.0, 41.2, 106.4, 114.4, 139.2, 158.1. HRMS (ESI) calcd. for $\mathrm{C}_{16} \mathrm{H}_{25}[\mathrm{M}+\mathrm{H}]: 217.1956$, found: 217.1960 .

\section{Method B: Synthesis of substrate $10^{1 \mathrm{~b}}$}

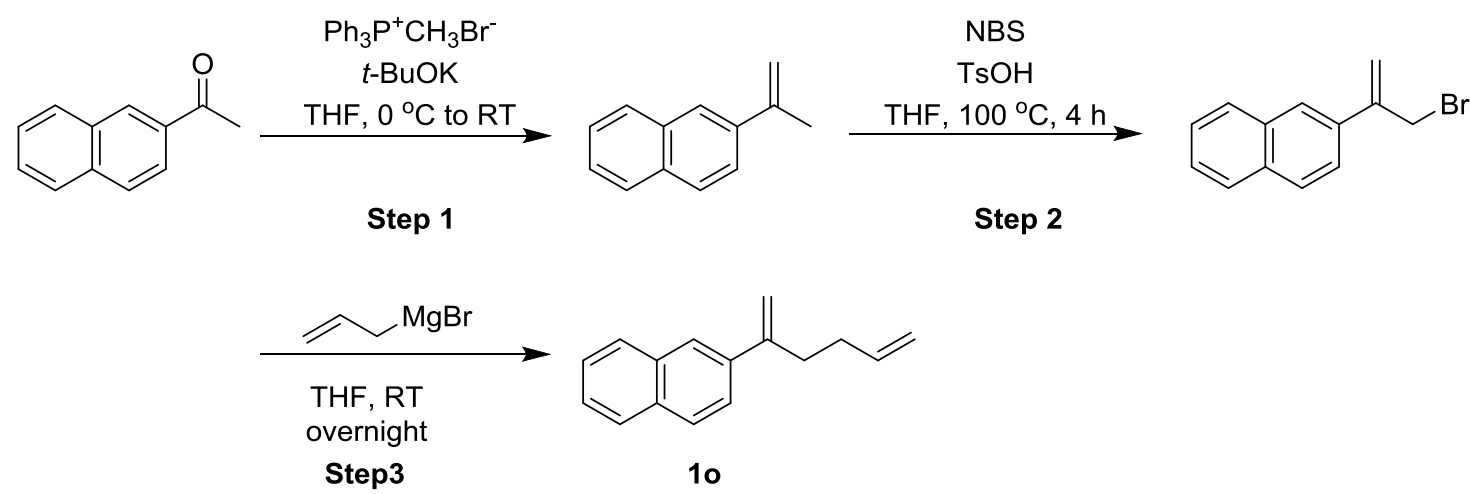

Step 1: In an oven dried flask, methyl triphenylphosphonium bromide (1.2 equiv.) was taken and THF $(1.6 \mathrm{~mL} / \mathrm{mmol})$ was added. The suspension was cooled to $0{ }^{\circ} \mathrm{C}$, $t$-BuOK (1.2 equiv.) was added and the resulting yellow suspension was stirred at $0{ }^{\circ} \mathrm{C}$ for 30 minutes. To this suspension, a solution of ketone (1.0 equiv.) in THF (0.7 $\mathrm{mL} / \mathrm{mmol}$ ) was added dropwise and the resulting mixture was warmed gradually to 
room temperature and stirred overnight. Reaction mixture was concentrated under reduced pressure and filtered over celite. The filtrate was concentrated under reduced pressure to yield yellow oil. The crude product was purified by column chromatography on silica gel and eluted with petroleum ether/ethyl acetate (100/1) to afford 2-(prop-1-en-2-yl)naphthalene (4.6 g, 89\%).

Step 2: In an oven dried flask 2-(prop-1-en-2-yl)naphthalene (1.0 equiv.) was taken and to this dry THF $(3.0 \mathrm{~mL} / \mathrm{mmol})$ was added. To the resulting solution $\mathrm{N}$-Bromosuccinimide (1.05 equiv.) and $\mathrm{TsOH}$ (0.1 equiv.) was added and the solution was refluxed at $100{ }^{\circ} \mathrm{C}$ for 4 hours. Reaction mixture was cooled to room temperature and the reaction mixture was taken in petroleum ether $(15 \mathrm{~mL} / \mathrm{mmol})$, washed with $\mathrm{H}_{2} \mathrm{O}(15 \mathrm{~mL} \times 3)$. Organic phase was dried over $\mathrm{MgSO}_{4}$, concentrated under reduced pressure to obtain a yellow oil. The crude product was purified by column chromatography on silica gel and eluted with petroleum ether/ethyl acetate (100/1) to afford 2-(3-bromoprop-1-en-2-yl)naphthalene (3.2 g, 48\%).

Step 3: To a solution of the 2-(3-bromoprop-1-en-2-yl)naphthalene (5 mmol, 1.0 equiv.) in THF (20 mL) was added $1 \mathrm{M}$ solution of 3-butenylmagnesium bromide in diethyl ether (7.5 mL, $7.5 \mathrm{mmol}, 1.5$ equiv.) at room temperature. The reaction mixture was stirred at room temperature overnight, before it was quenched by adding sat. aq. $\mathrm{NH}_{4} \mathrm{Cl}$ solution. Then the aqueous solution was extracted with diethyl ether three times. The combined organic phases were washed with sat. aq. $\mathrm{NaHCO}_{3}$ solution and brine, dried over $\mathrm{MgSO}_{4}$, filtered and removed in vacuo. The residue was purified by column chromatography on silica gel and eluted with petroleum ether/ethyl acetate (100/1) to afford 2-(hexa-1,5-dien-2-yl)naphthalene 10 as a colorless oil (1.2 g, 44\%).

2-(hexa-1,5-dien-2-yl)naphthalene (10): Known compound. ${ }^{2 b}$ The title compound<smiles>C=CCCC(=C)c1ccc2ccccc2c1</smiles>
was prepared according to the general procedure (Method B) and purified by column chromatography to give the colorless oil (1.2 g, 44\%). ${ }^{1} \mathbf{H}$ NMR (400 MHz, $\left.\mathrm{CDCl}_{3}\right) \delta$ 2.14-2.20 (m, 2H), $2.60(\mathrm{t}, J=8.0 \mathrm{~Hz}, 2 \mathrm{H}), 4.93-5.02(\mathrm{~m}, 2 \mathrm{H}), 5.09(\mathrm{~d}, J=2.0 \mathrm{~Hz}, 1 \mathrm{H}), 5.41(\mathrm{~m}$, $1 \mathrm{H})$, 5.77-5.87 (m, 1H), 7.26-7.28 (m, 2H), 7.39-7.47 (m, 3H), $7.74(\mathrm{~d}, J=8.0 \mathrm{~Hz}, 1 \mathrm{H})$, 
7.81-7.85 (m, 1H), 8.00-8.03 (m, 1H); $\left.{ }^{13} \mathbf{C ~ N M R ~ ( 1 0 0 ~ M H z , ~} \mathrm{CDCl}_{3}\right) \delta 32.4,38.0$, $115.0,115.7,125.2,125.3,125.8,125.9,125.9,127.3,128.4,131.4,133.8,138.3$, $141.3,148.3$.

Procedure C: Synthesis of substrate 1q

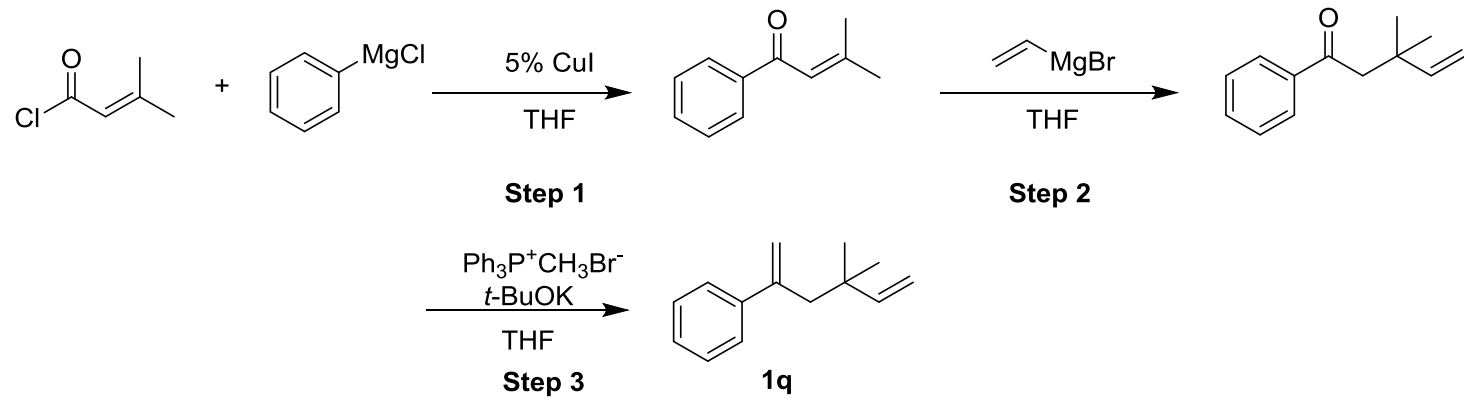

Step 1: To a mixture of the 3-methylbut-2-enoyl chloride (55 mmol, 1.1 equiv.) and $\mathrm{CuI}(465 \mathrm{mg}, 2.5 \mathrm{mmol}, 5 \mathrm{~mol} \%)$ in THF (90 mL) was added $1 \mathrm{M}$ solution of phenylmagnesium chloride in THF $(50 \mathrm{~mL}, 50 \mathrm{mmol}, 1.0$ equiv. $)$ at $-78{ }^{\circ} \mathrm{C}$ dropwise. The reaction mixture was stirred at $-78{ }^{\circ} \mathrm{C}$ for 1 hour and at room temperature overnight, before it was quenched by adding sat. aq. $\mathrm{NH}_{4} \mathrm{Cl}$ solution. Then the aqueous solution was extracted with diethyl ether three times. The combined organic phases were dried over $\mathrm{MgSO}_{4}$, filtered and removed in vacuo. The residue was was purified by column chromatography on silica gel and eluted with petroleum ether/ethyl acetate (50/1) to afford 3-methyl-1-phenylbut-2-en-1-one (5.0 g, 63\% yield).

Step 2: To a solution of the 3-methyl-1-phenylbut-2-en-1-one (30 mmol, 1.0 equiv.) in THF $(60 \mathrm{~mL})$ was added $1 \mathrm{M}$ solution of vinylmagnesium bromide in THF (45 mL, 45 mmol, 1.5 equiv.) at room temperature. The reaction mixture was stirred at room temperature overnight, before it was quenched by adding sat. aq. $\mathrm{NH}_{4} \mathrm{Cl}$ solution. Then the aqueous solution was extracted with diethyl ether three times. The combined organic phases were washed with sat. aq. $\mathrm{NaHCO}_{3}$ solution and brine, dried over $\mathrm{MgSO}_{4}$, filtered and removed in vacuo. The residue was purified by column chromatography on silica gel and eluted with petroleum ether/ethyl acetate (50/1) to afford 3,3-dimethyl-1-phenylpent-4-en-1-one as a colorless oil (4.0 g, 70\%). 
Step 3: To a solution of methyltriphenylphosphonium bromide (25 mmol, 1.25 equiv.) in THF $(80 \mathrm{~mL})$ was added $t$-BuOK $(24 \mathrm{mmol}, 1.2$ equiv. $)$ at $0{ }^{\circ} \mathrm{C}$ and the resulting mixture was stirred for 30 minutes at $0{ }^{\circ} \mathrm{C}$. Then the solution of 3,3-dimethyl-1-phenylpent-4-en-1-one (20 mmol, 1.0 equiv.) in THF (20 mL) was added to the reaction mixture dropwise at $0{ }^{\circ} \mathrm{C}$. After 30 minutes the ice bath was removed and the mixture was stirred overnight at room temperature, before it was quenched by adding sat. aq. $\mathrm{NH}_{4} \mathrm{Cl}$ solution. Then the aqueous solution was extracted with diethyl ether three times. The combined organic phases were dried over $\mathrm{MgSO}_{4}$, filtered and removed in vacuo. The residue was purified by column chromatography on silica gel and eluted with petroleum ether/ethyl acetate (100/1) to afford (4,4-dimethylhexa-1,5-dien-2-yl)benzene 1q (2.6 g, 67\%).

(4,4-dimethylhexa-1,5-dien-2-yl)benzene (1q): The title compound was prepared<smiles>C=CC(C)(C)CC(=C)c1ccccc1</smiles>
according to the general procedure (Method $\mathbf{C}$ ) and purified by column chromatography to give the colorless oil $(2.6 \mathrm{~g}, 67 \%) .{ }^{\mathbf{1}} \mathbf{H}$ NMR $\left(400 \mathrm{MHz}, \mathrm{CDCl}_{3}\right) \delta 0.90(\mathrm{~s}, 6 \mathrm{H}), 2.54$ (s, 2H), 4.75-4.83 $(\mathrm{m}, 2 \mathrm{H}), 5.01(\mathrm{t}, J=0.8 \mathrm{~Hz}, 1 \mathrm{H}), 5.24(\mathrm{~d}, J=2.0 \mathrm{~Hz}, 1 \mathrm{H}), 5.69-5.76(\mathrm{~m}, 1 \mathrm{H})$, 7.20-7.35 (m, 5H); ${ }^{13} \mathbf{C}$ NMR (100 MHz, $\left.\mathrm{CDCl}_{3}\right) \delta$ 27.2, 37.7, 47.9, 109.9, 116.8, 126.8, 127.1, 128.2, 143.6, 146.8, 148.6. HRMS (ESI) calcd. for $\mathrm{C}_{14} \mathrm{H}_{19}[\mathrm{M}+\mathrm{H}]$ : 187.1487, found: 187.1490 .

\section{Procedure D: Synthesis of substrate $1 \mathbf{r}^{1 \mathrm{c}}$}

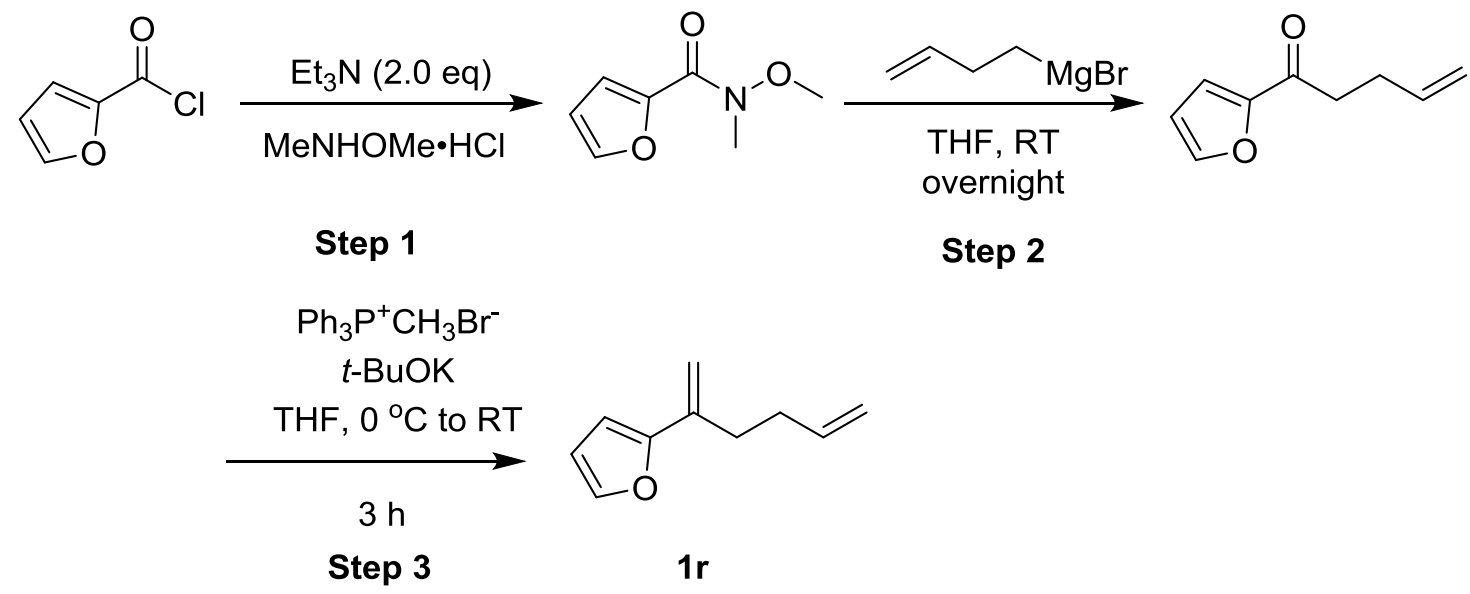


Step 1: Triethylamine (100 mmol, 2.0 equiv.) was slowly added to the mixture of furan-2-carbonyl chloride (50 mmol, 1.0 equiv.) and N,O-dimethylhydroxylamine hydrochloride (52.5 mmol, 1.05 equiv.) in dry $\mathrm{CH}_{2} \mathrm{Cl}_{2}$ at $0{ }^{\circ} \mathrm{C}$. The reaction mixture was allowed to warm up to room temperature and was stirred for 12 hours. After the addition of $\mathrm{HCl}$ (aq., $1.0 \mathrm{~N}, 100 \mathrm{~mL}$ ), the phases were separated and the aqueous layer was extracted with $\mathrm{CH}_{2} \mathrm{Cl}_{2}(40 \times 3 \mathrm{~mL})$. The combined organic phases were dried over $\mathrm{MgSO}_{4}$, filtered and then the solvent was removed in vacuo to afford the Weinreb amide. The crude product was purifiedby column chromatography on silica gel and eluted with petroleum ether/ethyl acetate (2/1) to afford $\mathrm{N}$-methoxy-N-methylfuran-2-carboxamide (6.4 g, 82\%).

Step 2: A solution of 3-butenylmagnesium bromide (0.5 M in THF, $42 \mathrm{mmol}, 1.05$ equiv.) was added over 1 hour to the solution of $\mathrm{N}$-methoxy-N-methylfuran-2-carboxamide (40 mmol, 1.0 equiv.) in dry $\mathrm{THF}$ at $0{ }^{\circ} \mathrm{C}$. The reaction mixture was allowed to warm up to room temperature and was stirred for 3 hours. The $\mathrm{pH}$ of the reaction mixture was adjusted to $\mathrm{pH}=2$ by the addition of $\mathrm{HCl}$ (aq., $1.0 \mathrm{~N}$ ). The phases were separated and the aqueous layer was extracted with diethyl ether $(30 \times 3 \mathrm{~mL})$. The combined organic phases were dried over $\mathrm{MgSO}_{4}$, filtered and the solvent was removed in vacuo. The residue was purified by column chromatography on silica gel and eluted with petroleum ether/ethyl acetate (50/1) to afford 1-(furan-2-yl)pent-4-en-1-one (5 g, 83\%).

Step 3: To a solution of methyltriphenylphosphonium bromide (41 mmol, 1.25 equiv.) in THF was added $t$-BuOK (40 mmol, 1.2 equiv.) at $0{ }^{\circ} \mathrm{C}$ and the resulting mixture was stirred for 30 minutes at $0{ }^{\circ} \mathrm{C}$. Then the solution of 1-(furan-2-yl)pent-4-en-1-one (33 mmol, 1.0 equiv.) in THF was added to the reaction mixture dropwise at $0{ }^{\circ} \mathrm{C}$. After 30 minutes the ice bath was removed and the mixture was stirred overnight at room temperature, before it was quenched by adding sat. aq. $\mathrm{NH}_{4} \mathrm{Cl}$ solution. Then the aqueous solution was extracted with diethyl ether $(30 \times 3 \mathrm{~mL})$. The combined organic phases were dried over $\mathrm{MgSO}_{4}$, filtered and removed in vacuo. The residue was 
purified by column chromatography on silica gel and eluted with petroleum ether/ethyl acetate (100/1) to afford 2-(hexa-1,5-dien-2-yl)furan $1 \mathbf{r}(3.5 \mathrm{~g}, 71 \%)$.

2-(hexa-1,5-dien-2-yl)furan (1r): The title compound was prepared according to the<smiles>C=CCCC(=C)c1ccco1</smiles>
general procedure (Method D) and purified by column chromatography on silica gel and eluted with petroleum ether/ethyl acetate (100/1) to give the colorless oil $(3.5 \mathrm{~g}, 71 \%)$.

${ }^{1}$ H NMR (400 MHz, $\left.\mathrm{CDCl}_{3}\right) \delta$ 2.30-2.35 (m, 2H), 2.43-2.47 (m, 2H), 4.97-5.08 (m, $3 \mathrm{H}), 5.54(\mathrm{~s}, 1 \mathrm{H}), 5.82-5.92(\mathrm{~m}, 1 \mathrm{H}), 6.30(\mathrm{~d}, J=3.2 \mathrm{~Hz}, 1 \mathrm{H}), 6.37-6.38(\mathrm{~m}, 1 \mathrm{H}), 7.35$ $(\mathrm{d}, J=8.0 \mathrm{~Hz}, 1 \mathrm{H}) ;{ }^{13} \mathrm{C}$ NMR $\left(100 \mathrm{MHz}, \mathrm{CDCl}_{3}\right) \delta 32.7,32.9,106.1,109.6,111.2$, 115.0, 137.0, 138.2, 141.9, 154.7. HRMS (ESI) calcd. for $\mathrm{C}_{10} \mathrm{H}_{13} \mathrm{O}[\mathrm{M}+\mathrm{H}]:$ 149.0966, found: 149.0961 .

2-(hexa-1,5-dien-2-yl)thiophene (1s): The title compound was prepared according to<smiles>C=CCCC(=C)c1cccs1</smiles>
the general procedure (Method D) and purified by column chromatography on silica gel and eluted with petroleum ether/ethyl acetate (100/1) to give the colorless oil $(5.8 \mathrm{~g}, 88 \%)$.

${ }^{1} \mathbf{H}$ NMR $\left(400 \mathrm{MHz}, \mathrm{CDCl}_{3}\right) \delta$ 2.30-2.36 (m, 2H), $2.56(\mathrm{t}, J=6.8 \mathrm{~Hz}, 2 \mathrm{H}), 4.97-5.07$ $(\mathrm{m}, 3 \mathrm{H}), 5.41(\mathrm{~s}, 1 \mathrm{H}), 5.82-5.92(\mathrm{~m}, 1 \mathrm{H}), 6.96-6.98(\mathrm{~m}, 1 \mathrm{H}), 7.03-7.04(\mathrm{~m}, 1 \mathrm{H})$, 7.04-7.16 (m, 1H); ${ }^{13} \mathbf{C}$ NMR $\left(100 \mathrm{MHz}, \mathrm{CDCl}_{3}\right) \delta 32.7,35.0,111.2,115.1,123.4$, 124.3, 127.5, 138.1, 141.2, 145.4. HRMS (ESI) calcd. for $\mathrm{C}_{10} \mathrm{H}_{13} \mathrm{~S}[\mathrm{M}+\mathrm{H}]: 165.0738$, found: 165.0732 .

\section{Optimization of the reaction conditions}

In a glove box, a mixture of $\mathrm{Pd}(\mathrm{COD}) \mathrm{Br}_{2}(7.5 \mathrm{mg}, 0.02 \mathrm{mmol}, 5 \mathrm{~mol} \%)$, ligand (0.024 $\mathrm{mmol})$, acid (0.04 mmol, $10 \mathrm{~mol} \%)$, anisole $(1.0 \mathrm{~mL})$ was added into a dry glass vessel. The resulting mixture was stirred for 10 minutes at room temperature and then diene $1 \mathbf{a}(63.2 \mathrm{mg}, 0.4 \mathrm{mmol})$ was added into the reaction mixture. The glass vessel was put into an autoclave and then taken out from glove box. The autoclave was purged and charged with $\mathrm{CO}(20 \mathrm{~atm})$. The reaction mixture was stirred at $80{ }^{\circ} \mathrm{C}$ for 12 hours. After the reaction finished, the autoclave was cooled to room temperature and the 
pressure was carefully released in the fume hood. The combined yield $(\mathbf{2 a}+\mathbf{2 a})$ based on the diene and the ratio $\left(\mathbf{2} \mathbf{a} / \mathbf{2} \mathbf{a}^{\prime}\right)$ of the crude reaction mixture was determined by GC and GC-MS analysis using $n$-dodecane as the internal standard, respectively.

Table S1. Screening of phosphine ligand ${ }^{a}$<smiles>C=CCCC(=C)c1ccccc1</smiles>

\begin{tabular}{|c|c|c|c|c|c|}
\hline Entry & {$[\mathrm{Pd}]$} & Ligand & $\mathrm{CO}(\mathrm{atm})$ & $\frac{\text { Yield }(\%)}{2 \mathbf{a}+\mathbf{2} \mathbf{a}^{\prime}}$ & $2 a^{2} / 2 a^{\prime}$ \\
\hline 1 & $\mathrm{Pd}(\mathrm{COD}) \mathrm{Br}_{2}$ & Xantphos & 20 & 78 & 91:9 \\
\hline 2 & $\mathrm{Pd}(\mathrm{COD}) \mathrm{Br}_{2}$ & DPEphos & 20 & 15 & $88: 12$ \\
\hline 3 & $\mathrm{Pd}(\mathrm{COD}) \mathrm{Br}_{2}$ & DPPF & 20 & 0 & - \\
\hline 4 & $\mathrm{Pd}(\mathrm{COD}) \mathrm{Br}_{2}$ & $\mathrm{DPPH}$ & 20 & 0 & - \\
\hline 5 & $\mathrm{Pd}(\mathrm{COD}) \mathrm{Br}_{2}$ & DPPPen & 20 & 0 & - \\
\hline 6 & $\mathrm{Pd}(\mathrm{COD}) \mathrm{Br}_{2}$ & DPPB & 20 & 0 & - \\
\hline 7 & $\mathrm{Pd}(\mathrm{COD}) \mathrm{Br}_{2}$ & DPPP & 20 & 0 & - \\
\hline 8 & $\mathrm{Pd}(\mathrm{COD}) \mathrm{Br}_{2}$ & DPPE & 20 & 0 & - \\
\hline 9 & $\mathrm{Pd}(\mathrm{COD}) \mathrm{Br}_{2}$ & DPPM & 20 & 0 & - \\
\hline 10 & $\mathrm{Pd}(\mathrm{COD}) \mathrm{Br}_{2}$ & $\mathrm{PPh}_{3}$ & 20 & 21 & $88: 12$ \\
\hline 11 & $\mathrm{Pd}(\mathrm{COD}) \mathrm{Br}_{2}$ & $\mathrm{P}(2-\mathrm{MePh})_{3}$ & 20 & trace & - \\
\hline 12 & $\mathrm{Pd}(\mathrm{COD}) \mathrm{Br}_{2}$ & $\mathrm{P}(4-\mathrm{MePh})_{3}$ & 20 & trace & - \\
\hline 13 & $\mathrm{Pd}(\mathrm{COD}) \mathrm{Br}_{2}$ & $\mathrm{PCy}_{3}$ & 20 & trace & - \\
\hline 14 & $\mathrm{Pd}(\mathrm{COD}) \mathrm{Br}_{2}$ & Ruphos & 20 & trace & - \\
\hline 15 & $\mathrm{Pd}(\mathrm{COD}) \mathrm{Br}_{2}$ & Dave Phos & 20 & trace & - \\
\hline 16 & $\mathrm{Pd}(\mathrm{COD}) \mathrm{Br}_{2}$ & Xphos & 20 & trace & - \\
\hline
\end{tabular}

${ }^{a}$ Reaction conditions: 1a $(0.4 \mathrm{mmol}), \mathrm{Pd}(\mathrm{COD}) \mathrm{Br}_{2}(0.02 \mathrm{mmol}), \mathrm{MsOH}(0.04 \mathrm{mmol}), \mathrm{CO}(20 \mathrm{~atm})$, $80{ }^{\circ} \mathrm{C}$, anisole $(1.0 \mathrm{~mL}), 12 \mathrm{~h}$, bidentate phosphine ligand $(0.024 \mathrm{mmol})$ or monodentate phosphine ligand $(0.04 \mathrm{mmol})$. The combined yield based on the diene and the ratio $(\mathbf{2} \mathbf{a} / \mathbf{2 a})$ of the crude reaction mixture was determined by GC and GC-MS analysis using $n$-dodecane as the internal standard.

Table S2. Screening of palladium ${ }^{a}$<smiles>C=CCCC(=C)c1ccccc1</smiles>

1a
$[\mathrm{Pd}]$

Xantphos $(6 \mathrm{~mol} \%)$

$\mathrm{MsOH}(10 \mathrm{~mol} \%)$

anisole, $80^{\circ} \mathrm{C}, 12 \mathrm{~h}$<smiles>C/C(=C1/CCCC1=O)c1ccccc1</smiles>

2a
$2 \mathbf{a}^{\prime}$

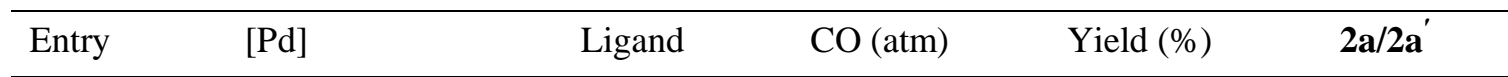




\begin{tabular}{llllcc}
\hline & & \multicolumn{3}{c}{$\mathbf{2 a}+\mathbf{2 a}^{\prime}$} \\
\hline 1 & $\mathrm{Pd}(\mathrm{COD}) \mathrm{Br}_{2}$ & Xantphos & 20 & 78 & $91: 9$ \\
2 & $\mathrm{PdCl}_{2}$ & Xantphos & 20 & 58 & $90: 10$ \\
3 & $\mathrm{Pd}\left(\mathrm{CH}_{3} \mathrm{CN}\right)_{2} \mathrm{Cl}_{2}$ & Xantphos & 20 & 25 & $84: 16$ \\
4 & $\mathrm{Pd}\left(\mathrm{PhCN}_{2} \mathrm{Cl}_{2}\right.$ & Xantphos & 20 & 65 & $88: 12$ \\
5 & $\mathrm{Pd} \mathrm{COD}) \mathrm{Cl}_{2}$ & Xantphos & 20 & 43 & $90: 10$ \\
6 & $\mathrm{PdBr}_{2}$ & Xantphos & 20 & 62 & $89: 11$ \\
$7^{b}$ & $\mathrm{Pd}_{2}(\mathrm{dba})_{3}$ & Xantphos & 20 & trace & - \\
$8^{b}$ & {$[\mathrm{Pd}(\mathrm{allyl}) \mathrm{Cl}]_{2}$} & Xantphos & 20 & trace & - \\
9 & - & Xantphos & 20 & 0 & - \\
\hline
\end{tabular}

${ }^{a}$ Reaction conditions: 1a $(0.4 \mathrm{mmol}),[\mathrm{Pd}](0.02 \mathrm{mmol})$, Xantphos $(0.024 \mathrm{mmol}), \mathrm{MsOH}(0.04$ $\mathrm{mmol}), \mathrm{CO}(20 \mathrm{~atm}), 80^{\circ} \mathrm{C}$, anisole $(1.0 \mathrm{~mL}), 12 \mathrm{~h}$. The combined yield based on the diene and the ratio (2a/2a') of the crude reaction mixture was determined by GC and GC-MS analysis using $n$-dodecane as the internal standard. ${ }^{b}[\mathrm{Pd}](0.01 \mathrm{mmol})$.

Table S3. Screening of acid $^{a}$

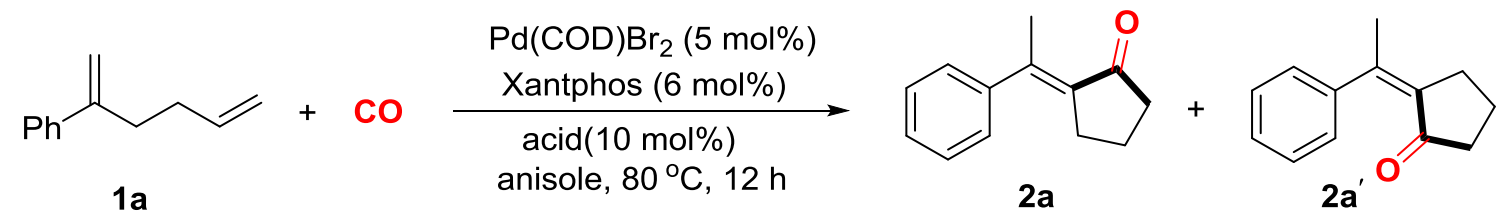

\begin{tabular}{|c|c|c|c|c|c|}
\hline \multirow{2}{*}{ Entry } & \multirow{2}{*}[\mathrm{Pd}]{} & \multirow{2}{*}{ Ligand } & \multirow{2}{*}{ Acid (10 mol\%) } & \multirow{2}{*}{$\begin{array}{c}\text { Yield (\%) } \\
\mathbf{2 a}+\mathbf{2 a}\end{array}$} & \multirow{2}{*}{$2 a / 2 a$} \\
\hline & & & & & \\
\hline 1 & $\mathrm{Pd}(\mathrm{COD}) \mathrm{Br}_{2}$ & Xantphos & $\mathrm{MeSO}_{3} \mathrm{H}$ & 78 & $91: 9$ \\
\hline 2 & $\mathrm{Pd}(\mathrm{COD}) \mathrm{Br}_{2}$ & Xantphos & $\mathrm{CH}_{3} \mathrm{CO}_{2} \mathrm{H}$ & 0 & - \\
\hline 3 & $\mathrm{Pd}(\mathrm{COD}) \mathrm{Br}_{2}$ & Xantphos & $\mathrm{CF}_{3} \mathrm{CO}_{2} \mathrm{H}$ & 22 & $93: 7$ \\
\hline 4 & $\mathrm{Pd}(\mathrm{COD}) \mathrm{Br}_{2}$ & Xantphos & $\mathrm{PivOH}$ & trace & - \\
\hline 5 & $\mathrm{Pd}(\mathrm{COD}) \mathrm{Br}_{2}$ & Xantphos & $\mathrm{PhCO}_{2} \mathrm{H}$ & trace & - \\
\hline 6 & $\mathrm{Pd}(\mathrm{COD}) \mathrm{Br}_{2}$ & Xantphos & $\mathrm{NH}_{2} \mathrm{SO}_{3} \mathrm{H}$ & trace & - \\
\hline 7 & $\mathrm{Pd}(\mathrm{COD}) \mathrm{Br}_{2}$ & Xantphos & $\mathrm{TsOH}$ & 54 & $91: 9$ \\
\hline 8 & $\mathrm{Pd}(\mathrm{COD}) \mathrm{Br}_{2}$ & Xantphos & $\mathrm{PhSO}_{3} \mathrm{H}$ & 73 & $91: 9$ \\
\hline 9 & $\mathrm{Pd}(\mathrm{COD}) \mathrm{Br}_{2}$ & Xantphos & $\mathrm{NMP} \cdot \mathrm{HCl}$ & trace & - \\
\hline 10 & $\mathrm{Pd}(\mathrm{COD}) \mathrm{Br}_{2}$ & Xantphos & $\mathrm{NH}_{2} \mathrm{OCH}_{3} \cdot \mathrm{HCl}$ & 0 & - \\
\hline 11 & $\mathrm{Pd}(\mathrm{COD}) \mathrm{Br}_{2}$ & Xantphos & $\mathrm{NH}_{4} \mathrm{Cl}$ & 0 & - \\
\hline 12 & $\mathrm{Pd}(\mathrm{COD}) \mathrm{Br}_{2}$ & Xantphos & $\mathrm{PhNHNH}_{2} \cdot \mathrm{HCl}$ & 0 & - \\
\hline 13 & $\mathrm{Pd}(\mathrm{COD}) \mathrm{Br}_{2}$ & Xantphos & $\mathrm{Et}_{3} \mathrm{~N} \cdot \mathrm{HCl}$ & 0 & - \\
\hline 14 & $\mathrm{Pd}(\mathrm{COD}) \mathrm{Br}_{2}$ & Xantphos & $\mathrm{EtNH}_{2} \cdot \mathrm{HCl}$ & 0 & - \\
\hline 15 & $\mathrm{Pd}(\mathrm{COD}) \mathrm{Br}_{2}$ & Xantphos & $\mathrm{Et}_{2} \mathrm{NH} \cdot \mathrm{HCl}$ & trace & - \\
\hline 16 & $\mathrm{Pd}(\mathrm{COD}) \mathrm{Br}_{2}$ & Xantphos & $\mathrm{Me}_{2} \mathrm{NH} \cdot \mathrm{HCl}$ & trace & - \\
\hline
\end{tabular}




\begin{tabular}{ccclcc}
\hline 17 & $\mathrm{Pd}(\mathrm{COD}) \mathrm{Br}_{2}$ & Xantphos & $\mathrm{HCl} \cdot \mathrm{NH}_{2} \mathrm{NH}_{2} \cdot \mathrm{H}$ & trace & - \\
18 & $\mathrm{Pd}(\mathrm{COD}) \mathrm{Br}_{2}$ & Xantphos & Glycine & trace & - \\
19 & $\mathrm{Pd}(\mathrm{COD}) \mathrm{Br}_{2}$ & Xantphos & Semicarbazide & trace & - \\
20 & $\mathrm{Pd}(\mathrm{COD}) \mathrm{Br}_{2}$ & Xantphos & $\mathrm{Py} \cdot \mathrm{HCl}$ & trace & - \\
21 & $\mathrm{Pd}(\mathrm{COD}) \mathrm{Br}_{2}$ & Xantphos & $\mathrm{PhNH}_{2} \cdot \mathrm{HCl}$ & 29 & $91: 9$ \\
22 & $\mathrm{Pd}(\mathrm{COD}) \mathrm{Br}_{2}$ & Xantphos & - & 0 & - \\
\hline
\end{tabular}

${ }^{a}$ Reaction conditions: 1a $(0.4 \mathrm{mmol}), \mathrm{Pd}(\mathrm{COD}) \mathrm{Br}_{2}(0.02 \mathrm{mmol})$, Xantphos $(0.024 \mathrm{mmol})$, Acid $(0.04 \mathrm{mmol}, 10 \mathrm{~mol} \%), \mathrm{CO}(20 \mathrm{~atm}), 80{ }^{\circ} \mathrm{C}$, anisole $(1.0 \mathrm{~mL}), 12 \mathrm{~h}$. The combined yield based on the diene and the ratio (2a/2a') of the crude reaction mixture was determined by GC and GC-MS analysis using $n$-dodecane as the internal standard.

\section{Table S4. Screening of solvent ${ }^{a}$}

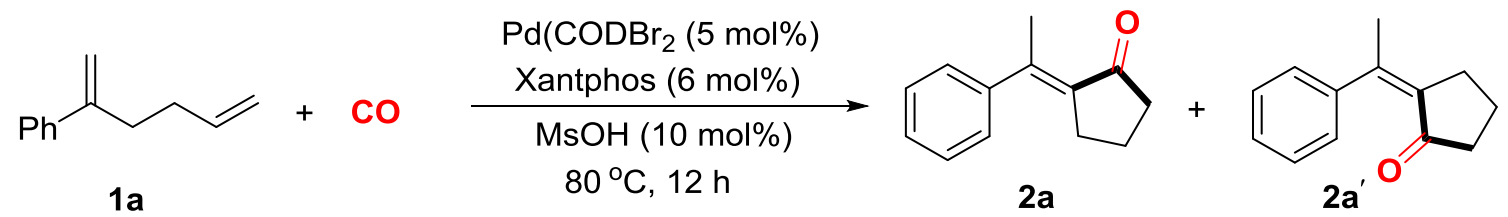

\begin{tabular}{llllcc}
\hline \multirow{2}{*}{ Entry } & \multirow{2}{*}[\mathrm{Pd}]{} & Ligand & Solvent & Yield $(\%)$ & \multirow{2}{*}{$\mathbf{2 a} / \mathbf{2} \mathbf{a}^{\prime}$} \\
\cline { 5 - 5 } & & & & $\mathbf{2 a}+\mathbf{2 a} \mathbf{a}^{\prime}$ & \\
\hline 1 & $\mathrm{Pd}(\mathrm{COD}) \mathrm{Br}_{2}$ & Xantphos & Anisole & 78 & $91: 9$ \\
2 & $\mathrm{Pd}(\mathrm{COD}) \mathrm{Br}_{2}$ & Xantphos & Tol & 76 & $91: 9$ \\
3 & $\mathrm{Pd}(\mathrm{COD}) \mathrm{Br}_{2}$ & Xantphos & Benzene & 70 & $92: 8$ \\
4 & $\mathrm{Pd}(\mathrm{COD}) \mathrm{Br}_{2}$ & Xantphos & $\mathrm{NMP}$ & trace & - \\
5 & $\mathrm{Pd}(\mathrm{COD}) \mathrm{Br}_{2}$ & Xantphos & $\mathrm{DME}$ & trace & - \\
6 & $\mathrm{Pd}(\mathrm{COD}) \mathrm{Br}_{2}$ & Xantphos & $\mathrm{CH}_{3} \mathrm{CN}$ & trace & - \\
7 & $\mathrm{Pd}(\mathrm{COD}) \mathrm{Br}_{2}$ & Xantphos & dioxane & trace & - \\
\hline
\end{tabular}

${ }^{a}$ Reaction conditions: 1a $(0.4 \mathrm{mmol}), \mathrm{Pd}(\mathrm{COD}) \mathrm{Br}_{2}(0.02 \mathrm{mmol})$, Xantphos $(0.024 \mathrm{mmol}), \mathrm{MsOH}$ $(0.04 \mathrm{mmol}), \mathrm{CO}(20 \mathrm{~atm}), 80{ }^{\circ} \mathrm{C}$, Solvent $(1.0 \mathrm{~mL}), 12 \mathrm{~h}$. The combined yield based on the diene and the ratio (2a/2a') of the crude reaction mixture was determined by GC and GC-MS analysis using $n$-dodecane as the internal standard.

Table S5. Screening of temperature ${ }^{a}$

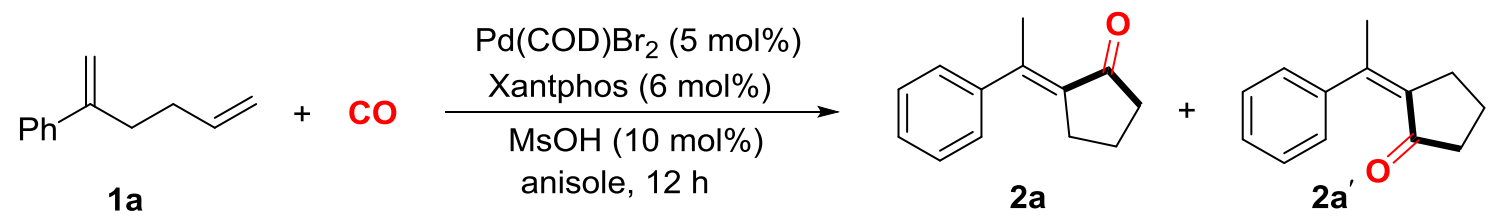

\begin{tabular}{|c|c|c|c|c|c|}
\hline Entry & {$[\mathrm{Pd}]$} & Ligand & $\mathrm{T}\left({ }^{\circ} \mathrm{C}\right)$ & Yield (\%) & $2 \mathrm{a}^{\prime} / 2 \mathrm{a}^{\prime}$ \\
\hline
\end{tabular}




\begin{tabular}{|c|c|c|c|c|c|}
\hline & & & \multicolumn{3}{|c|}{$2 a+2 a^{\prime}$} \\
\hline 1 & $\mathrm{Pd}(\mathrm{COD}) \mathrm{Br}_{2}$ & Xantphos & 100 & 48 & $93: 7$ \\
\hline 2 & $\mathrm{Pd}(\mathrm{COD}) \mathrm{Br}_{2}$ & Xantphos & 80 & 78 & $91: 9$ \\
\hline 3 & $\mathrm{Pd}(\mathrm{COD}) \mathrm{Br}_{2}$ & Xantphos & 60 & 43 & $93: 7$ \\
\hline 4 & $\mathrm{Pd}(\mathrm{COD}) \mathrm{Br}_{2}$ & Xantphos & 40 & 12 & $90: 10$ \\
\hline 5 & $\mathrm{Pd}(\mathrm{COD}) \mathrm{Br}_{2}$ & Xantphos & 25 & 0 & - \\
\hline
\end{tabular}

\section{Table S6. Screening of concentration and catalyst loading ${ }^{a}$}

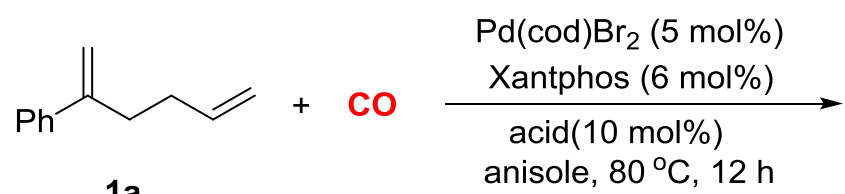

$1 \mathrm{a}$

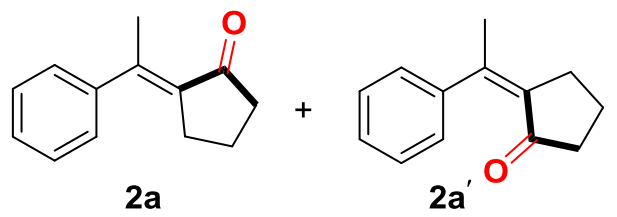

$2 a$
$2 a^{\prime}$

\begin{tabular}{|c|c|c|c|c|c|c|}
\hline \multirow{2}{*}{ Entry } & \multirow{2}{*}[\mathrm{Pd}]{} & \multirow{2}{*}{ Ligand } & \multirow{2}{*}{ anisole $(\mathrm{mL})$} & \multirow{2}{*}{$\mathrm{CO}(\mathrm{atm})$} & Yield(\%) & \multirow{2}{*}{$2 a / 2 a$} \\
\hline & & & & & $2 a+2 a^{\prime}$ & \\
\hline 1 & $\mathrm{Pd}(\mathrm{COD}) \mathrm{Br}_{2}$ & Xantphos & $1 \mathrm{~mL}$ & 20 & 78 & $91: 9$ \\
\hline 2 & $\mathrm{Pd}(\mathrm{COD}) \mathrm{Br}_{2}$ & Xantphos & $2 \mathrm{~mL}$ & 20 & 71 & $92: 8$ \\
\hline 3 & $\mathrm{Pd}(\mathrm{COD}) \mathrm{Br}_{2}$ & Xantphos & $3 \mathrm{~mL}$ & 20 & 69 & $92: 8$ \\
\hline $4^{b}$ & $\mathrm{Pd}(\mathrm{COD}) \mathrm{Br}_{2}$ & Xantphos & $2 \mathrm{~mL}$ & 20 & 74 & 91:9 \\
\hline $5^{b}$ & $\mathrm{Pd}(\mathrm{COD}) \mathrm{Br}_{2}$ & Xantphos & $2 \mathrm{~mL}$ & 30 & 81 & $92: 8$ \\
\hline
\end{tabular}

${ }^{a}$ Reaction conditions: 1a $(0.4 \mathrm{mmol}), \mathrm{Pd}(\mathrm{COD}) \mathrm{Br}_{2}(0.02 \mathrm{mmol})$, Xantphos $(0.024 \mathrm{mmol}), \mathrm{MsOH}$ (0.04 mmol), CO $(20 \mathrm{~atm}), 80{ }^{\circ} \mathrm{C}, 12 \mathrm{~h}$. The combined yield based on the alkene and the ratio (2a/2a') of the crude reaction mixture was determined by GC and GC-MS analysis using $n$-dodecane as the internal standard. ${ }^{b} \mathbf{1 a}(1.0 \mathrm{mmol}), \mathrm{Pd}(\mathrm{COD}) \mathrm{Br}_{2}(0.01 \mathrm{mmol}, 1 \mathrm{~mol} \%)$, Xantphos (0.012 mmol, $1.2 \mathrm{~mol} \%), \mathrm{MsOH}(0.1 \mathrm{mmol}, 10 \mathrm{~mol} \%), \mathrm{CO}(30 \mathrm{~atm}), 90{ }^{\circ} \mathrm{C}$, anisole $(2.0 \mathrm{~mL}), 16$ h. 


\section{General procedure for the catalytic reaction and spectral data of products}

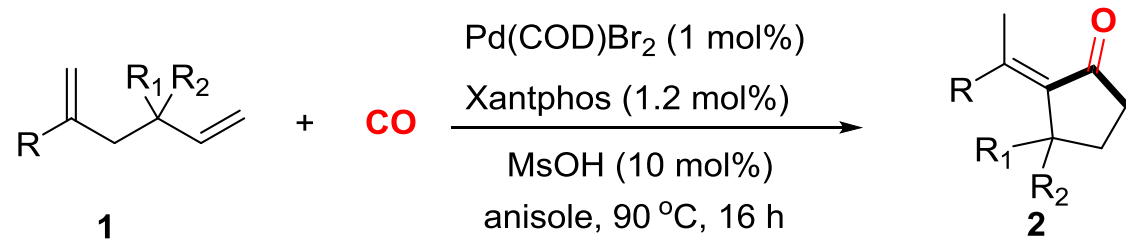

In a glove box, a mixture of $\mathrm{Pd}(\mathrm{COD}) \mathrm{Br}_{2}(3.7 \mathrm{mg}, 0.01 \mathrm{mmol}, 1 \mathrm{~mol} \%)$, Xantphos (6.9 mg, $0.012 \mathrm{mmol}), \mathrm{MsOH}(6.5 \mu \mathrm{L}, 0.1 \mathrm{mmol})$, anisole $(2.0 \mathrm{~mL})$ was added into a dry glass vessel. The resulting mixture was stirred for 10 minutes at room temperature and then diene $1(1.0 \mathrm{mmol})$ was added into the reaction mixture. The glass vessel was put into an autoclave and then taken out from glove box. The autoclave was purged and charged with $\mathrm{CO}(30 \mathrm{~atm})$. The reaction mixture was stirred at $90{ }^{\circ} \mathrm{C}$ for 16 hours. After the reaction finished, the autoclave was cooled to room temperature and the pressure was carefully released in the fume hood. The ratio $(\mathrm{E} / \mathrm{Z})$ of the crude reaction mixture was determined by GC and GC-MS analysis. Then the corresponding reaction mixture was purified by flash column chromatography on silica gel and eluted with petroleum ether/ethyl acetate (100/1-10/1) to give the desired products 2.

(E)-2-(1-phenylethylidene)cyclopentan-1-one (2a): Known compound. ${ }^{3}$ The title compound was prepared according to the general procedure and purified by column

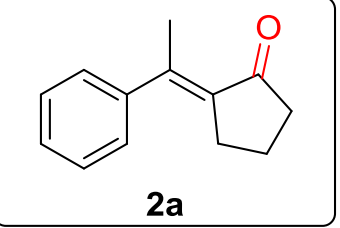
chromatography on silica gel and eluted with petroleum ether/ethyl acetate (100/1-30/1) to give a pale yellow solid, $135.2 \mathrm{mg}, 73 \%$ yield. ${ }^{\mathbf{1}} \mathbf{H}$ NMR (400 MHz, $\left.\mathrm{CDCl}_{3}\right) \delta$ 1.76-1.82 $(\mathrm{m}, 2 \mathrm{H}) 2.38(\mathrm{t}, J=7.6 \mathrm{~Hz}, 2 \mathrm{H}), 2.53(\mathrm{t}, J=2.0 \mathrm{~Hz} 3 \mathrm{H})$, 2.56-2.60 (m, 2H), 7.24-7.25 (m, 2H), 7.26-7.32 (m, 1H), 7.35-7.39 (m, 2H); ${ }^{13} \mathrm{C}$ NMR (100 MHz, $\left.\mathrm{CDCl}_{3}\right) \delta$ 20.1, 20.5, 31.8, 40.7, 127.4, 127.9, 128.3, 132.8, 143.7, 147.7, 209.1. HRMS (ESI) calcd. for $\mathrm{C}_{13} \mathrm{H}_{15} \mathrm{O}[\mathrm{M}+\mathrm{H}]$ : 187.1117, found: 187.1115.

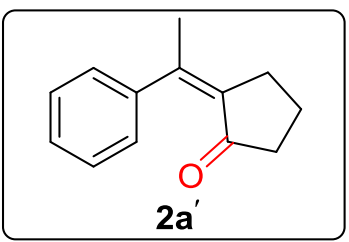

(Z)-2-(1-phenylethylidene)cyclopentan-1-one $\left(2 \mathbf{a}^{\prime}\right)$ : Known compound. ${ }^{5}$ The title compound was prepared according to the 
general procedure and purified by column chromatography on silica gel and eluted with petroleum ether/ethyl acetate (100/1-30/1) to give a pale yellow solid. ${ }^{\mathbf{1}} \mathbf{H}$ NMR $\left(400 \mathrm{MHz}, \mathrm{CDCl}_{3}\right) \delta 1.91-1.99(\mathrm{~m}, 2 \mathrm{H}), 2.11(\mathrm{t}, J=1.6 \mathrm{~Hz}, 3 \mathrm{H}), 2.31(\mathrm{t}, J=8.0 \mathrm{~Hz}$, 2H), 2.74-2.78 (m, 2H), 7.14-7.17 (m, 2H), 7.22-7.36 (m, 3H); ${ }^{13} \mathbf{C}$ NMR (100 MHz, $\left.\mathrm{CDCl}_{3}\right) \delta 19.4,25.0,30.1,40.4,127.4,127.5,127.9,132.2,141.8,146.6,205.1$. HRMS (ESI) calcd. for $\mathrm{C}_{13} \mathrm{H}_{15} \mathrm{O}[\mathrm{M}+\mathrm{H}]:$ 187.1123, found: 187.1122 .

(E)-2-(1-(p-tolyl)ethylidene)cyclopentan-1-one (2b): The title compound was prepared according to the general procedure and purified by column chromatography

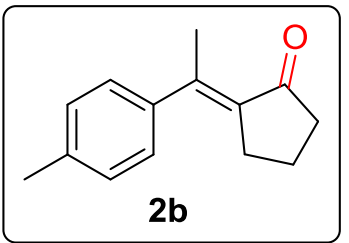
on silica gel and eluted with petroleum ether/ethyl acetate (100/1-30/1) to give the pale yellow oil, $146.1 \mathrm{mg}, 73 \%$ yield. ${ }^{1} \mathbf{H}$ NMR $\left(400 \mathrm{MHz}, \mathrm{CDCl}_{3}\right) \delta$ 1.74-1.82 (m, 2H), 2.35-2.39 (m, $5 \mathrm{H}), 2.52(\mathrm{t}, J=2.0 \mathrm{~Hz}, 3 \mathrm{H}), 2.57-2.61(\mathrm{~m}, 2 \mathrm{H}), 7.15-7.19(\mathrm{~m}$, $4 \mathrm{H}) ;{ }^{13} \mathrm{C}$ NMR $\left(100 \mathrm{MHz}, \mathrm{CDCl}_{3}\right) \delta$ 19.9, 20.4, 21.3, 31.9, 40.6, 127.3, 128.9, 132.4, 137.8, 140.6, 147.6, 208.9. HRMS (ESI) calcd. for $\mathrm{C}_{14} \mathrm{H}_{17} \mathrm{O}[\mathrm{M}+\mathrm{H}]$ : 201.1274, found: 201.1276.

(E)-2-(1-(m-tolyl)ethylidene)cyclopentan-1-one (2c): The title compound was

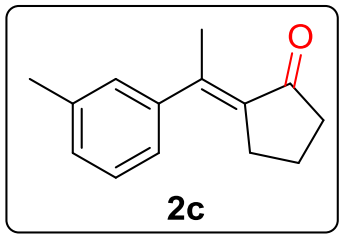
prepared according to the general procedure and purified by column chromatography on silica gel and eluted with petroleum ether/ethyl acetate (100/1-30/1) to give the pale yellow oil, $141.7 \mathrm{mg}, 71 \%$ yield. ${ }^{1} \mathbf{H}$ NMR $\left(400 \mathrm{MHz}, \mathrm{CDCl}_{3}\right) \delta 1.75-1.82$ $(\mathrm{m}, 2 \mathrm{H}), 2.35-2.39(\mathrm{~m}, 5 \mathrm{H}), 2.52(\mathrm{t}, J=2.0 \mathrm{~Hz}, 3 \mathrm{H}), 2.55-2.60(\mathrm{~m}, 2 \mathrm{H}), 7.03-7.06(\mathrm{~m}$, 2H), 7.10-7.13 (m, 1H), 7.24-7.27 (m, 1H); ${ }^{13}$ C NMR (100 MHz, $\left.\mathrm{CDCl}_{3}\right) \delta 20.1,20.4$, $21.5,31.8,40.6,124.4,127.9,128.2,128.6,132.5,137.9,143.6,147.8,209.0$. HRMS (ESI) calcd. for $\mathrm{C}_{14} \mathrm{H}_{17} \mathrm{O}$ [M+H]: 201.1274, found: 201.1275.

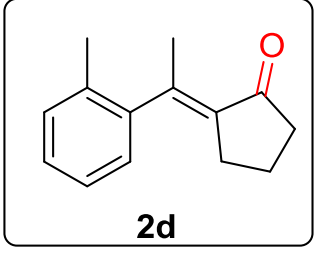

(E)-2-(1-(o-tolyl)ethylidene)cyclopentan-1-one (2d): The title compound was prepared according to the general procedure and 
purified by column chromatography on silica gel and eluted with petroleum ether/ethyl acetate (100/1-30/1) to give the pale yellow oil, $101.0 \mathrm{mg}, 50 \%$ yield. ${ }^{1} \mathbf{H}$ NMR (400 $\left.\mathrm{MHz}, \mathrm{CDCl}_{3}\right) \delta 1.76-1.84(\mathrm{~m}, 2 \mathrm{H}), 2.19-2.23(\mathrm{~m}, 4 \mathrm{H}), 2.26-2.33(\mathrm{~m}, 1 \mathrm{H}), 2.37-2.42(\mathrm{~m}$, 5H), 6.94-6.98 (m, 1H), 7.17-7.22 (m, 3H); $\left.{ }^{13} \mathbf{C ~ N M R ~ ( 1 0 0 ~ M H z , ~} \mathrm{CDCl}_{3}\right) \delta 19.1,20.0$, 20.3, 30.6, 40.8, 126.1, 126.1, 127.3, 130.4, 132.9, 133.2, 143.6, 148.7, 208.8. HRMS (ESI) calcd. for $\mathrm{C}_{14} \mathrm{H}_{17} \mathrm{O}$ [M+H]: 201.1274, found: 201.1275.

(E)-2-(1-(4-ethylphenyl)ethylidene)cyclopentan-1-one (2e): The title compound was

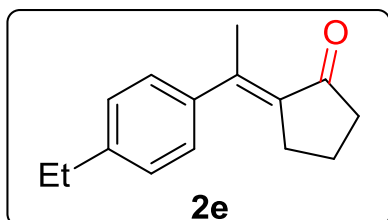

$2 \mathrm{e}$ prepared according to the general procedure and purified by column chromatography on silica gel and eluted with petroleum ether/ethyl acetate (100/1-30/1) to give the pale yellow oil, $162.4 \mathrm{mg}, 76 \%$ yield. ${ }^{1} \mathbf{H}$ NMR (400 MHz, $\left.\mathrm{CDCl}_{3}\right) \delta 1.25(\mathrm{t}, J=7.6 \mathrm{~Hz}, 3 \mathrm{H}), 1.75-1.82(\mathrm{~m}, 2 \mathrm{H}), 2.37(\mathrm{t}, J=7.6 \mathrm{~Hz}, 2 \mathrm{H}), 2.52(\mathrm{t}, J$ $=2.0 \mathrm{~Hz}, 3 \mathrm{H}), 2.58-2.69(\mathrm{~m}, 4 \mathrm{H}), 7.20-7.26(\mathrm{~m}, 4 \mathrm{H}) ;{ }^{13} \mathbf{C} \mathbf{~ N M R}\left(100 \mathrm{MHz}, \mathrm{CDCl}_{3}\right) \delta$ $15.5,20.0,20.6,28.7,32.1,40.7,127.5,127.7,132.5,140.8,144.2,209.2$. HRMS (ESI) calcd. for $\mathrm{C}_{15} \mathrm{H}_{19} \mathrm{O}[\mathrm{M}+\mathrm{H}]: 215.1430$, found: 215.1430 .

(E)-2-(1-(4-(tert-butyl)phenyl)ethylidene)cyclopentan-1-one (2f): The title

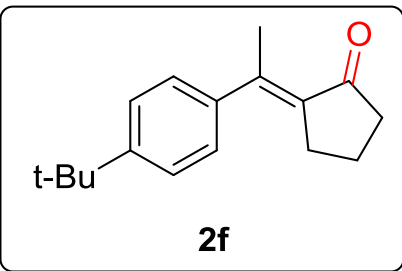

compound was prepared according to the general procedure and purified by column chromatography on silica gel and eluted with petroleum ether/ethyl acetate (100/1-30/1) to give a pale yellow solid, $178.2 \mathrm{mg}, 74 \%$ yield. ${ }^{1} \mathbf{H}$ NMR $\left(400 \mathrm{MHz}, \mathrm{CDCl}_{3}\right) \delta 1.34(\mathrm{~s}, 9 \mathrm{H}), 1.75-1.83(\mathrm{~m}, 2 \mathrm{H}), 2.38$ $(\mathrm{t}, J=7.6 \mathrm{~Hz}, 2 \mathrm{H}), 2.53(\mathrm{t}, J=2.0 \mathrm{~Hz}, 3 \mathrm{H}), 2.61-2.65(\mathrm{~m}, 2 \mathrm{H}), 7.21-7.23(\mathrm{~m}, 2 \mathrm{H})$, 7.37-7.41 (m, 2H); ${ }^{13} \mathbf{C}$ NMR $\left(100 \mathrm{MHz}, \mathrm{CDCl}_{3}\right) \delta$ 19.9, 20.6, 31.4, 32.2, 34.8, 40.7, 125.1, 127.3, 132.5, 140.5, 147.6, 151.1, 209.2. HRMS (ESI) calcd. for $\mathrm{C}_{17} \mathrm{H}_{23} \mathrm{O}$ [M+H]: 243.1743, found: 243.1746. 
(E)-2-(1-(4-methoxyphenyl)ethylidene)cyclopentan-1-one (2g): The title compound 2g

was prepared according to the general procedure and purified by column chromatography on silica gel and eluted with petroleum ether/ethyl acetate (100/1-10/1) to give a pale yellow solid, $116.5 \mathrm{mg}$, 54\% yield. ${ }^{1} \mathbf{H}$ NMR (400 MHz, $\left.\mathrm{CDCl}_{3}\right) \delta 1.75-1.82(\mathrm{~m}, 2 \mathrm{H}), 2.37(\mathrm{t}, J=7.6 \mathrm{~Hz}, 2 \mathrm{H}), 2.52(\mathrm{t}, J=2.0 \mathrm{~Hz}, 3 \mathrm{H})$, 2.60-2.65 (m, 2H), $3.83(\mathrm{~s}, 3 \mathrm{H}), 6.88-6.92(\mathrm{~m}, 2 \mathrm{H}), 7.22-7.27(\mathrm{~m}, 2 \mathrm{H}) ;{ }^{13}$ C NMR $(100$ $\left.\mathrm{MHz}, \mathrm{CDCl}_{3}\right) \delta 19.9,20.6,32.2,40.6,55.4,113.6,129.1,132.2,135.8,147.2,159.4$, 208.9. HRMS (ESI) calcd. for $\mathrm{C}_{14} \mathrm{H}_{17} \mathrm{O}_{2}[\mathrm{M}+\mathrm{H}]: 217.1228$, found: 217.1226.

The compound was also confirmed by single-crystal X-ray analysis

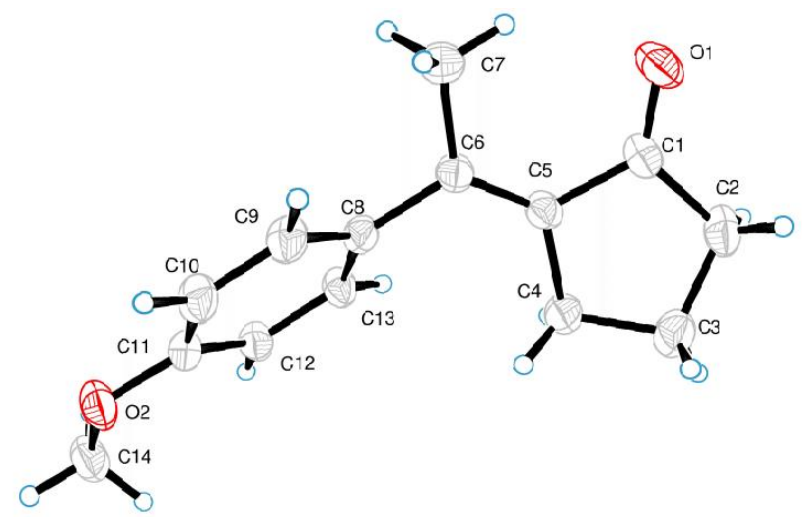

Figure S1. ORTEP drawing of complex $\mathbf{2 g}$

(E)-2-(1-(4-fluorophenyl)ethylidene)cyclopentan-1-one (2h): The title compound

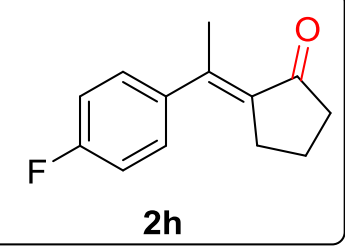

was prepared according to the general procedure and purified by column chromatography on silica gel and eluted with petroleum ether/ethyl acetate (100/1-20/1) to give the pale yellow oil, $110.2 \mathrm{mg}, 54 \%$ yield. ${ }^{1} \mathbf{H}$ NMR $\left(400 \mathrm{MHz}, \mathrm{CDCl}_{3}\right)$ $\delta 1.77-1.84(\mathrm{~m}, 2 \mathrm{H}), 2.39(\mathrm{t}, J=7.2 \mathrm{~Hz}, 2 \mathrm{H}), 2.51(\mathrm{t}, J=2.0 \mathrm{~Hz}, 3 \mathrm{H}), 2.54-2.59(\mathrm{~m}$, 2H), 7.03-7.09 (m, 2H), 7.22-7.26 (m, 2H); ${ }^{13} \mathbf{C}$ NMR (100 MHz, $\left.\mathrm{CDCl}_{3}\right) \delta 20.1,20.5$, 31.9, 40.6, $115.4\left(\mathrm{~d}, J_{C-F}=20.0 \mathrm{~Hz}\right), 129.3\left(\mathrm{~d}, J_{C-F}=10.0 \mathrm{~Hz}\right), 133.0,139.6\left(\mathrm{~d}, J_{C-F}=\right.$ $10.0 \mathrm{~Hz}), 146.5,163.5\left(\mathrm{~d}, J_{C-F}=240.0 \mathrm{~Hz}\right), 208.9 ;{ }^{19} \mathbf{F} \mathbf{N M R}\left(376 \mathrm{MHz}, \mathrm{CDCl}_{3}\right) \delta$ -113.62. HRMS (ESI) calcd. for $\mathrm{C}_{13} \mathrm{H}_{14} \mathrm{FO}[\mathrm{M}+\mathrm{H}]: 205.1029$, found: 205.1024 . 
(E)-2-(1-(4-chlorophenyl)ethylidene)cyclopentan-1-one (2i): The title compound

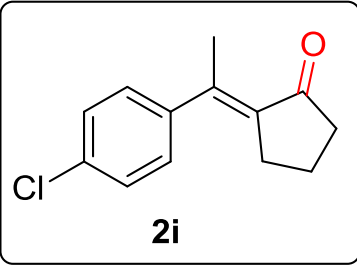

was prepared according to the general procedure and purified by column chromatography on silica gel and eluted with petroleum ether/ethyl acetate (100/1-20/1) to give the pale yellow oil, $136.5 \mathrm{mg}, 62 \%$ yield. ${ }^{1} \mathbf{H}$ NMR $\left(400 \mathrm{MHz}, \mathrm{CDCl}_{3}\right)$ $\delta 1.77-1.84(\mathrm{~m}, 2 \mathrm{H}), 2.38(\mathrm{t}, J=8.0 \mathrm{~Hz}, 2 \mathrm{H}), 2 . .50(\mathrm{t}, J=2.0 \mathrm{~Hz}, 3 \mathrm{H}), 2.53-2.58(\mathrm{~m}$, 2H), 7.18-7.21 (m, 2H), 7.33-7.36 (m, 2H); $\left.{ }^{13} \mathbf{C ~ N M R ~ ( 1 0 0 ~ M H z , ~} \mathrm{CDCl}_{3}\right) \delta 19.8,20.4$, 31.8, 40.5, 128.5, 128.8, 133.1, 133.7, 141.9, 146.1, 208.7. HRMS (ESI) calcd. for $\mathrm{C}_{13} \mathrm{H}_{14} \mathrm{ClO}[\mathrm{M}+\mathrm{H}]: 221.0728$, found: 221.0728 .

(E)-2-(1-(4-bromophenyl)ethylidene)cyclopentan-1-one (2j): The title compound

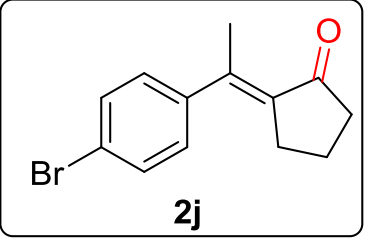

was prepared according to the general procedure and purified by column chromatography on silica gel and eluted with petroleum ether/ethyl acetate (100/1-20/1) to give a pale yellow solid, $158.8 \mathrm{mg}, 60 \%$ yield. ${ }^{1} \mathbf{H}$ NMR (400 MHz, $\left.\mathrm{CDCl}_{3}\right) \delta 1.77-1.84(\mathrm{~m}, 2 \mathrm{H}), 2.38(\mathrm{t}, J=7.6 \mathrm{~Hz}, 2 \mathrm{H}), 2.49(\mathrm{t}, J=2.0 \mathrm{~Hz}, 3 \mathrm{H})$, 2.53-2.58 (m, 2H), 7.12-7.15 (m, 2H), 7.48-7.52 (m, 2H); ${ }^{13} \mathbf{C ~ N M R ~ ( 1 0 0 ~ M H z , ~} \mathrm{CDCl}_{3}$ ) $\delta 19.7,20.3,31.7,40.5,121.9,129.1,131.5,133.1,142.4,146.0,208.7$. HRMS (ESI) calcd. for $\mathrm{C}_{13} \mathrm{H}_{13} \mathrm{BrNaO}$ [M+Na]: 287.0042, found: 287.0037 .

(E)-2-(1-([1,1'-biphenyl]-4-yl)ethylidene)cyclopentan-1-one $\quad(2 \mathrm{k}): \quad$ The title

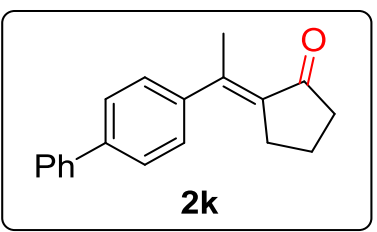

compound was prepared according to the general procedure and purified by column chromatography on silica gel and eluted with petroleum ether/ethyl acetate (100/1-30/1) to give a white solid, $163.1 \mathrm{mg}, 62 \%$ yield. ${ }^{1} \mathbf{H}$ NMR (400 MHz, $\left.\mathrm{CDCl}_{3}\right) \delta 1.77-1.84(\mathrm{~m}, 2 \mathrm{H}), 2.39(\mathrm{t}, J=7.6 \mathrm{~Hz}, 2 \mathrm{H}), 2.56(\mathrm{t}, J=2.0 \mathrm{~Hz}, 3 \mathrm{H})$, 2.62-2.66 (m, 2H), 7.32-7.37 (m, 3H), 7.42-7.46 (m, 2H), 7.58-7.62 (m, 4H); ${ }^{13} \mathrm{C}$ NMR $\left(100 \mathrm{MHz}, \mathrm{CDCl}_{3}\right) \delta 19.9,20.6,32.1,40.7,127.0,127.2,127.7,128.0,129.0$, 
132.9, 140.6, 140.8, 142.4, 147.1, 209.1. HRMS (ESI) calcd. for $\mathrm{C}_{19} \mathrm{H}_{18} \mathrm{NaO}[\mathrm{M}+\mathrm{Na}]$ : 285.1250, found: 285.1248 .

(E)-2-(1-(4-(trifluoromethyl)phenyl)ethylidene)cyclopentan-1-one (2I): The title

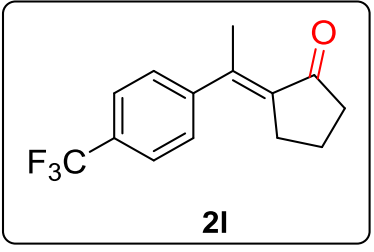

compound was prepared according to the general procedure and purified by column chromatography on silica gel and eluted with petroleum ether/ethyl acetate (100/1-10/1) to give a pale yellow solid, $117.6 \mathrm{mg}, 46 \%$ yield. ${ }^{\mathbf{1}} \mathbf{H}$ NMR (400 MHz, $\left.\mathrm{CDCl}_{3}\right) \delta 1.79-1.86(\mathrm{~m}, 2 \mathrm{H}), 2.41(\mathrm{t}, J=8.0 \mathrm{~Hz}, 2 \mathrm{H}), 2.52-2.56(\mathrm{~m}, 5 \mathrm{H}), 7.36(\mathrm{q}, J=$ $8.0 \mathrm{~Hz}, 2 \mathrm{H}), 7.64(\mathrm{q}, J=8.0 \mathrm{~Hz}, 2 \mathrm{H}) ;{ }^{13} \mathbf{C} \mathbf{N M R}\left(100 \mathrm{MHz}, \mathrm{CDCl}_{3}\right) \delta 19.8,20.3,31.6$, 40.6, 122.8, $125.4\left(\mathrm{~d}, J_{C-F}=4.0 \mathrm{~Hz}\right), 129.9\left(\mathrm{~d}, J_{C-F}=32.0 \mathrm{~Hz}\right), 130.1,130.4,133.7$, 145.8, 147.2, 208.8. HRMS (ESI) calcd. for $\mathrm{C}_{14} \mathrm{H}_{13} \mathrm{~F}_{3} \mathrm{NaO}$ [M+Na]: 277.0811, found: 277.0808 .

(E)-4-(1-(2-oxocyclopentylidene)ethyl)benzonitrile (2m): The title compound was

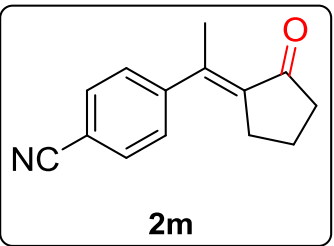
prepared according to the general procedure and purified by column chromatography on silica gel and eluted with petroleum ether/ethyl acetate (100/1-10/1) to give a pale yellow solid, 69.8 mg, $33 \%$ yield. ${ }^{1} \mathbf{H}$ NMR (400 $\left.\mathrm{MHz}, \mathrm{CDCl}_{3}\right) \delta$ 1.80-1.87 (m, 2H), $2.41(\mathrm{t}, J=7.6 \mathrm{~Hz}, 2 \mathrm{H}), 2.51-2.55(\mathrm{~m}, 5 \mathrm{H}), 7.35-7.37(\mathrm{~d}, J=8.0 \mathrm{~Hz}, 2 \mathrm{H})$, 7.68-7.70 (m, 2H); ${ }^{13} \mathrm{C}$ NMR $\left(100 \mathrm{MHz}, \mathrm{CDCl}_{3}\right) \delta$ 19.6, 20.3, 31.6, 40.5, 111.6, 118.7, 128.2, 132.3, 134.0, 145.0, 148.2, 208.6. HRMS (ESI) calcd. for $\mathrm{C}_{14} \mathrm{H}_{13} \mathrm{NNaO}$ [M+Na]: 234.0889, found: 234.0888 .

Methyl (E)-4-(1-(2-oxocyclopentylidene)ethyl)benzoate (2n): The title compound

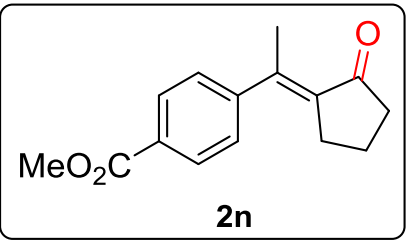
was prepared according to the general procedure and purified by column chromatography on silica gel and eluted with petroleum ether/ethyl acetate (100/1-10/1) to give a pale yellow solid, $138.3 \mathrm{mg}, 57 \%$ yield. ${ }^{\mathbf{1}} \mathbf{H}$ NMR (400 $\left.\mathrm{MHz}, \mathrm{CDCl}_{3}\right) \delta 1.78-1.85(\mathrm{~m}, 2 \mathrm{H}), 2.39(\mathrm{t}, J=8.0 \mathrm{~Hz}, 2 \mathrm{H}), 2.53-2.67(\mathrm{~m}, 5 \mathrm{H}), 3.93(\mathrm{~d}$, 
$J=0.8 \mathrm{~Hz}, 3 \mathrm{H}), 7.31-7.33(\mathrm{~m}, 2 \mathrm{H}), 8.04-8.06(\mathrm{~m}, 2 \mathrm{H}) ;{ }^{13} \mathbf{C ~ N M R}\left(100 \mathrm{MHz}, \mathrm{CDCl}_{3}\right) \delta$ $19.8,20.4,31.6,40.6,52.3,127.4,129.5,129.7,133.4,146.3,148.2,166.8,208.9$. HRMS (ESI) calcd. for $\mathrm{C}_{15} \mathrm{H}_{16} \mathrm{NaO}_{3}$ [M+Na]: 267.0992, found: 267.0989.

(E)-2-(1-(naphthalen-2-yl)ethylidene)cyclopentan-1-one (2o): The title compound

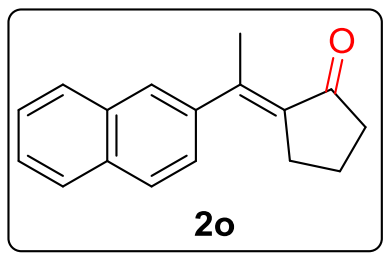

was prepared according to the general procedure and purified by column chromatography on silica gel and eluted with petroleum ether/ethyl acetate (100/1-30/1) to give a pale yellow solid, $156.0 \mathrm{mg}, 66 \%$ yield. ${ }^{1} \mathbf{H}$ NMR $(400 \mathrm{MHz}$, $\left.\mathrm{CDCl}_{3}\right) \delta 1.76-1.83(\mathrm{~m}, 2 \mathrm{H}), 2.40(\mathrm{t}, J=7.6 \mathrm{~Hz}, 2 \mathrm{H}), 2.58-2.64(\mathrm{~m}, 5 \mathrm{H}), 7.36-7.38(\mathrm{~m}$, 1H), 7.47-7.51 (m, 2H), 7.69-7.70 (m, 1H), 7.81-7.84 (m, 3H); ${ }^{13} \mathbf{C}$ NMR (100 MHz, $\left.\mathrm{CDCl}_{3}\right) \delta 20.1,20.5,31.9,40.6,125.4,126.5,127.8,128.0,128.2,132.8,133.1,133.1$, 141.0, 147.5, 208.9. HRMS (ESI) calcd. for $\mathrm{C}_{17} \mathrm{H}_{16} \mathrm{NaO}$ [M+Na]: 259.1093, found: 259.1086.

(E)-2-(1-(naphthalen-1-yl)ethylidene)cyclopentan-1-one (2p): The title compound

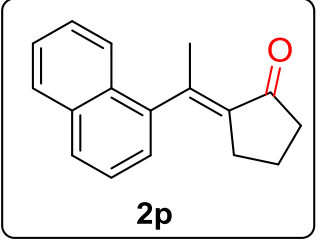
was prepared according to the general procedure and purified by column chromatography on silica gel and eluted with petroleum ether/ethyl acetate (100/1-30/1) to give a pale yellow solid, 117.9 $\mathrm{mg}, 50 \%$ yield. ${ }^{1} \mathbf{H}$ NMR $\left(400 \mathrm{MHz}, \mathrm{CDCl}_{3}\right) \delta 1.71-1.79(\mathrm{~m}, 2 \mathrm{H})$, 2.12-2.29(m, 2H), $2.43(\mathrm{t}, J=7.6 \mathrm{~Hz}, 2 \mathrm{H}), 2.60(\mathrm{t}, J=2.0 \mathrm{~Hz}, 3 \mathrm{H}), 7.19-7.21(\mathrm{~m}$, 1H), .7.45-7.51 (m, 3H), 7.70-7.72 (m, 1H), 7.79 (d, $J=8.4 \mathrm{~Hz}, 1 \mathrm{H}), 7.86-7.89(\mathrm{~m}, 1 \mathrm{H})$;

${ }^{13}$ C NMR $\left(100 \mathrm{MHz}, \mathrm{CDCl}_{3}\right) \delta 19.9,21.1,30.8,40.8,123.6,125.0,125.7,126.1$, 126.5, 127.7, 128.7, 129.2, 133.8, 134.3, 141.8, 147.3, 208.9. HRMS (ESI) calcd. for $\mathrm{C}_{17} \mathrm{H}_{16} \mathrm{NaO}[\mathrm{M}+\mathrm{Na}]: 259.1093$, found: 259.1092 .

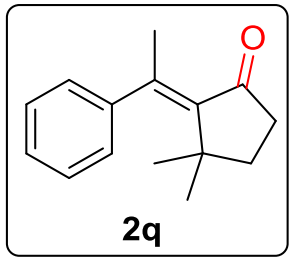

(E)-3,3-dimethyl-2-(1-phenylethylidene)cyclopentan-1-one (2q): The title compound was prepared according to the general procedure and purified by column chromatography on silica gel 
and eluted with petroleum ether/ethyl acetate (100/1-30/1) to give a pale yellow solid, $120.1 \mathrm{mg}, 56 \%$ yield. ${ }^{1} \mathbf{H}$ NMR $\left(400 \mathrm{MHz}, \mathrm{CDCl}_{3}\right) \delta 0.87(\mathrm{~s}, 6 \mathrm{H}), 1.65(\mathrm{t}, J=7.6 \mathrm{~Hz}$, 2H), $2.38(\mathrm{t}, J=8.0 \mathrm{~Hz}, 2 \mathrm{H}), 2.44(\mathrm{~s}, 3 \mathrm{H}), 7.08-7.11(\mathrm{~m}, 2 \mathrm{H}), 7.27-7.36(\mathrm{~m}, 3 \mathrm{H}) ;{ }^{13} \mathrm{C}$ NMR $\left(100 \mathrm{MHz}, \mathrm{CDCl}_{3}\right) \delta 24.1,29.2,37.1,37.4,42.0,127.0,127.2,128.0,140.8$, 143.8, 149.9, 210.2. HRMS (ESI) calcd. for $\mathrm{C}_{15} \mathrm{H}_{19} \mathrm{O}[\mathrm{M}+\mathrm{H}]$ : 215.1430, found: 215.1434 .

(E)-2-(1-(furan-2-yl)ethylidene)cyclopentan-1-one (2r): The title compound was

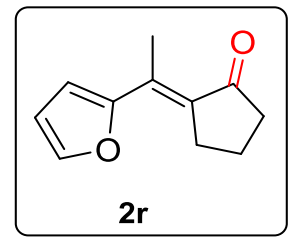
prepared according to the general procedure and purified by column chromatography on silica gel and eluted with petroleum ether/ethyl acetate (100/1-20/1) to give the pale yellow oil, $75.4 \mathrm{mg}, 43 \%$ yield.

${ }^{1} \mathbf{H}$ NMR $\left(400 \mathrm{MHz}, \mathrm{CDCl}_{3}\right) \delta 1.89-1.97(\mathrm{~m}, 2 \mathrm{H}), 2.38(\mathrm{t}, J=8.0 \mathrm{~Hz}$, $2 \mathrm{H}), 2.54(\mathrm{t}, J=2.0 \mathrm{~Hz}, 3 \mathrm{H}), 2.95-2.99(\mathrm{~m}, 2 \mathrm{H}), 6.51\left(\mathrm{dd}, J_{1}=3.6 \mathrm{~Hz}, J_{2}=1.6 \mathrm{~Hz}, 1 \mathrm{H}\right)$, $6.69(\mathrm{~d}, J=3.6 \mathrm{~Hz}, 1 \mathrm{H}) 7.55(\mathrm{~d}, J=1.6 \mathrm{~Hz}, 1 \mathrm{H}) ;{ }^{13} \mathbf{C ~ N M R}\left(100 \mathrm{MHz}, \mathrm{CDCl}_{3}\right) \delta 14.9$, 20.0, 32.4, 40.5, 112.1, 114.2, 129.7, 134.0, 144.0, 155.1, 209.5. HRMS (ESI) calcd. for $\mathrm{C}_{11} \mathrm{H}_{13} \mathrm{O}_{2}[\mathrm{M}+\mathrm{H}]:$ 177.0916, found: 177.0899.

(E)-2-(1-(thiophen-2-yl)ethylidene)cyclopentan-1-one (2s): The title compound was

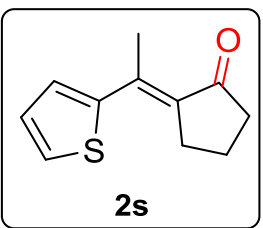

prepared according to the general procedure and purified by column chromatography on silica gel and eluted with petroleum ether/ethyl acetate (100/1-20/1) to give a pale yellow solid, $108.0 \mathrm{mg}, 56 \%$ yield. ${ }^{1} \mathbf{H}$ NMR $\left(400 \mathrm{MHz}, \mathrm{CDCl}_{3}\right) \delta$ 1.90-1.97 (m, 2H), $2.41(\mathrm{t}, J=8.0 \mathrm{~Hz}$, 2H), $2.68(\mathrm{t}, J=2.0 \mathrm{~Hz}, 3 \mathrm{H}), 2.92-2.96(\mathrm{~m}, 2 \mathrm{H}), 7.11-7.14(\mathrm{~m}, 1 \mathrm{H}), 7.34-7.35(\mathrm{~m}, 1 \mathrm{H})$, 7.46-7.47 (m, 1H); ${ }^{13} \mathrm{C}$ NMR $\left(100 \mathrm{MHz}, \mathrm{CDCl}_{3}\right) \delta 19.0,20.2,33.2,40.7,127.5,128.1$, 129.2, 131.0, 139.2, 145.7, 208.9. HRMS (ESI) calcd. for $\mathrm{C}_{11} \mathrm{H}_{12} \mathrm{ONaS}[\mathrm{M}+\mathrm{H}]$ : 215.0509, found: 215.0494 .

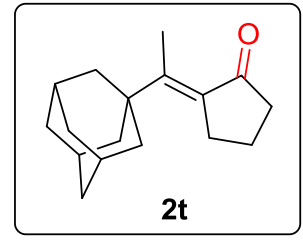

(E)-2-(1-((1S,3s)-adamantan-1-yl)ethylidene)cyclopentan-1-one (2t): The title compound was prepared according to the general 
procedure and purified by column chromatography on silica gel and eluted with petroleum ether/ethyl acetate (100/1-30/1) to give the pale yellow oil, $67.1 \mathrm{mg}, 28 \%$ yield. ${ }^{1} \mathbf{H}$ NMR (400 MHz, $\left.\mathrm{CDCl}_{3}\right) \delta 1.71$ (br, 6H), 1.78-1.84 (m, 2H), 1.89-1.99 (br, $6 \mathrm{H}), 2.0(\mathrm{~s}, 3 \mathrm{H}), 2.20-2.25(\mathrm{~m}, 5 \mathrm{H}), 2.86-2.90(\mathrm{~m}, 2 \mathrm{H}) ;{ }^{13} \mathbf{C ~ N M R}\left(100 \mathrm{MHz}, \mathrm{CDCl}_{3}\right) \delta$ 15.7, 20.5, 28.9, 32.2, 36.9 39.4, 39.7, 40.7, 131.4, 159.3, 210.5. HRMS (ESI) calcd. for $\mathrm{C}_{17} \mathrm{H}_{25} \mathrm{O}[\mathrm{M}+\mathrm{H}]: 245.1900$, found: 245.1892 .

\section{Gram-scale synthesis of $2 a$}

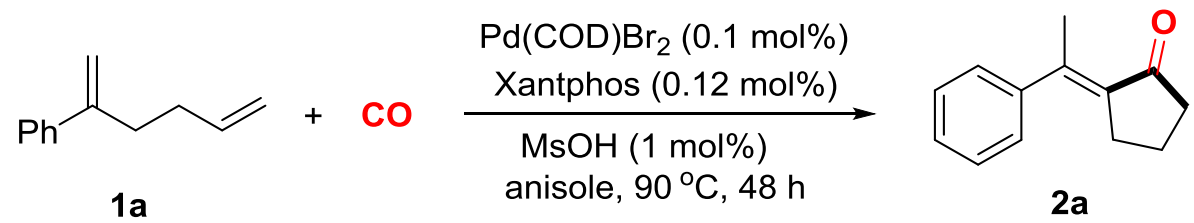

In a glove box, a mixture of $\operatorname{Pd}(\mathrm{COD}) \mathrm{Br}_{2}(3.7 \mathrm{mg}, 0.01 \mathrm{mmol}, 0.1 \mathrm{~mol} \%)$, Xantphos (6.9 mg, $0.012 \mathrm{mmol}, 0.12 \mathrm{~mol} \%), \mathrm{MsOH}(6.5 \mu \mathrm{L}, 0.1 \mathrm{mmol}, 1 \mathrm{~mol} \%)$, anisole $(10.0 \mathrm{~mL})$ was added into a $50 \mathrm{~mL}$ round-bottomed flask. The resulting mixture was stirred for 10 minutes at room temperature and then 1,5-diene $1 \mathbf{a}(10 \mathrm{mmol})$ was added into the reaction mixture. The round-bottomed flask was put into an autoclave and then taken out from glove box. The autoclave was purged and charged with $\mathrm{CO}$ (30 atm). The reaction mixture was stirred at $90{ }^{\circ} \mathrm{C}$ for 48 hours. After the reaction finished, the autoclave was cooled to room temperature and the pressure was carefully released in the fume hood, solvent was removed under reduced pressure. The ratio $\left(\mathbf{2 a} / \mathbf{2} \mathbf{a}^{\prime}\right)$ of the crude reaction mixture was determined by GC and GC-MS. Then the corresponding reaction mixture was purified by flash column chromatography on silica gel and eluted with petroleum ether/ethyl acetate (100/1-30/1) to give the desired products $\mathbf{2 a}\left(910.1 \mathrm{mg}, 49 \%\right.$ yield), $\mathbf{2} \mathbf{a} / \mathbf{2} \mathbf{a}^{\prime}=91: 9$.

\section{Mechanistic studies}

\subsection{Reaction of 1a under $\mathrm{MsOH}$}

$\mathrm{MsOH}(6.5 \mu \mathrm{L}, 0.1 \mathrm{mmol}), 1,5$-diene $1 \mathrm{a}(1.0 \mathrm{mmol})$, anisole $(2.0 \mathrm{~mL})$ were added to a $25 \mathrm{~mL}$ flame-dried Young-type tube under nitrogen atmosphere. The reaction 
mixture was stirred for 16 hour at $90{ }^{\circ} \mathrm{C}$. After the reaction finished, the solvent was removed under reduced pressure. The combined yield $\left(\mathbf{A}+\mathbf{A}^{\prime}\right)$ based on the diene and the ratio $\left(\mathbf{A} / \mathbf{A}^{\prime}\right)$ of the crude reaction mixture was determined by GC and GC-MS analysis using $n$-dodecane as the internal standard. Then the corresponding reaction mixture was purified by flash column chromatography on silica gel and eluted with petroleum to give the desired products $\mathbf{A}+\mathbf{A}^{\prime}$.

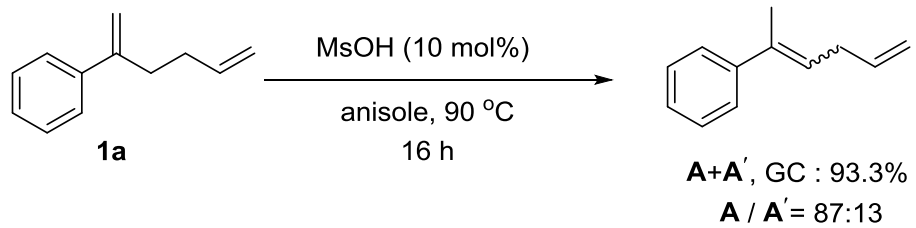

Hexa-2,5-dien-2-ylbenzene $\left(\mathbf{A}+\mathbf{A}^{\prime}\right)$ : A colorless oil. $(\boldsymbol{E})$-hexa-2,5-dien-2-ylbenzene

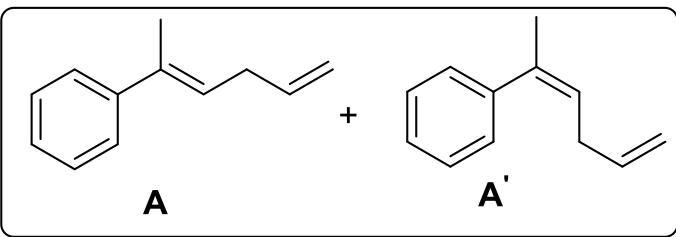

(A): Known compound. ${ }^{4}{ }^{\mathbf{1}} \mathbf{H}$ NMR (400 $\left.\mathrm{MHz}, \mathrm{CDCl}_{3}\right) \quad \delta: \quad 2.03-2.04(\mathrm{~m}, \quad 3 \mathrm{H})$, 2.94-2.97 (m, 2H), 5.00-5.12 (m, 2H), 5.77-5.93 (m, 2H), 7.20-7.40 (m, 5H);

(Z)-hexa-2,5-dien-2-ylbenzene (A'): Known compound. ${ }^{4}{ }^{1} \mathbf{H}$ NMR (400 MHz, $\left.\mathrm{CDCl}_{3}\right) \delta:$ 2.04-2.05 (m, 3H), 2.70-2.74 (m, 2H), 4.95-5.05 (m, 2H), 5.47-5.51 (m, $1 \mathrm{H})$, 5.76-5.86 (m, 1H), 7.17-7.34 (m, 5H).

\subsection{Cyclocarbonylation of $1,4-d i e n e ~\left(A+A^{\prime}\right)$}

In a glove box, a mixture of $\mathrm{Pd}(\mathrm{COD}) \mathrm{Br}_{2}(3.7 \mathrm{mg}, 0.01 \mathrm{mmol}, 1 \mathrm{~mol} \%)$, Xantphos (6.9 mg, $0.012 \mathrm{mmol}), \mathrm{MsOH}(6.5 \mu \mathrm{L}, 0.1 \mathrm{mmol})$, anisole $(2 \mathrm{~mL})$ was added into a dry glass vessel. The resulting mixture was stirred for 10 minutes at room temperature and then 1,4-diene $\left(\mathbf{A}: \mathbf{A}^{\prime}=\mathbf{8 1 : 1 9}\right)(1.0 \mathrm{mmol})$ was added into the reaction mixture. The glass vessel was put into an autoclave and then taken out from glove box. The autoclave was purged and charged with $\mathrm{CO}(30 \mathrm{~atm})$. The reaction mixture was stirred at $90{ }^{\circ} \mathrm{C}$ for 16 hours. After the reaction finished, the autoclave was cooled to room temperature and the pressure was carefully released in the fume hood. The ratio $\left(\mathbf{2} \mathbf{a} / \mathbf{2} \mathbf{a}^{\prime}\right)$ 
of the crude reaction mixture was determined by GC and GC-MS. Then the corresponding reaction mixture was purified by flash column chromatography on silica gel and eluted with petroleum ether/ethyl acetate (100/1-30/1) to give the desired products $\mathbf{2 a}$ (111.4 $\mathrm{mg}, 60 \%$ yield), $\mathbf{2 a} / \mathbf{2} \mathbf{a}^{\prime}=91: 9$.

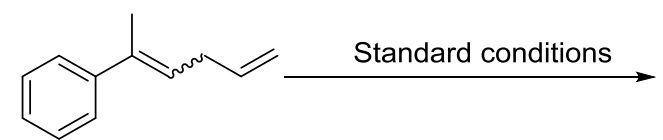

$\mathbf{A}: \mathbf{A}^{\prime}=81: 19$

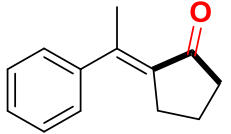

$2 a, 60 \%$

\subsection{Reaction profile}

In a glove box, a mixture of $\mathrm{Pd}(\mathrm{COD}) \mathrm{Br}_{2}(11.1 \mathrm{mg}, 0.03 \mathrm{mmol}, 1 \mathrm{~mol} \%)$, Xantphos (20.7 mg, $0.036 \mathrm{mmol}), \mathrm{MsOH}(19.2 \mu \mathrm{L}, 0.1 \mathrm{mmol})$, anisole $(15 \mathrm{~mL})$ was added into a PTFE vessel. The resulting mixture was stirred for 10 minutes at room temperature and then diene 1a $(3.0 \mathrm{mmol})$ was added into the reaction mixture. The PTFE vessel was put into an autoclave and then taken out from glove box. The autoclave was purged and charged with $\mathrm{CO}(30 \mathrm{~atm})$. The reaction mixture was stirred at $90{ }^{\circ} \mathrm{C}$. Yields were determined by GC and GC-MS analysis using $n$-dodecane as the internal standard.

Table S7 Reaction profile of Pd-catalyzed hydrocarbonylative cyclization of $\mathbf{1 a}$.

\begin{tabular}{|c|c|c|c|c|}
\hline$t(\min )$ & $\mathbf{1 a}(\%)$ & $\mathbf{A}(\%)$ & $\mathbf{2 a}(\%)$ & $\mathbf{A}^{\prime}(\%)$ \\
\hline 0 & 100 & 0 & 0 & 0 \\
\hline 20 & 94.5 & 5.5 & 0 & 0 \\
\hline 60 & 74.5 & 19.4 & 2.3 & 1.0 \\
\hline 100 & 58.7 & 31.5 & 2.7 & 1.1 \\
\hline 140 & 47.5 & 40.0 & 2.7 & 2.0 \\
\hline 200 & 26.0 & 55.7 & 6.3 & 2.3 \\
\hline 260 & 17.6 & 59.3 & 9.3 & 2.6 \\
\hline 320 & 13.7 & 59.3 & 11.9 & 2.6 \\
\hline 380 & 10.5 & 55.5 & 17.5 & 2.8 \\
\hline 440 & 8.1 & 52.9 & 21.2 & 2.7 \\
\hline 500 & 6.6 & 46.2 & 28.0 & 2.6 \\
\hline 560 & 5.6 & 40.8 & 33.2 & 2.5 \\
\hline 620 & 4.9 & 38.2 & 36.7 & 2.3 \\
\hline 680 & 4.2 & 32.8 & 42.2 & 2.2 \\
\hline 740 & 3.6 & 29.5 & 45.2 & 2.0 \\
\hline 800 & 3.2 & 25.1 & 49.5 & 1.7 \\
\hline 860 & 2.5 & 20.5 & 54.3 & 1.7 \\
\hline 920 & 2.4 & 19.4 & 55.5 & 1.7 \\
\hline 980 & 2.3 & 17.9 & 57.0 & 1.6 \\
\hline & & & & \\
\hline
\end{tabular}




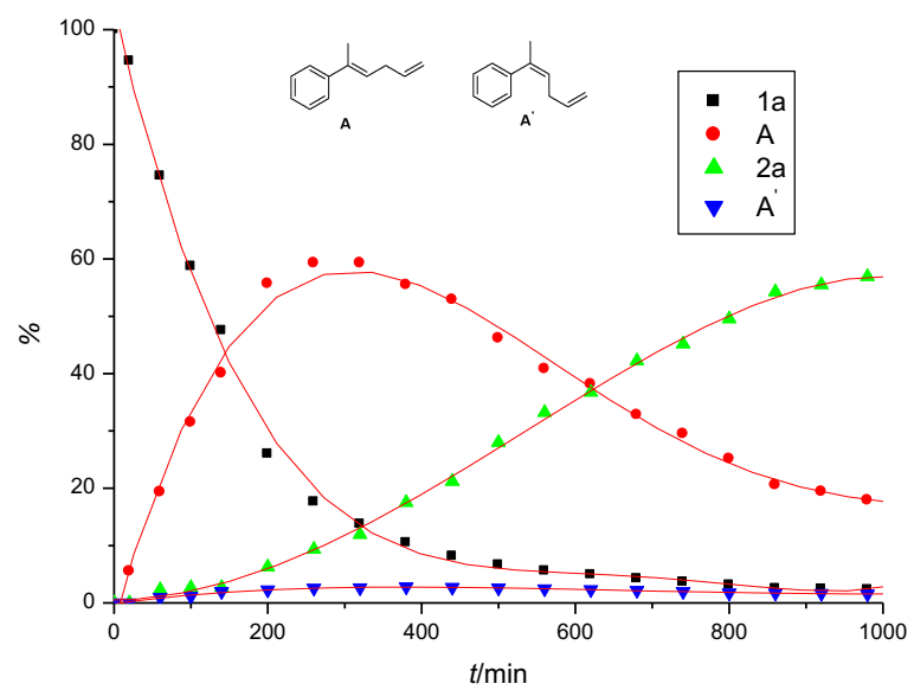

Figure S2 Reaction profile of Pd-catalyzed hydrocarbonylative cyclization of $\mathbf{1 a}$.

\section{References}

(1) (a) Imai, M.; Tanaka, M.; Tanaka, K.; Yamamoto, Y.; Imai-Ogata, N.; Shimowatari, M.; Nagumo, S.; Kawahara, No.; Suemune, H. Double-Chelation-Assisted Rh-Catalyzed Intermolecular Hydroacylation between Salicylaldehydes and 1,4-Pentaor 1,5-Hexadienes. J. Org. Chem. 2004, 69, 1144. (b) Jiang, B.; Meng, F.; Liang, Q.; Xu, Y.; Loh, T-P. Palladium-Catalyzed Direct Intramolecular C-N Bond Formation: Access to Multisubstituted Dihydropyrroles. Org. Lett. 2017, 19, 914. (c) Uehara, K. B.; Wagner, C.; Vogler, T.; Luftmann, H.; Studer, A. Poly(vinyl ketone)s by Controlled Boron Group Transfer Polymerization (BGTP). Angew. Chem. Int. Ed. 2010, 49, 3073.

(2) (a) Ikeda, H.; Minegishi, T.; Miyashi, T.; Lakkaraju, P. S.; Sauers, R. R.; Roth, H.

D. Incorporation of 2-Arylhexa-1,5-diene into Pentasil Zeolite: A Distorted 1-Arylcyclohexane-1,4-diyl Radical Cation at Room Temperature. J. Phys. Chem. B, 2005, 109, 2504. (b) Xu, S.; Wang, C.; Komiyama, M.; Tomonari, Y.; Negishi, E-i. 
Asymmetric Synthesis of Chiral Cyclopentanes Bearing an All - Carbon Quaternary Stereocenter by Zirconium - Catalyzed Double Carboalumination. Angew. Chem. Int. Ed. 2017, 56, 11502. (c) Li, W.; Yuan, W.; Shi, M.; Hernandez, E.; Li, G. Rhodium(I)-Catalyzed Intramolecular Ene Reaction of Vinylidenecyclopropanes and Alkenes for the Formation of Bicyclo[5.1.0]octylenes. Org. Lett. 2010, 12, 64. (d) Overman, L. E.; Renaldo, A. E. Catalyzed Sigmatropic Rearrangements. 10. Mechanism of the Palladium Dichloride Catalyzed Cope Rearrangement of Acyclic Dienes. A Substituent Effect Study. J. Am. Chem. Soc. 1990, 112, 3945.

(3) Larock, R. C.; Reddy, C. K. Synthesis of 2-Alkylidenecyclopentanones via Palladium-Catalyzed Carbopalladation/Ring Expansion of 1-(1-Alkynyl)cyclobutanols. J. Org. Chem. 2002, 67, 2027.

(4) Matsubara, R.; Jamison, T. F. Nickel-Catalyzed Allylic Substitution of Simple Alkenes. J. Am. Chem. Soc. 2010, 132, 6880.

(5) Thottumkara, A. P.; Kurokawa, T.; Du Bois, J. Carbocyclization of Unsaturated Thioesters under Palladium Catalysis. Chem. Sci. 2013, 4, 2686.

\section{Copies for ${ }^{1} \mathrm{H}$ NMR, ${ }^{19} \mathrm{~F}$ NMR and ${ }^{13} \mathrm{C}$ NMR of materials and products}


ZSC-X180810-3-CDC13-HNMR-1a
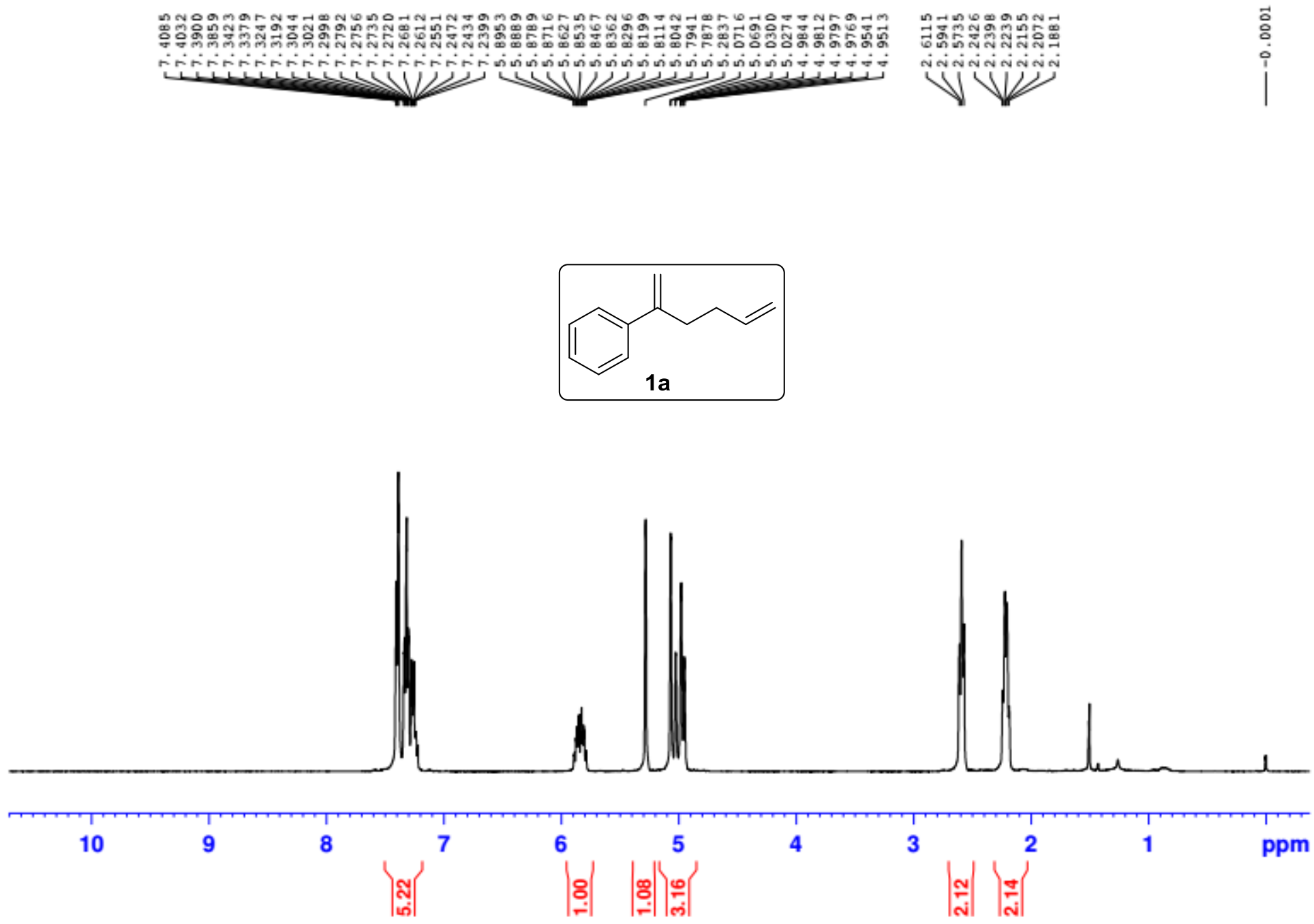
ZSC-X180810-3-CDC13-HNMR-1a
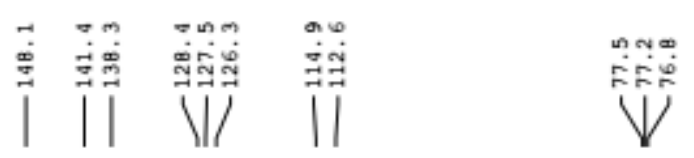

\$ंग

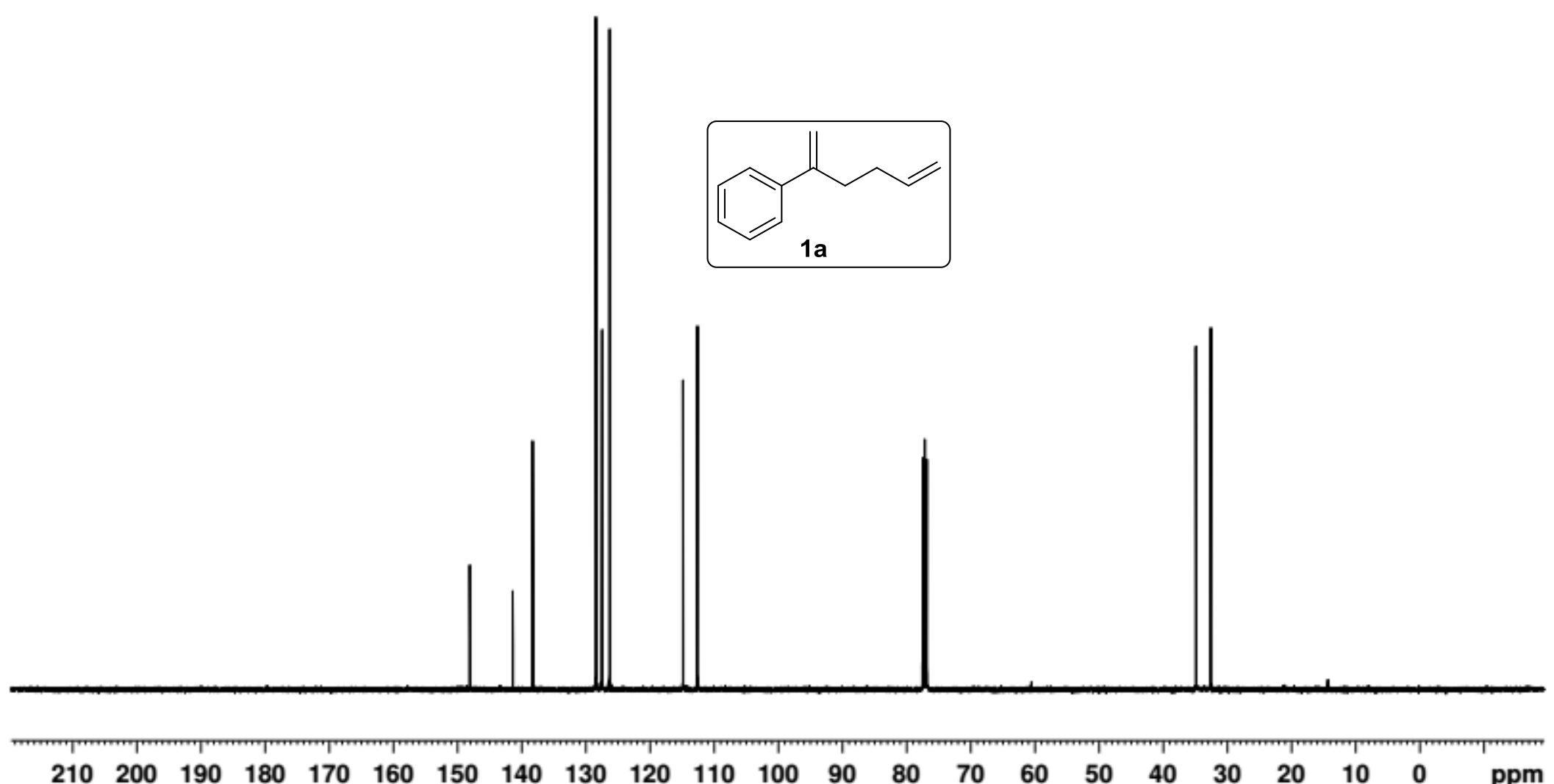



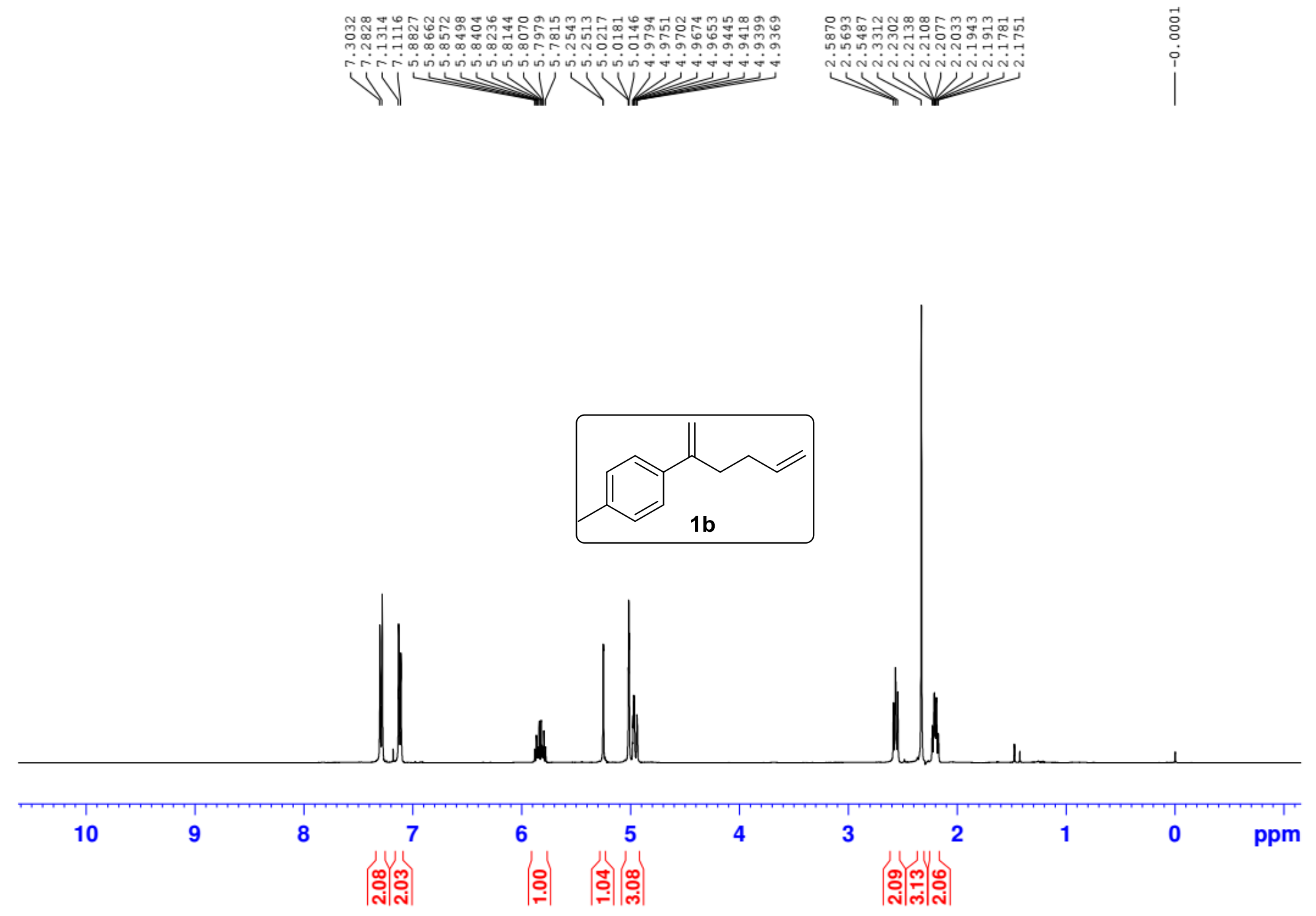
HY-X180122-1-CDC13-CNMR

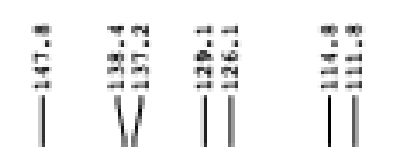

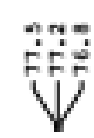

|⿱一𫝀山亍

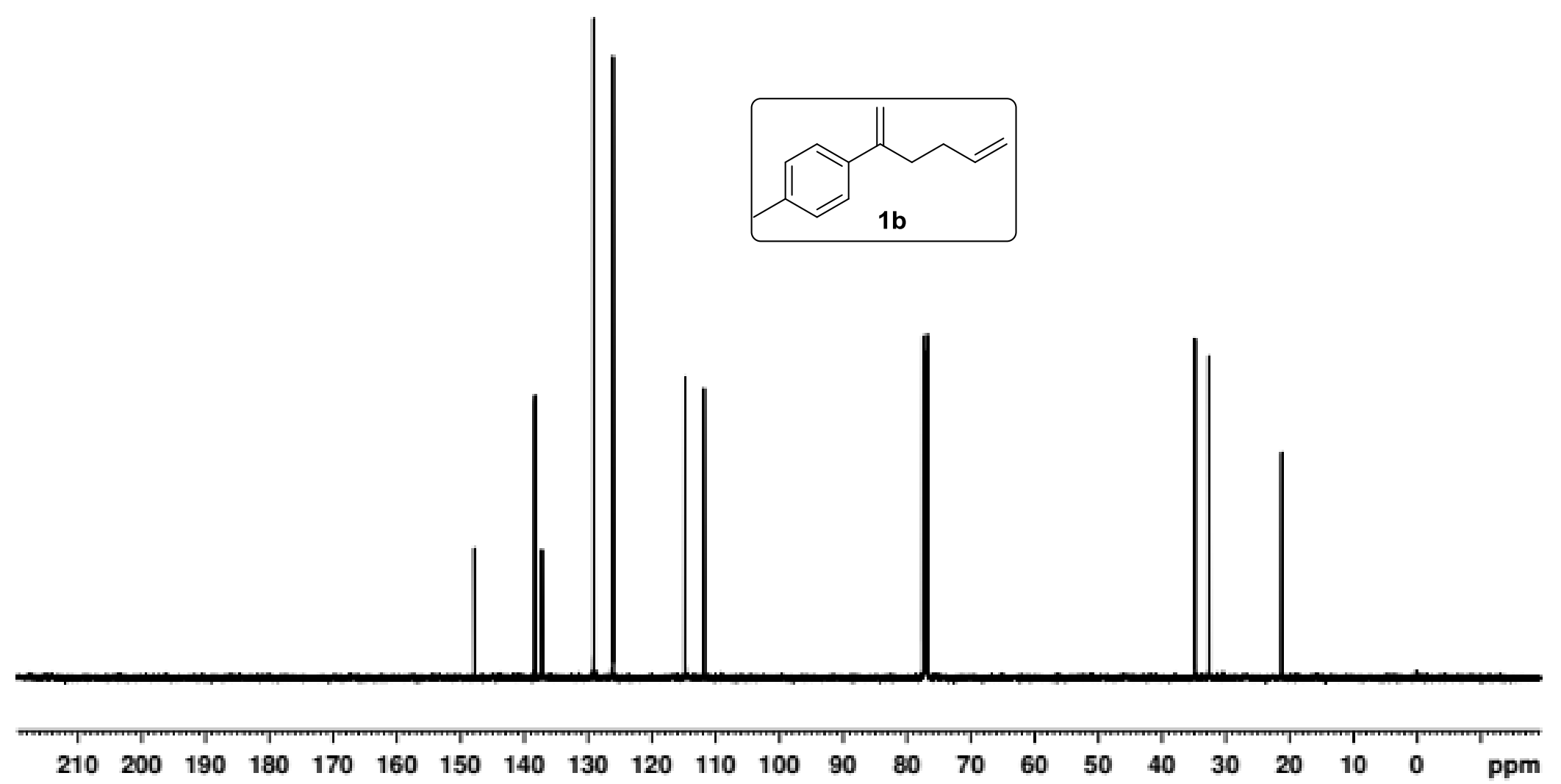


HY-X180122-3-CDC13-HNMR
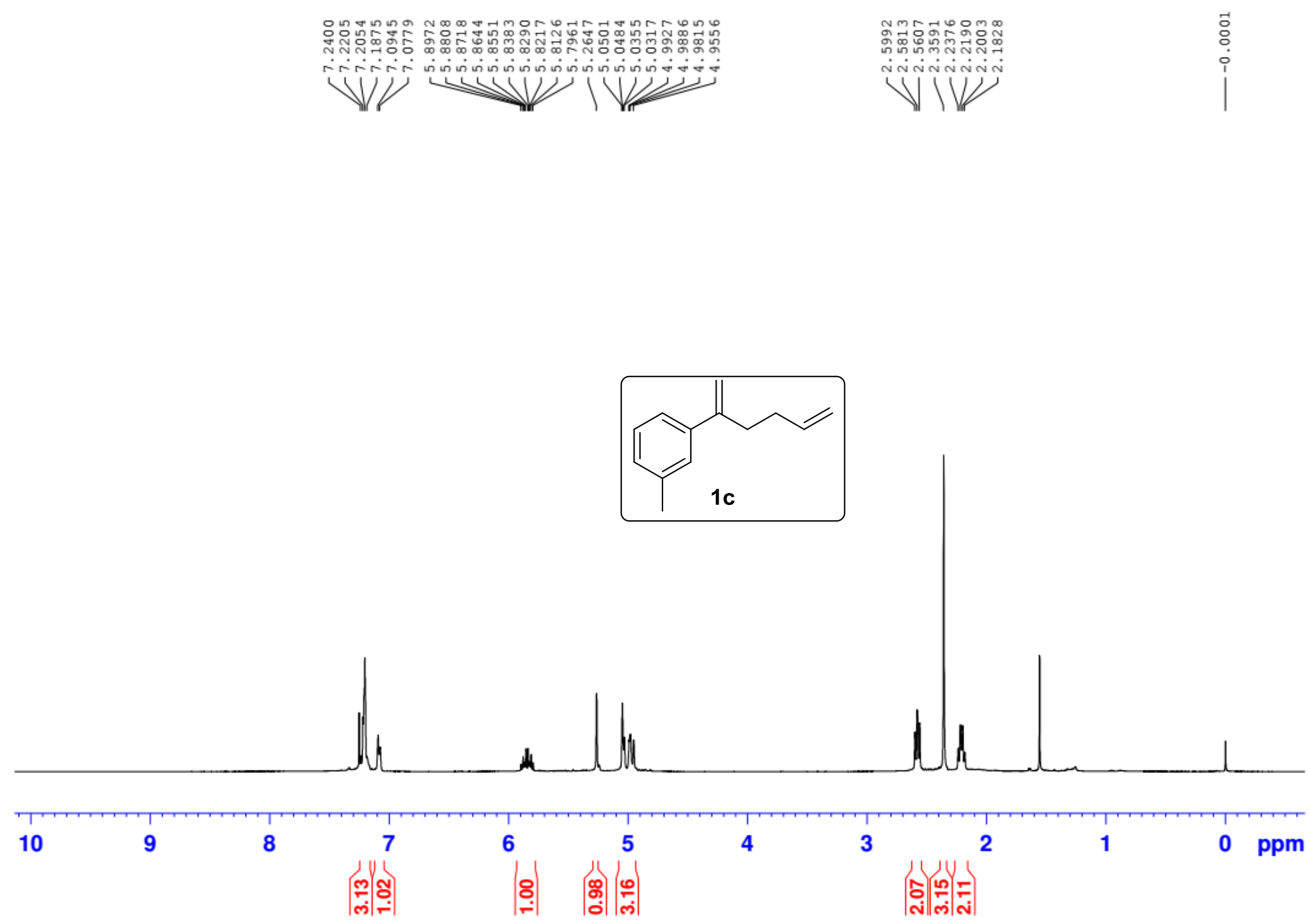
HY-X180122-3-CHC13-CNMR
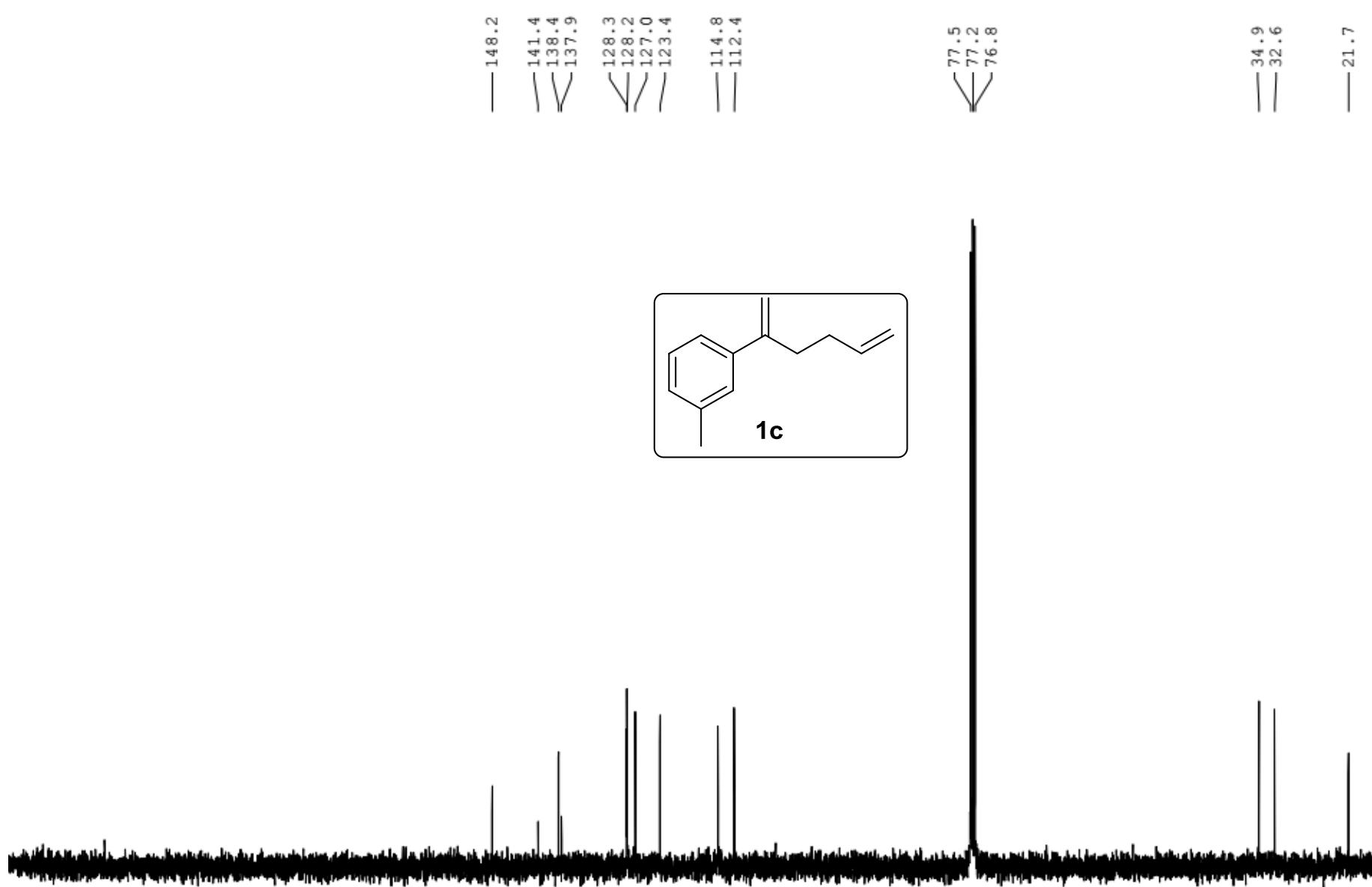

$\begin{array}{llllllllllllllllllllllll}210 & 200 & 190 & 180 & 170 & 160 & 150 & 140 & 130 & 120 & 110 & 100 & 90 & 80 & 70 & 60 & 50 & 40 & 30 & 20 & 10 & 0 & \text { ppm }\end{array}$ 
HY-X180401-1-CDC13-HNMR
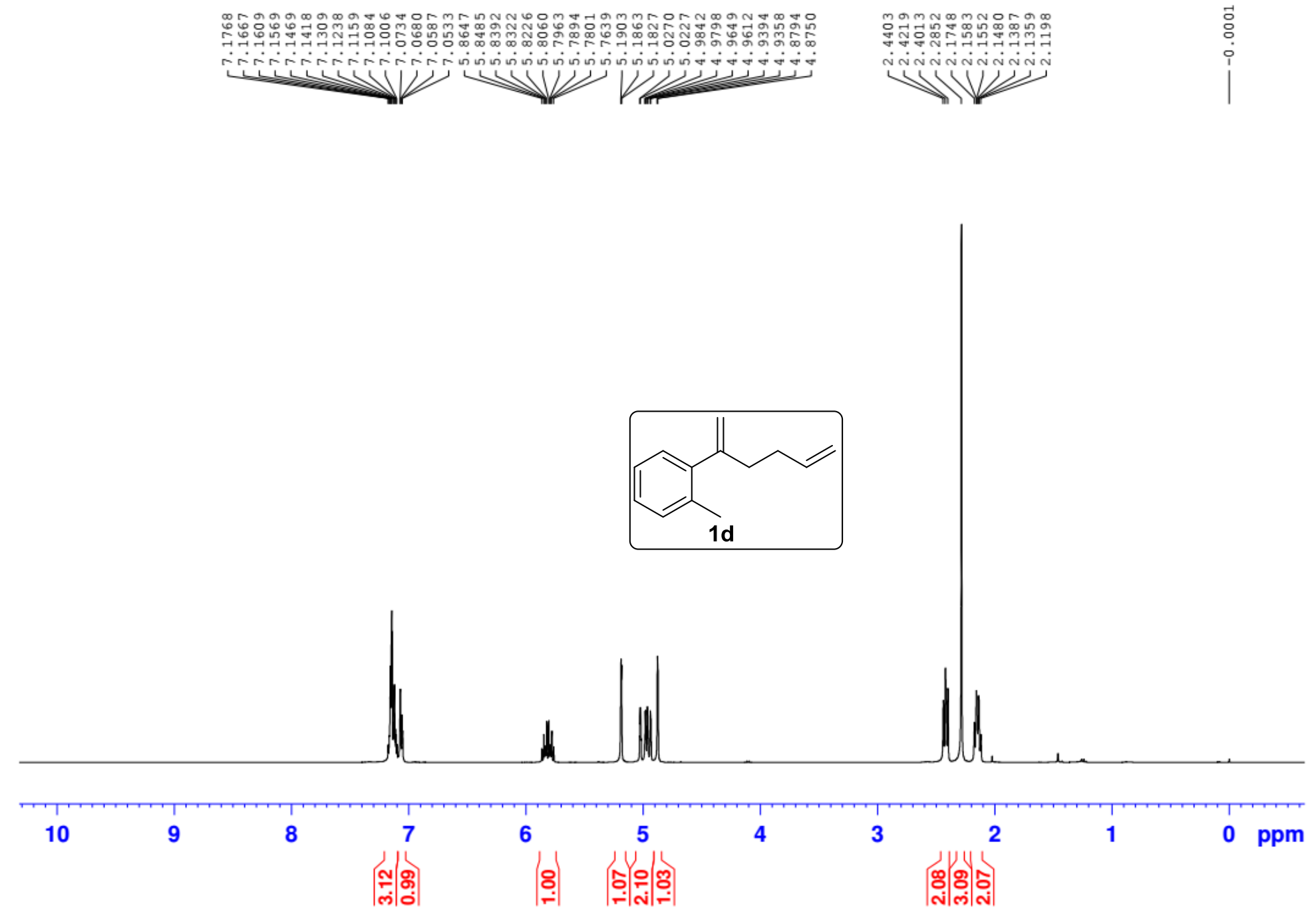
HY-X180401-1-CDC13-CNMR
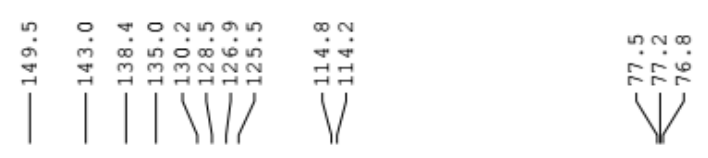

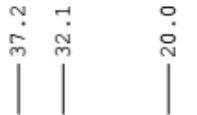

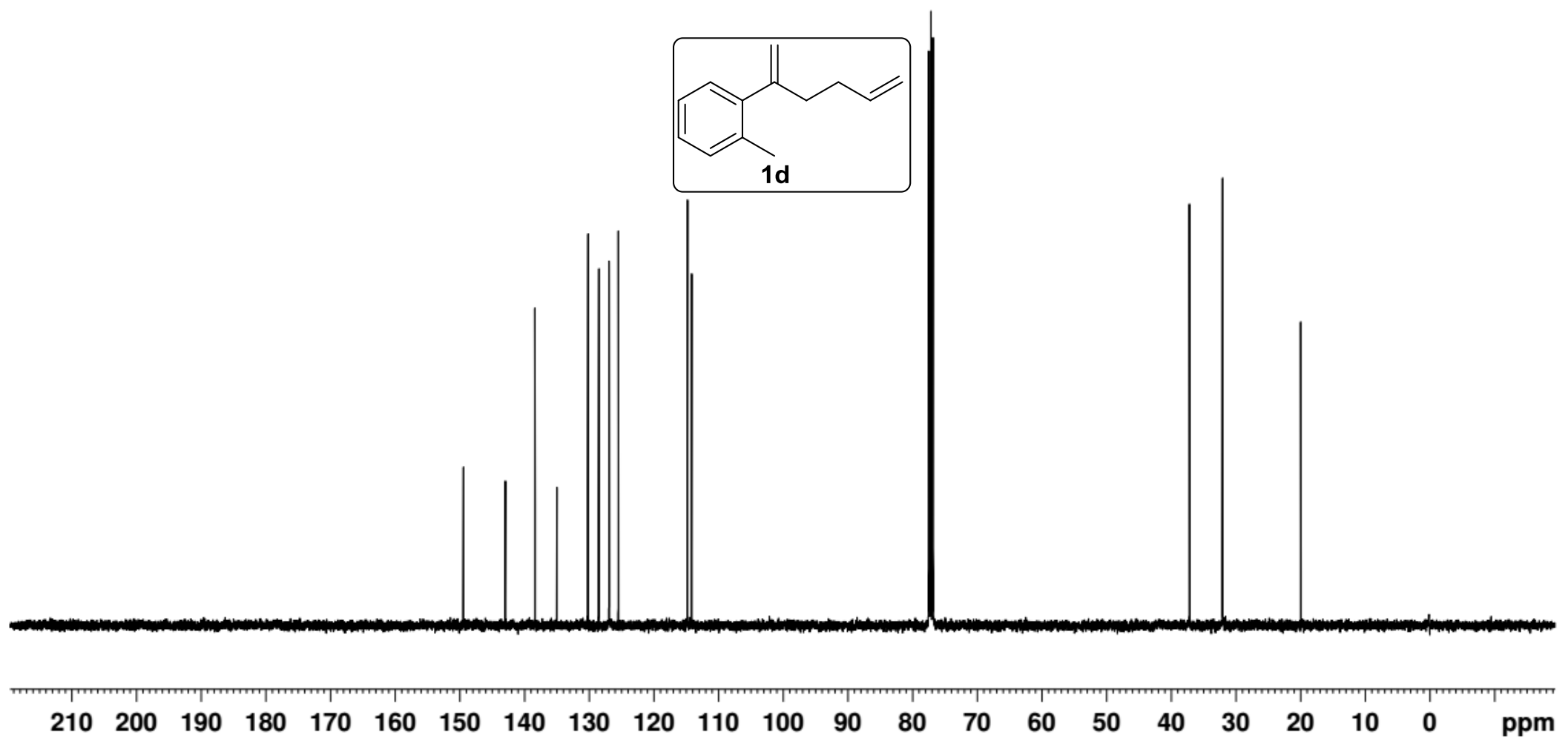


HY-X180124-5-CDC13-HNMR
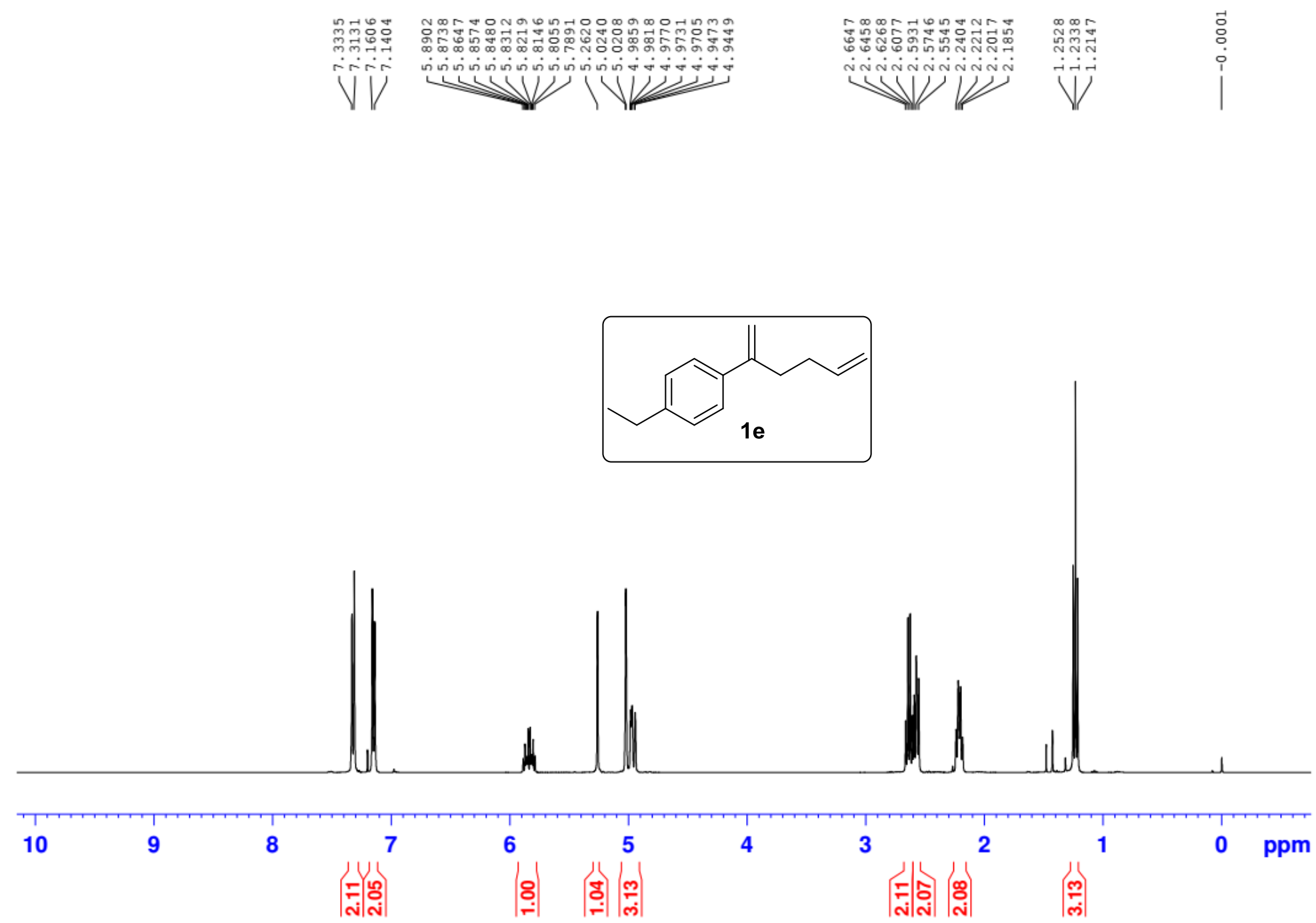
HY-X180124-5-CDCl3-CNMR
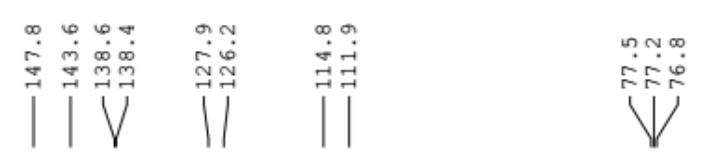

||$^{\infty}$

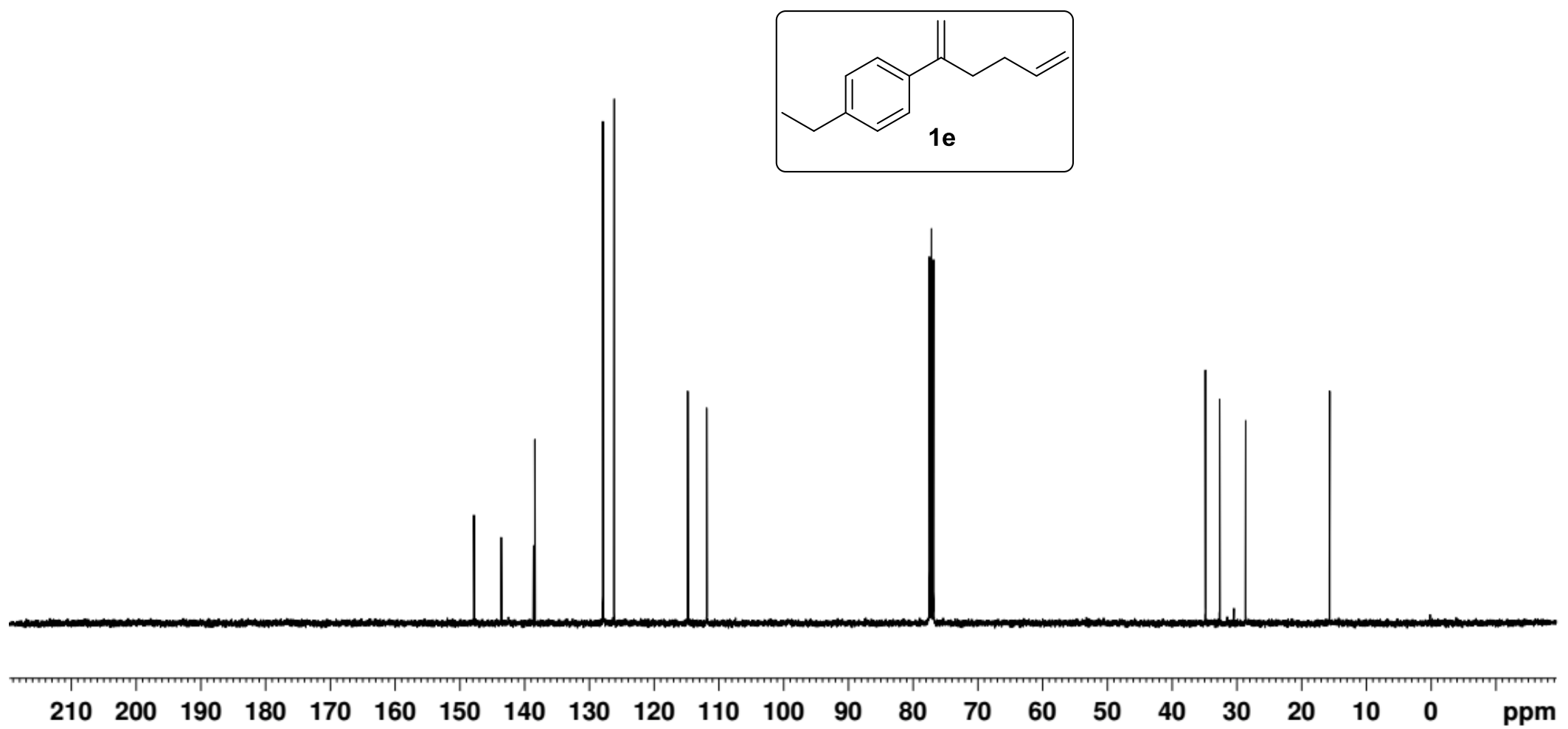


HY-X180124-3-CDC13-HNMR
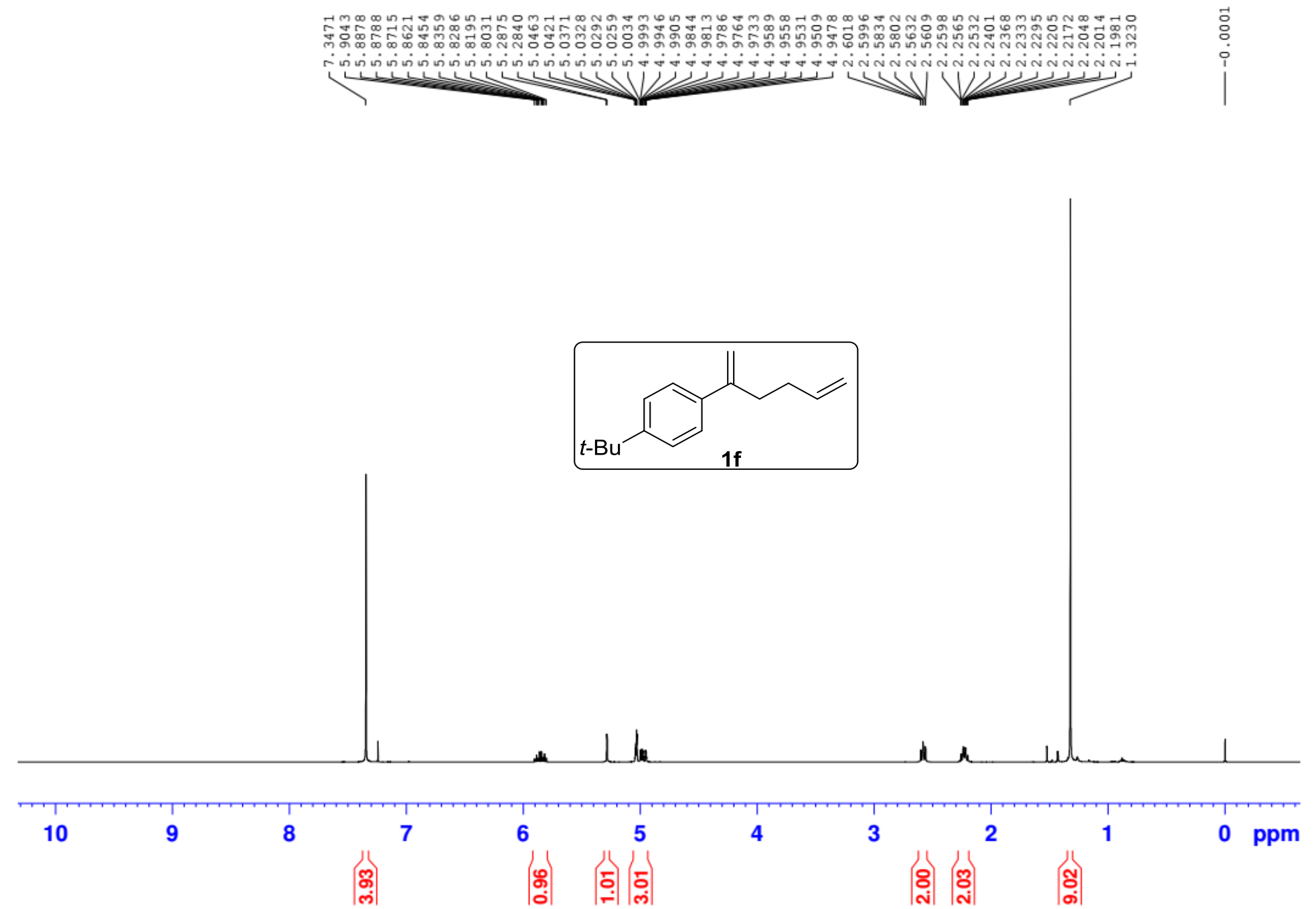
HY-X180124-3-CDC13-CNMR
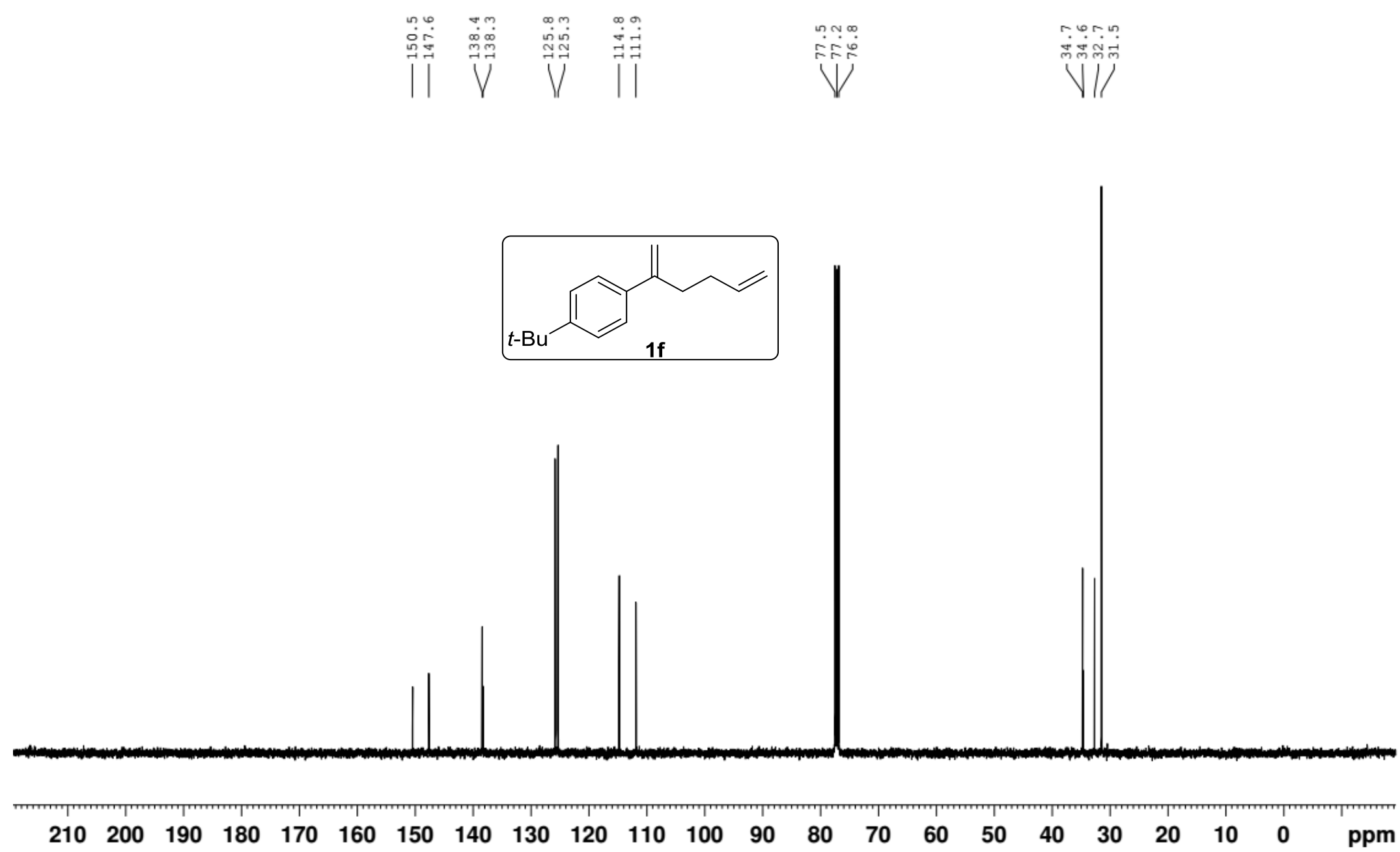


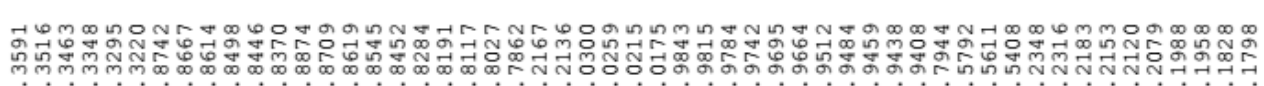

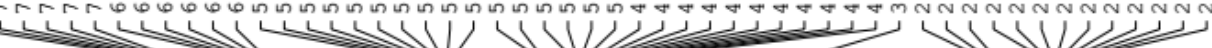

$\rightarrow$
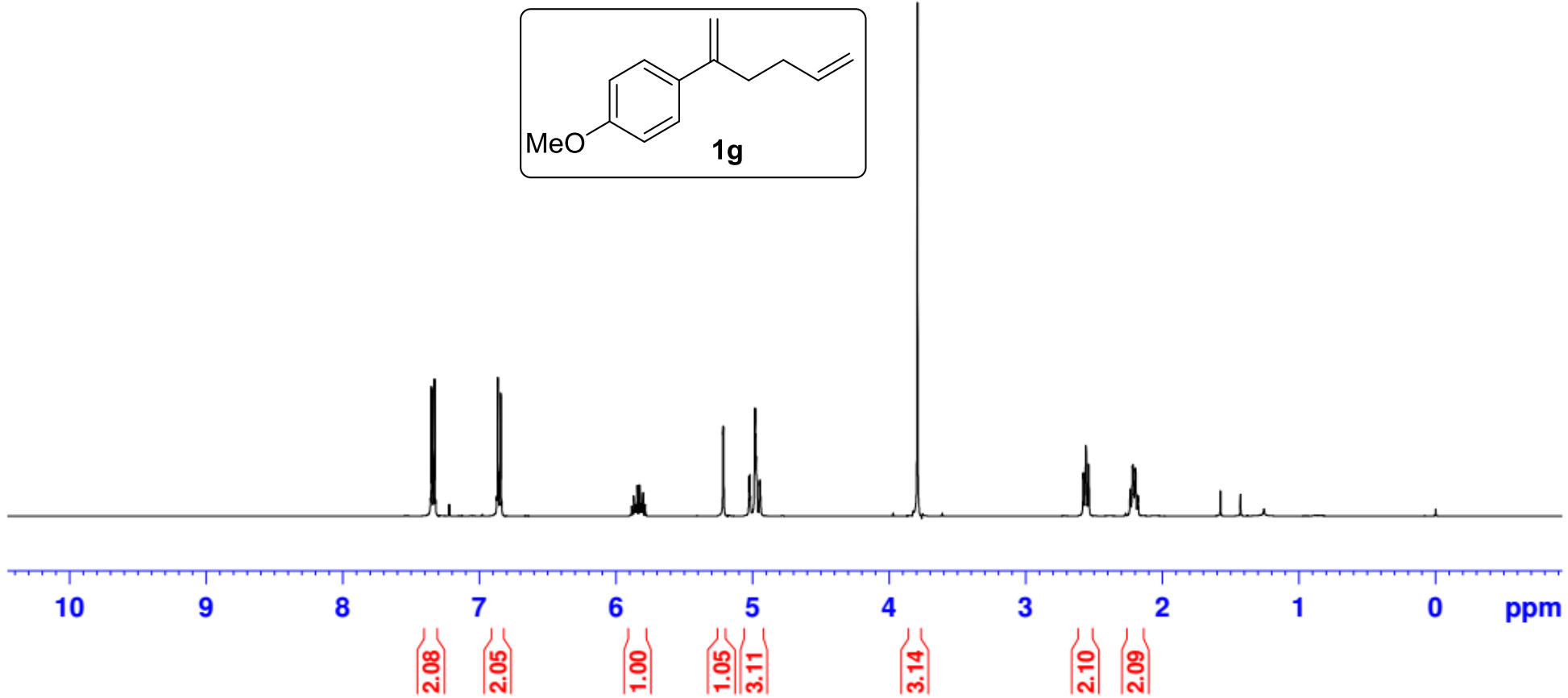


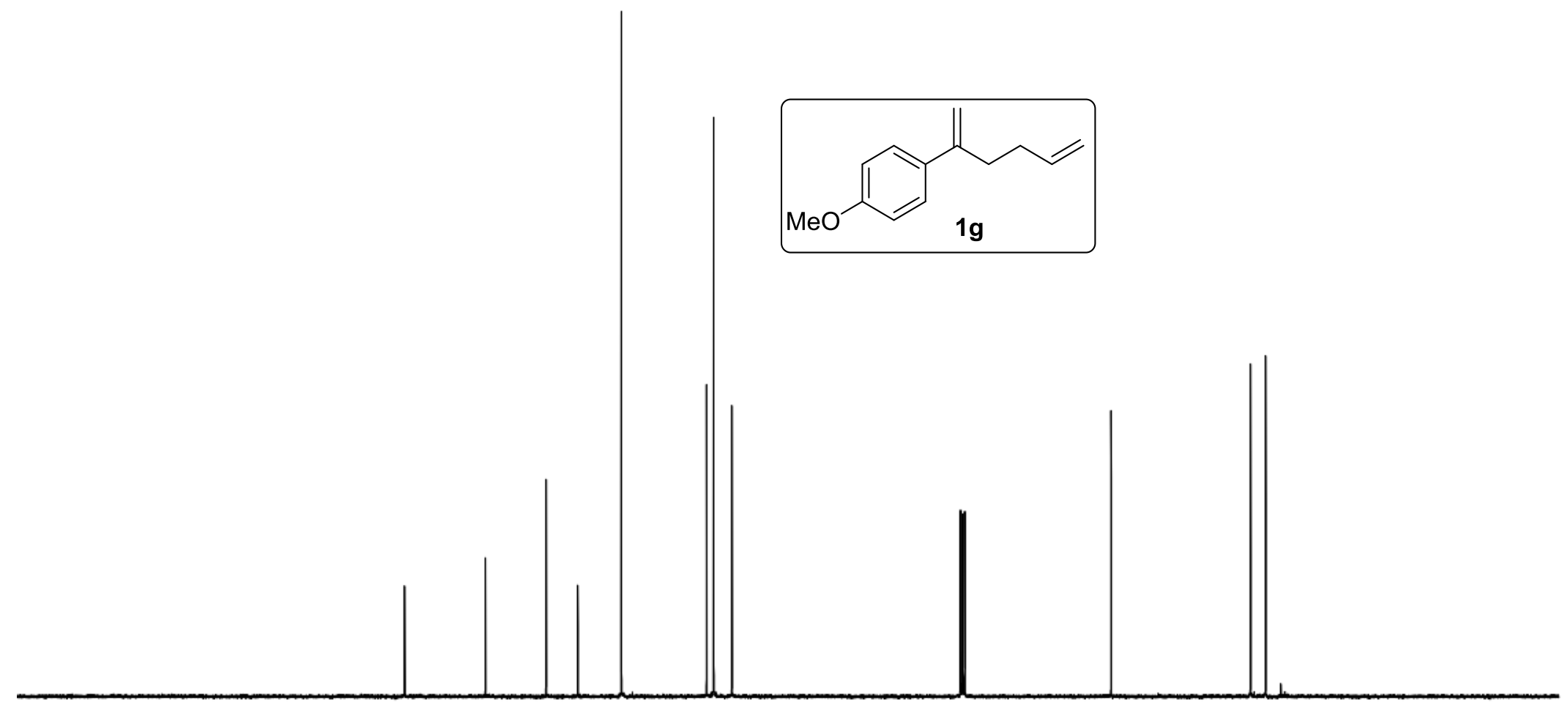

$\begin{array}{lllllllllllllllllllllll}210 & 200 & 190 & 180 & 170 & 160 & 150 & 140 & 130 & 120 & 110 & 100 & 90 & 80 & 70 & 60 & 50 & 40 & 30 & 20 & 10 & \text { ppm }\end{array}$ 
HY-X180424-4-CDC13-HNMR
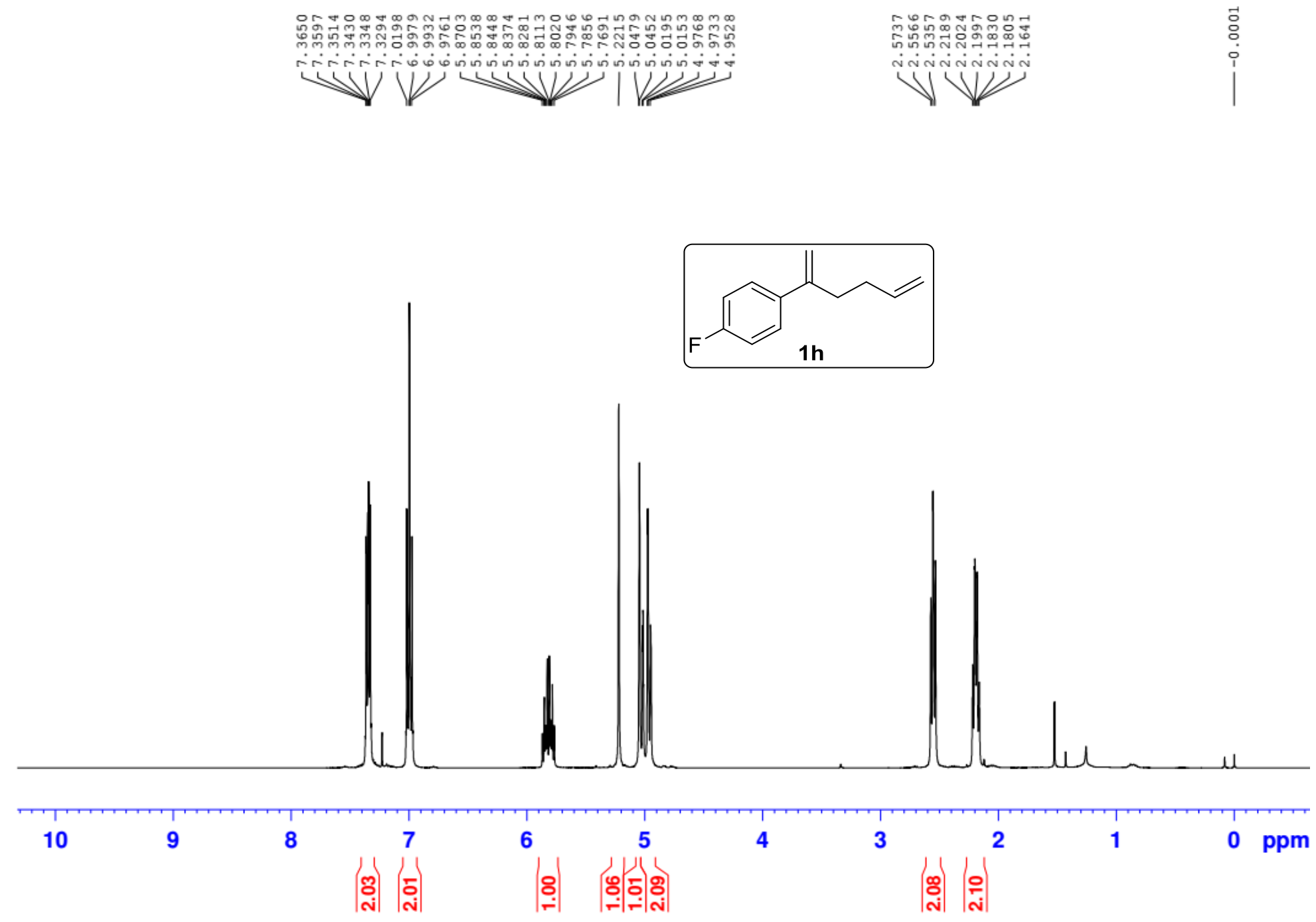
HY-X180424-4-CDC13-CNMR
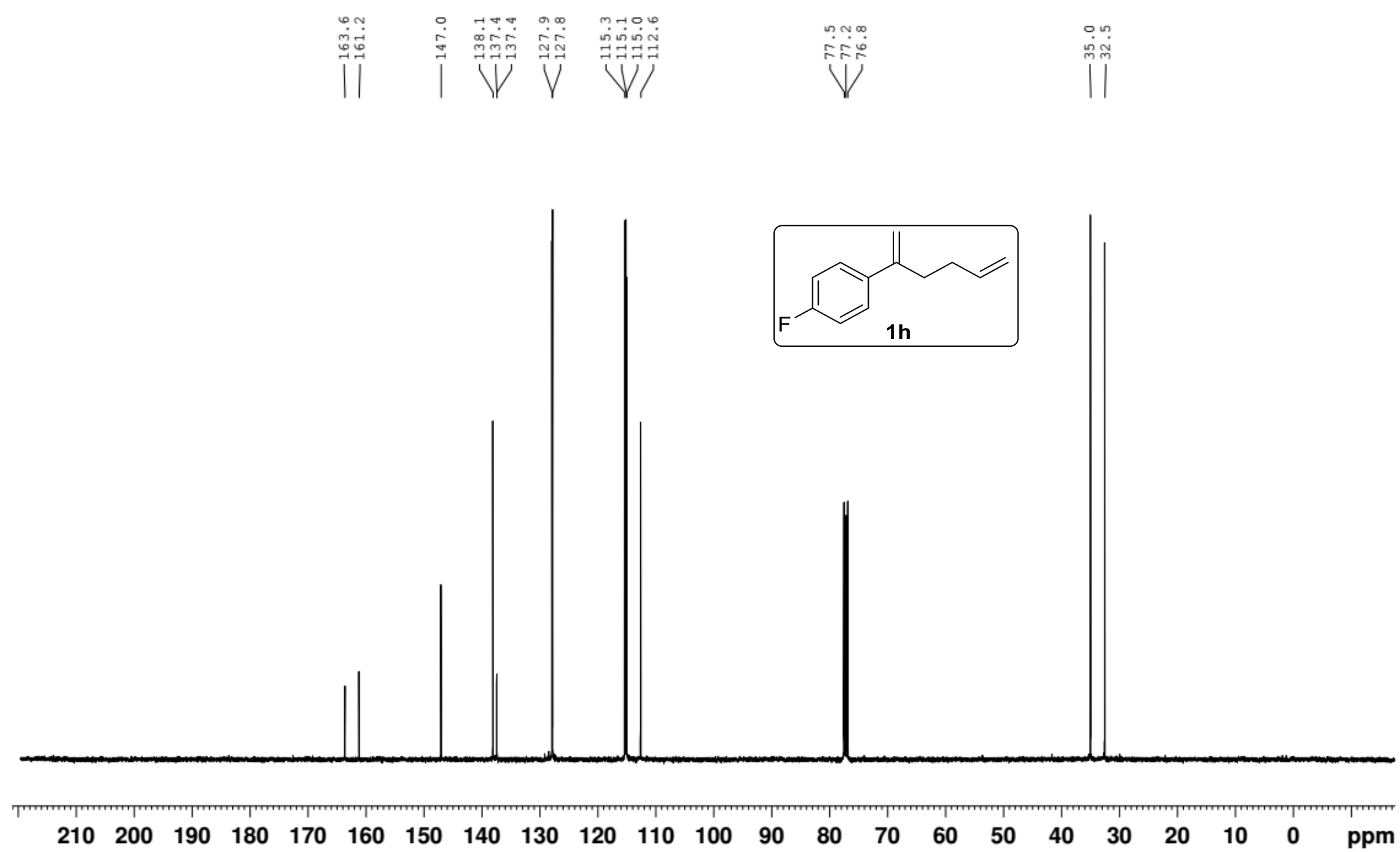
HY-X180424-4-CDC13-FNMR

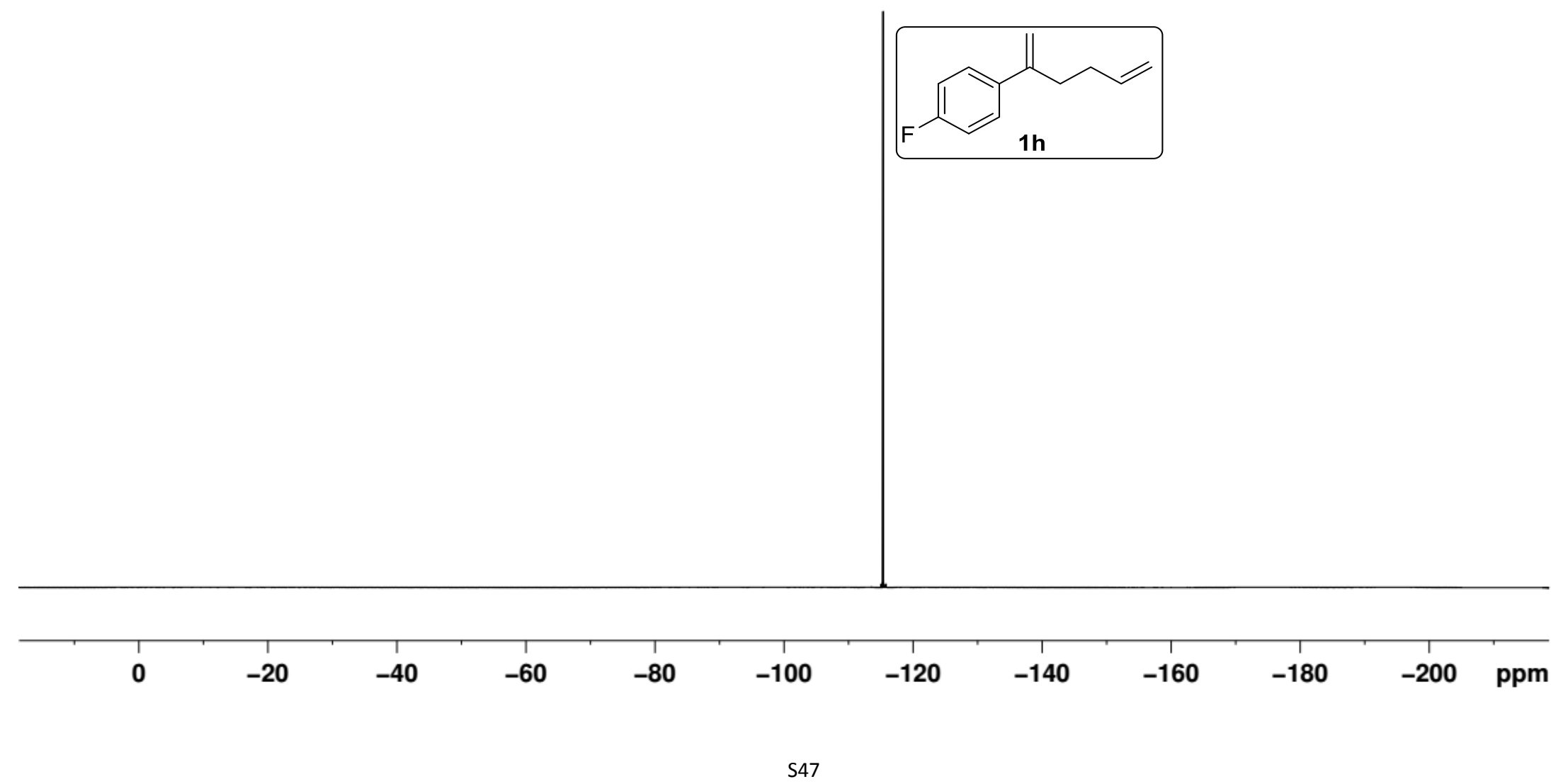




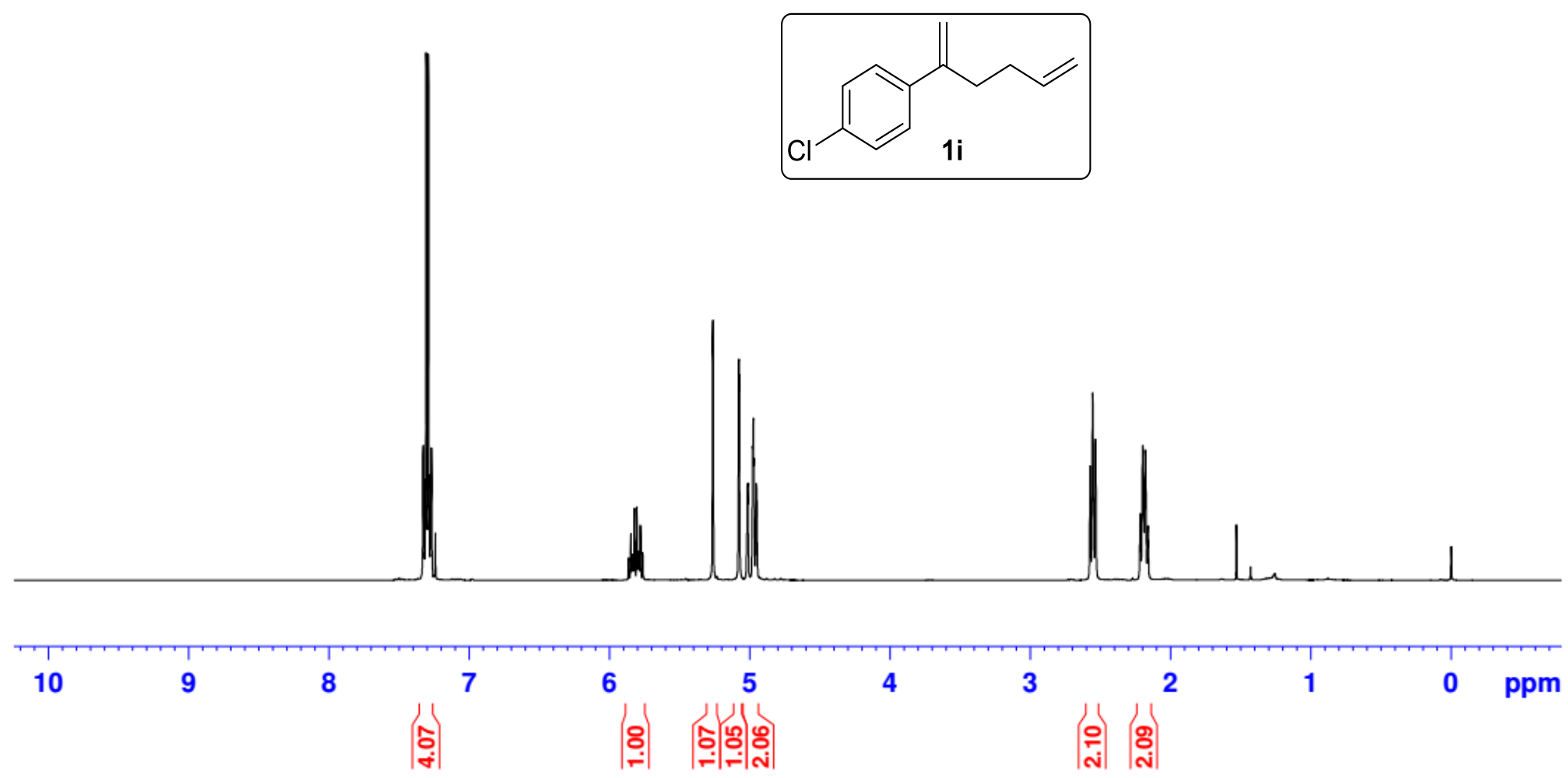


HY-X180124-1-CDC13-CNMR

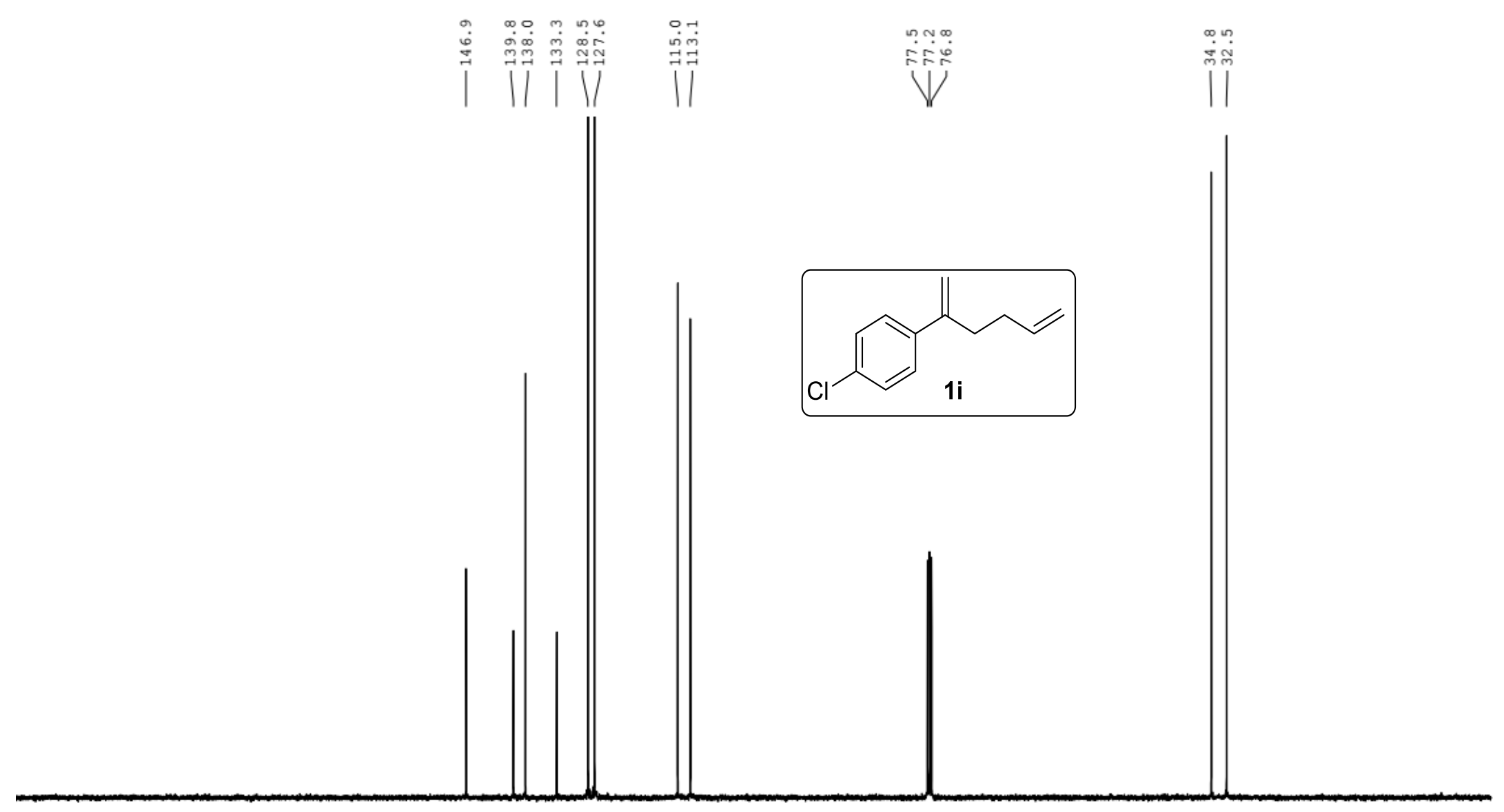

$\begin{array}{lllllllllllllllllllllll}210 & 200 & 190 & 180 & 170 & 160 & 150 & 140 & 130 & 120 & 110 & 100 & 90 & 80 & 70 & 60 & 50 & 40 & 30 & 20 & 10 & \text { ppm }\end{array}$ 
HY-X180124-2-CDC13-HNMR

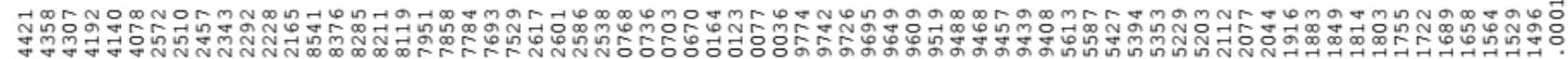

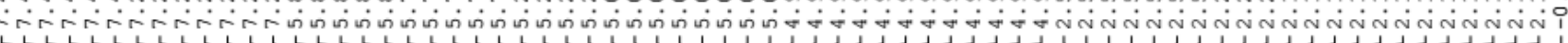

7

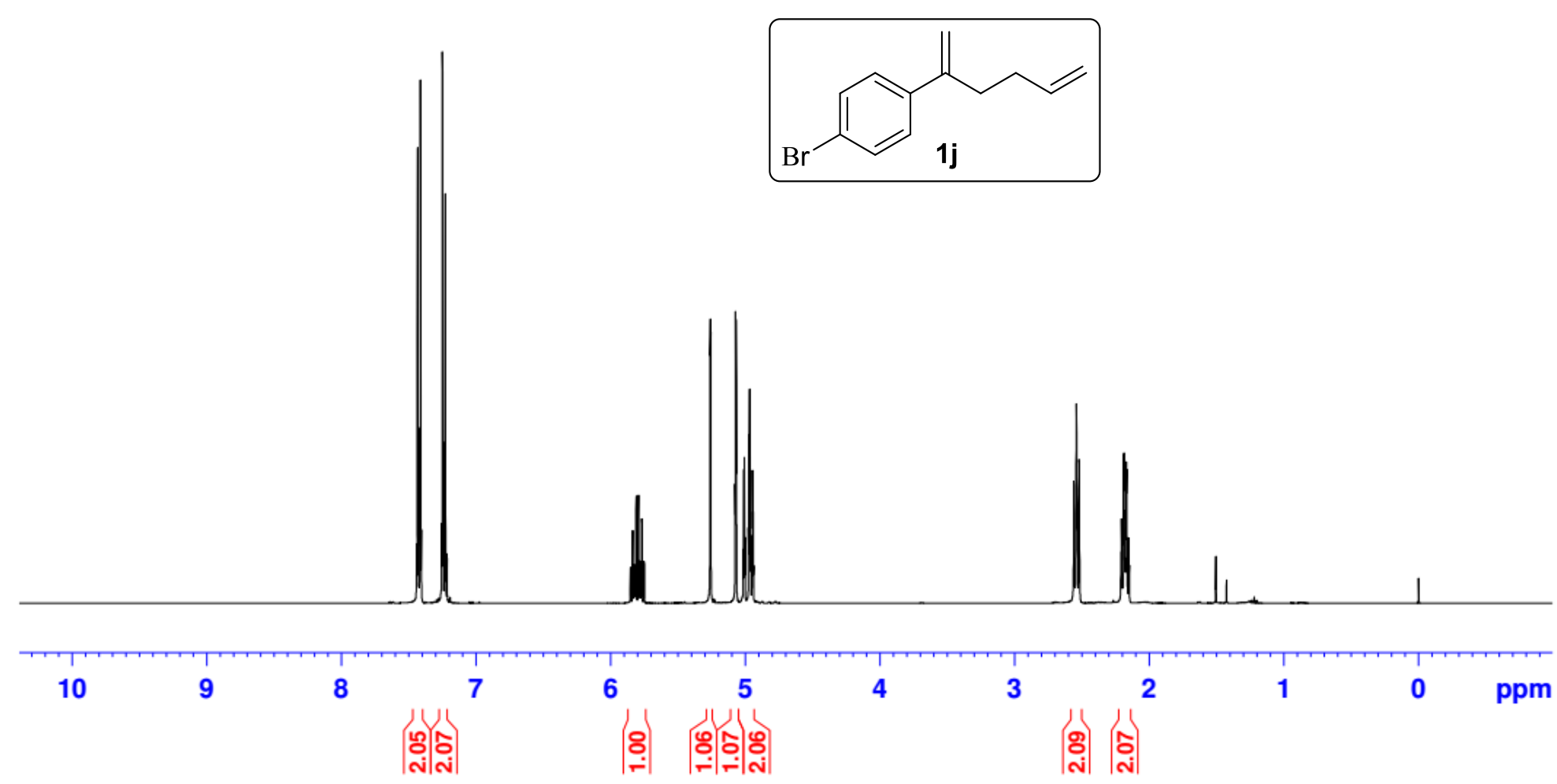




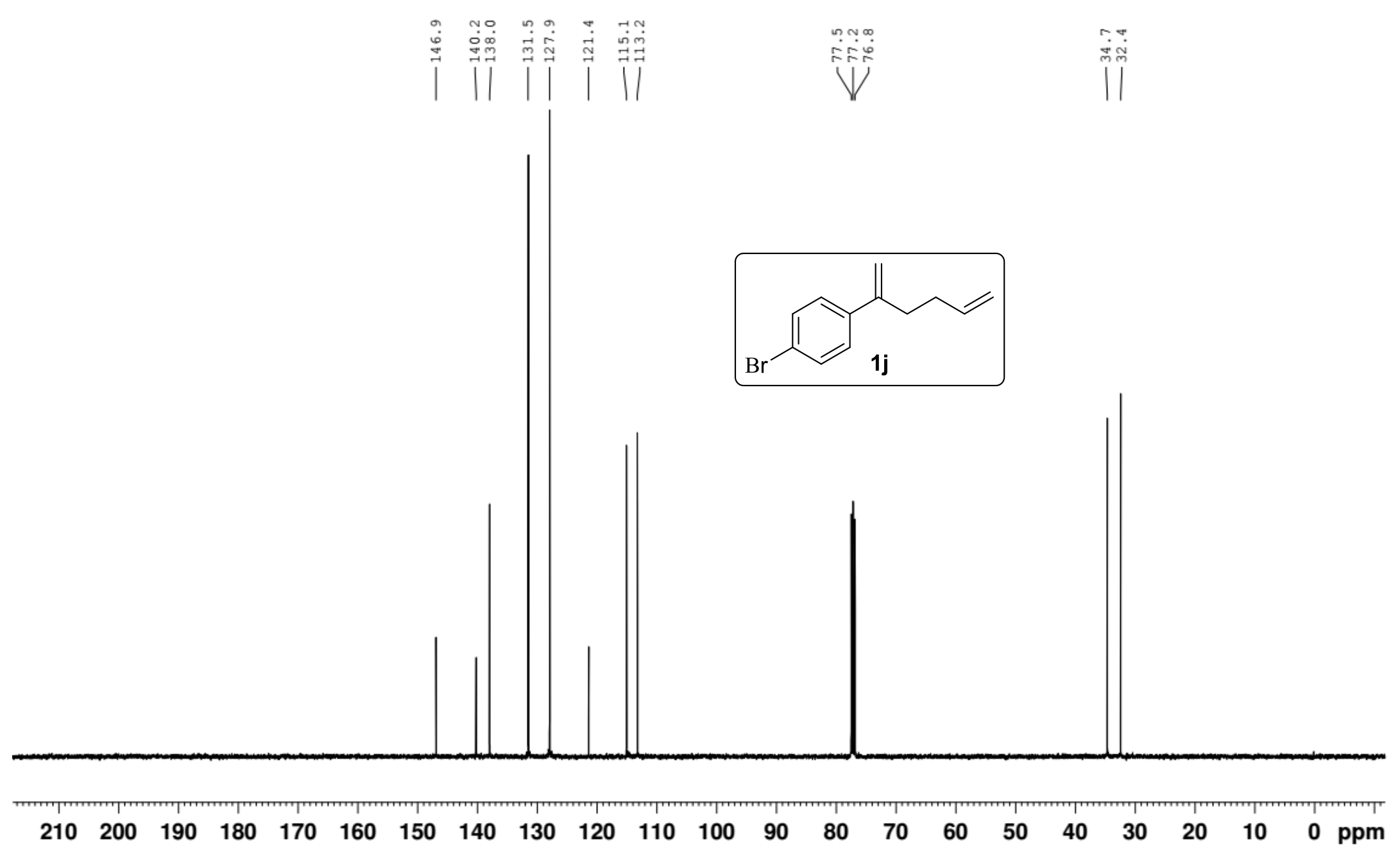




\section{HY-X180424-1-CDC13-HNMR}

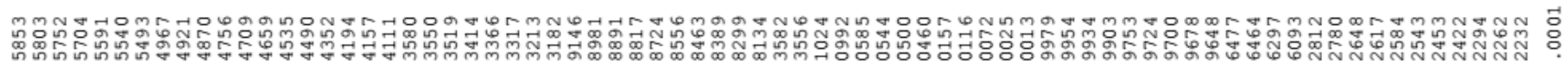

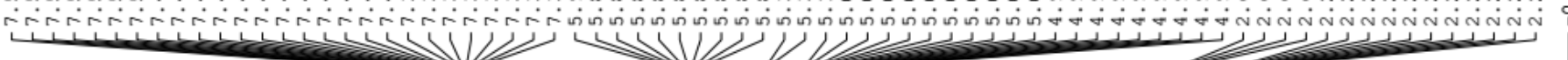
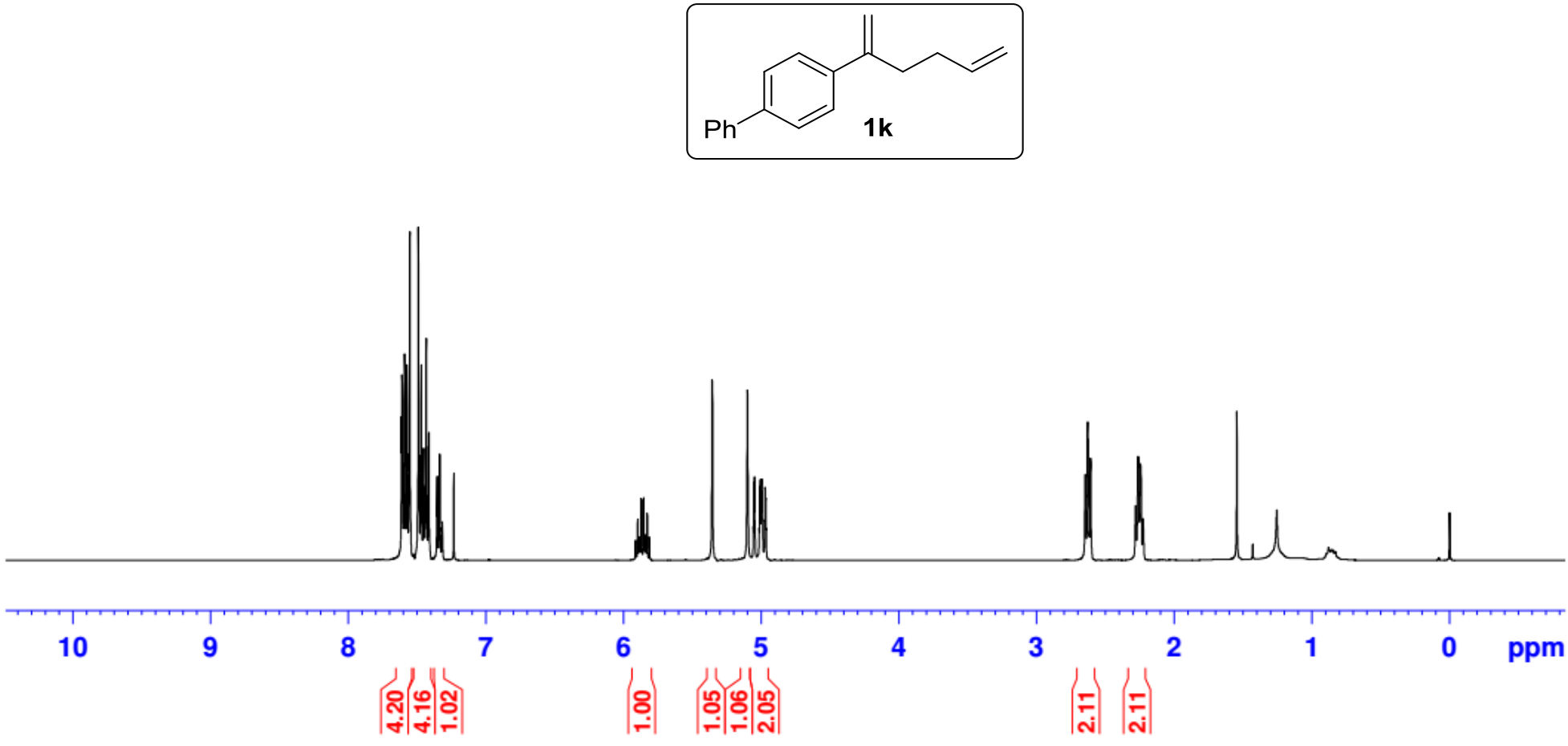
HY-X180424-1-CDC13-CNMR
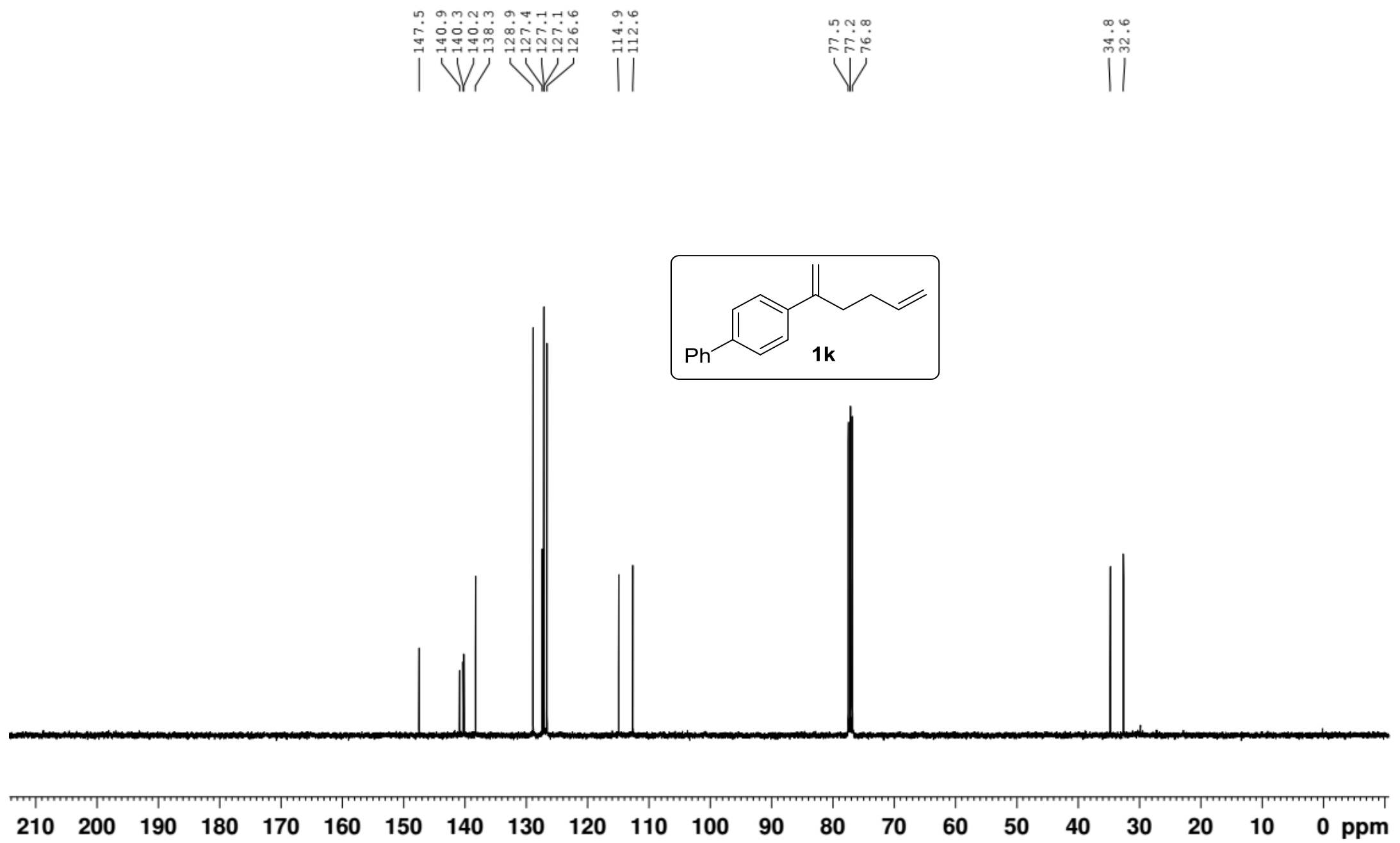

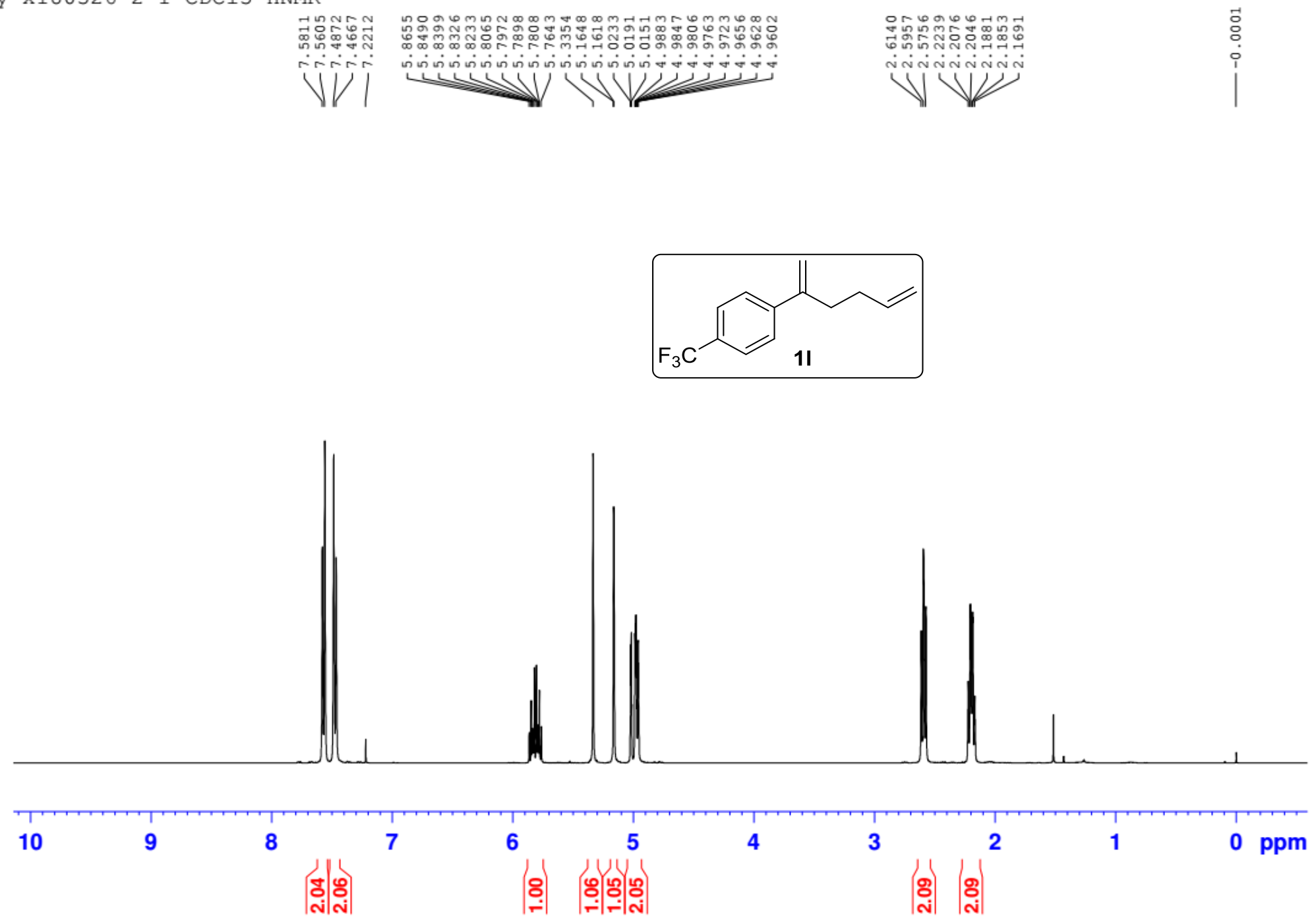


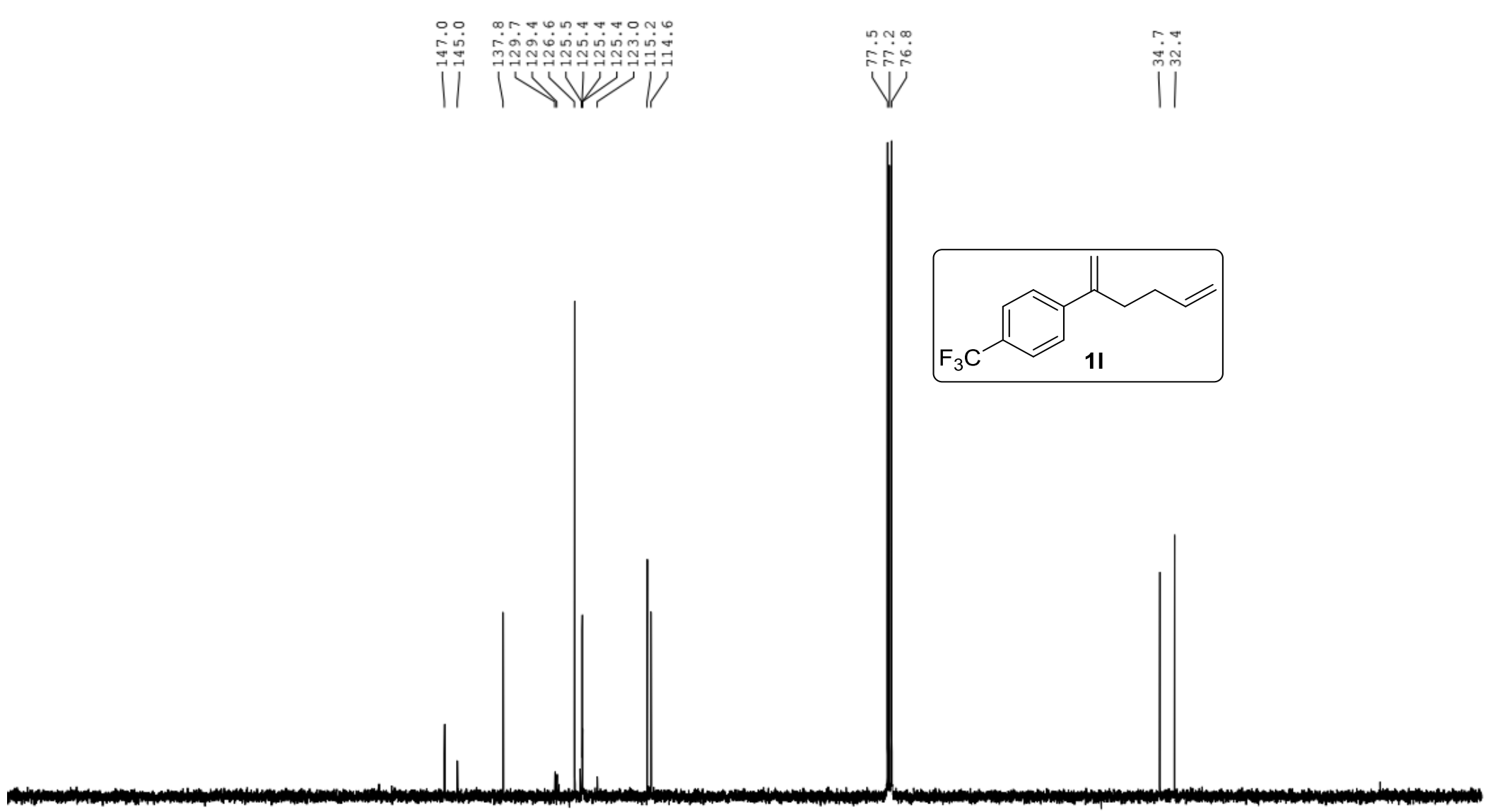


HY-X180326-2-1-CDC13-FNMR

|?
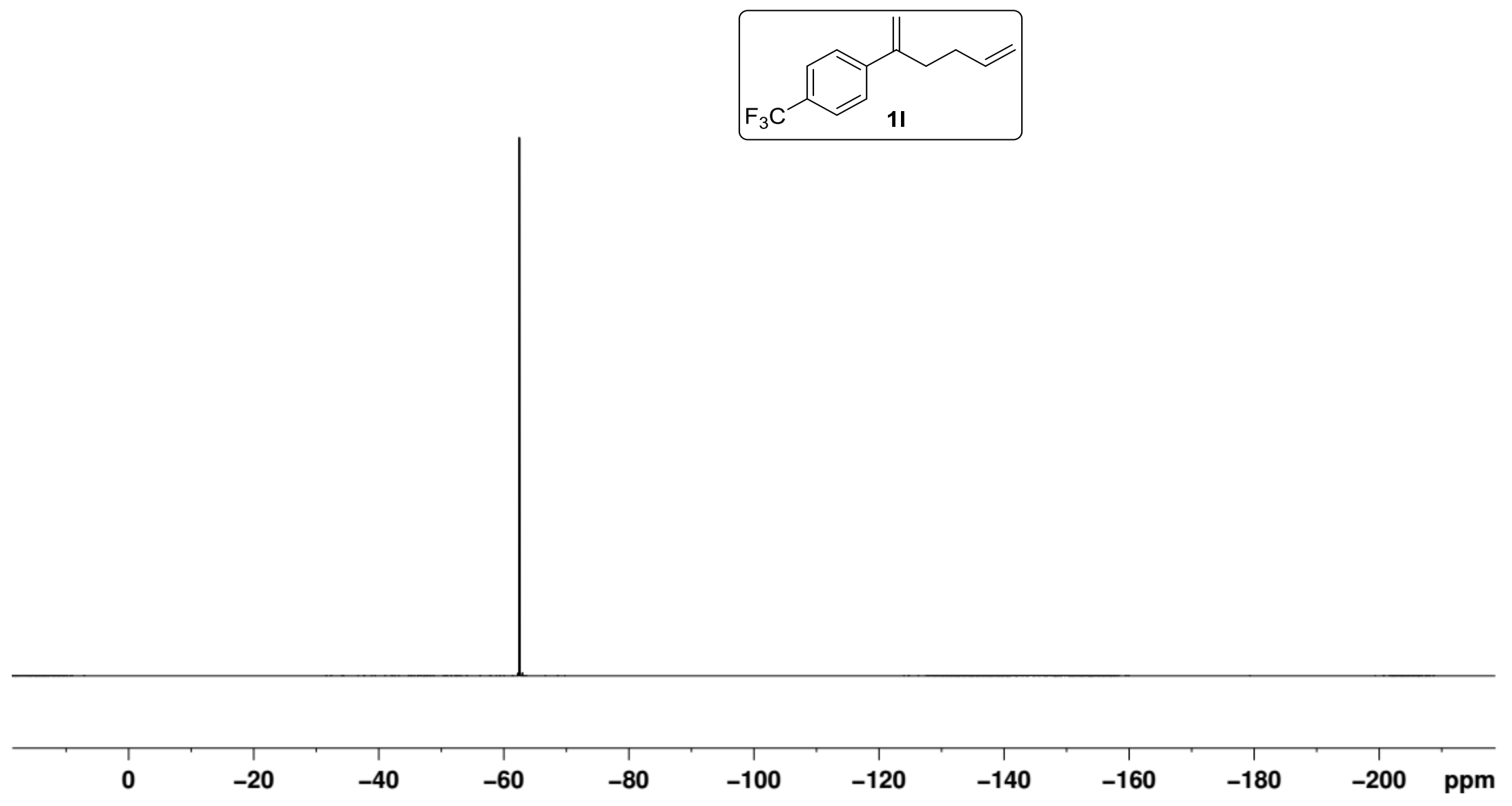
HY-X180321-1-CDC13-HNMR
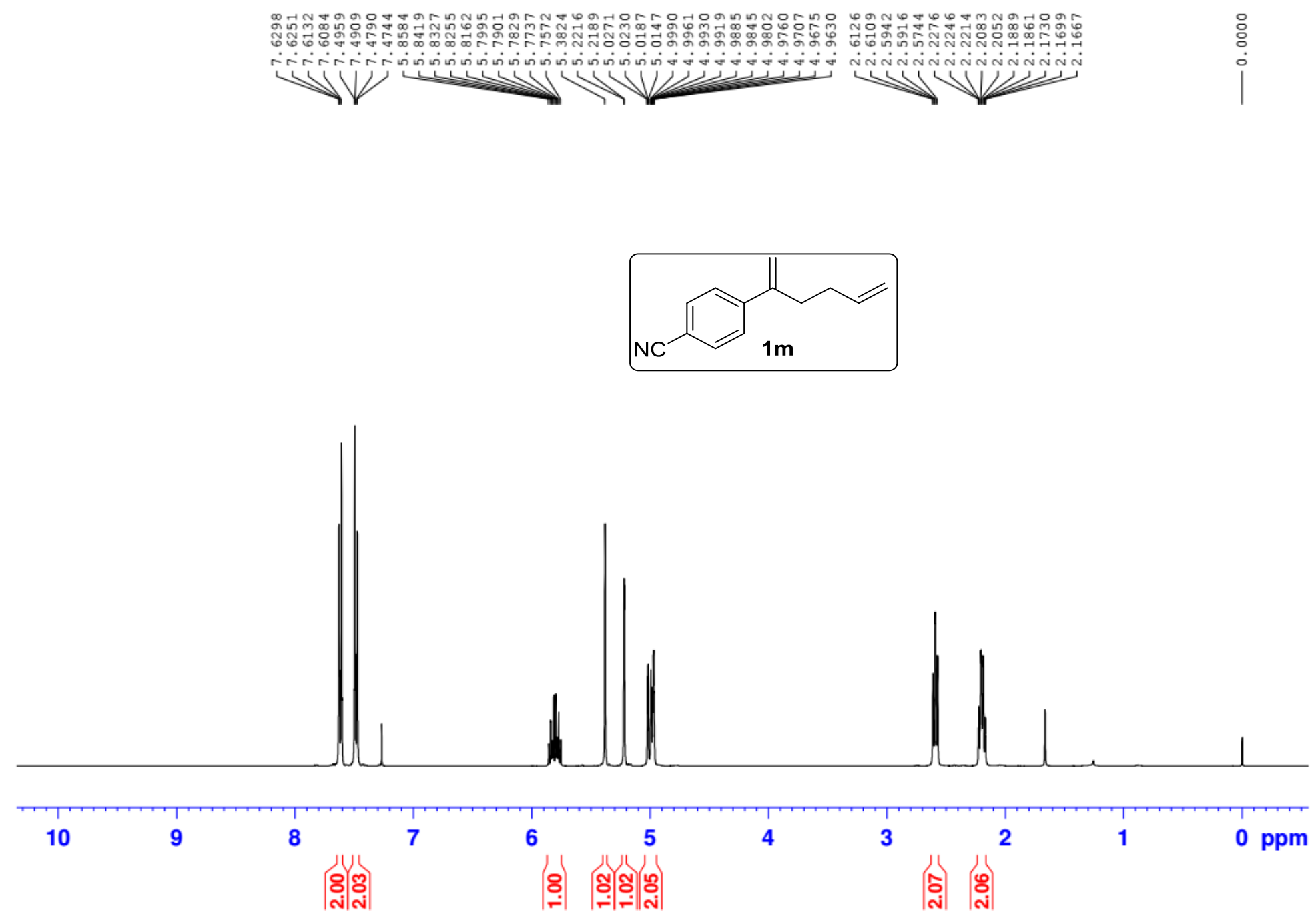
HY-X180321-1-CDC13-CNMR

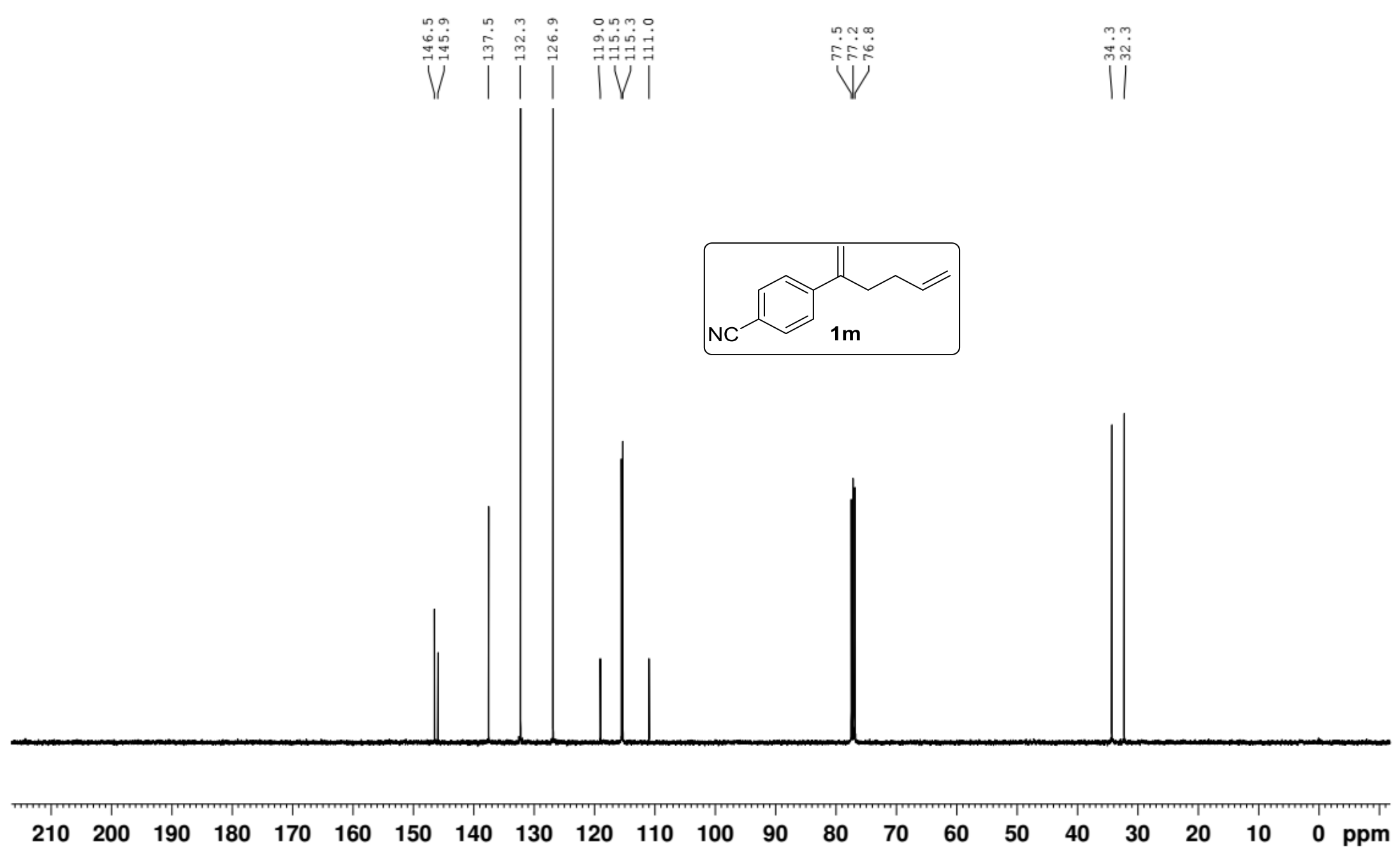


HY-X180408-1-CDC13-HNMR

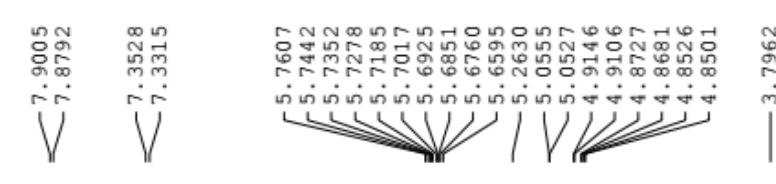

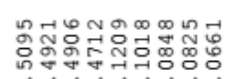

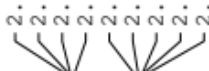
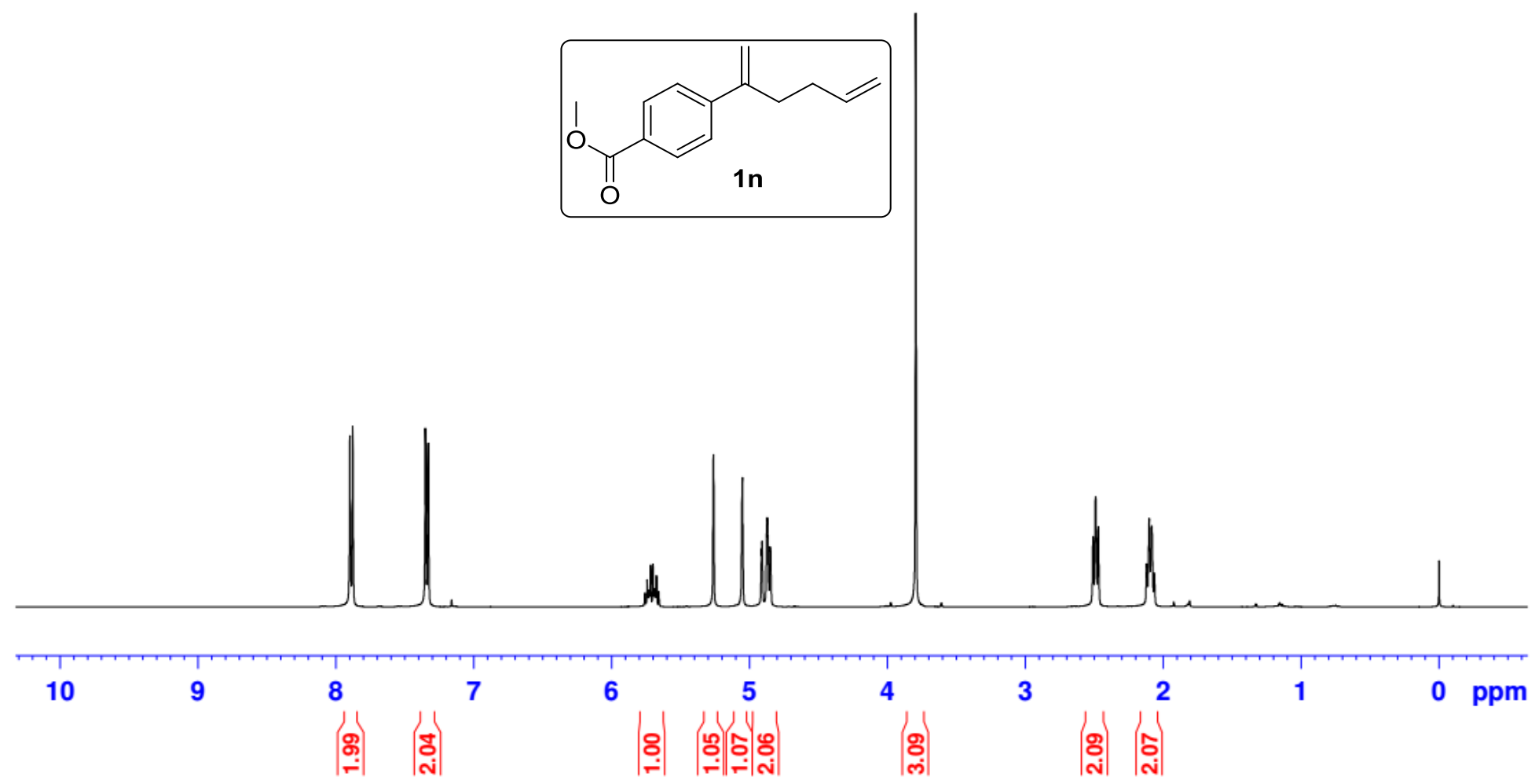
HY-X1800408-1-CDC13-CNMR

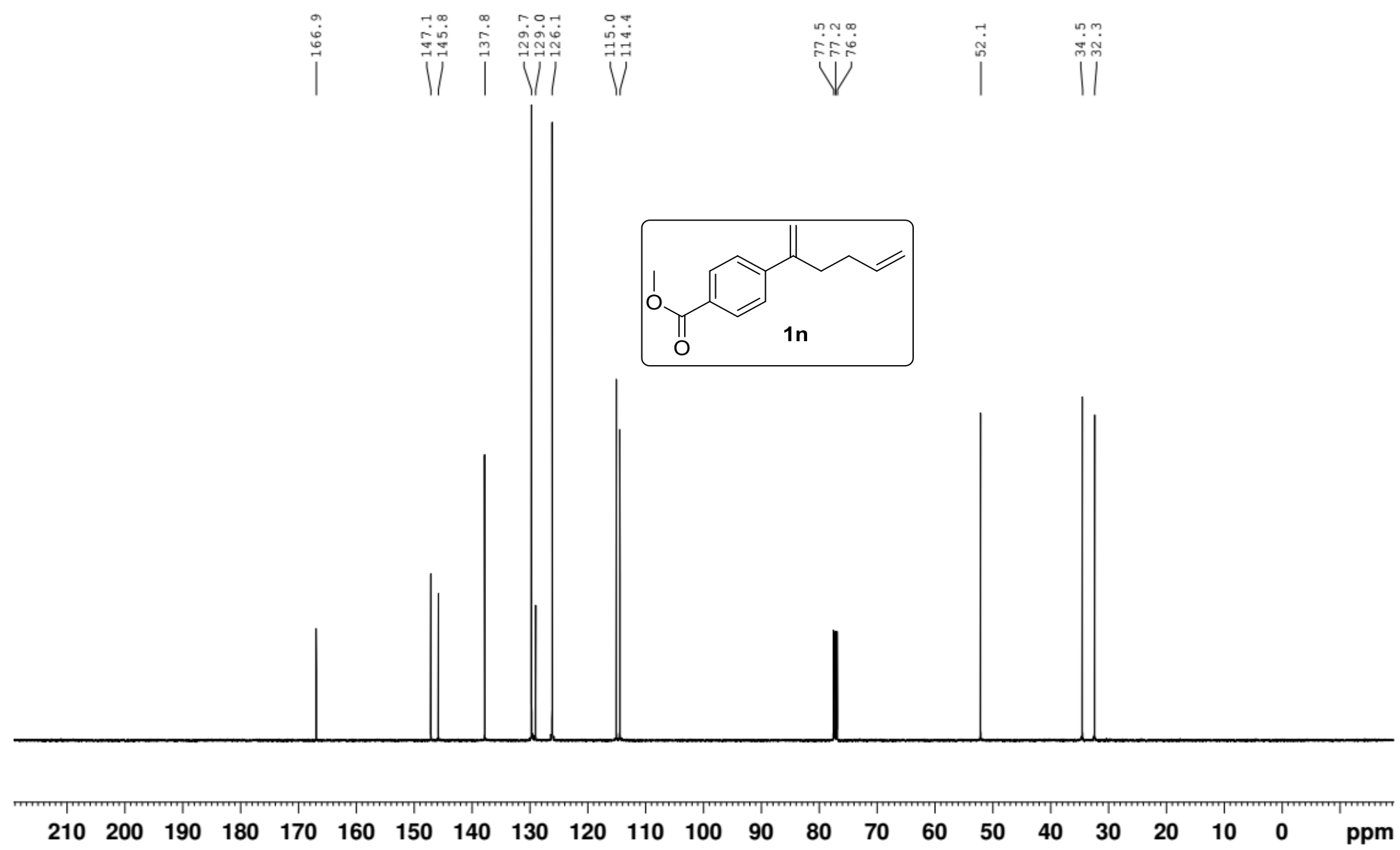


HY-X180226-1-CDC13-HNMR

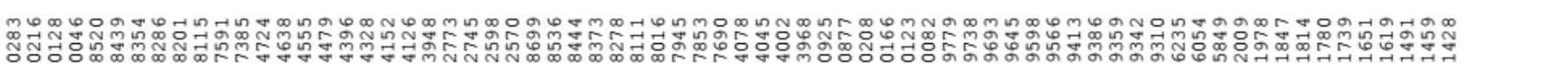

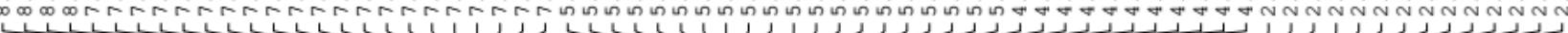
an
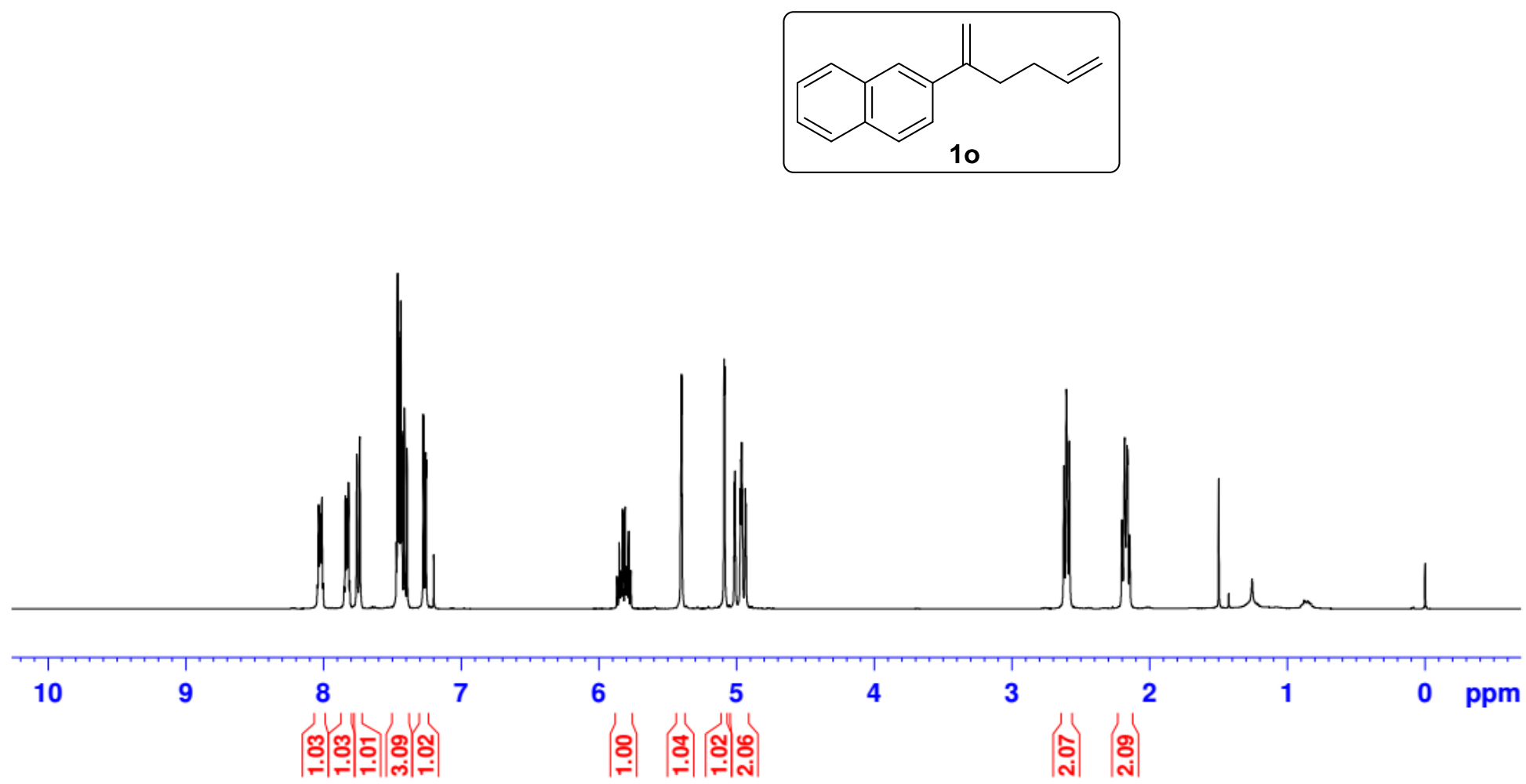
HY-X180226-1-CDC13-CNMR
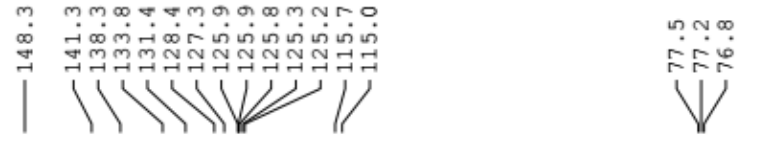

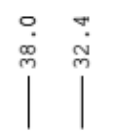
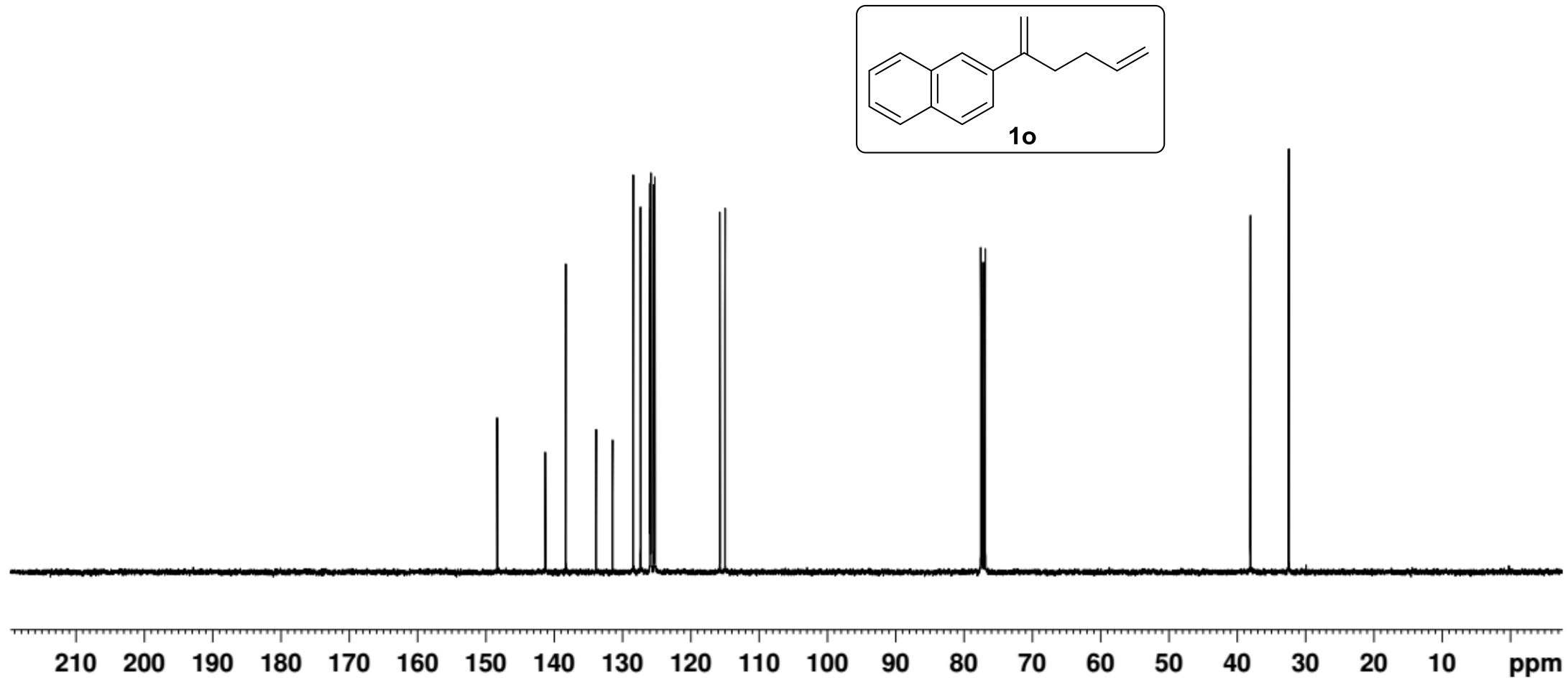
HY-X180424-1-CDC13-HNMR
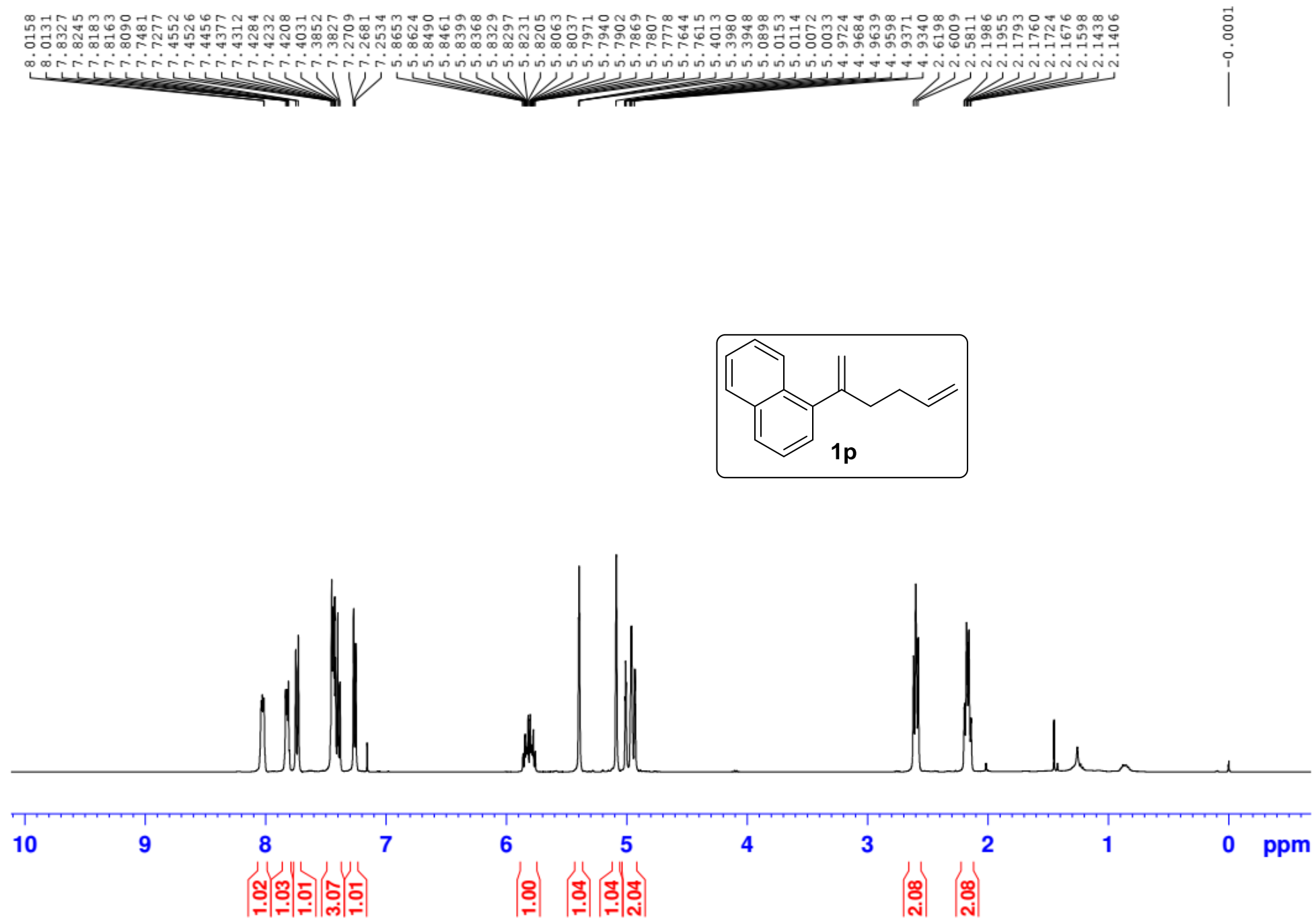

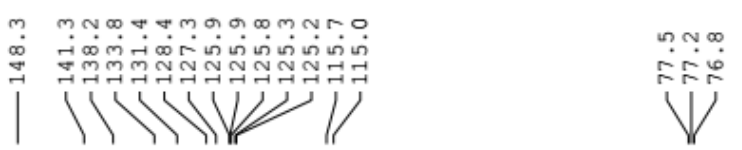

$\stackrel{\circ}{\infty}$

$\left.\right|^{m}$

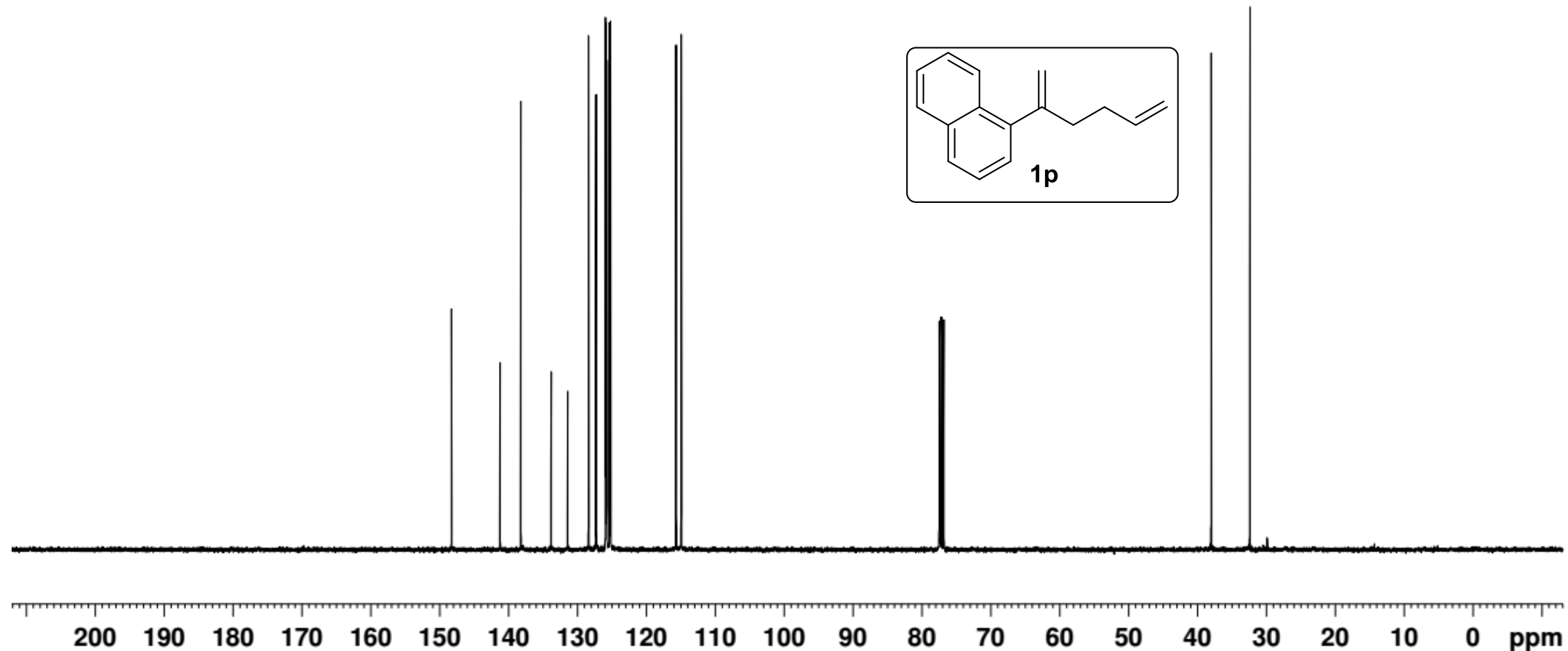


HY-X180430-1-CDC13-HNMR
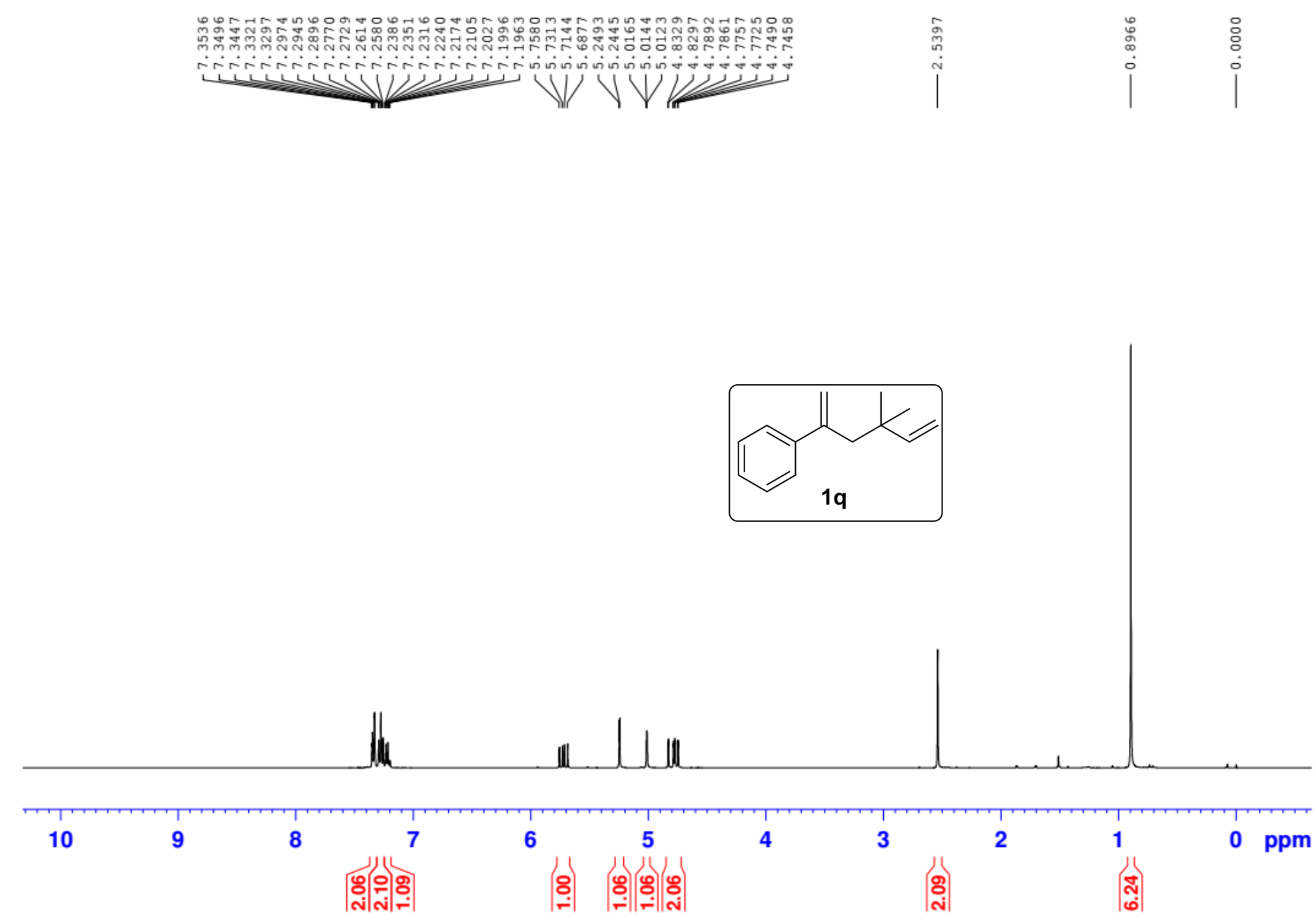
HY-X180430-1-CDC13-CNMR

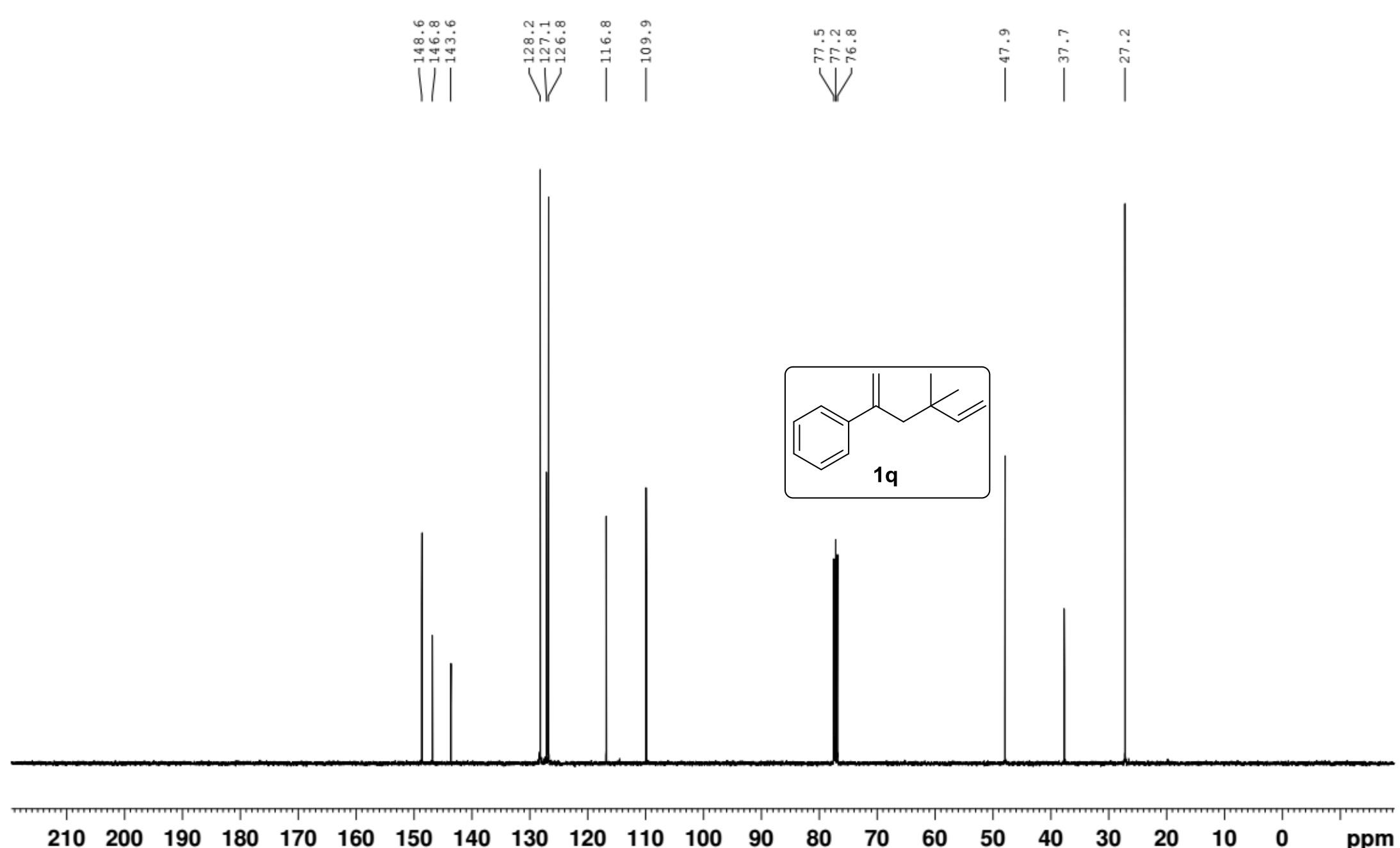




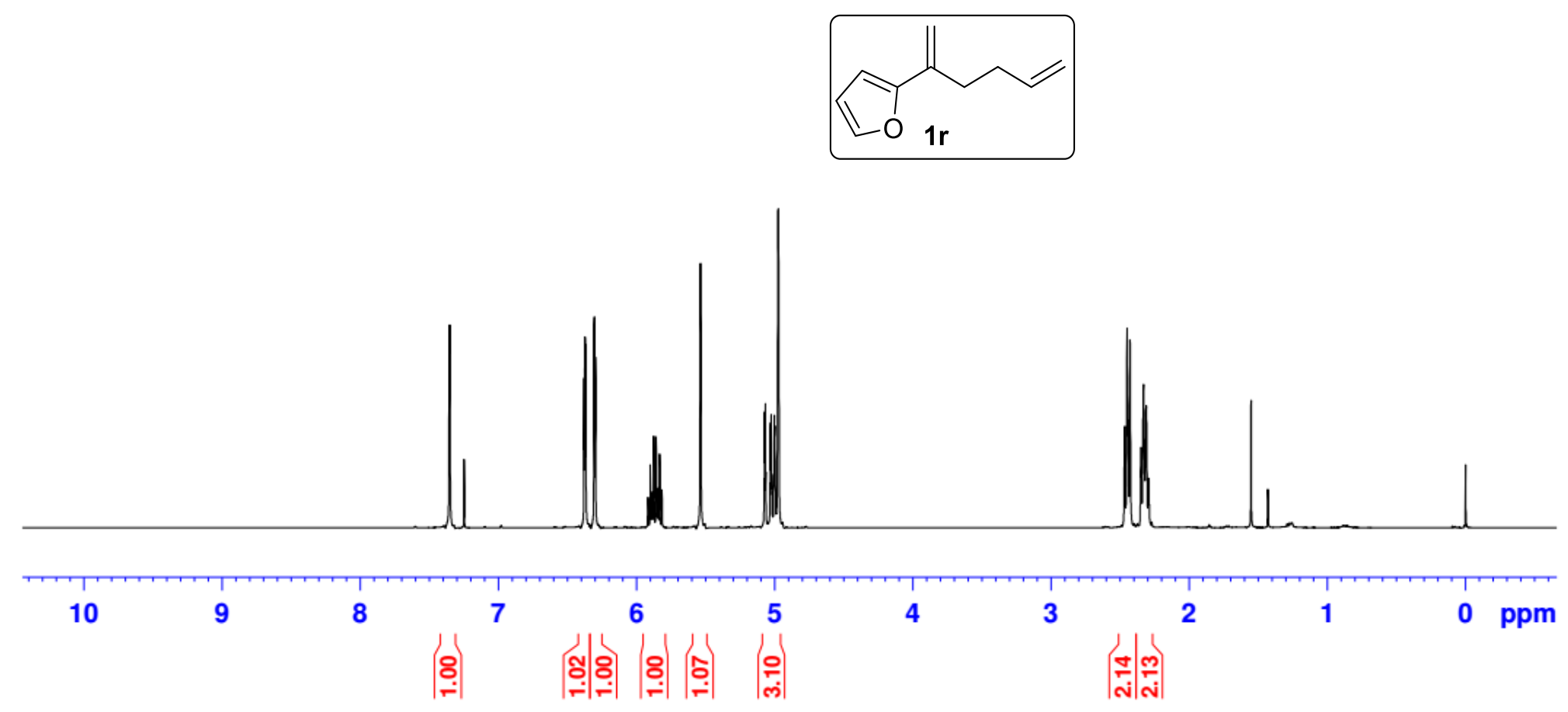




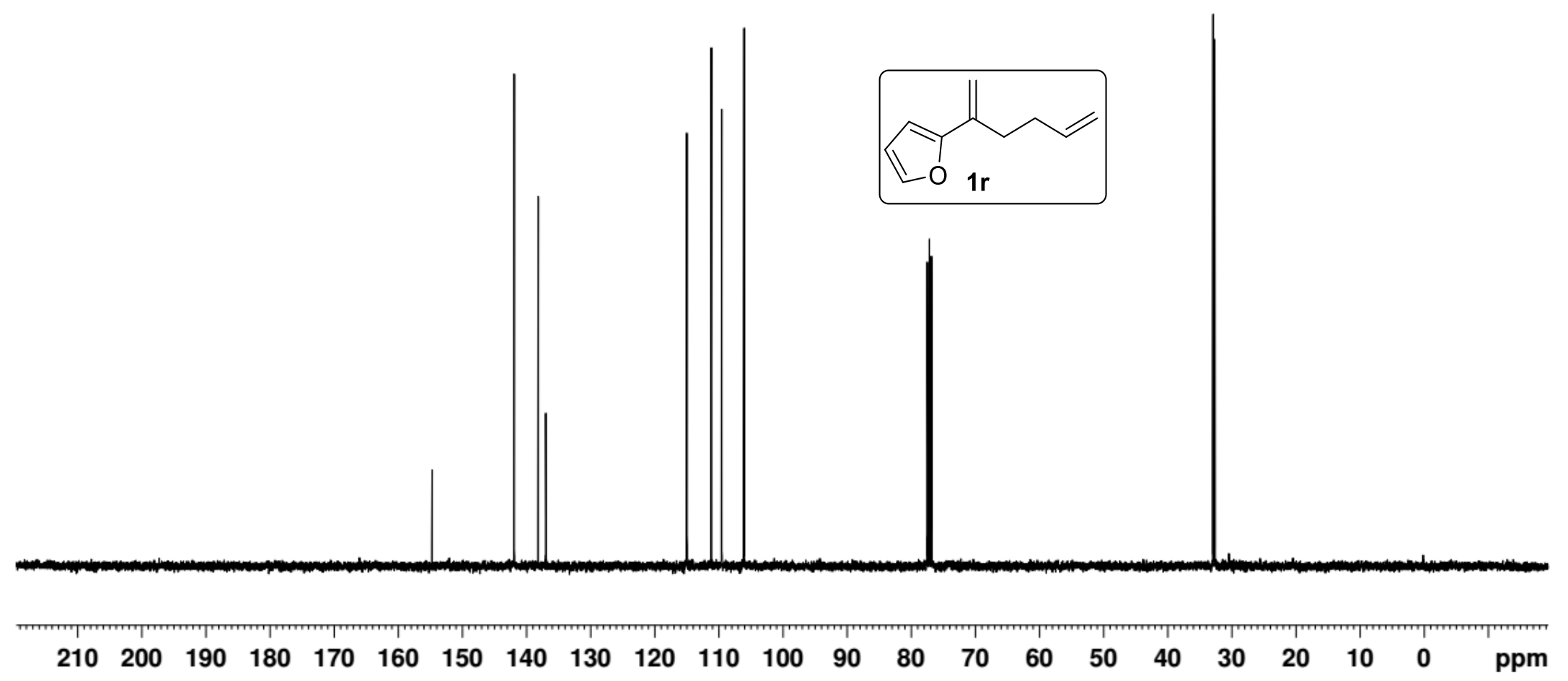


HY-X180401-3-CDC13-HNMR

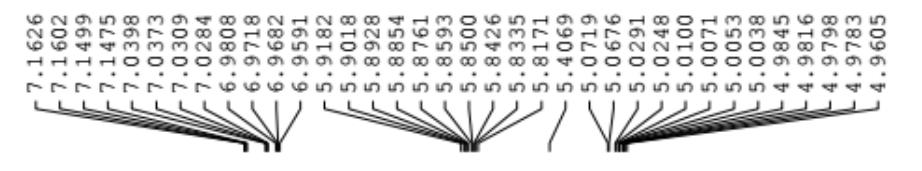

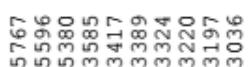

inininina

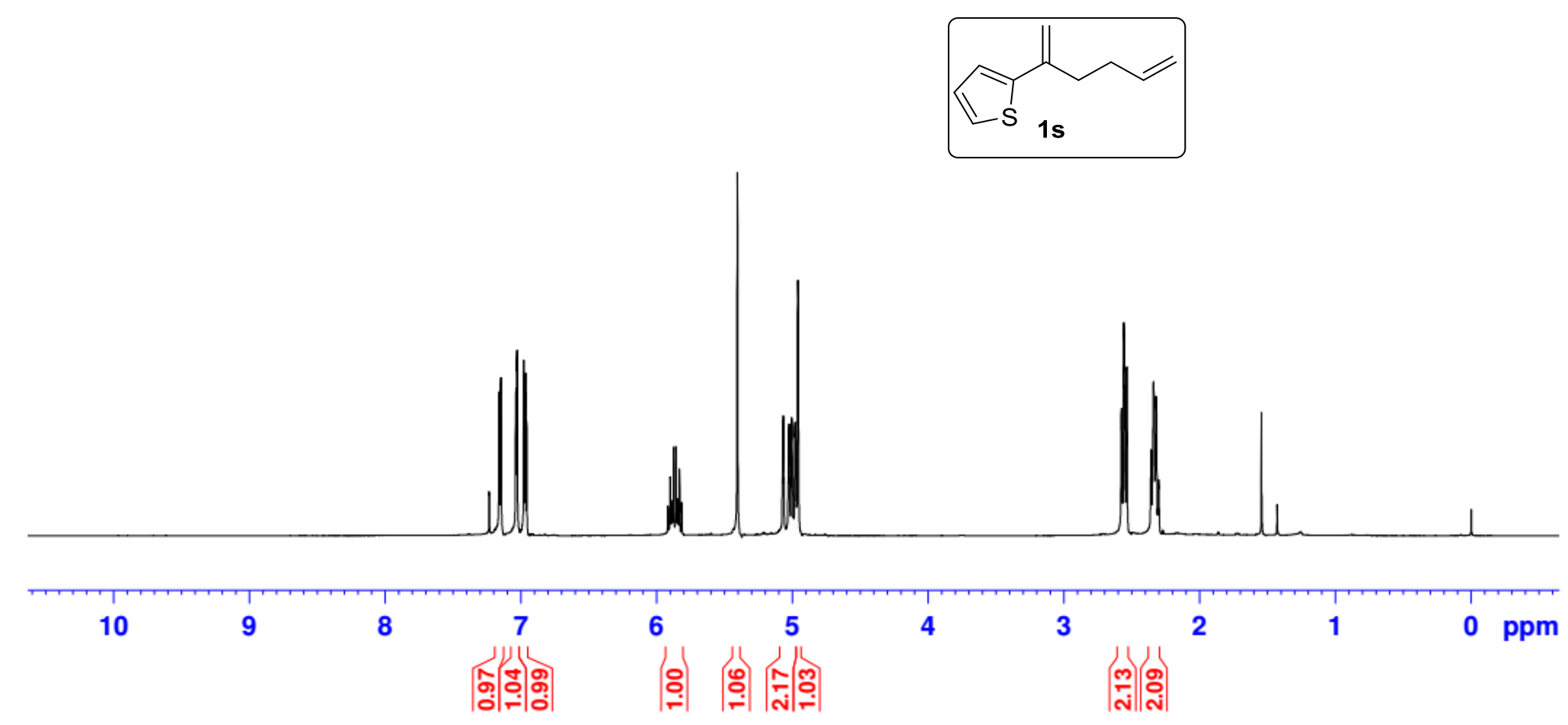


HY-X180401-3-CDC13-CNMR
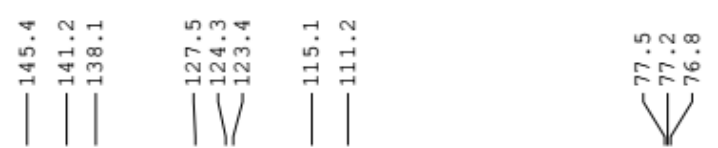

ㅁ.

$\mid$

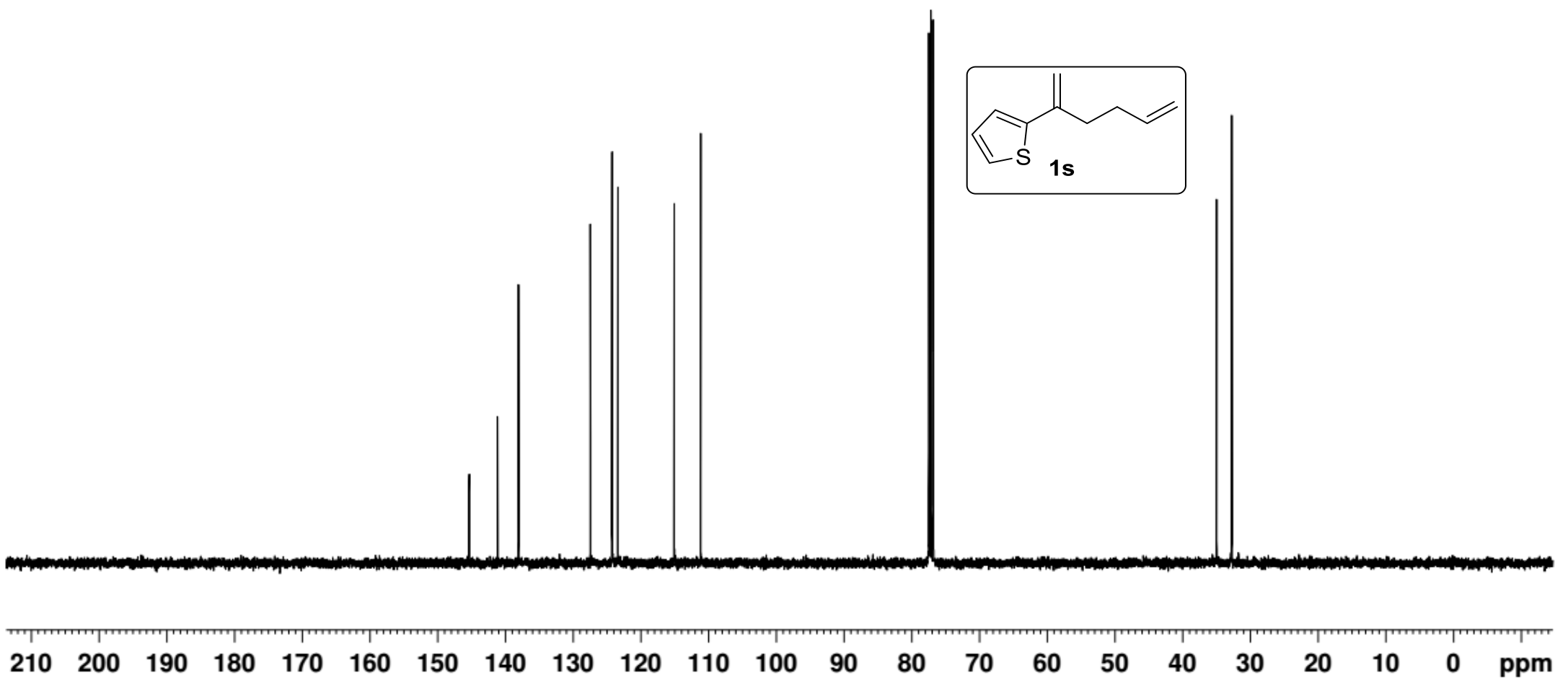



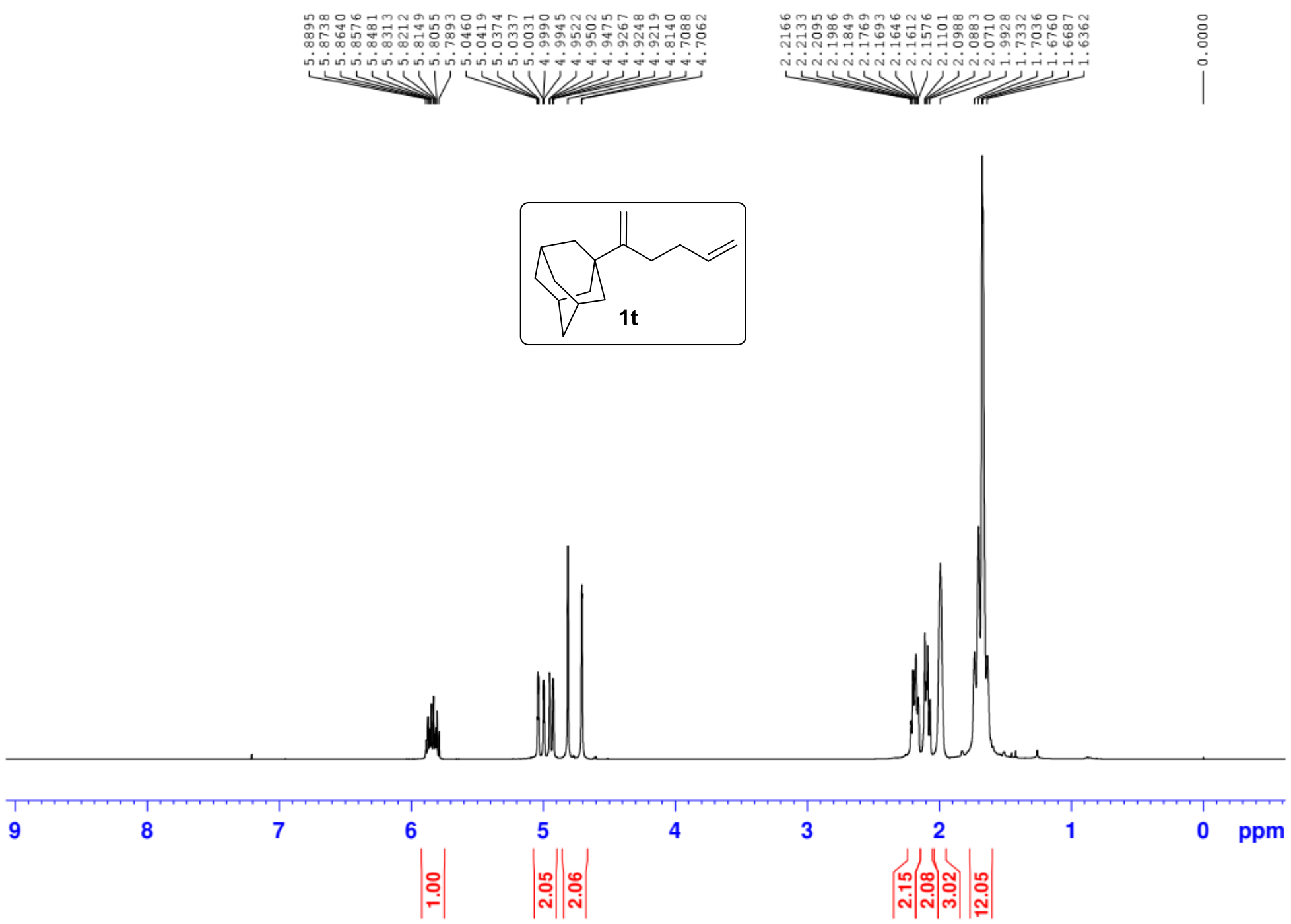
HY-X180318-1-CDC13-CNMR
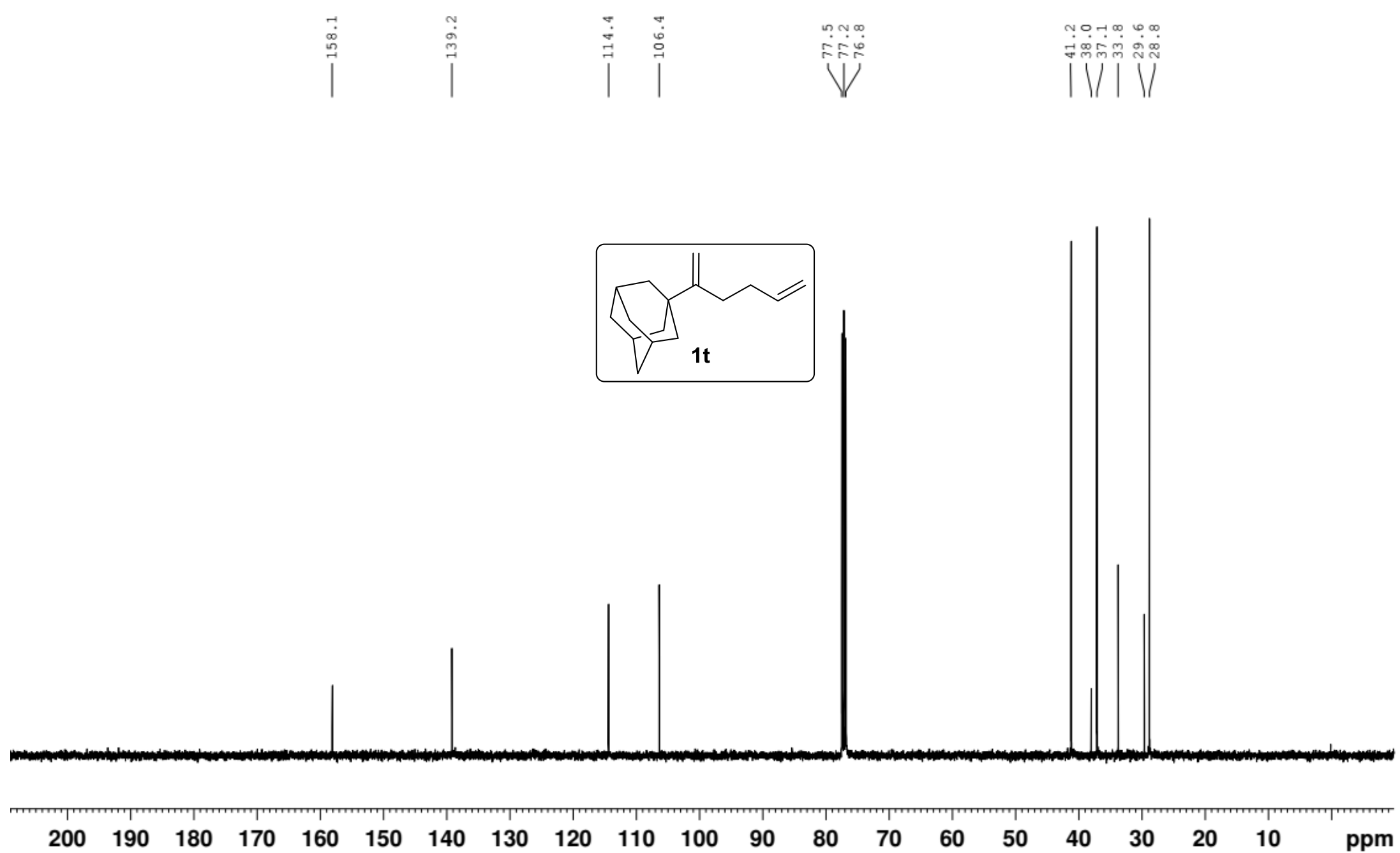
GB-X180129-1-0-HNMR
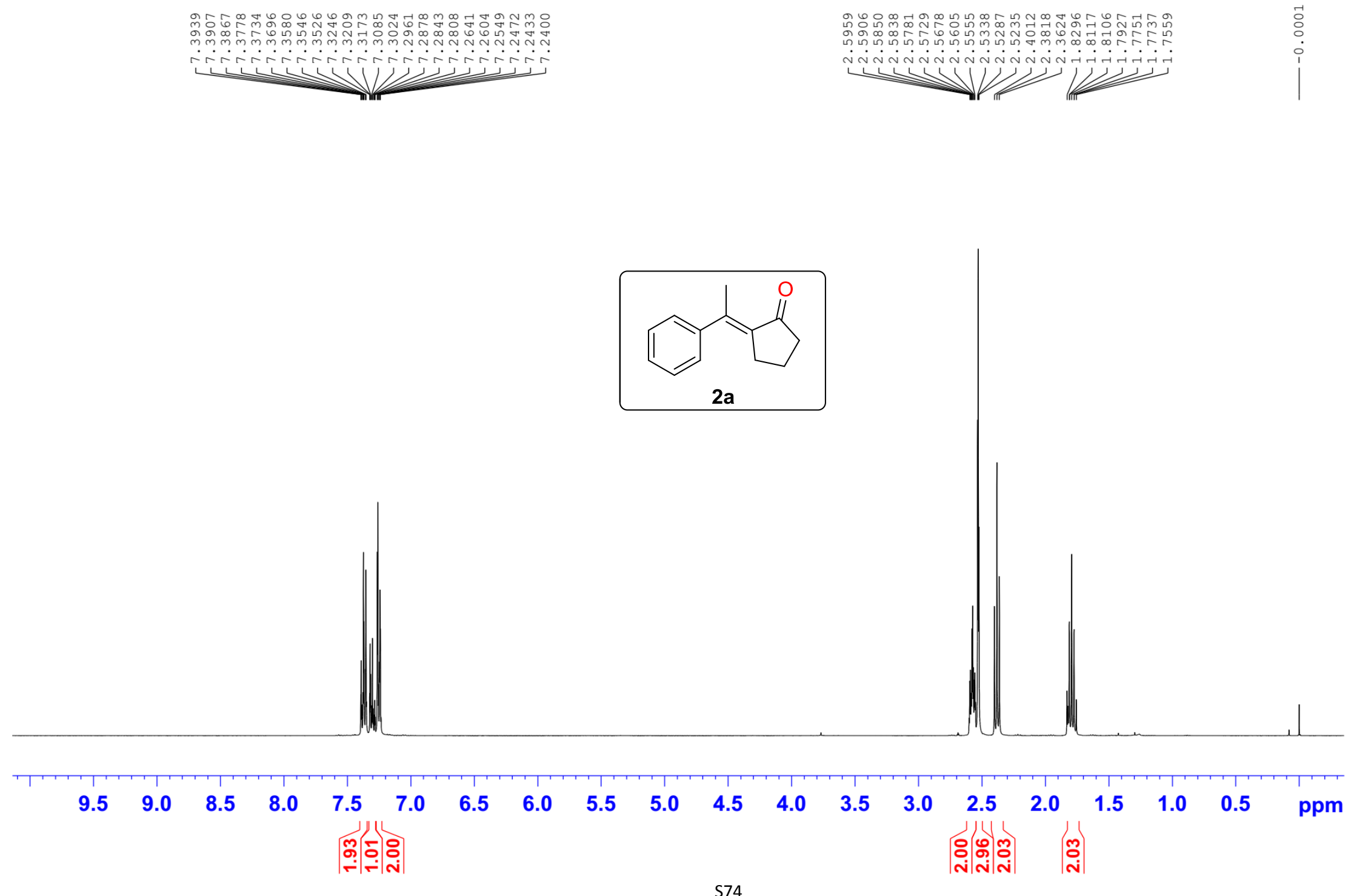
GB-X180129-1-0-CNMR
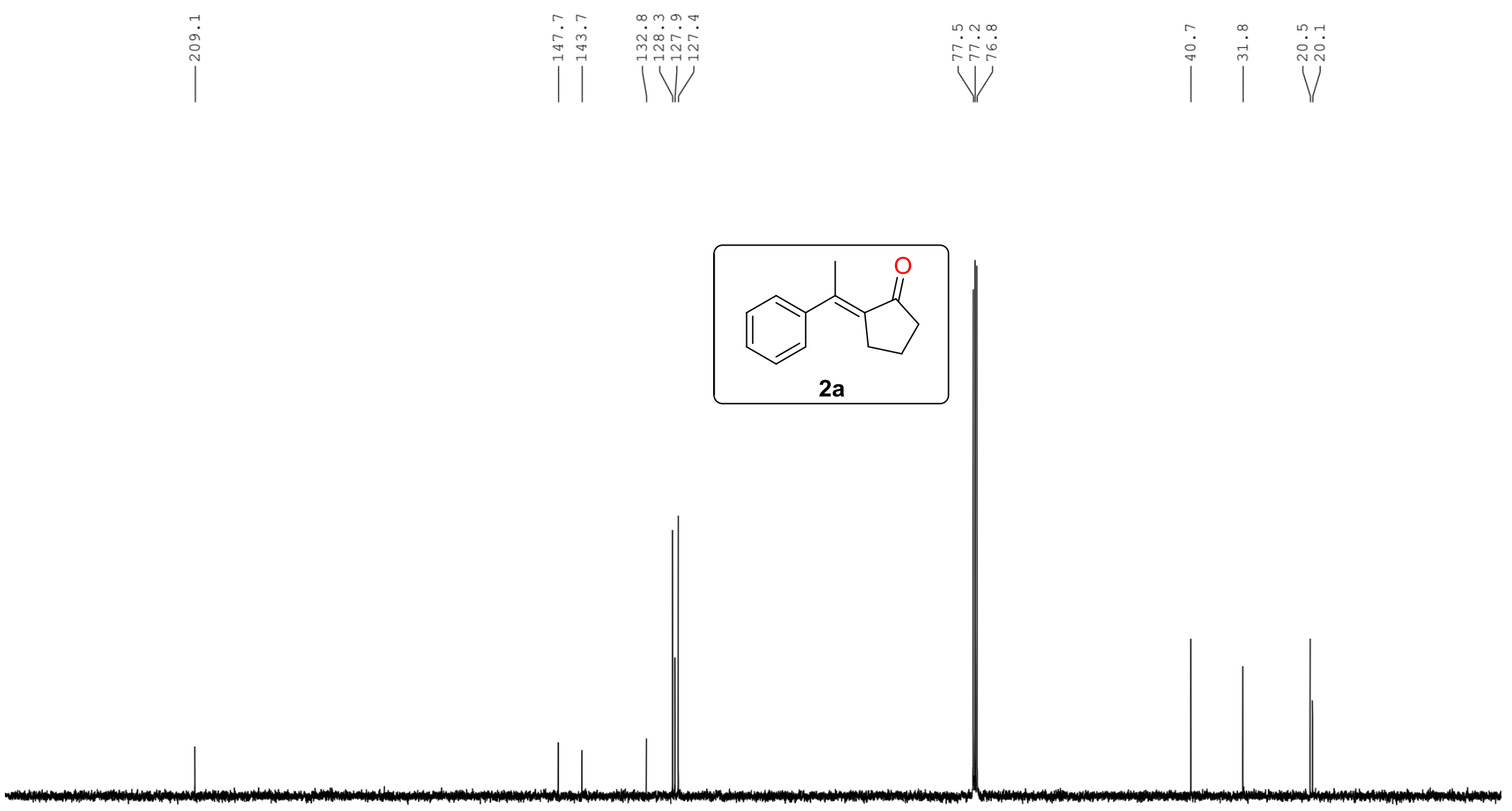
ZSC-X18X25-2a-cis-CDC13-HNMR

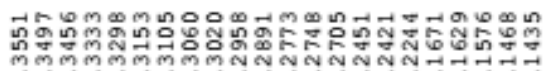

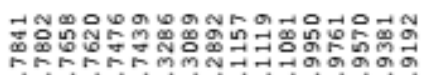

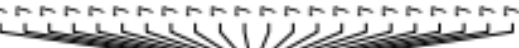

NNNNNNNNHJIJ
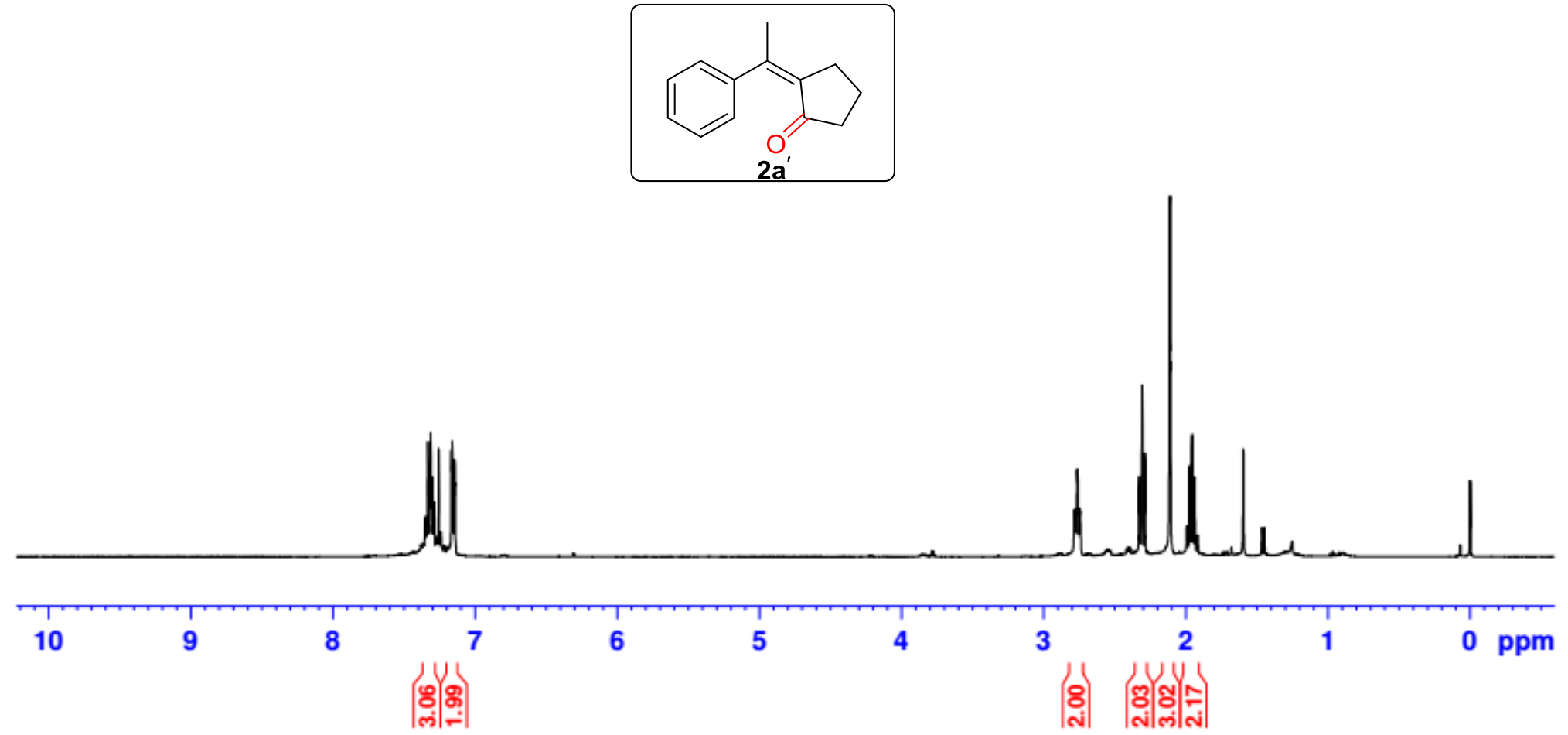


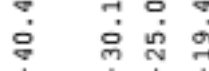

| $\mid$ | |

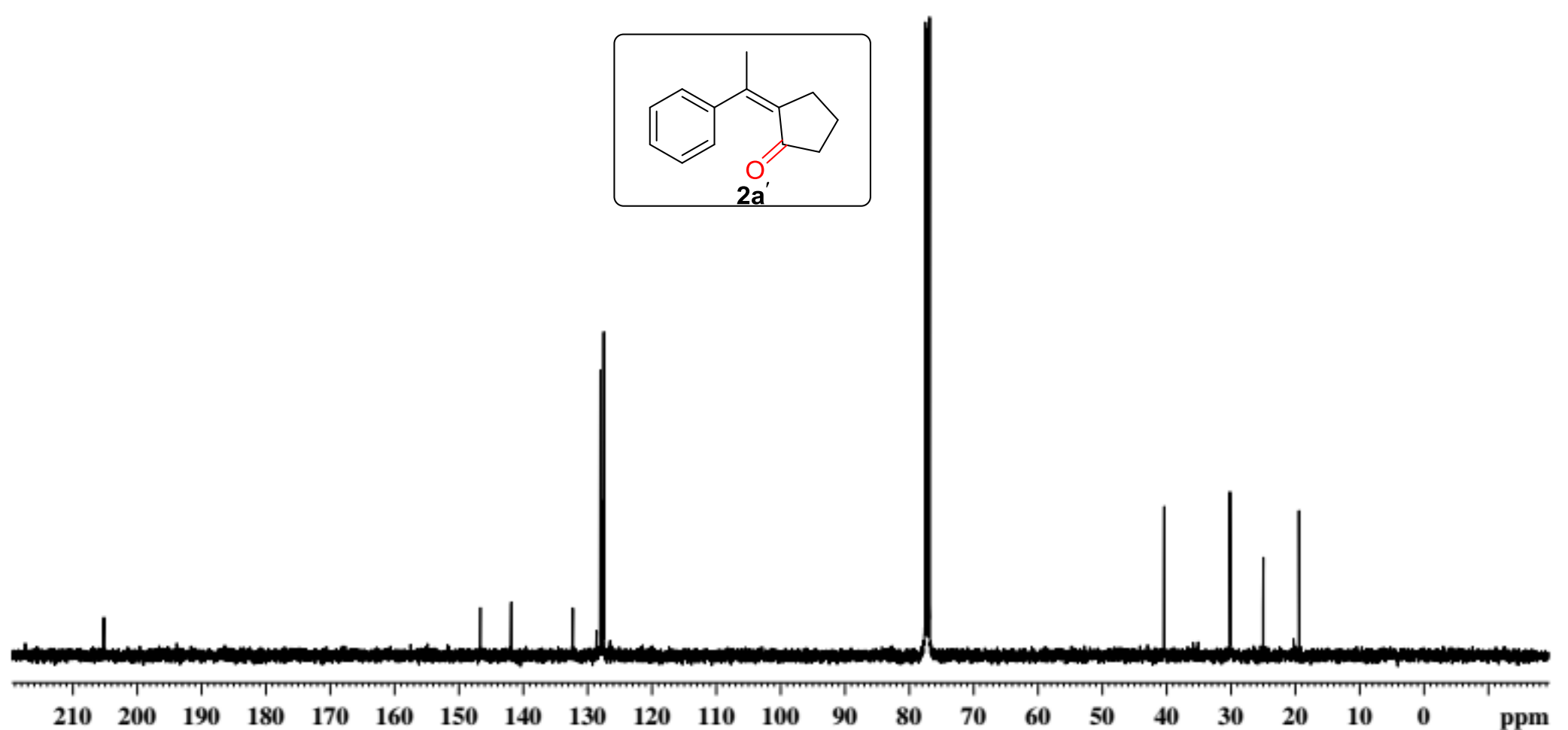


GB-X180129-1-1-HNMR

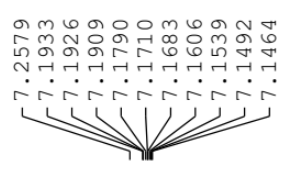

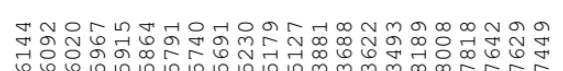

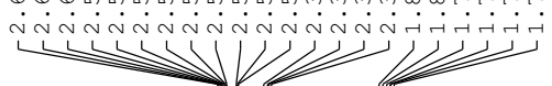
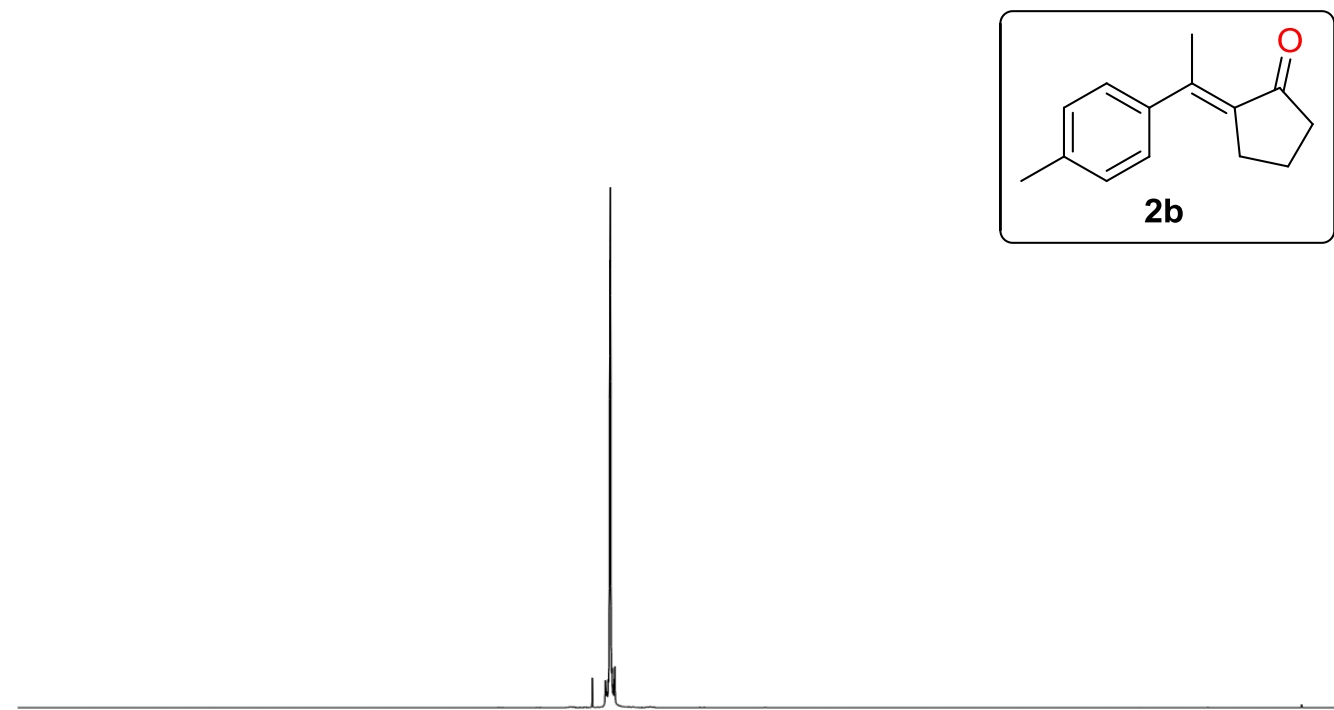

$2 b$
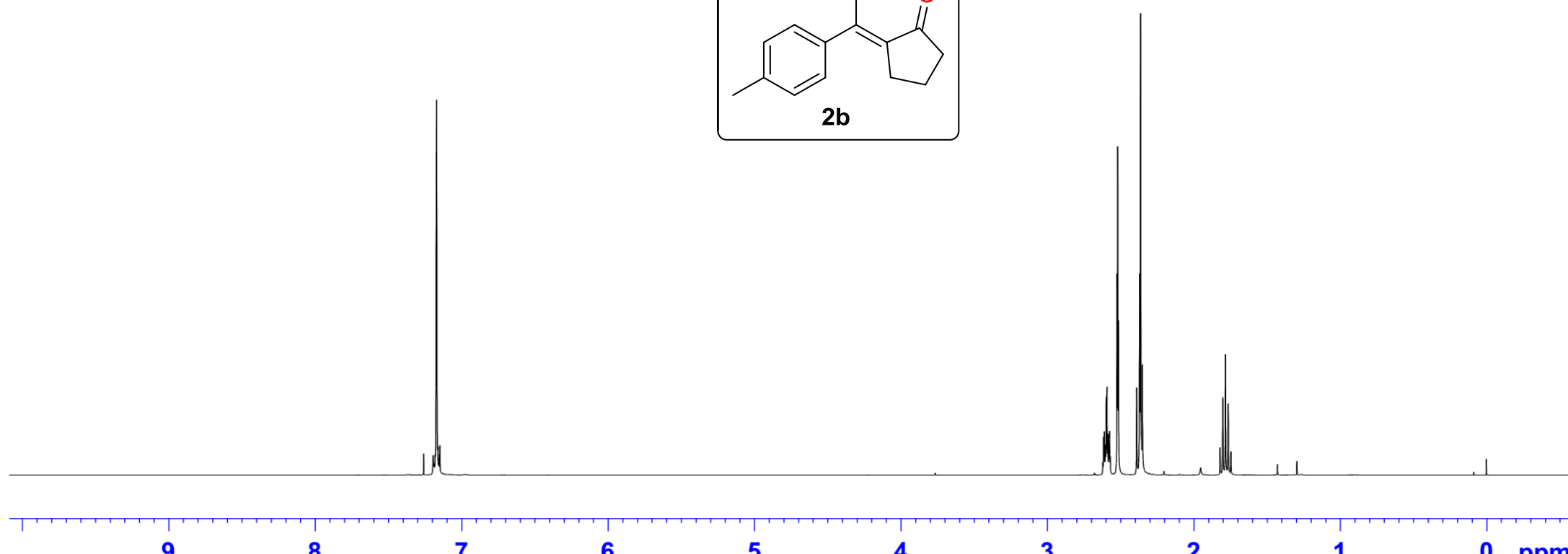

8

7

6

5

$\left(\begin{array}{c}0 \\ \hdashline \\ \dot{q}\end{array}\right)$

3

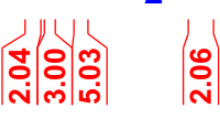


GB-X180129-1-1-CNMR
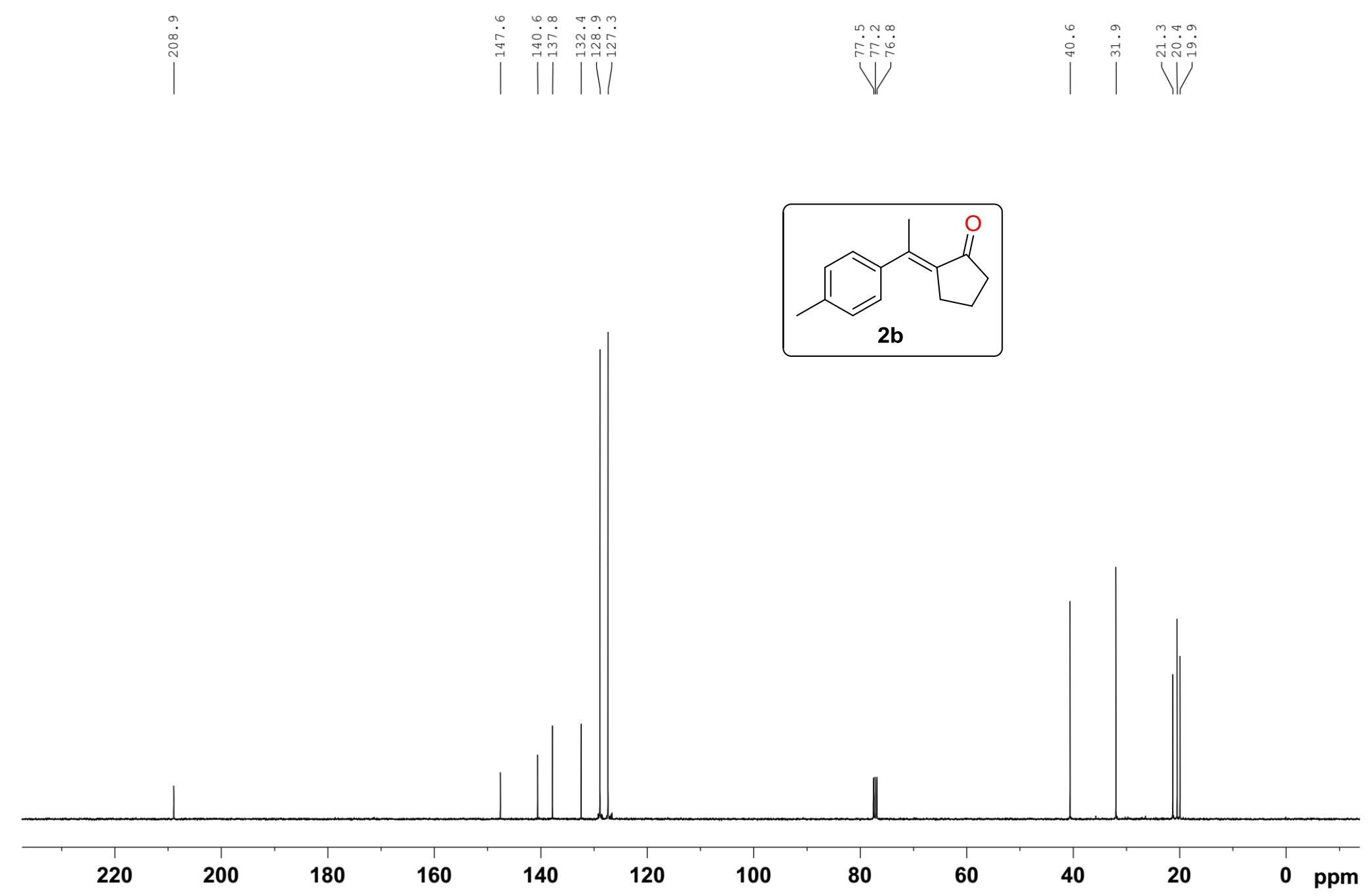
GB-X180129-1-2-3Me-HNMR
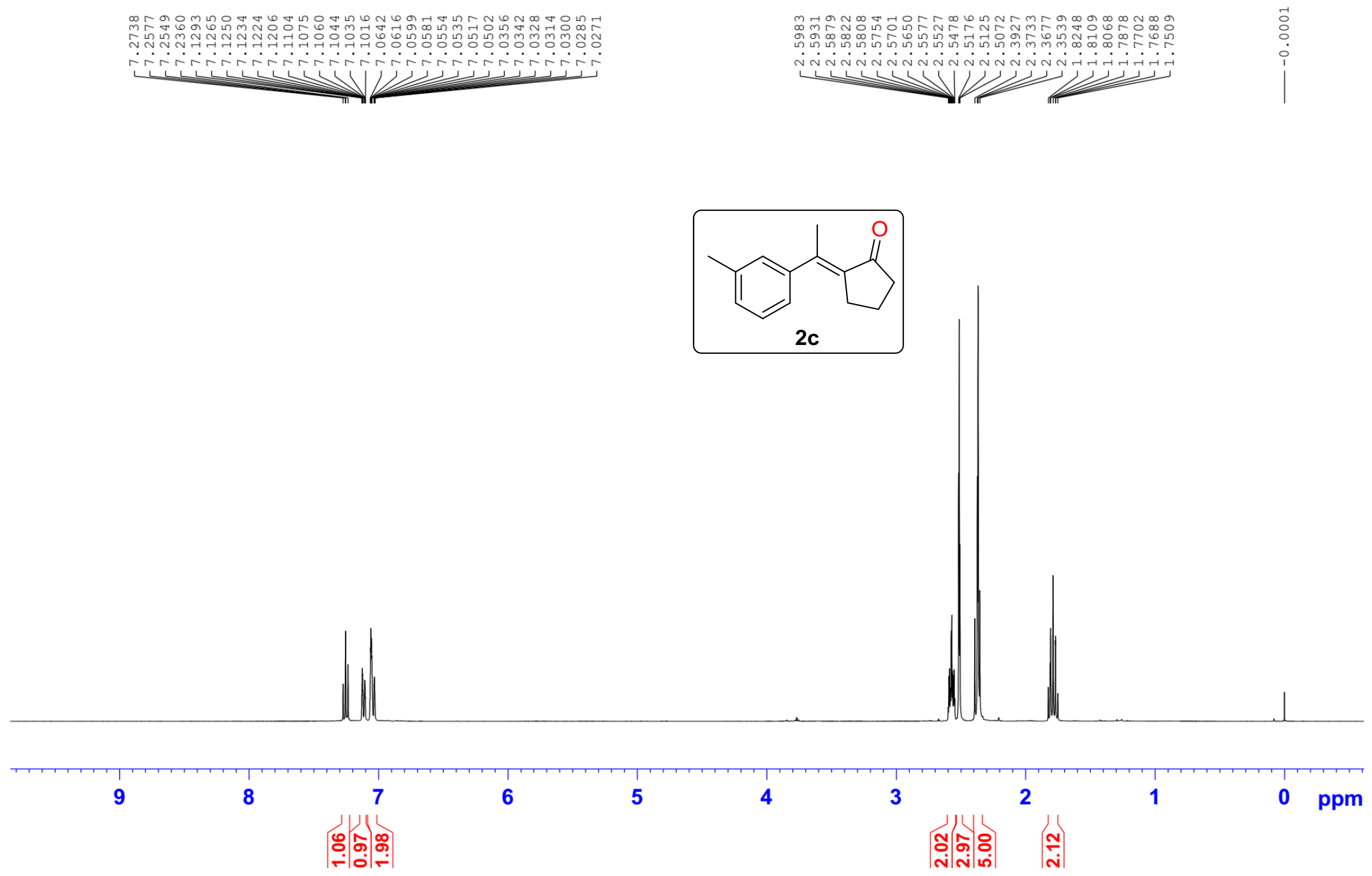

S80

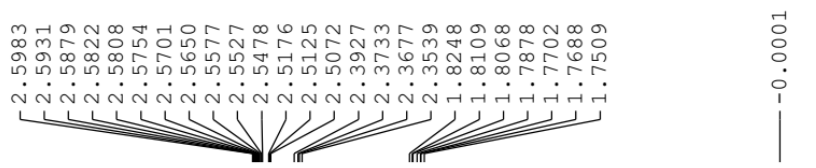

ก|ก| 
GB-X180129-1-2-3Me-CNMR

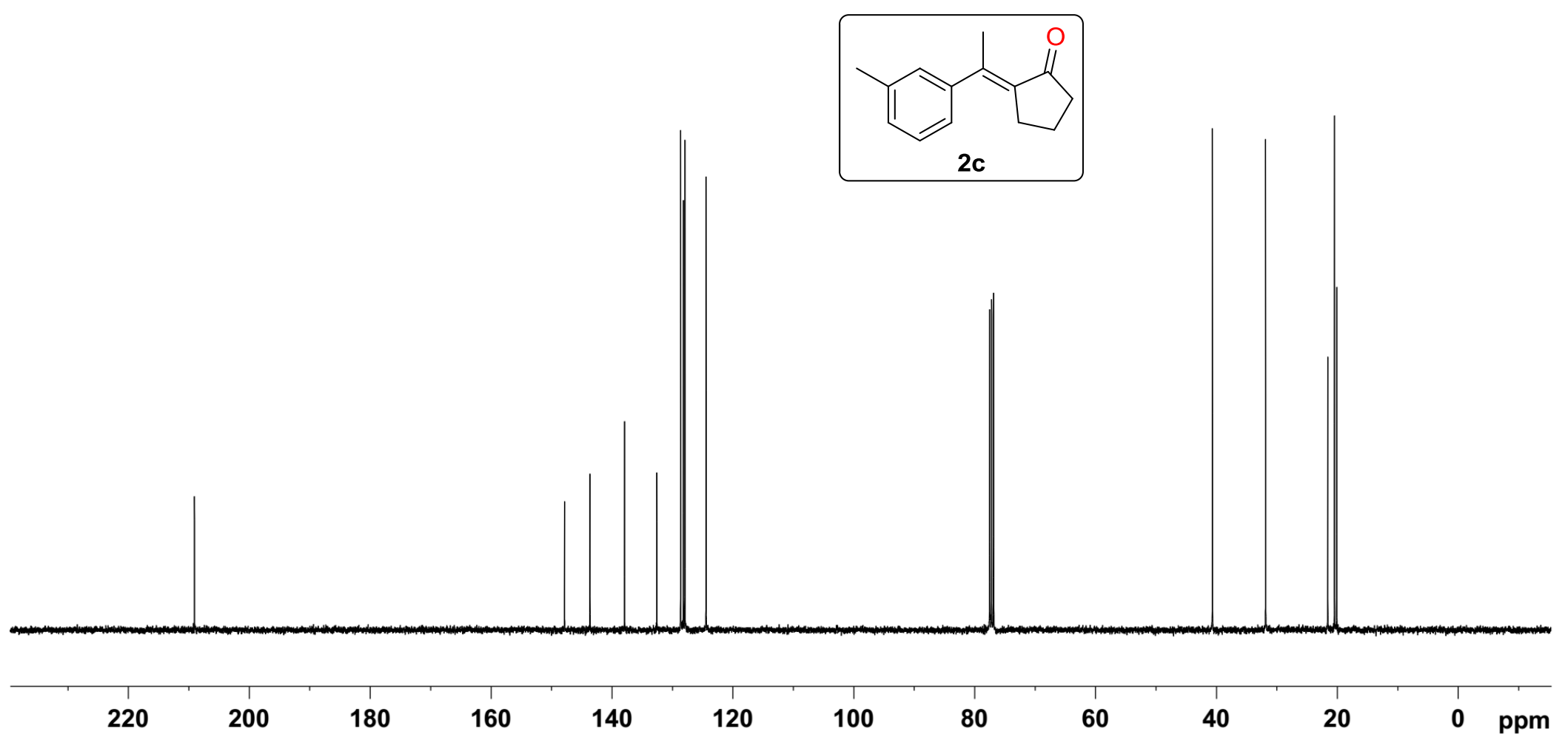


GB-X180308-1-4-2Me-HNMR

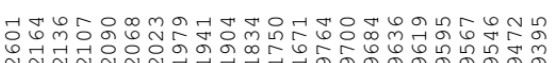

$\cdots$

(n)

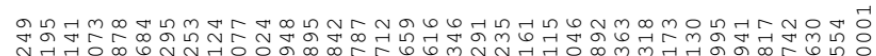

$\underbrace{4}$
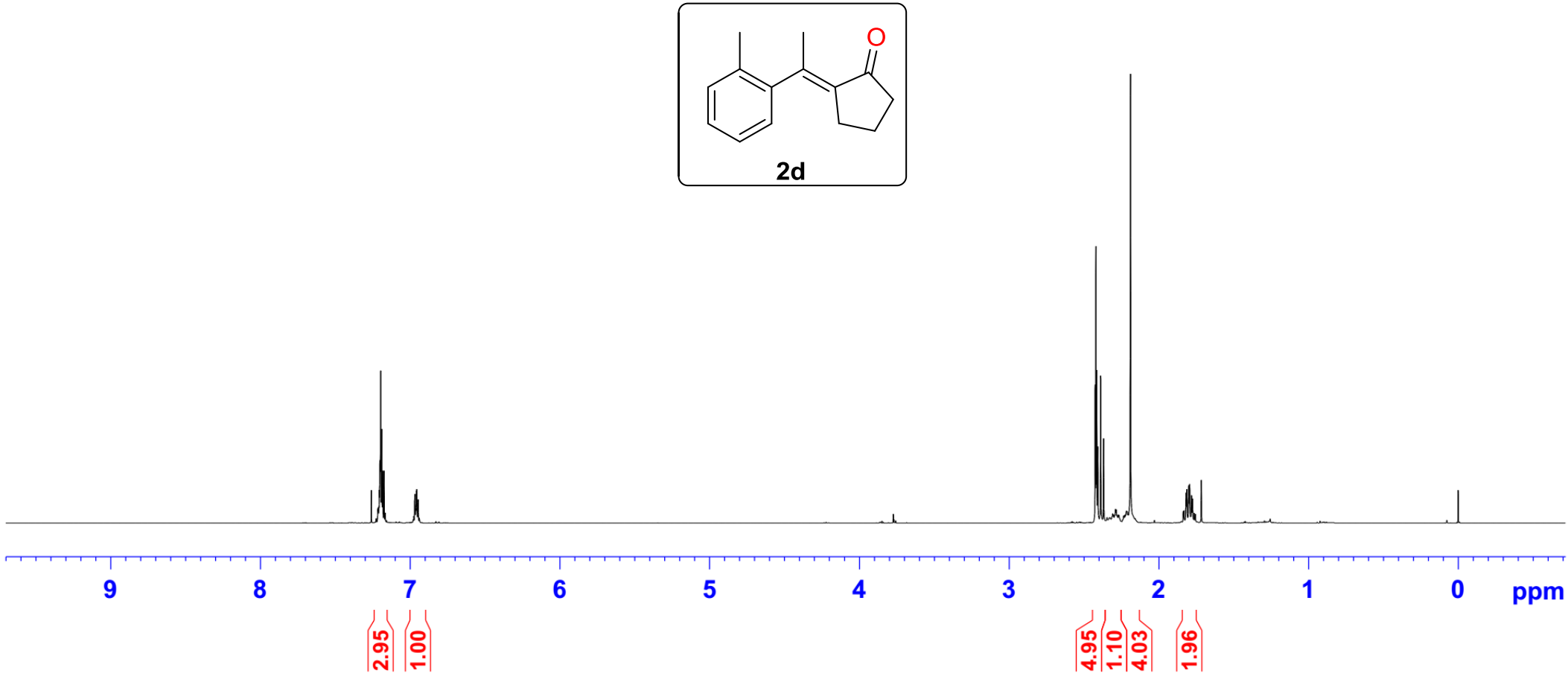
GB-X180308-1-4-2Me-CNMR
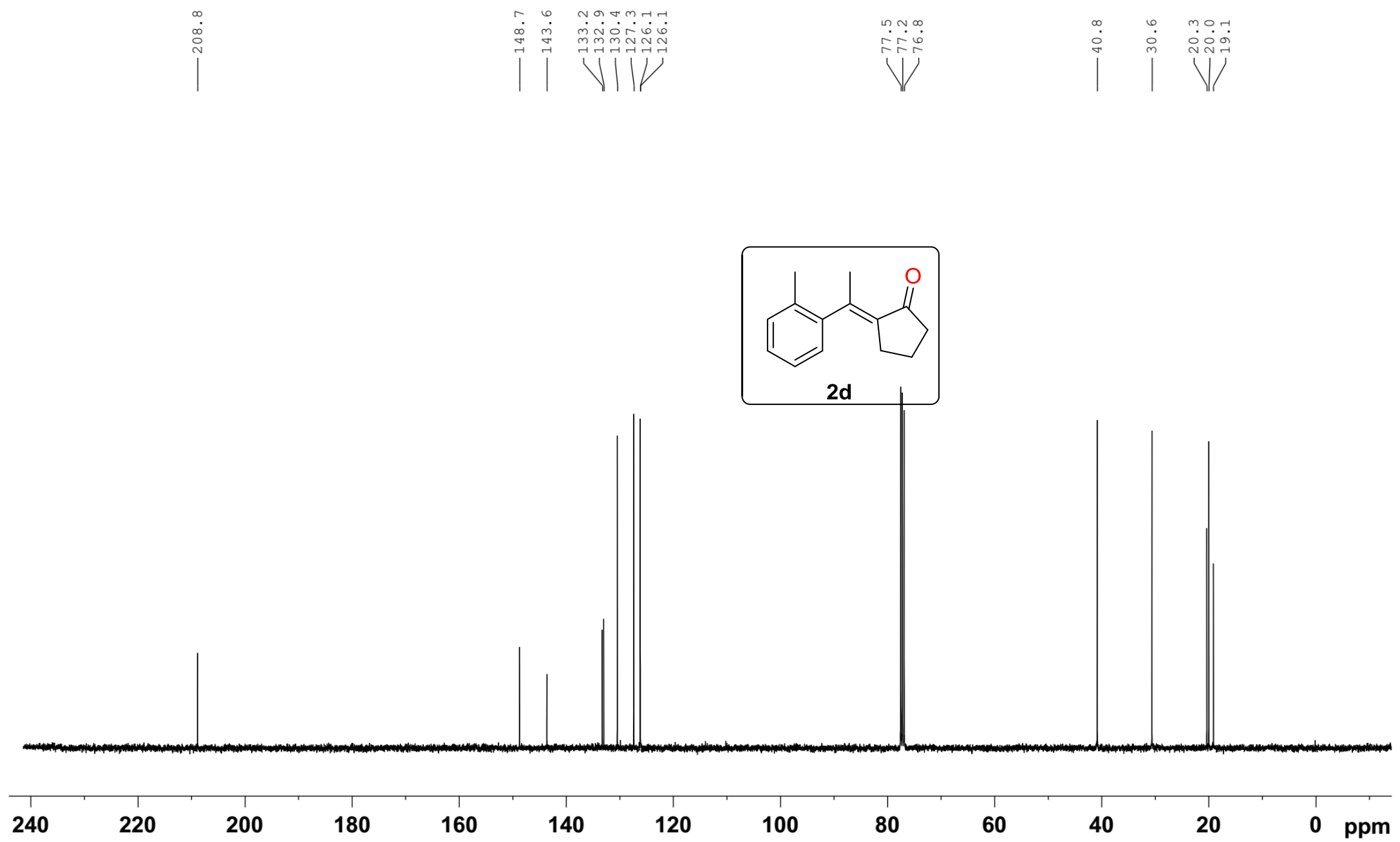
GB-X180129-1-4-HNMR
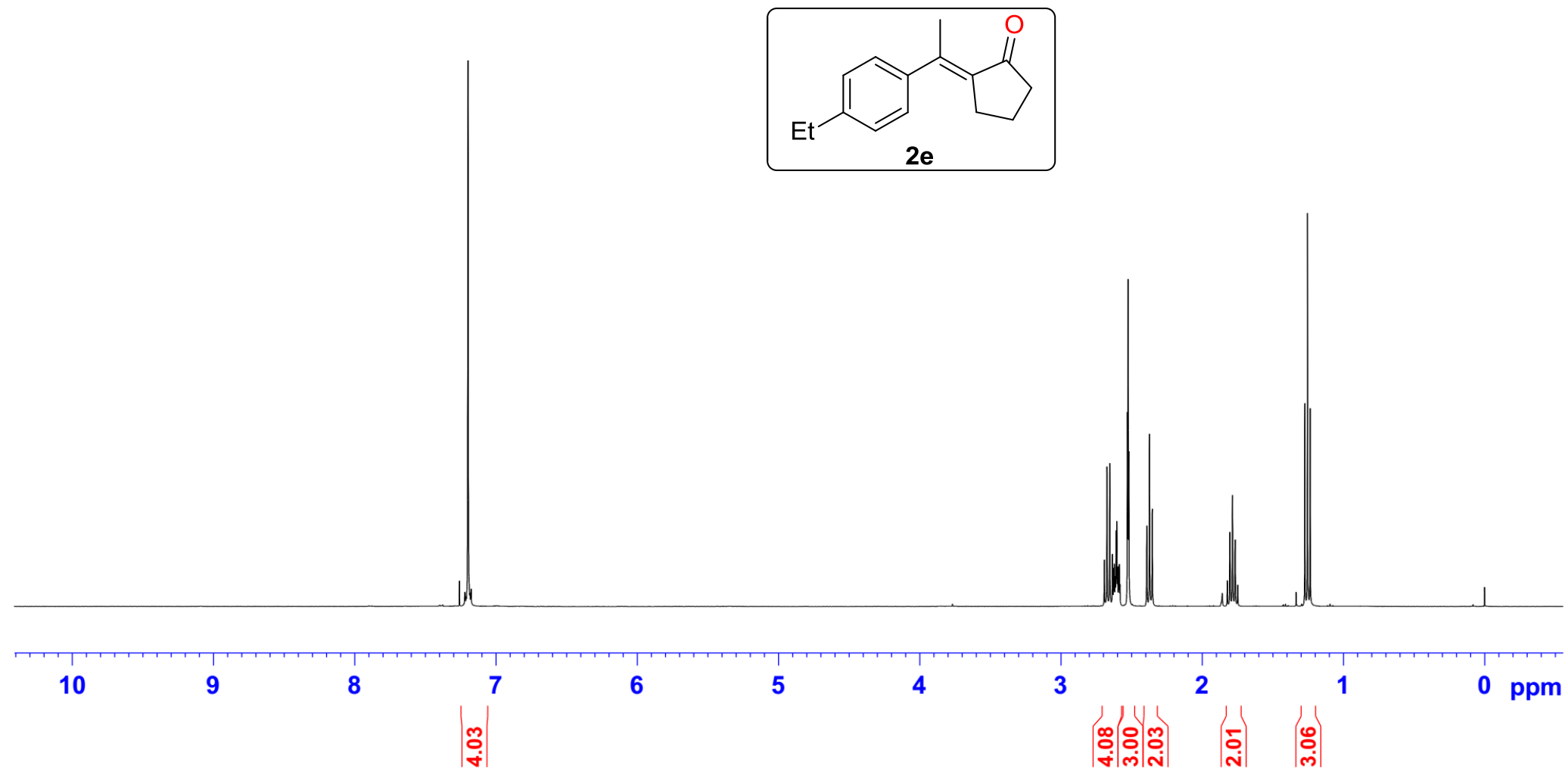
GB-X180129-1-4-4Et-CNMR

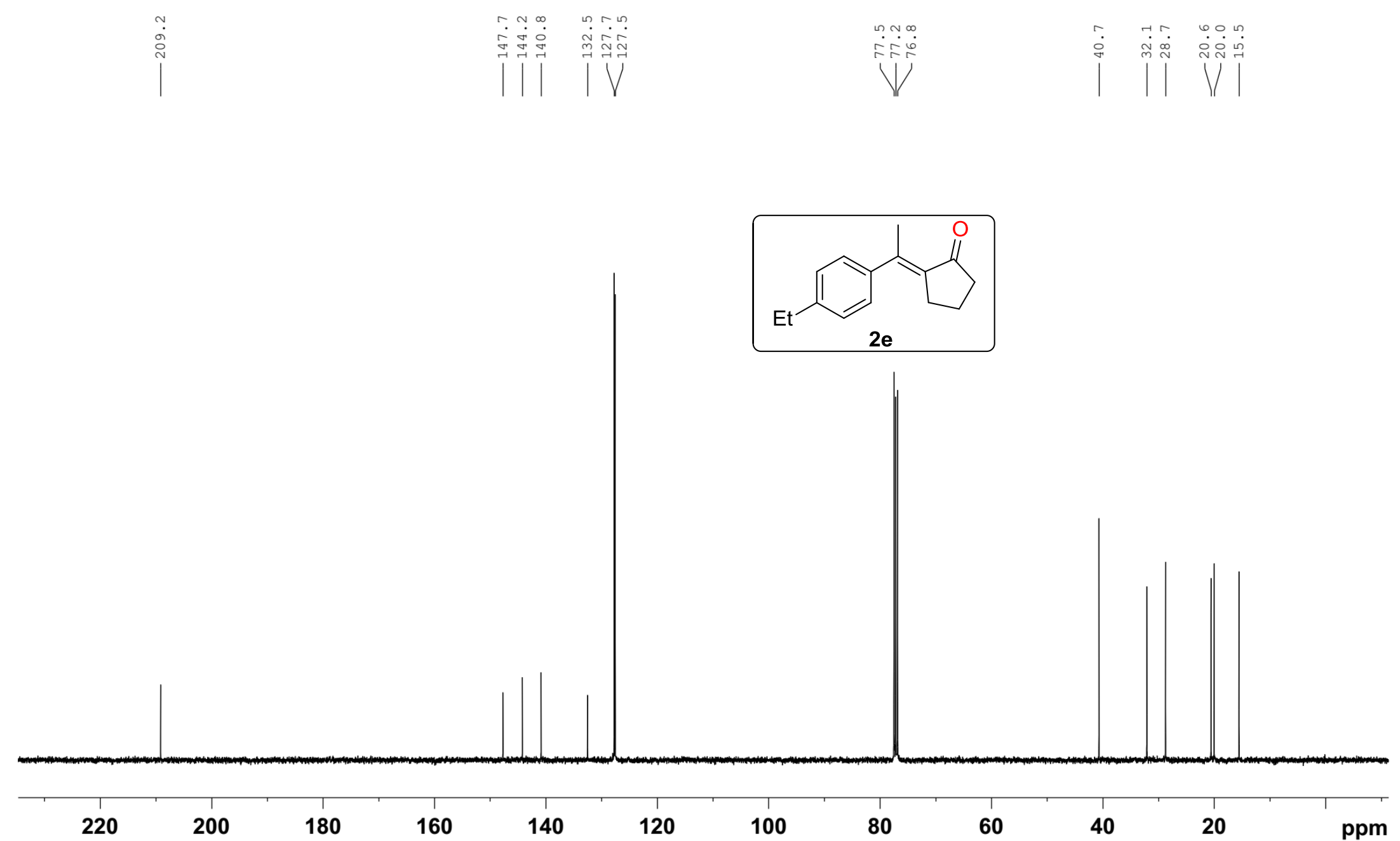


GB-X180129-1-5-HNMR
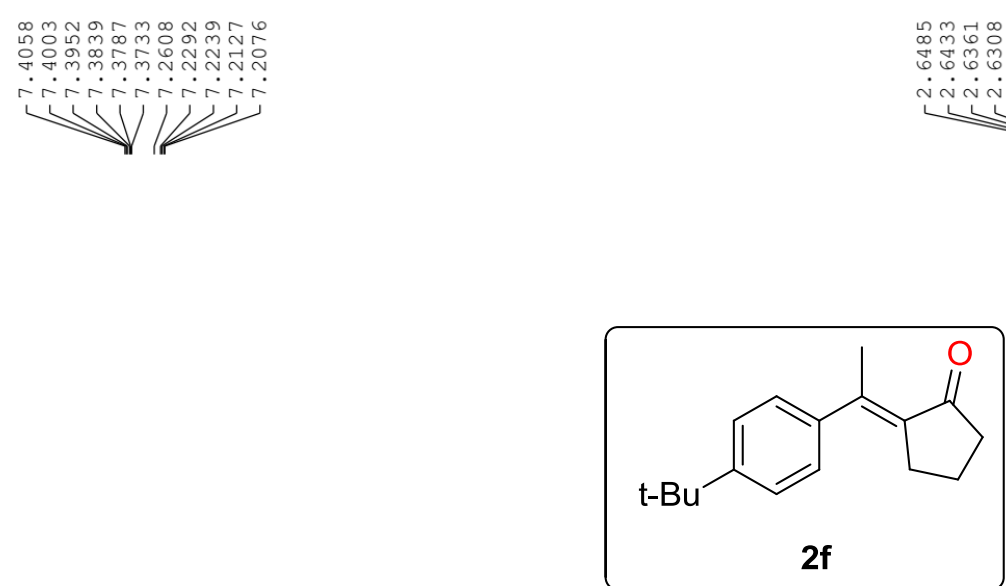

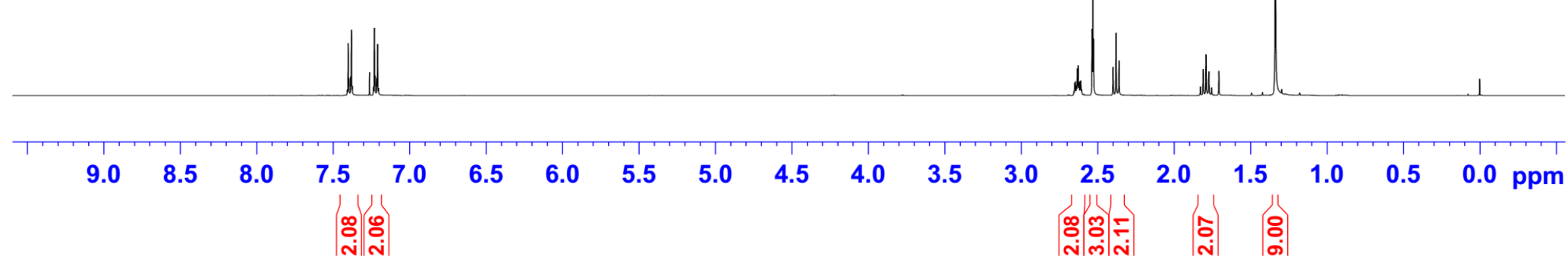


GB-X180129-1-5-CNMR
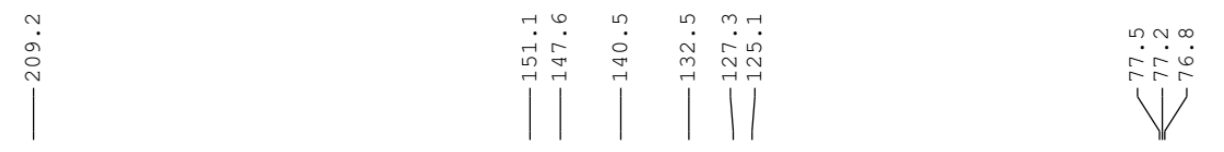

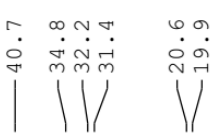
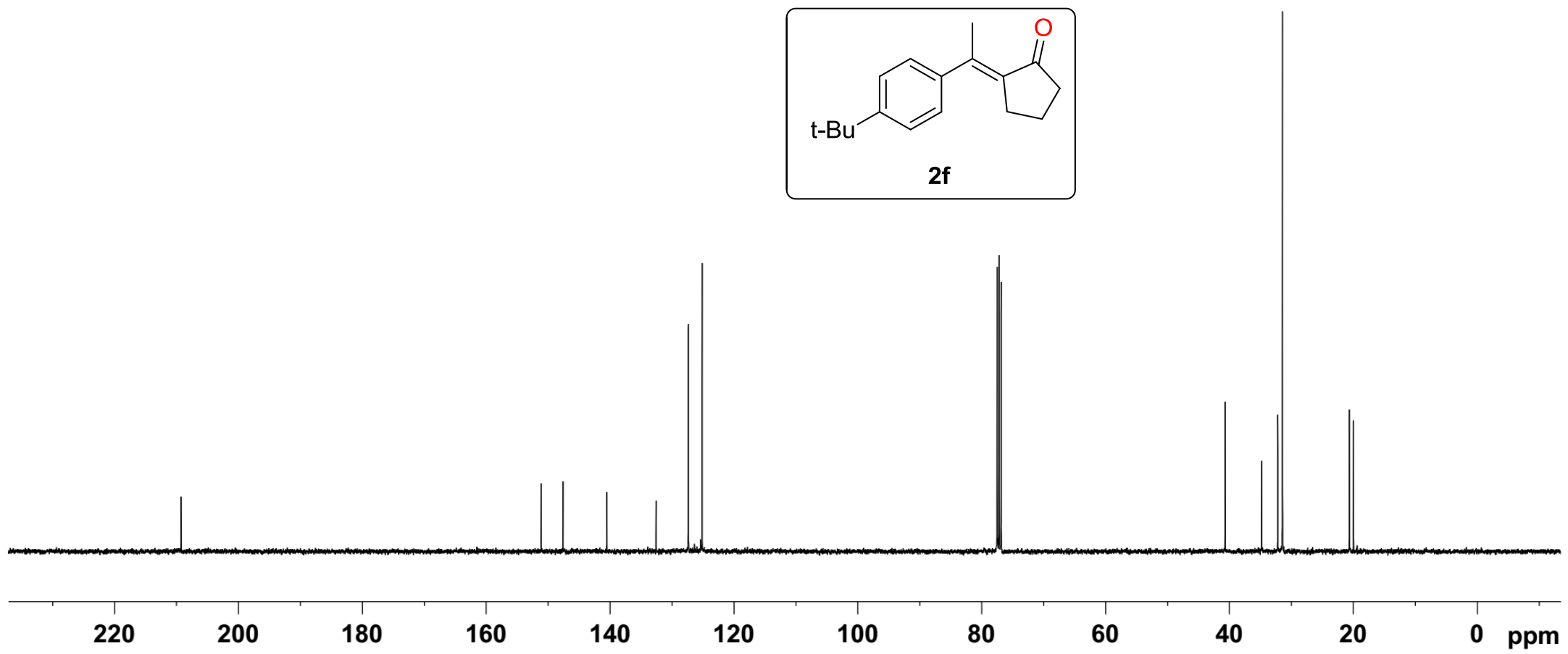
GB-X180614-4MeO-HNMR

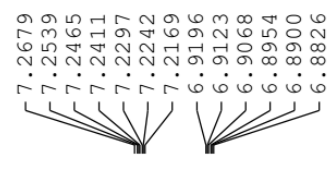

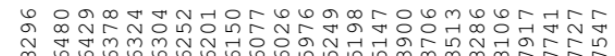

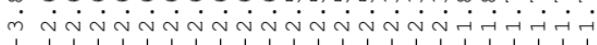

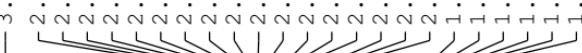

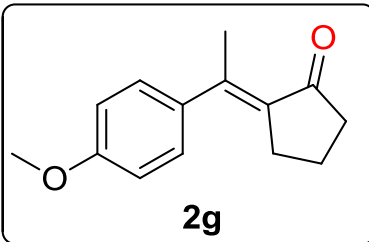

$2 \mathrm{~g}$

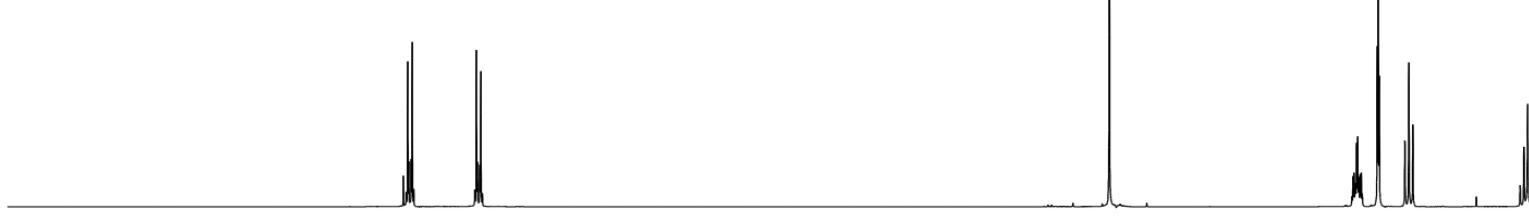

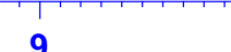

8

7

6

5

(ִ)

4

|)

3

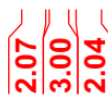

2

1

0 ppm 
GB-X180614-4MeO-CNMR
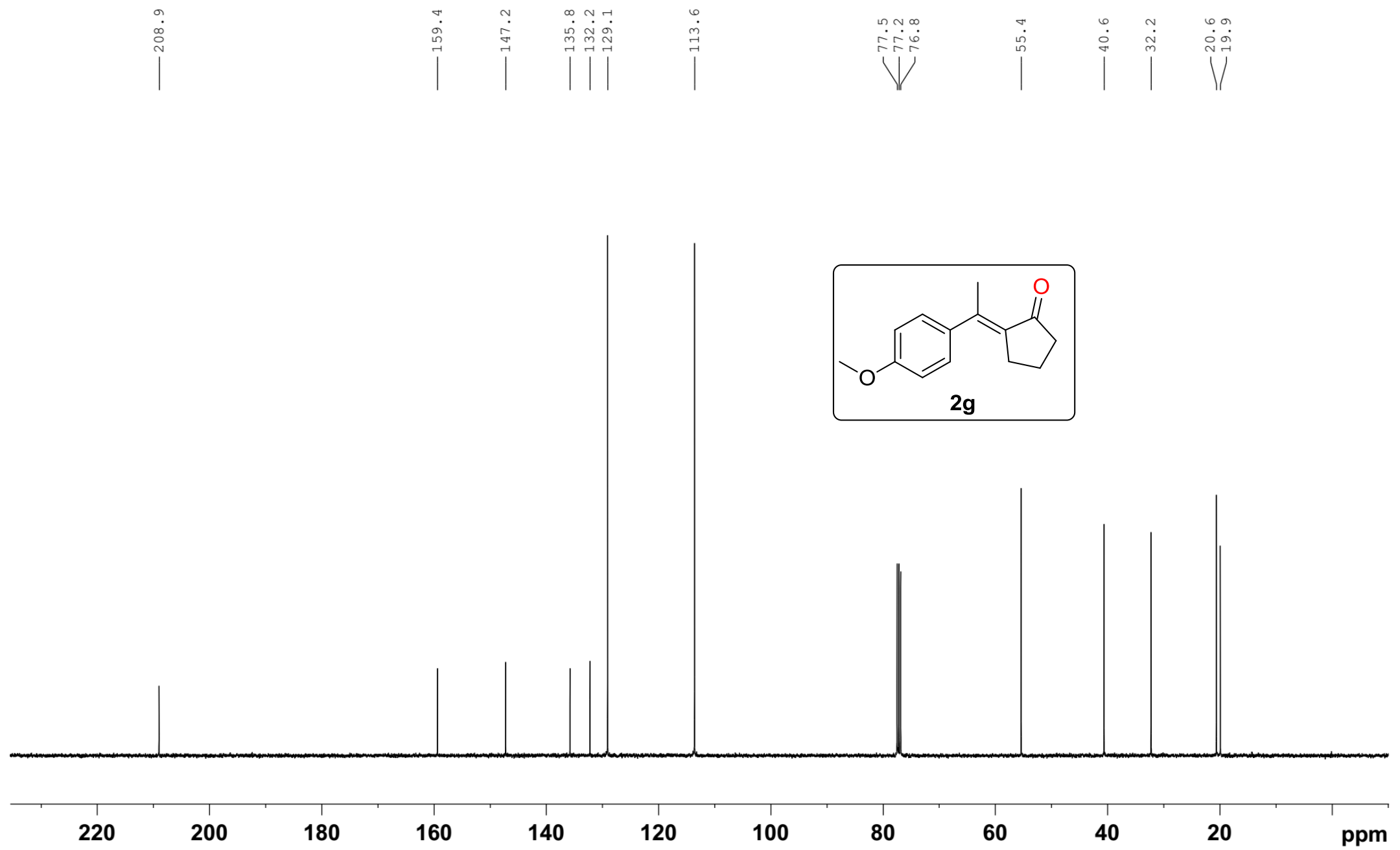
GB-X180402-3-1-4F-HNMR
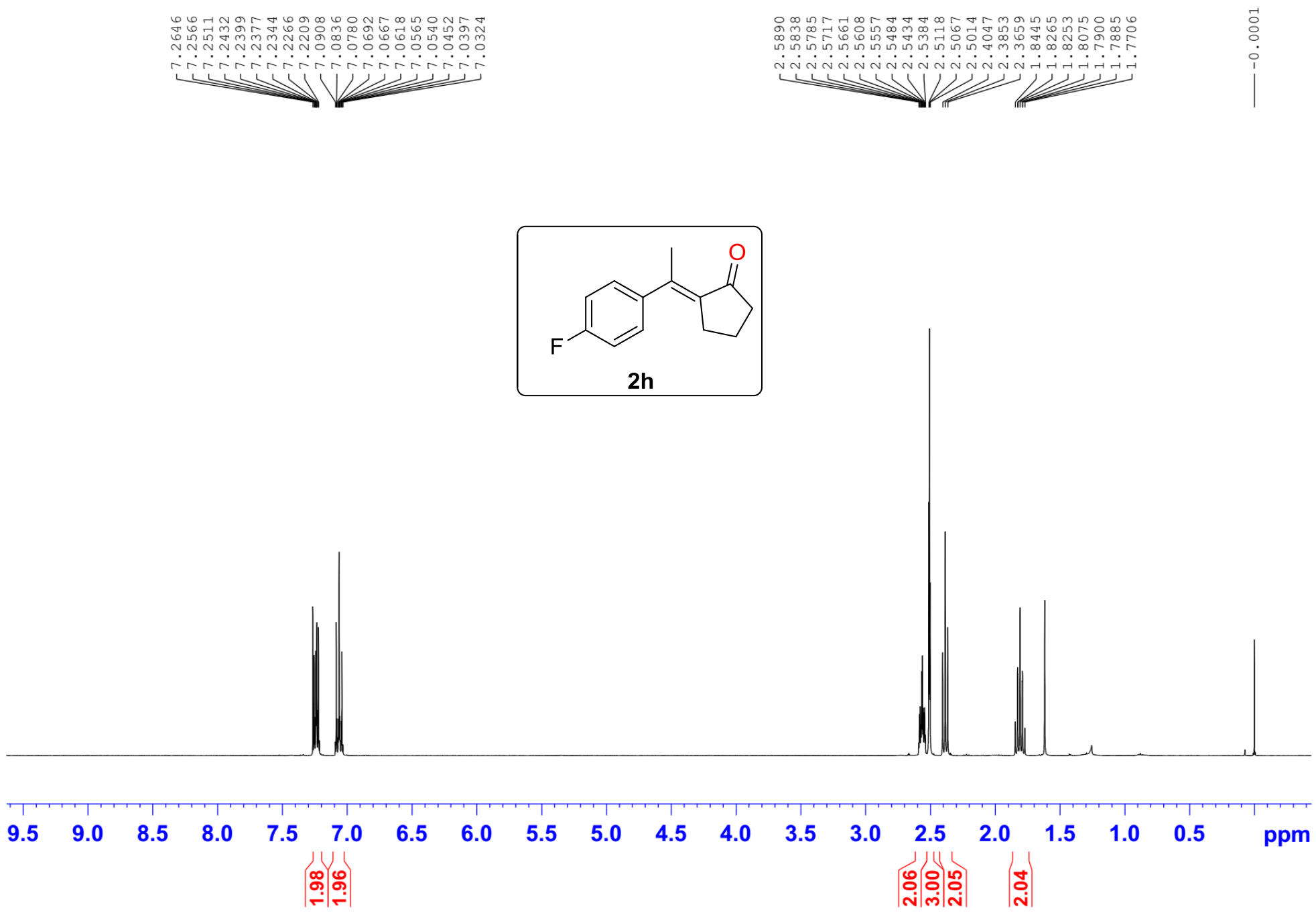
GB-X180402-3-1-4F-CNMR
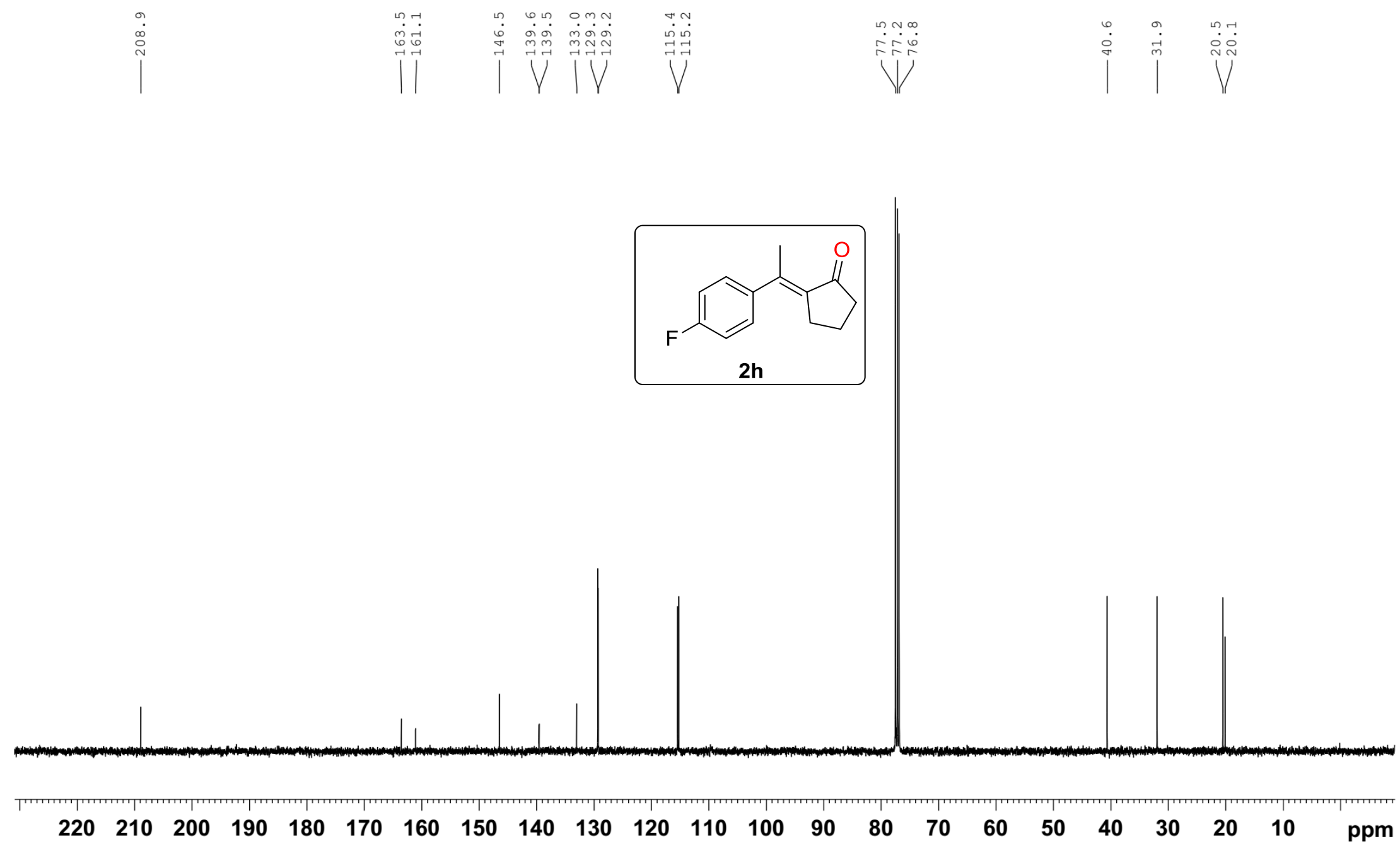
GB-X180402-3-1-4F-FNMR
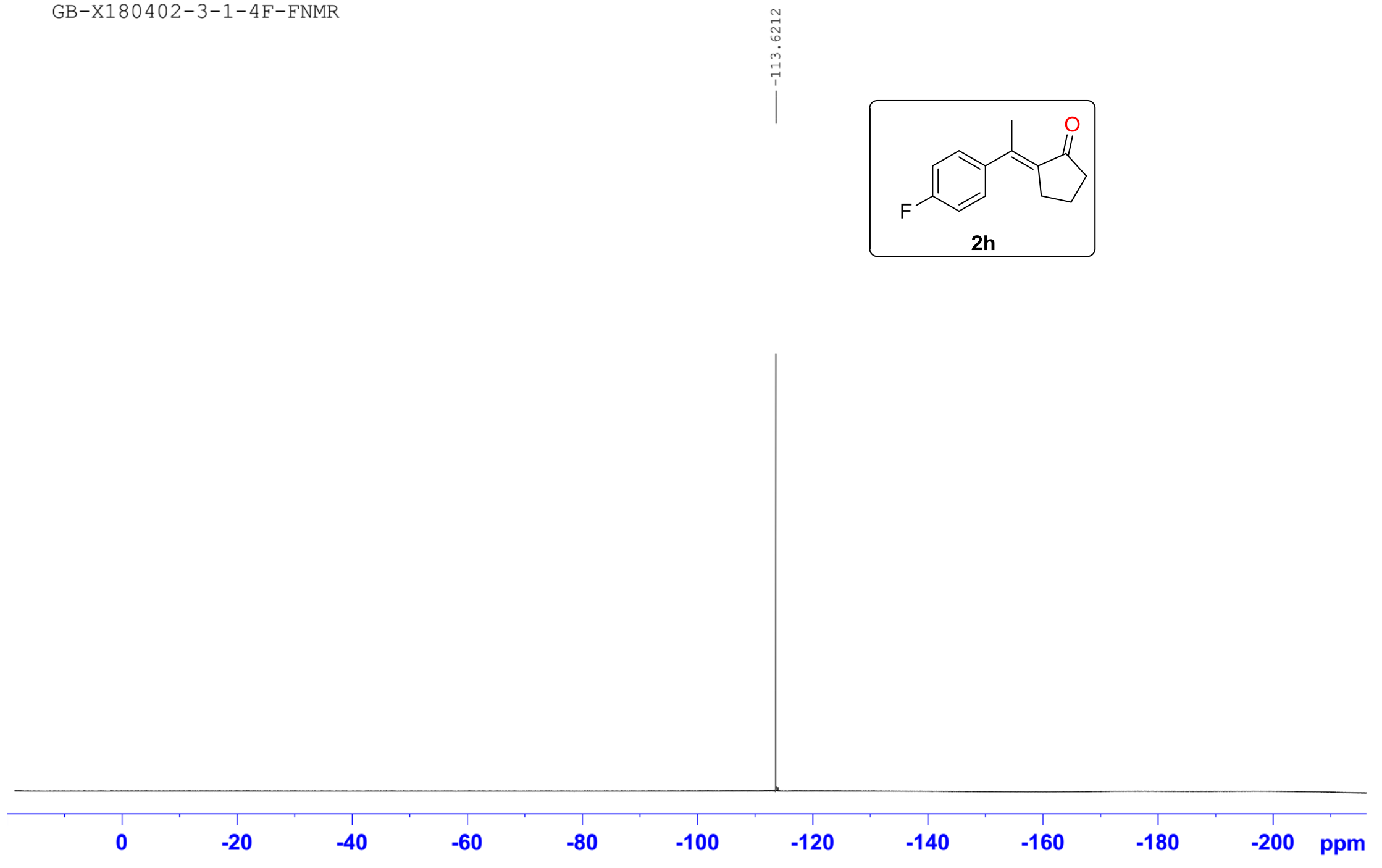
GB-X180129-1-7-4Cl-HNMR
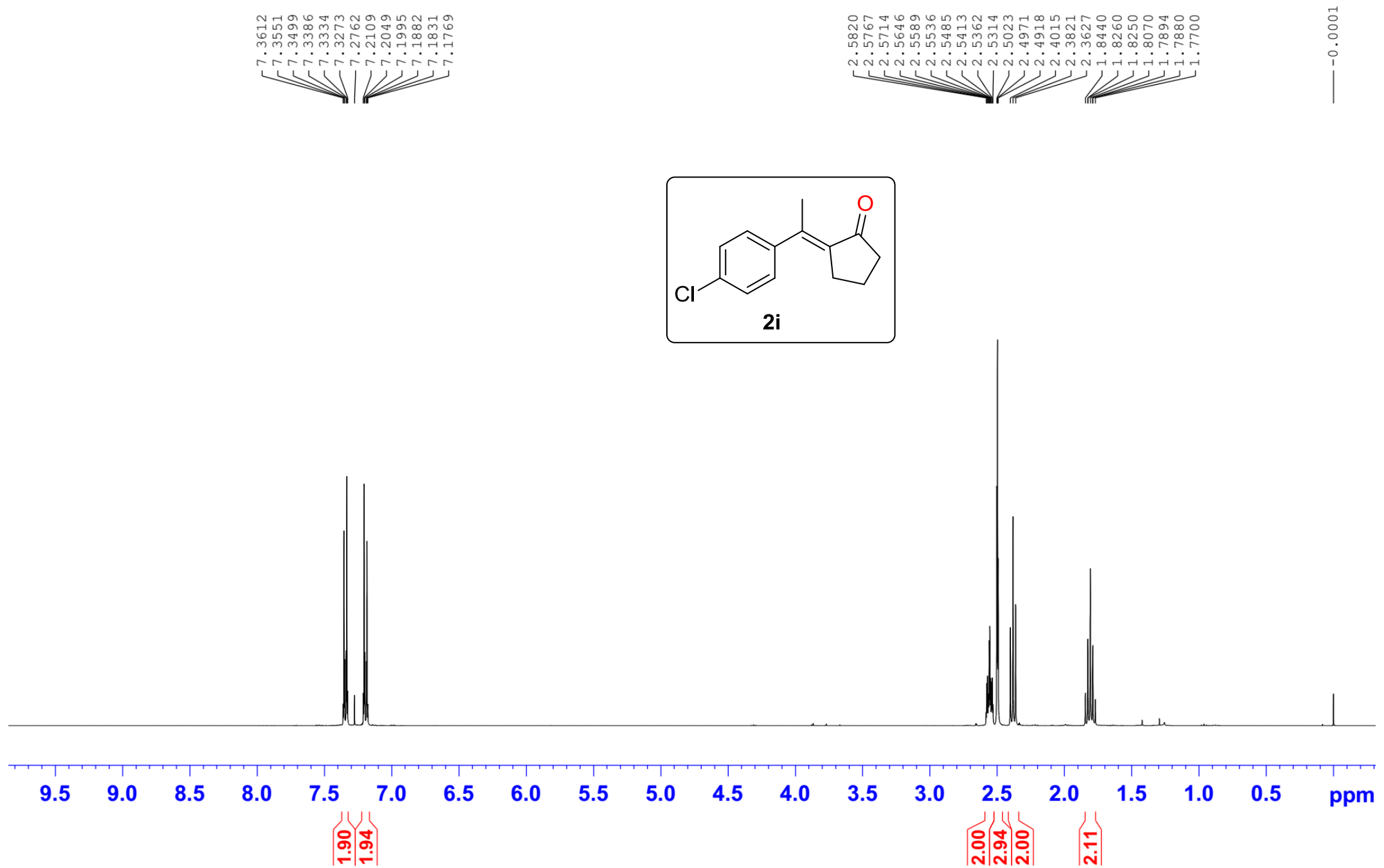
GB-X180129-1-7-4Cl-CNMR
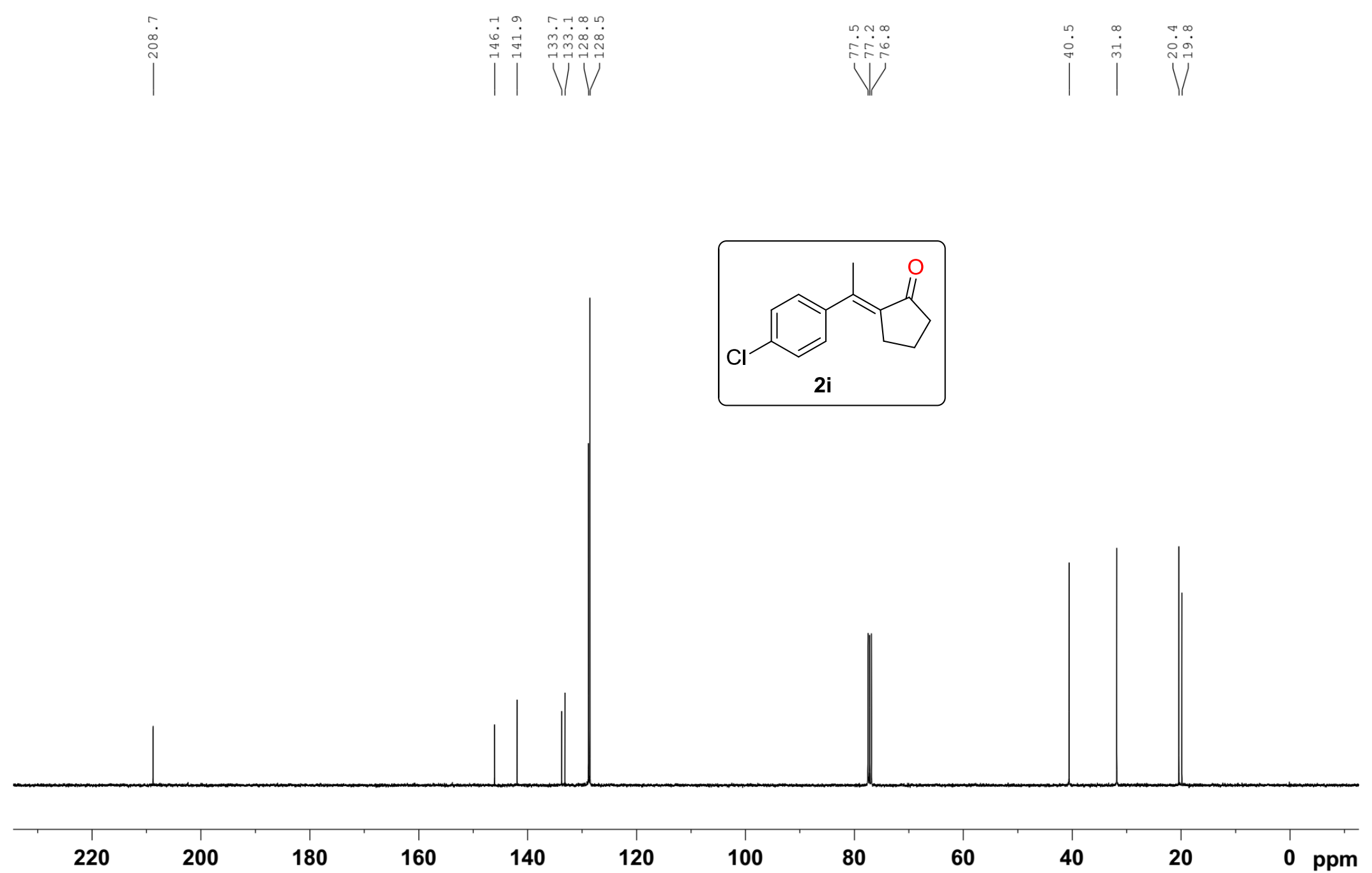
GB-X180614-4Br-HNMR
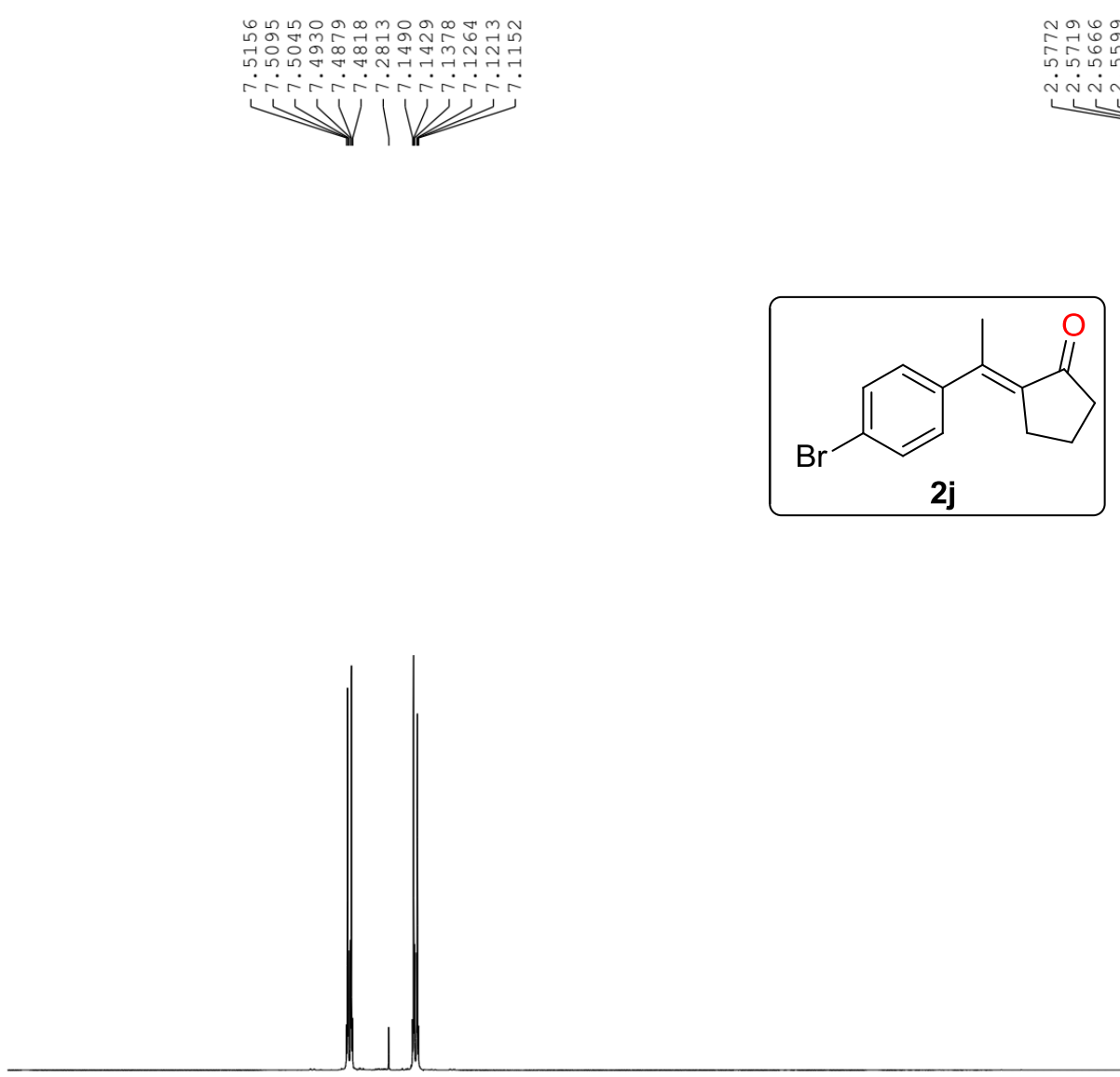
8
$\left|\begin{array}{ll} \\ \hdashline \\ \text { กิ }\end{array}\right|$

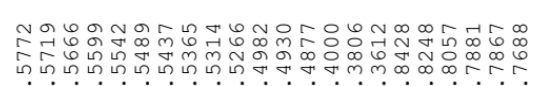

NNNNNNintin

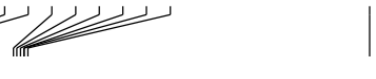

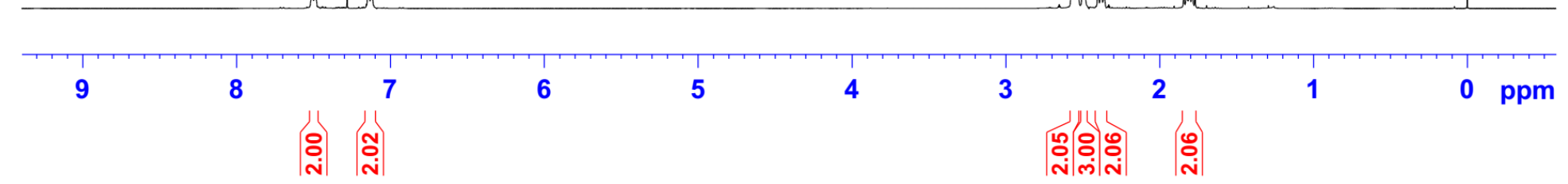


GB-X180614-4Br-CNMR

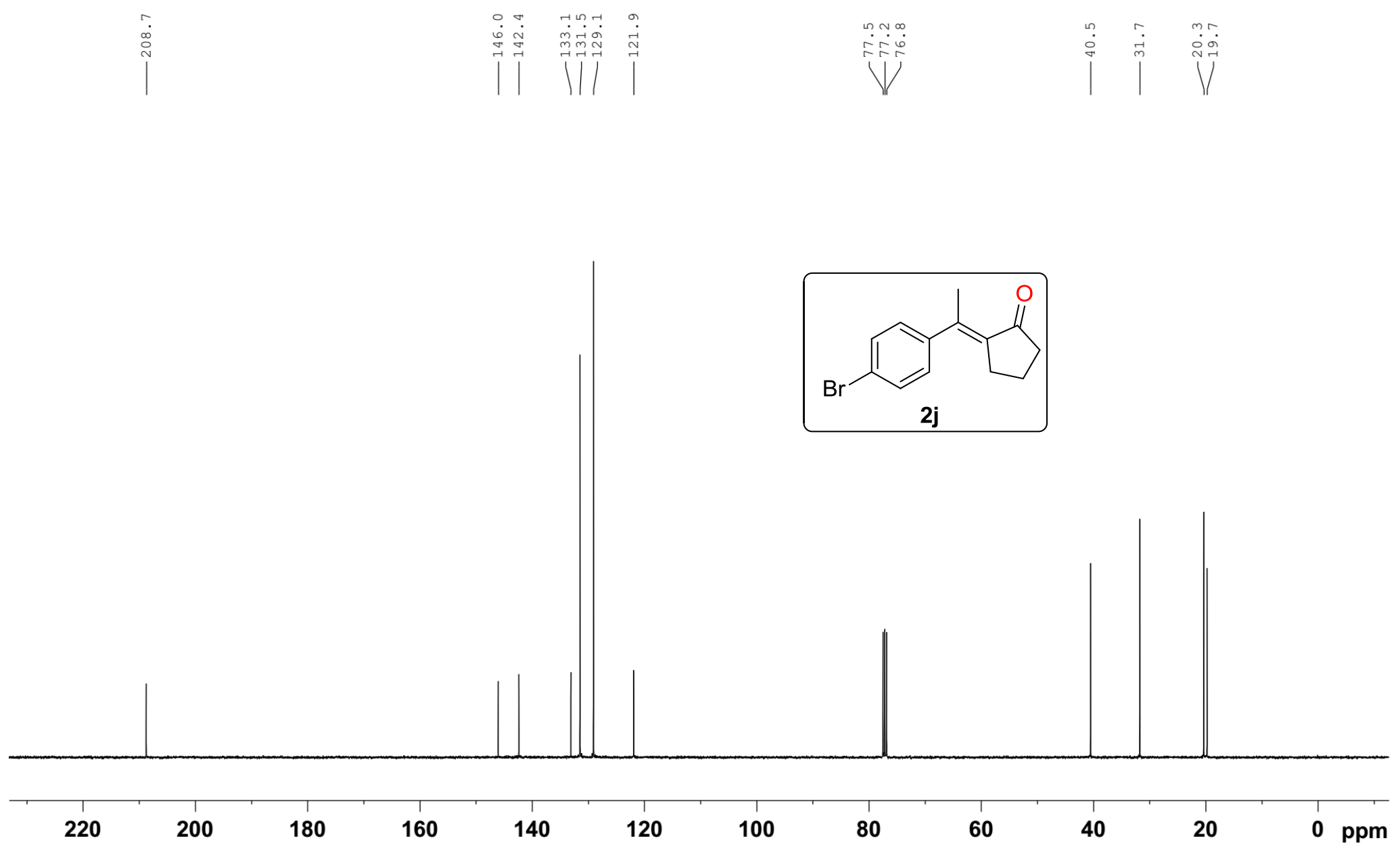


zSC-x180711-5-CDC13-HNMR

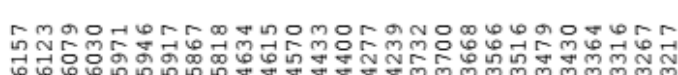

ririn

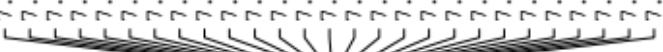

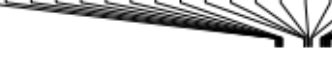
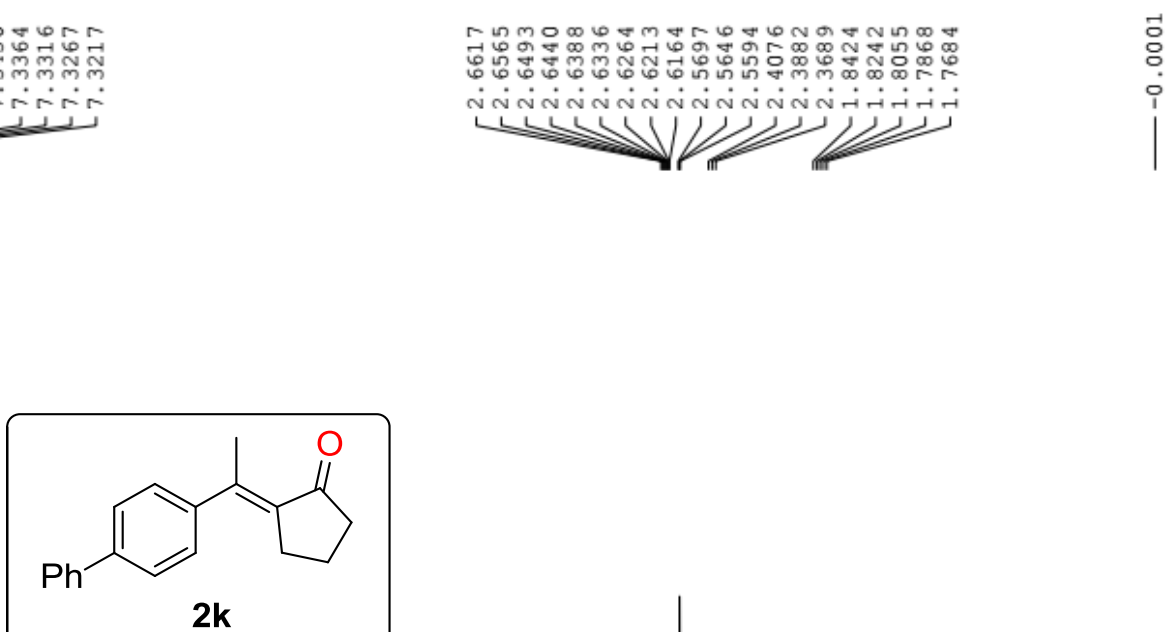

2k

$$
\text { (n) }
$$
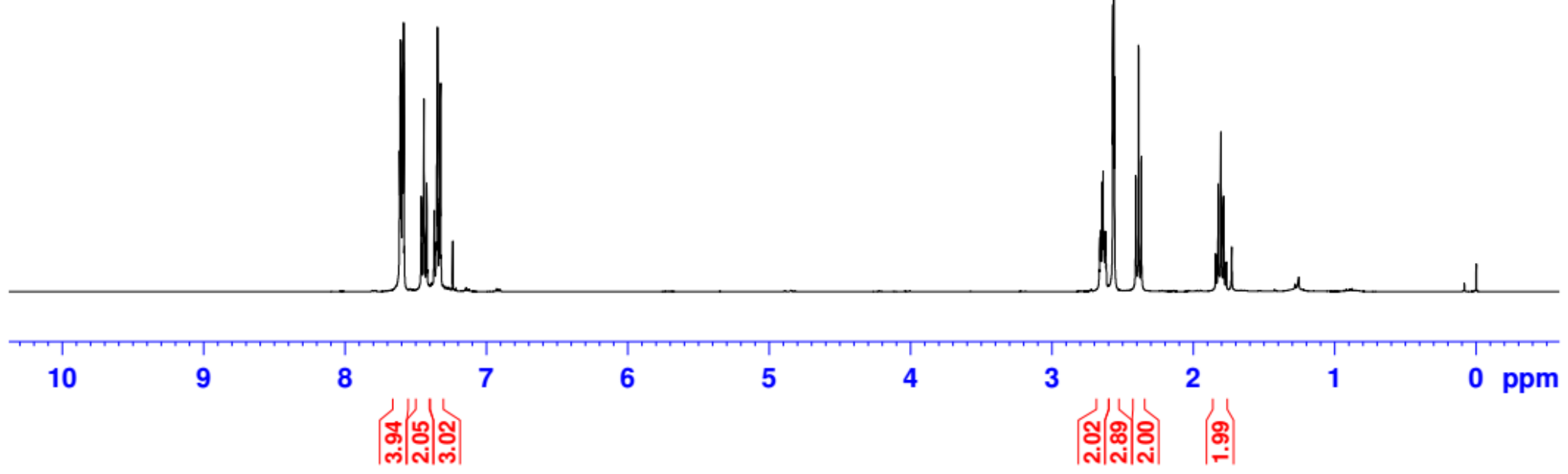

S97 
ZSC-X180711-1-5-CDC13-CNMR
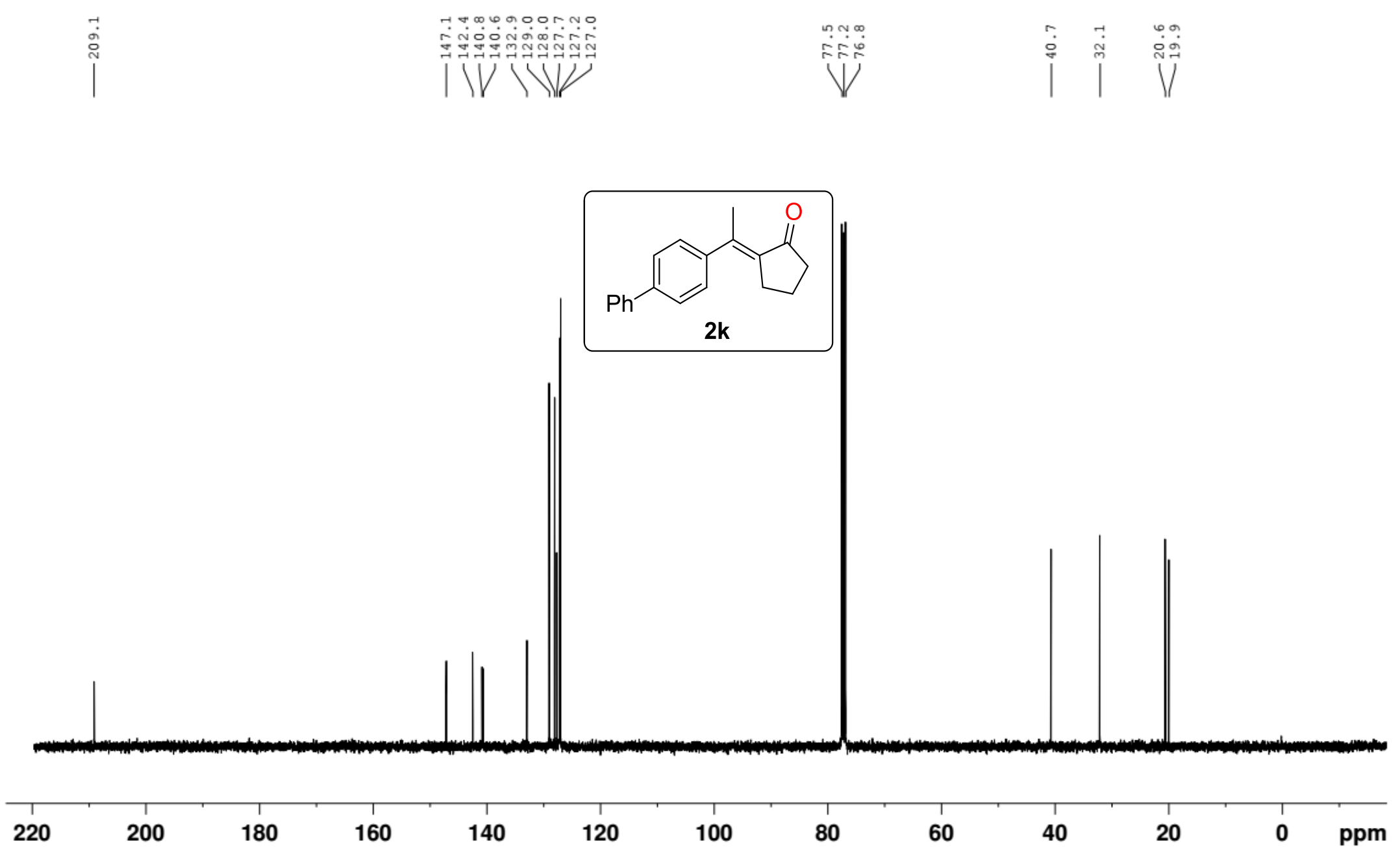
ZSC-X180714-1-CDC13-HNMR

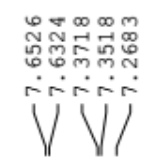

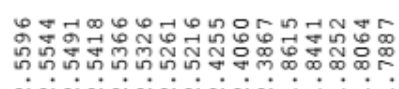

nNanNanN

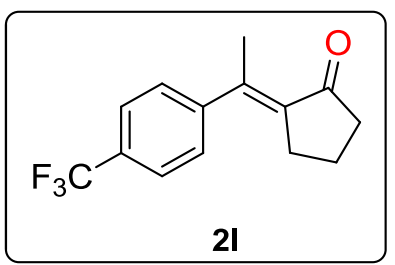

2 I

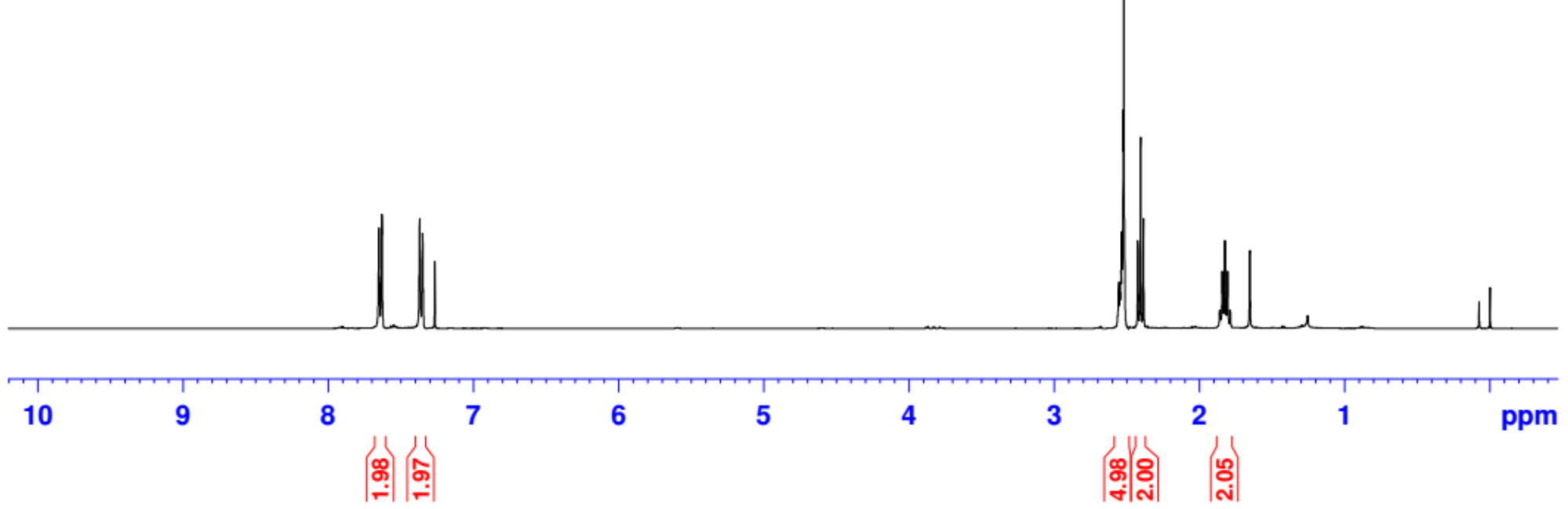



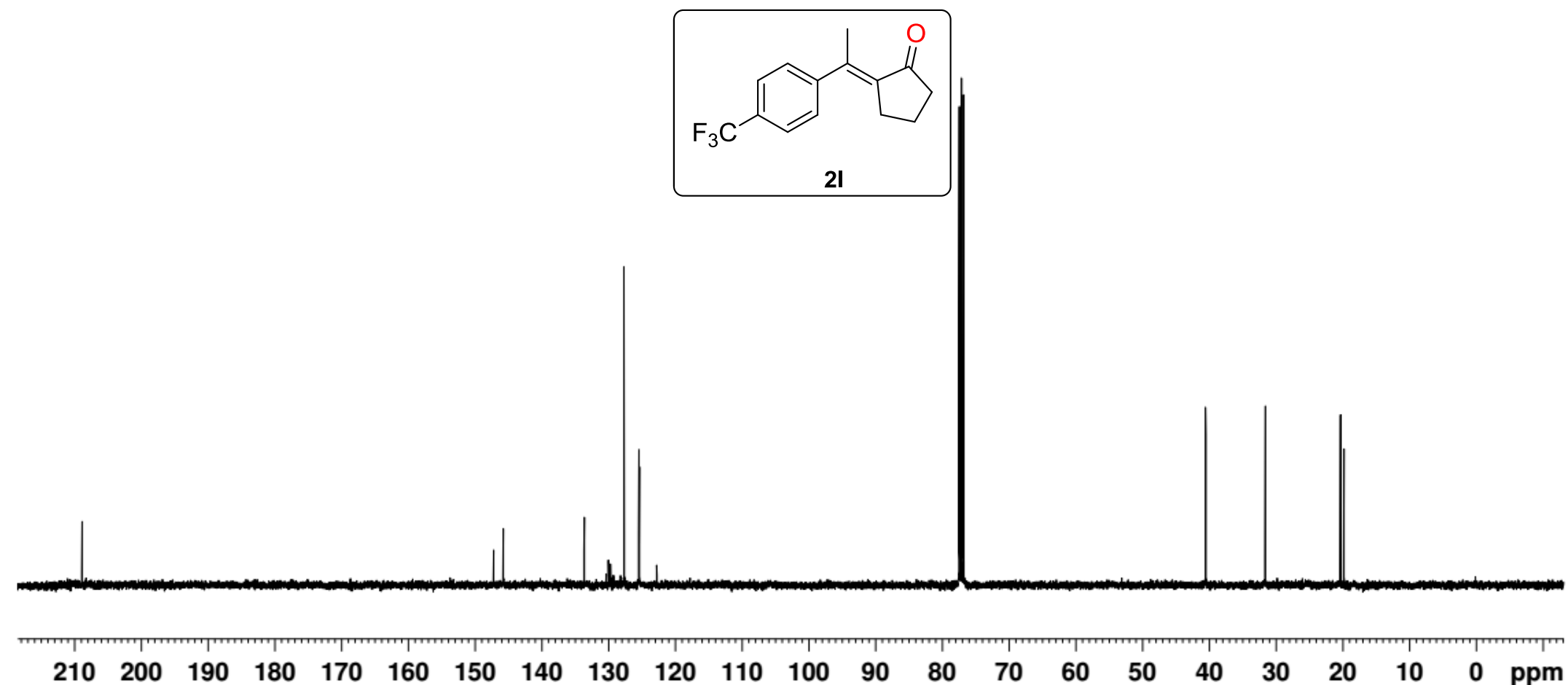
ZSC-X180714-1-CDC13-f-NMR

í
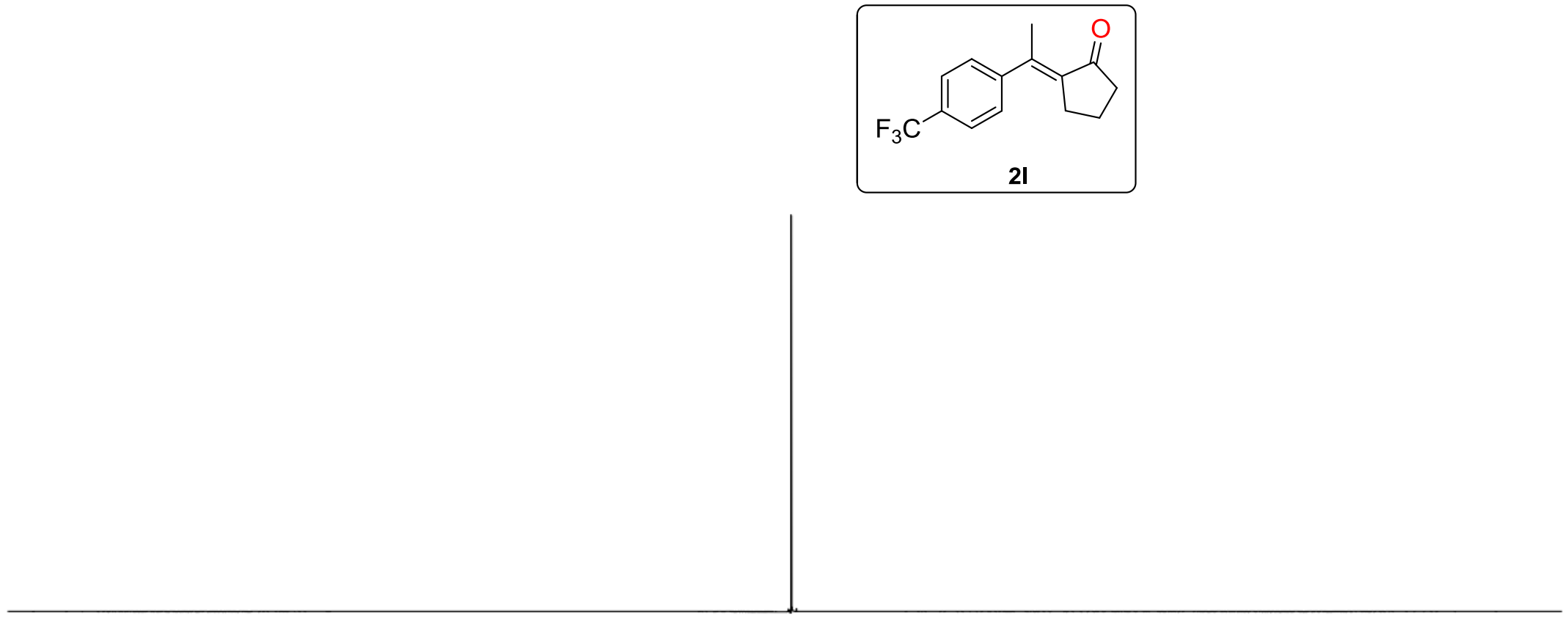

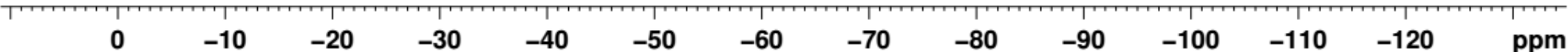


zsC-x180714-2-CDC13-HNMR

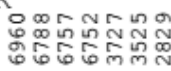

Vivivi

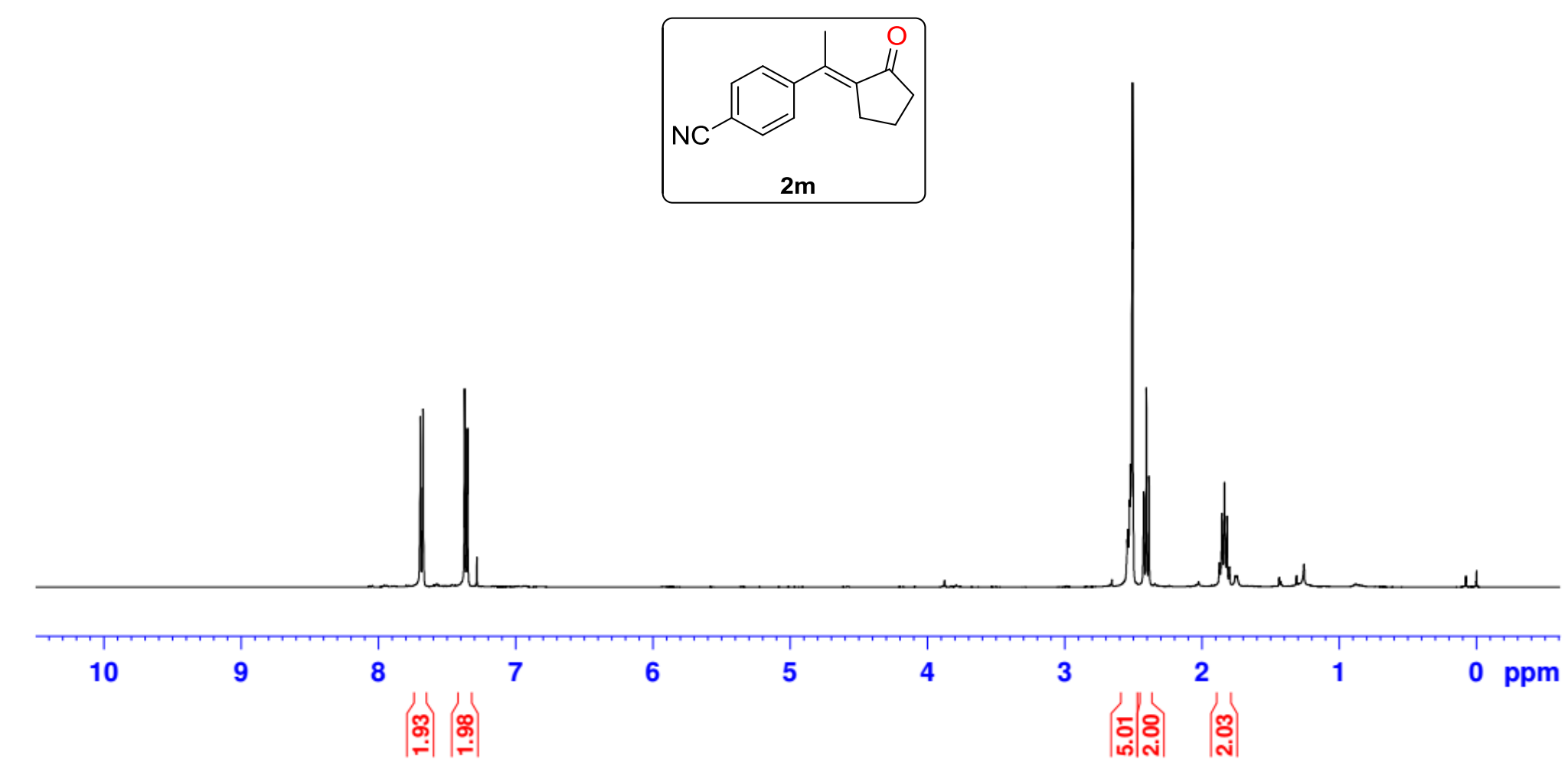

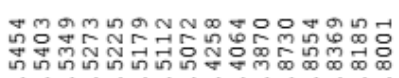

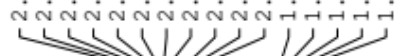


ZSC-X180714-2-CDC13-CNMR

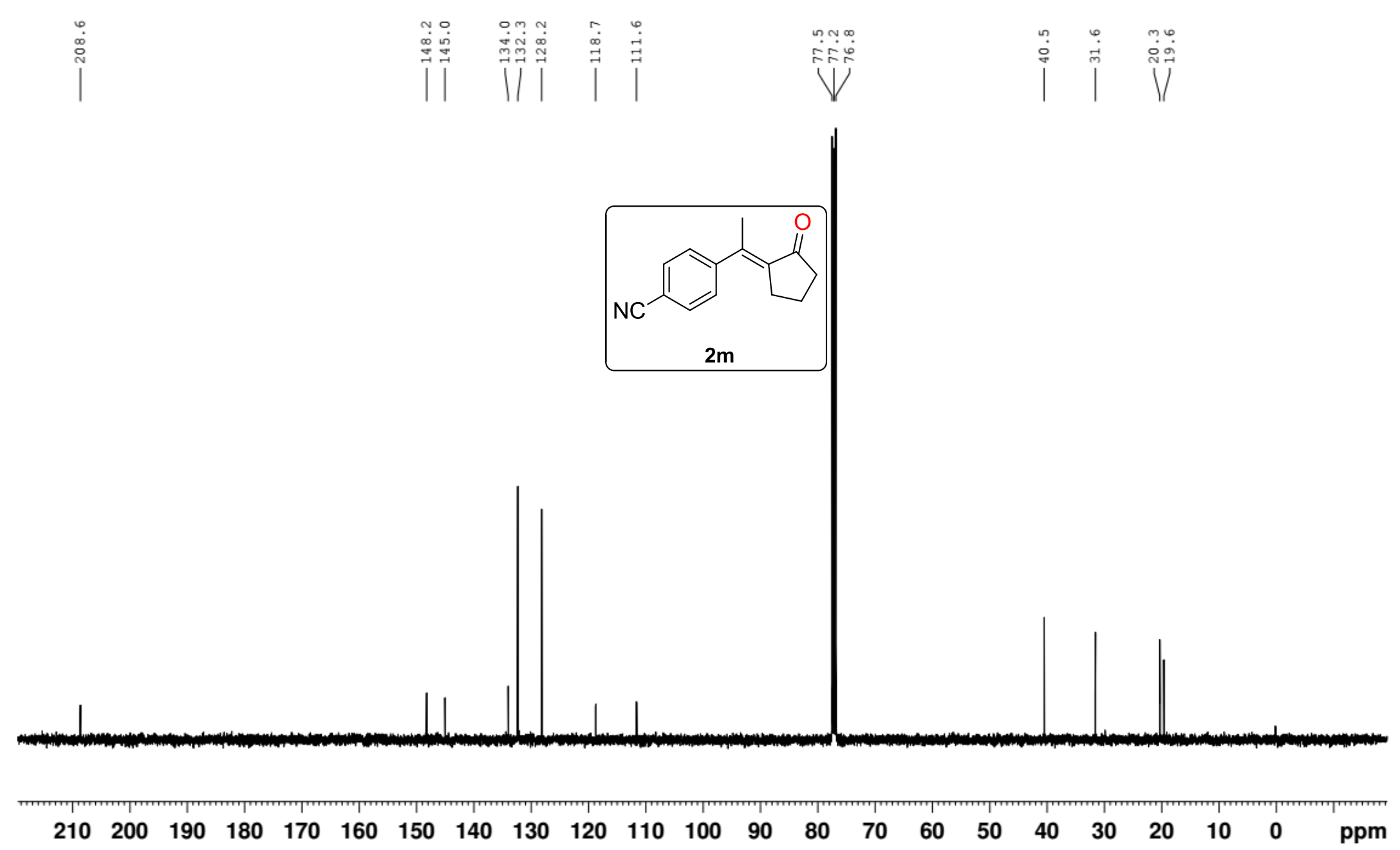



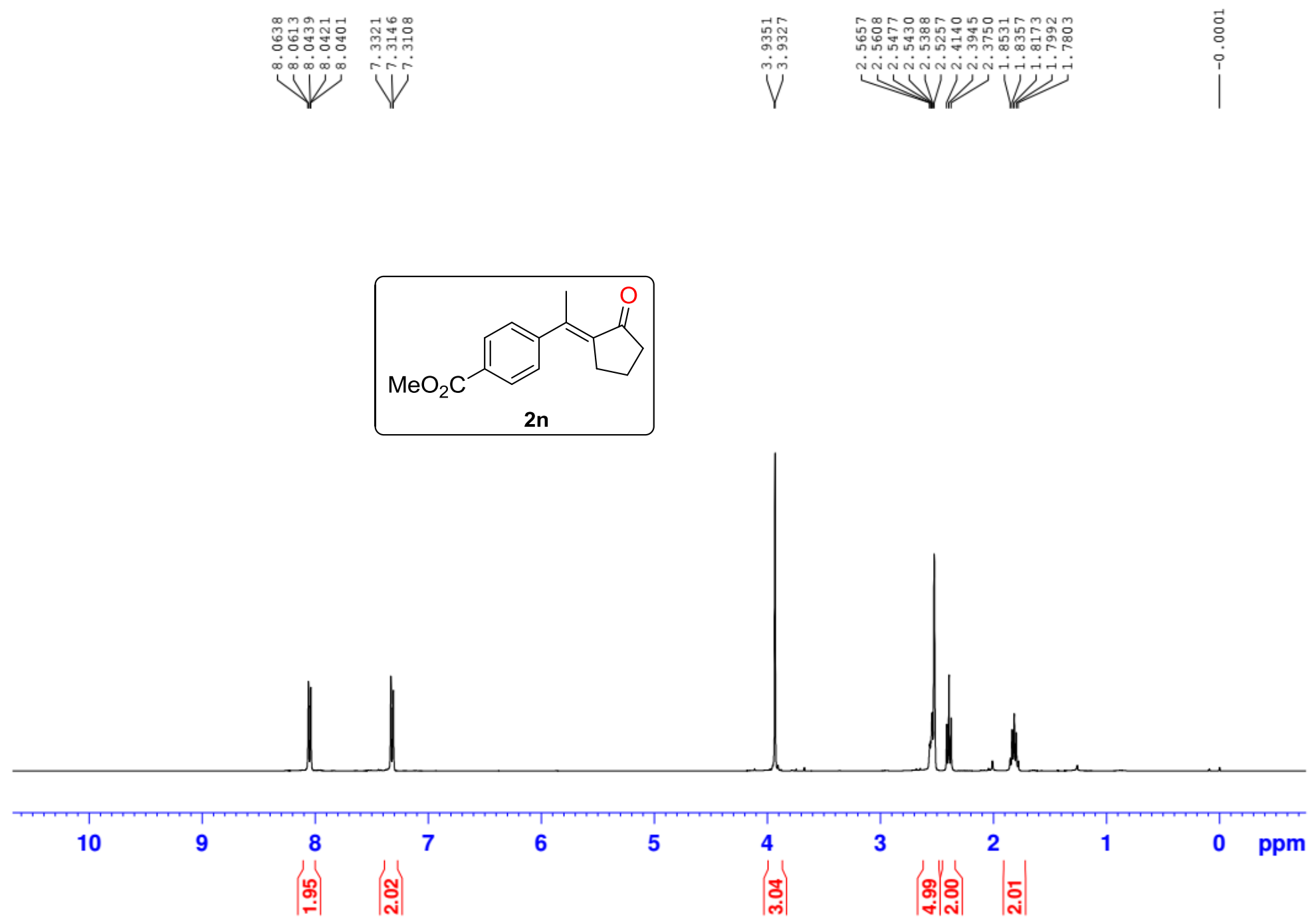
ZSC-X180714-6-CDC13-CNMR
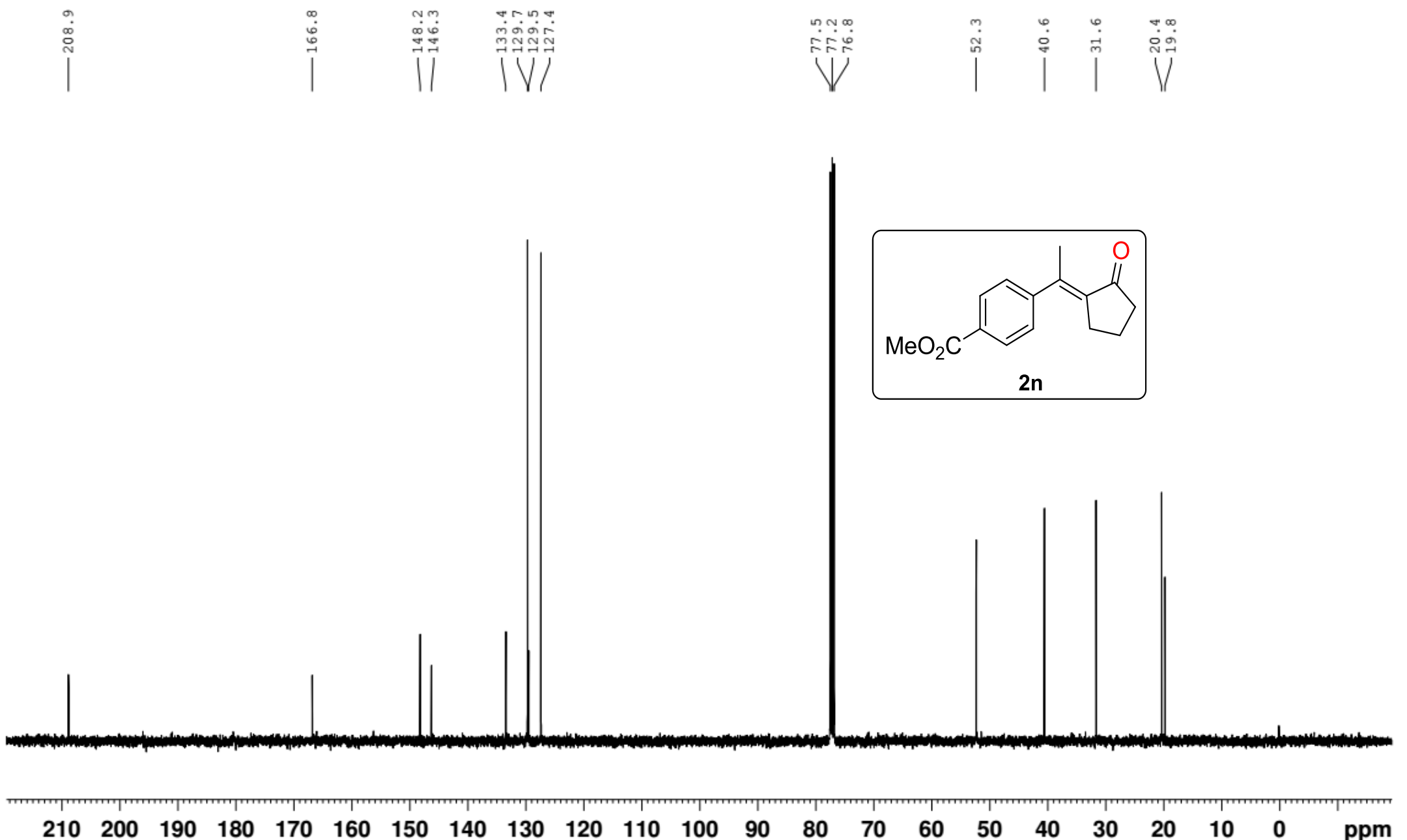
GB-X180308-1-1-2Na-HNMR
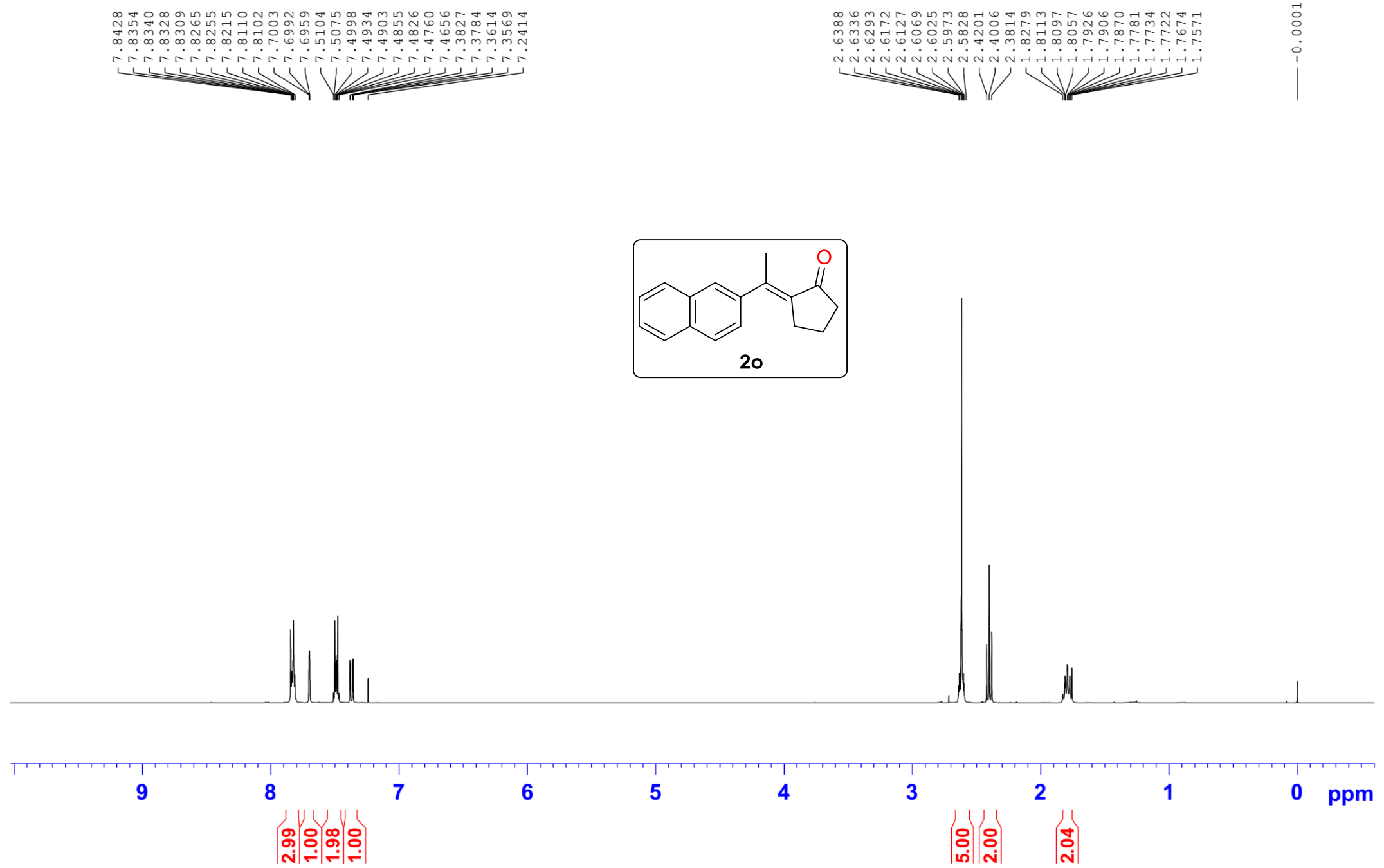
GB-X180308-1-1-2Na-CNMR
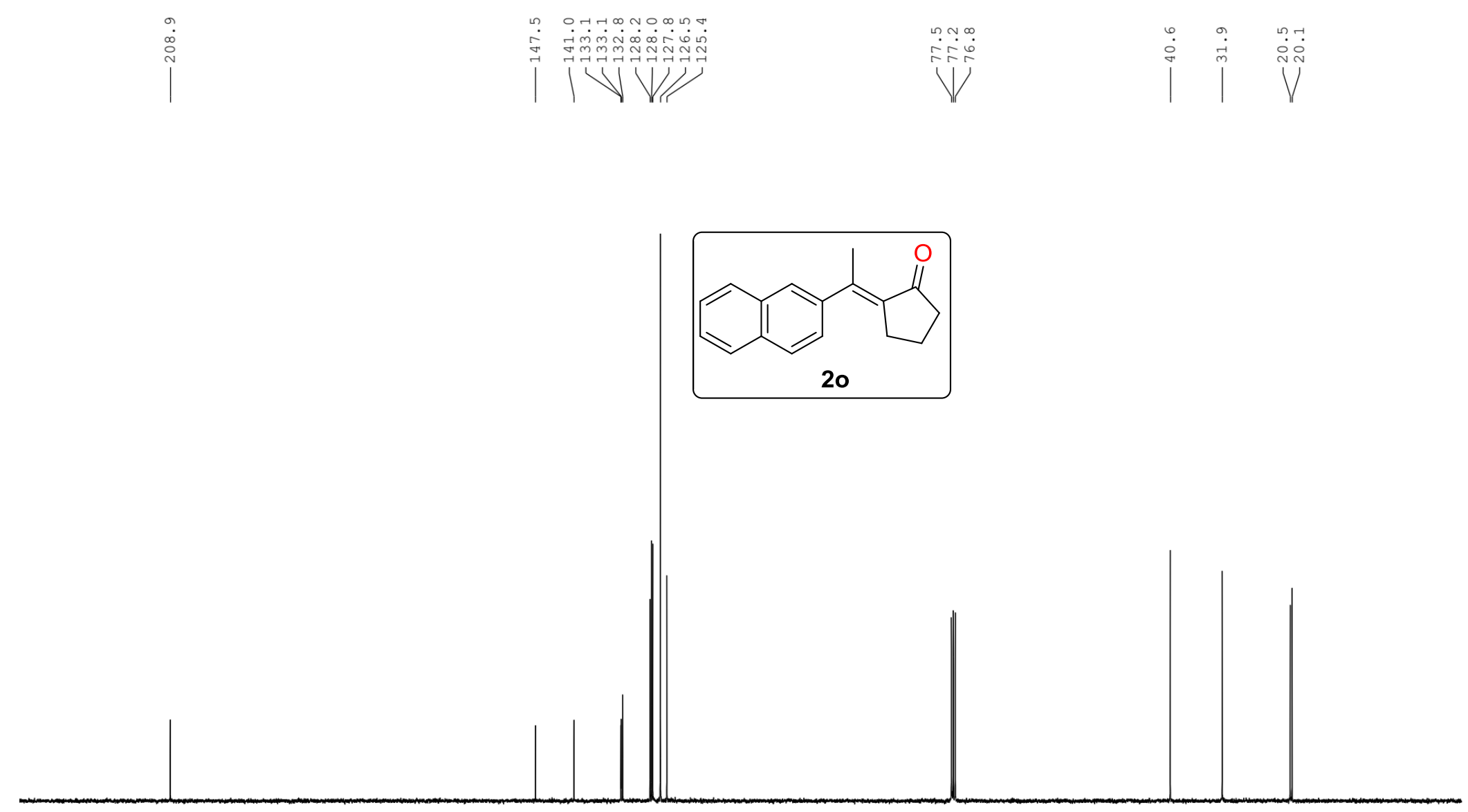

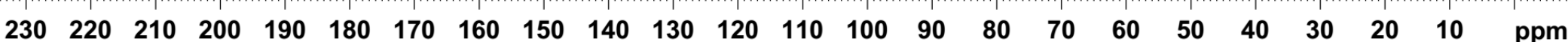


ZSC-X180717-2-CDC13-HNMR

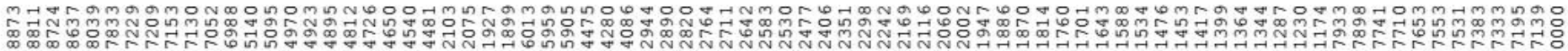

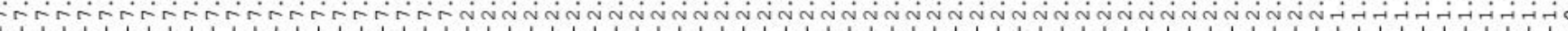
ind

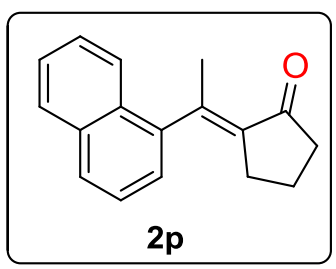

$2 p$

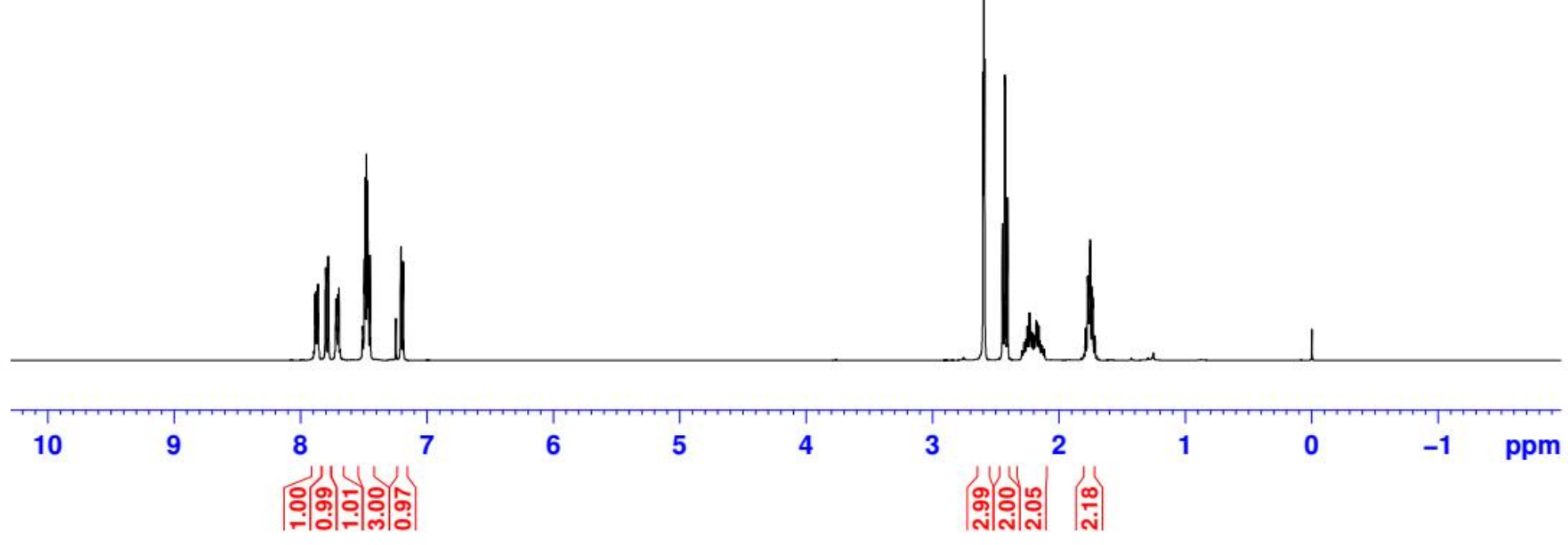


ZSC-X180717-2-CDC13-CNMR
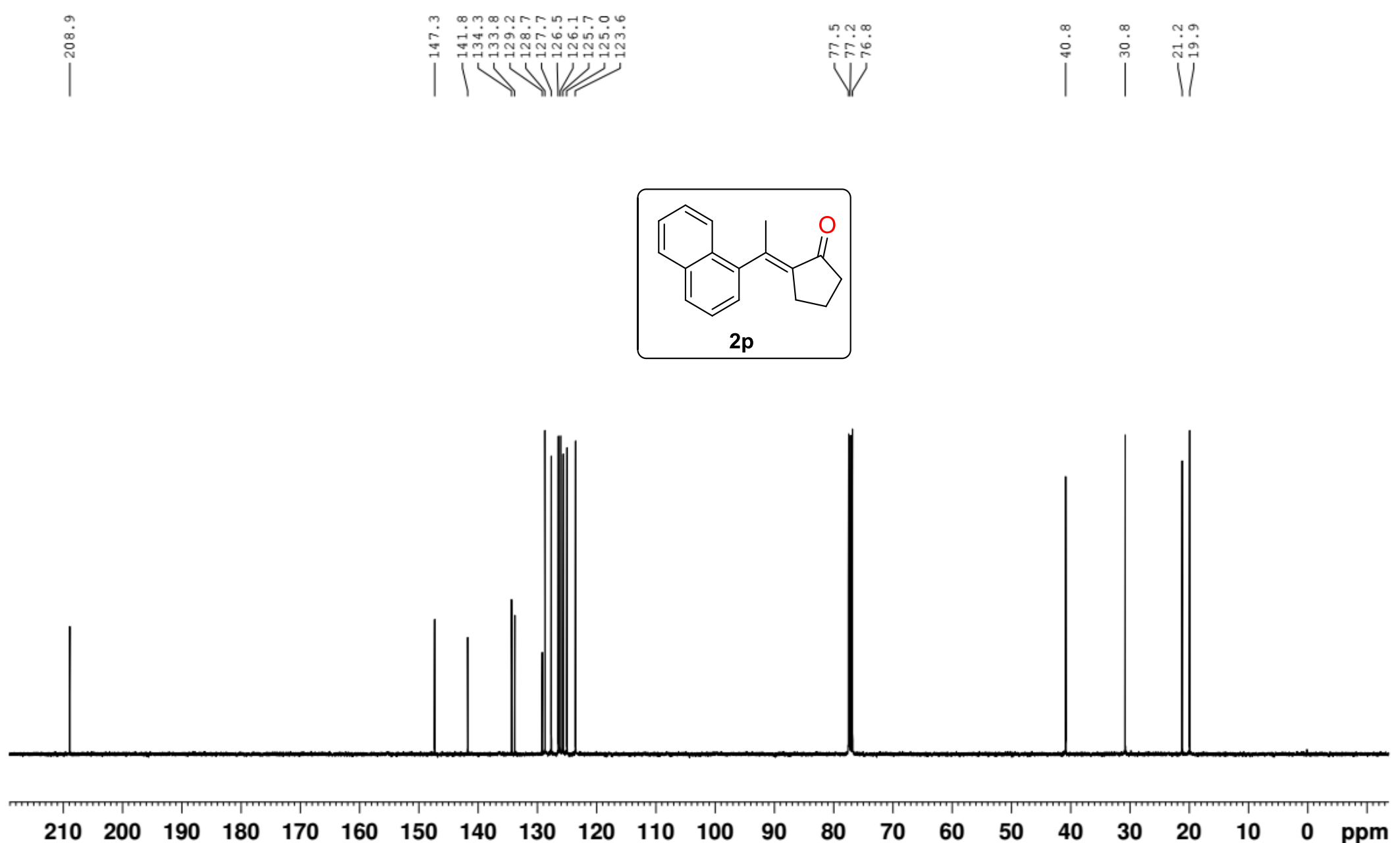
GB-X180614-1-2-MeMe-HNMR

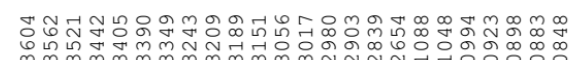

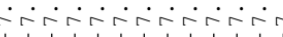

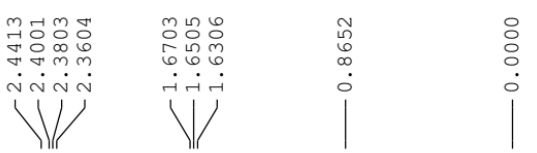

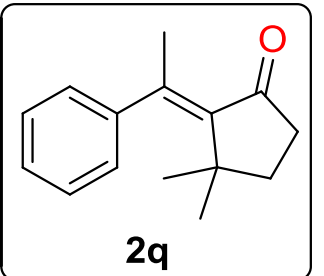

)

()

ลึ:

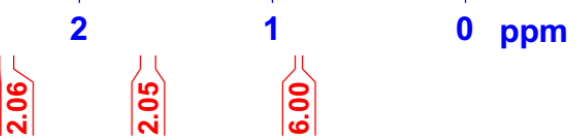


GB-X180614-1-2-MeMe-CNMR
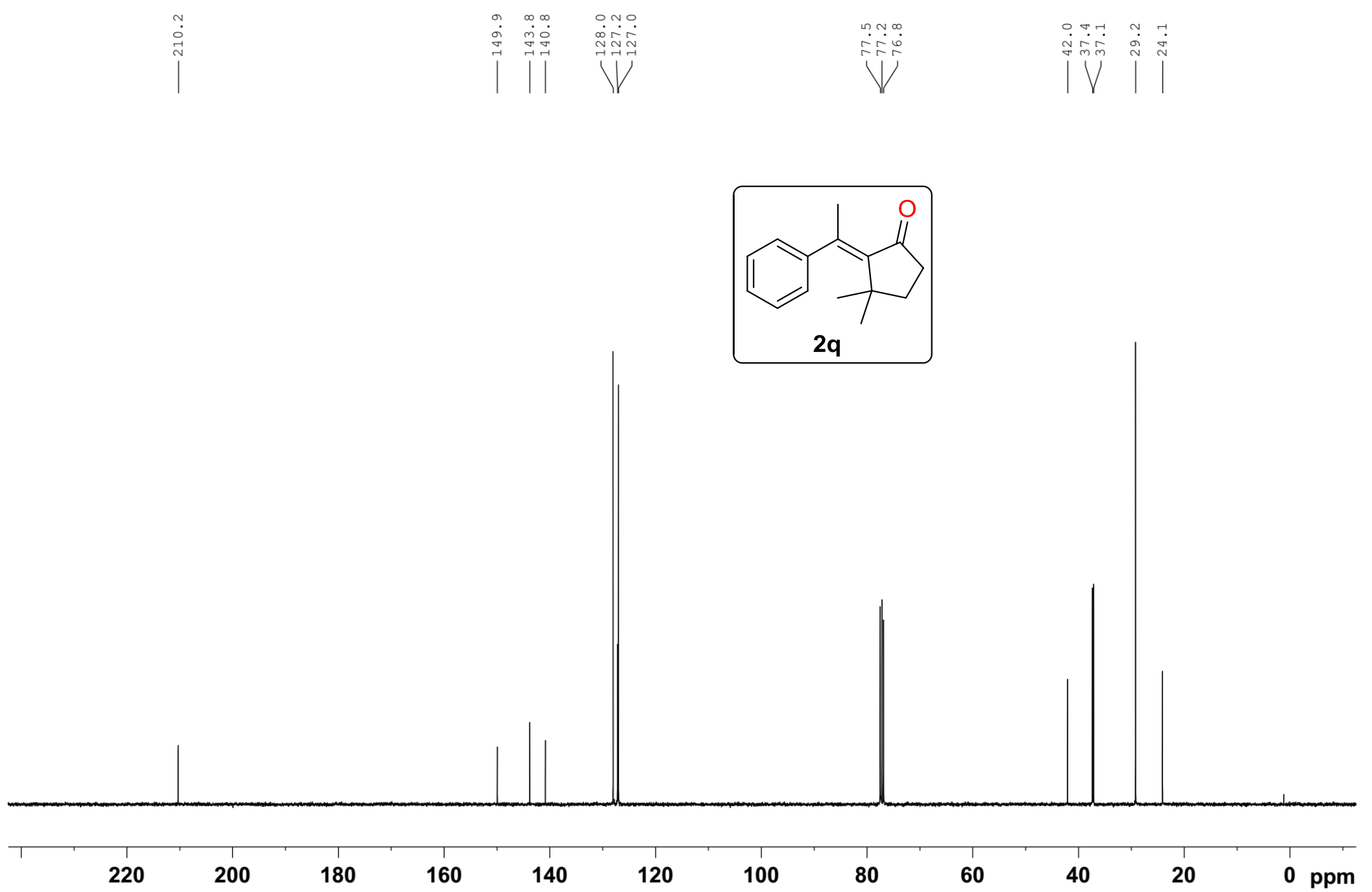


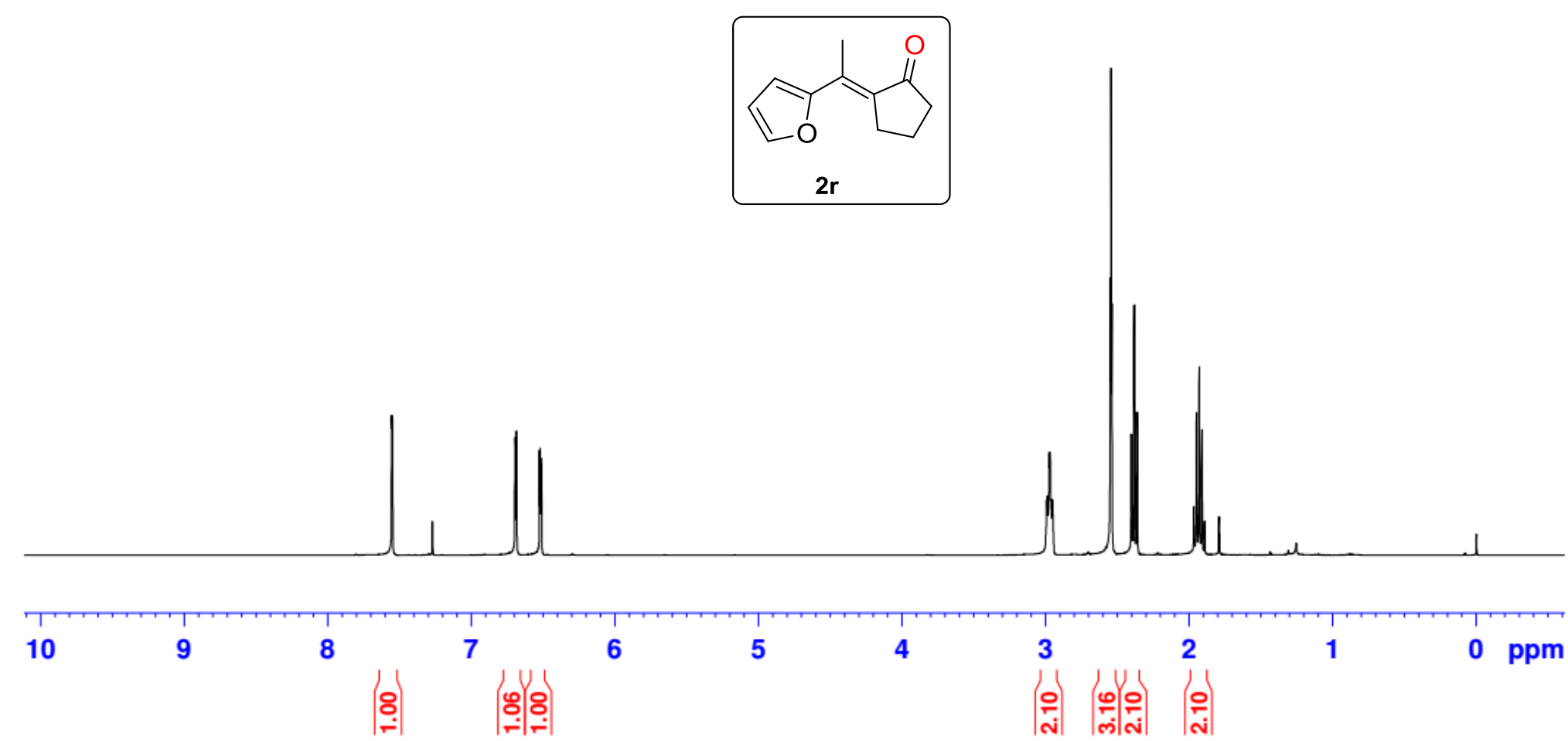


ZSC-X180905-2-CDC13-CNMR

|

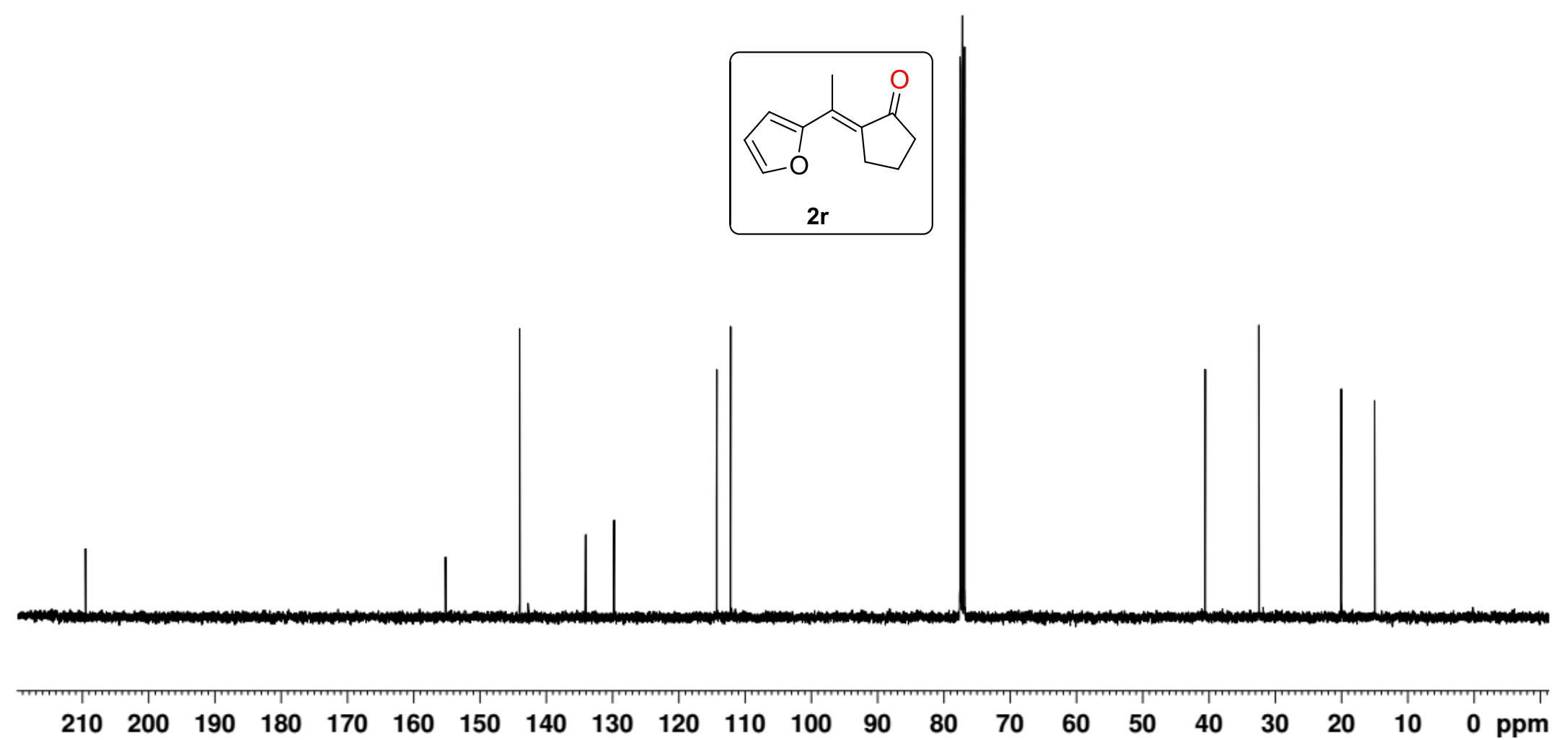


zsc-x180901-3-CDC13-HNMR

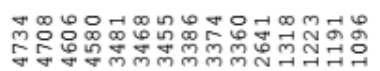

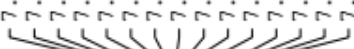

$\rightarrow$
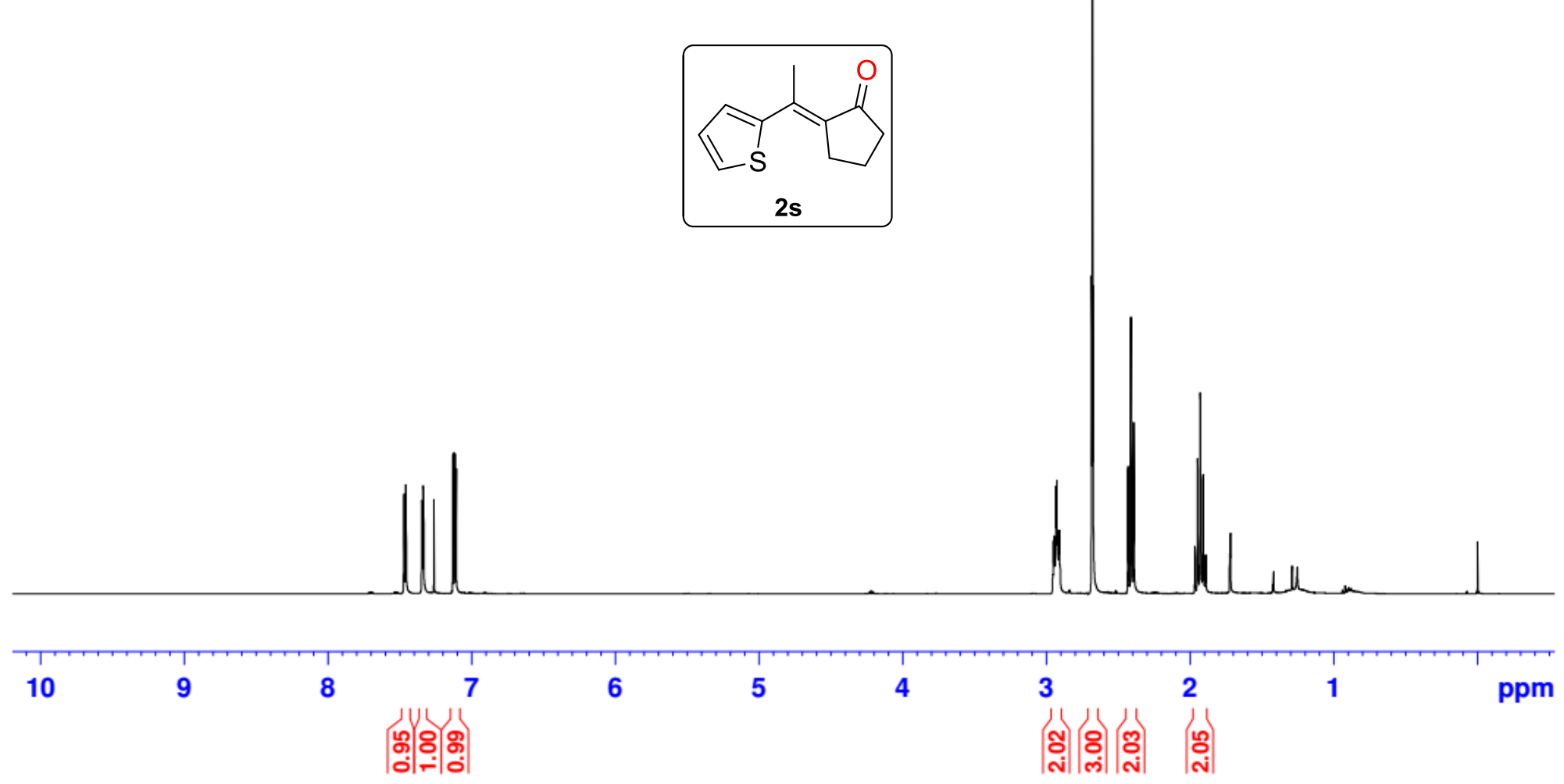
zsC-x180901-3-CDC13-CNMR
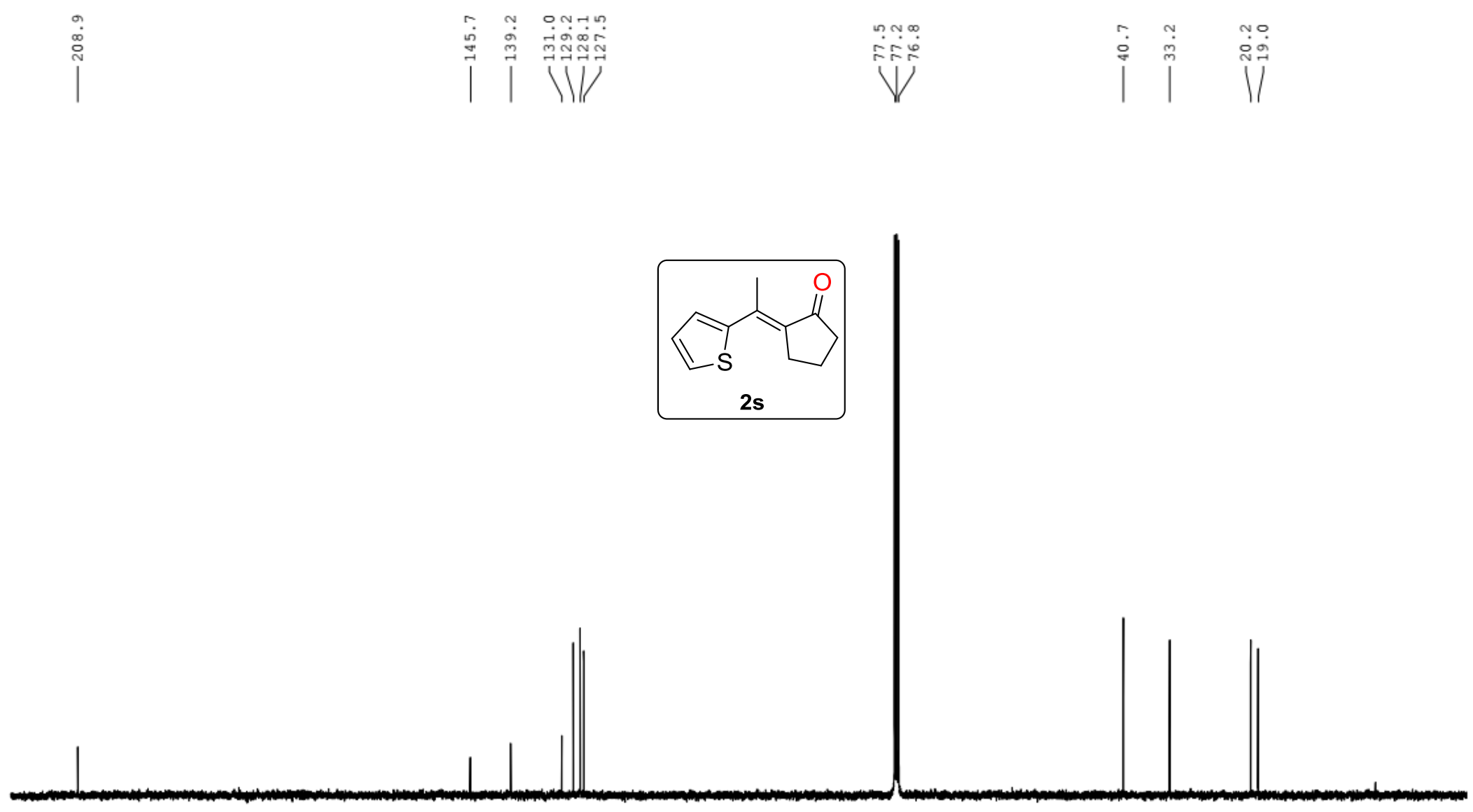

$\begin{array}{lllllllllllllllllllllllll}220 & 210 & 200 & 190 & 180 & 170 & 160 & 150 & 140 & 130 & 120 & 110 & 100 & 90 & 80 & 70 & 60 & 50 & 40 & 30 & 20 & 10 & 0 & \text { ppm }\end{array}$ 


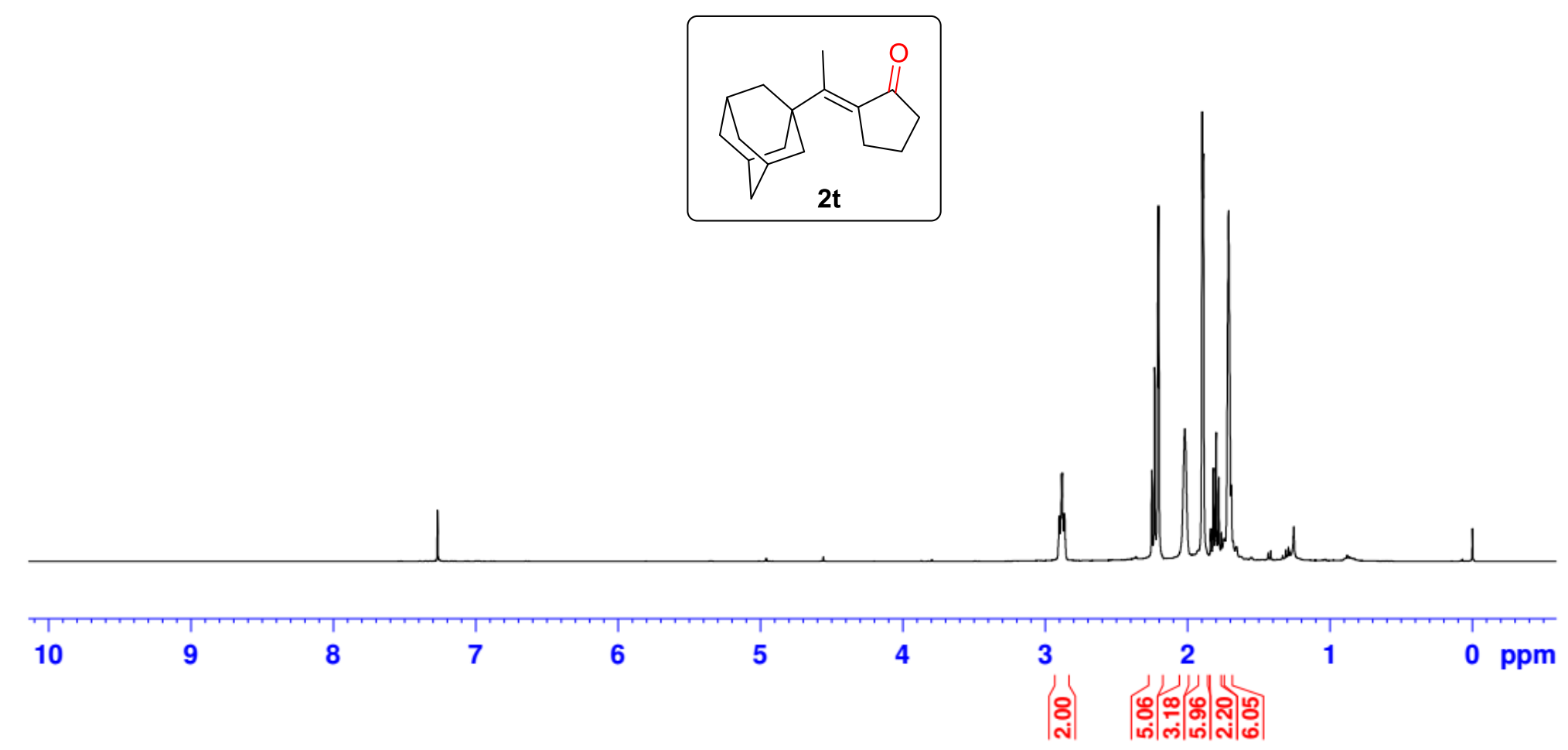


zsc-x18x12-3-CDC13-CNMR
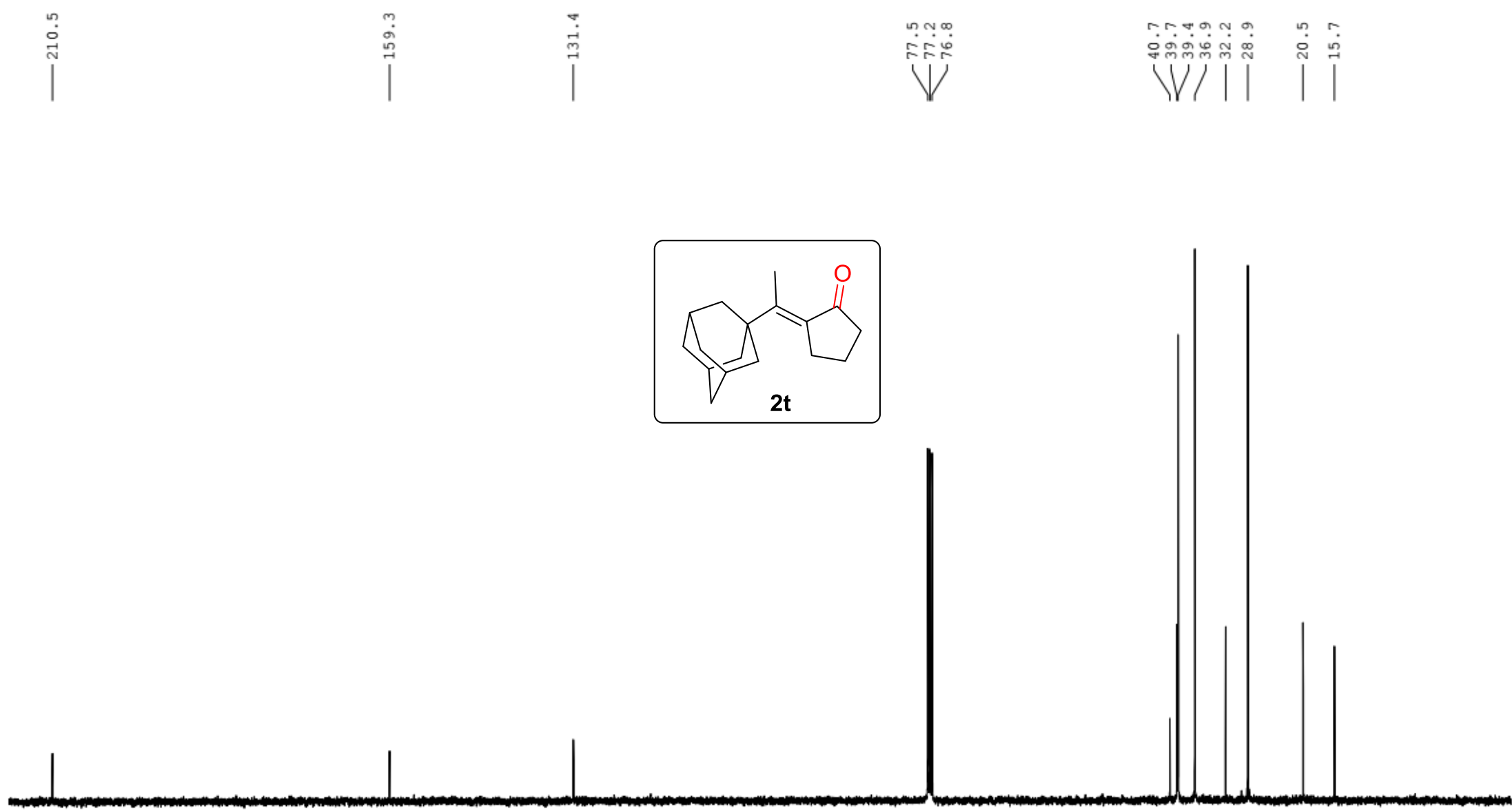

$\begin{array}{llllllllllllllllllllllll}210 & 200 & 190 & 180 & 170 & 160 & 150 & 140 & 130 & 120 & 110 & 100 & 90 & 80 & 70 & 60 & 50 & 40 & 30 & 20 & 10 & 0 & \text { ppm }\end{array}$ 
ZSC-X180813-1-CDC13-HNMR

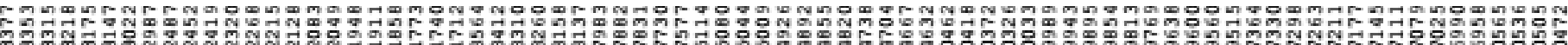

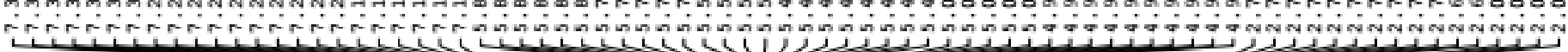

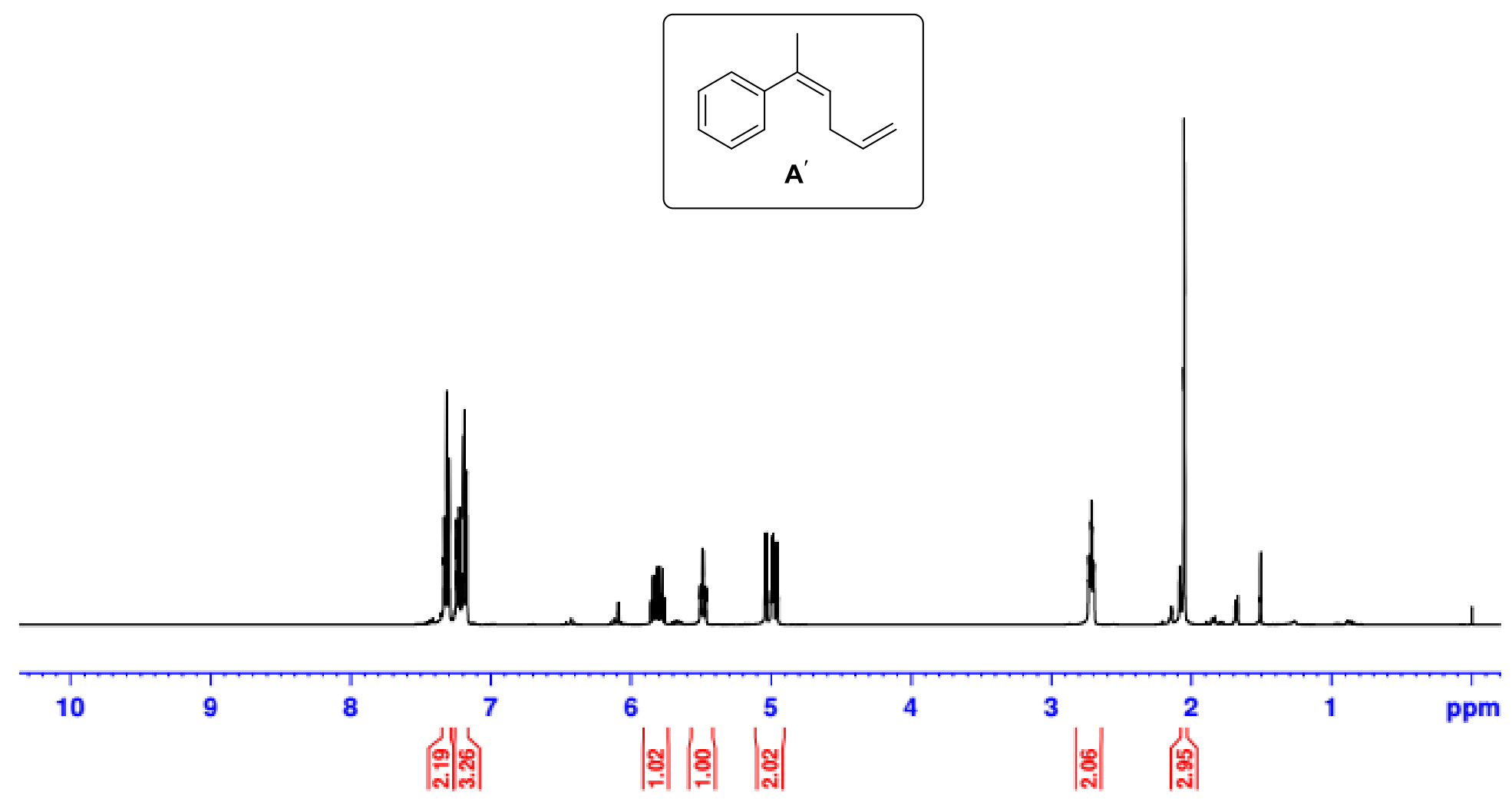



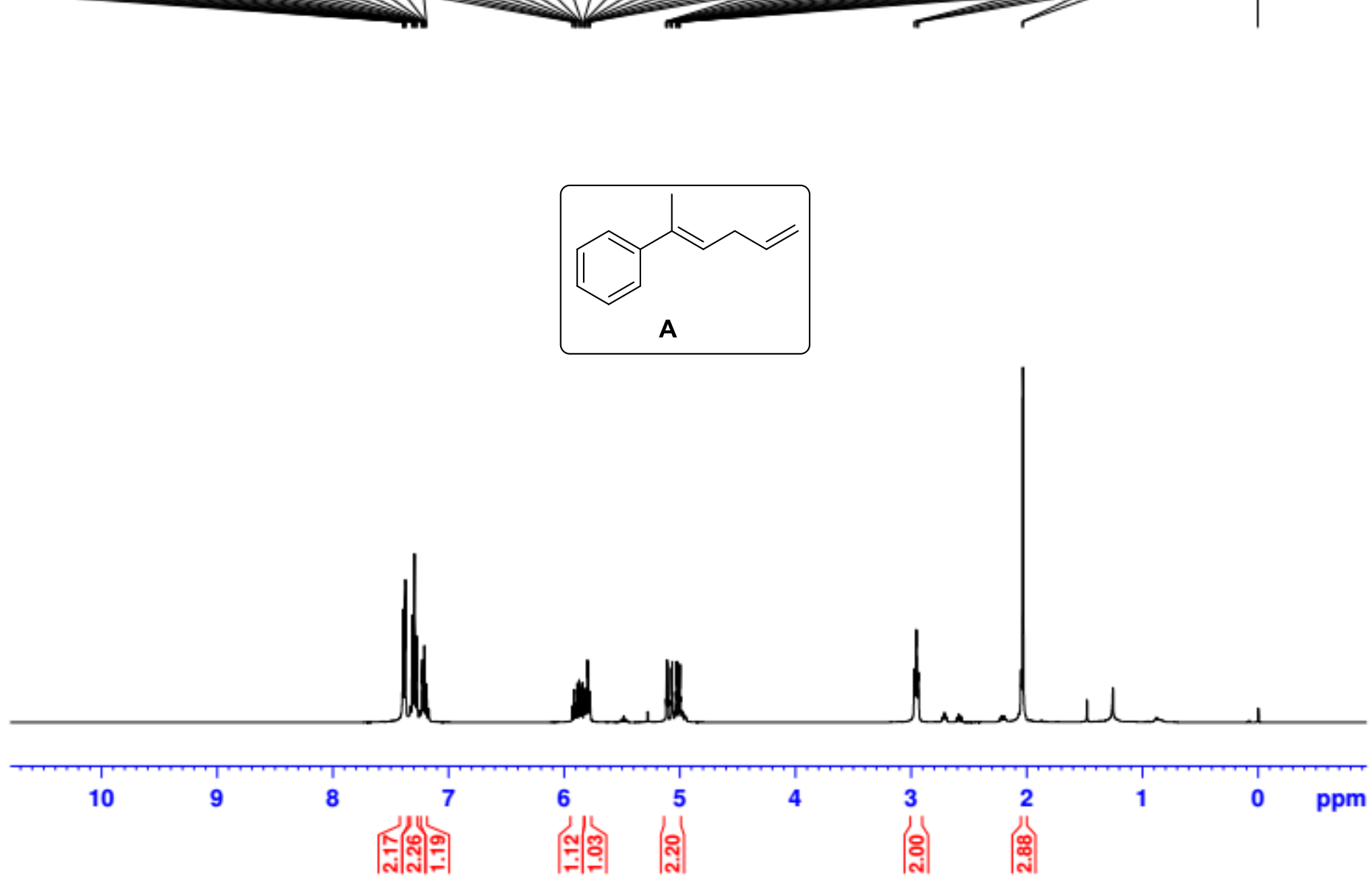